\title{
Laser Ablation-Inductively Coupled Plasma-Mass Spectrometer (LA-ICP-MS) in Geosciences: Further Improvement for Elemental Analysis
}

\author{
Dissertation \\ submitted to \\ the Mathematic-Scientific Faculties \\ of the Georg-August-University Göttingen \\ in partial fulfillment of the requirements \\ for the doctoral degree (Dr. rer. nat.) \\ according to the doctoral program \\ of the Georg-August University School of Science (GAUSS) \\ presented by
}

Shitou $\mathrm{Wu}$

From Hebei, China

Göttingen 2017 



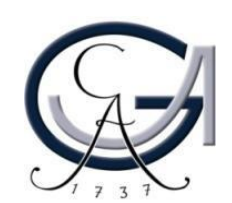

Thesis committee:

Supervisor: Prof. Dr. Gerhard Wörner (GZG, Department Geochemistry)

Co-supervisor: Dr. Klaus Simon (GZG, Dep. Geochemistry)

\section{Examination committee:}

Prof. Dr. Gerhard Wörner (GZG, Dep. Geochemistry)

Prof. Dr. Matthias Willbold (GZG, Dep. Isotope Geology)

Prof. Dr. Sharon Webb (GZG, Dep. Mineralogy)

Prof. Dr. Andreas Pack (GZG, Dep. Isotope Geology)

Dr. Klaus Simon (GZG, Dep. Geochemistry)

Dr. Ingo Horn (Institut für Mineralogie, Leibniz Universität Hannover)

Day of defense:

24. August 



\section{Acknowledgments}

A doctoral thesis is always the outcome of the constructive interaction of various persons with both scientific and non-scientific background, whose contributions shall be acknowledged at this point.

Firstly, I would like to thank my supervisor Gerhard Wörner, who provides me the Ph.D. position at Göttingen University. He gave me such freedom so that I could follow my own interests and carried out the thesis project. He supported me to participate in five international conferences, which really open my eyes to the world research about LA-ICP-MS technique, and this lets me touch the cutting edge topics.

Then, I thank for the scholarship funding from China Scholarship Committee (CSC). Without this financial support, I could not continue my Ph.D. project in Germany.

Klaus Simon, my co-supervisor, is greatly thanked here for the four-year supervision in LA-ICP-MS laboratory. He provided me free-charged measurement time; so that I could learn the instrument in practice, which is very crucial for the beginner. He always encourages me to continue when I am frustrated with the unexpected results. Without his generous help, especially his 20-year working experience in LA-ICP-MS, I could not master the instrument in such short period and could not finish this thesis.

Burkhard C Schmidt, Volker Karius, István Dunkl and Yiling Xiao are thanked here for the supervising laboratory work, the helping with data interpretation as well as the fresh idea about my projects. I learned how to produce homogeneous glass from powdered rock sample from Burkhard C Schmidt, how to operate the wet-milling machine from Volker Karius, the zircon dating experience from István Dunkl. Yilin Xiao provided me the samples for the ablation rates project. I convey my sincere gratitude to them at this point. 
The thesis project would not have been possible without the help and reliable support of my colleagues: Andreas Kronz, Gerald Hartmann, Raffaella Silvia Iovine, Smruti Sourav Rout. Andreas Kronz is specially thanked for his generous helpfulness in the EMPA lab. Gerald Hartmann is thanked for the supervision of tableting pellet presser. Raffaella Silvia Iovine and Smruti Sourav Rout are greatly appreciated for the indispensable helps during the Ph.D. period.

Here, I also thank Yaping Wang, Chunxue Xu and other group members from the Institute of National Research Center of Geoanalysis. They provided me several powdered reference materials for free and helped with the manuscript writing. They also offered me a small funded project for the development of $N E W$ glass reference materials for LA-ICP-MS.

Finally, I would like to thank my family members, especially my wife, Xiyang Liu. Without her trust and supports, I cannot finish the thesis in a quite smooth way. My sister and brother are thanked to here for the past several years taking care of my mom, grandmother. Without them kind support, I could not stay in Germany for four years feeling at ease. 


\section{Contents}

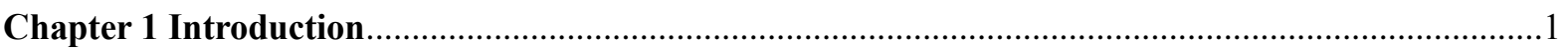

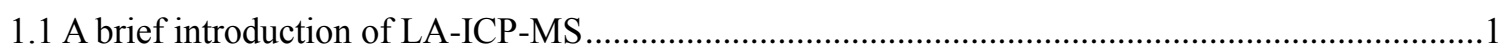

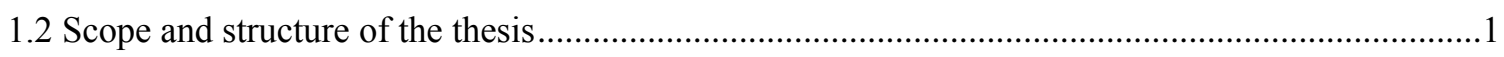

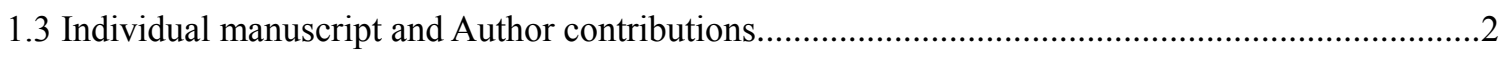

Chapter 2 Quantification of LA-ICP-MS transient signals based on Iolite software package ..............6

Chapter 2.1 LA-ICP-MS Transient Signal Quantification of NIST, MPI-DING, USGS and CGSG

Glass Reference Materials by Ratioing, Standardization, and Normalization (RSN)...........................6

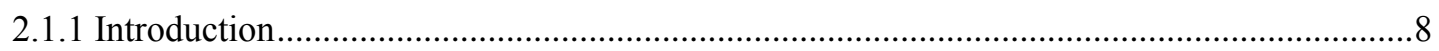

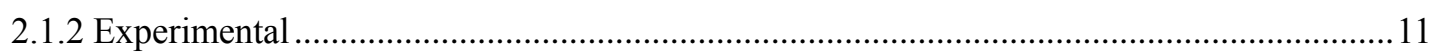

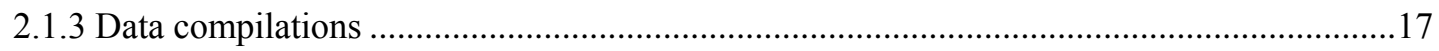

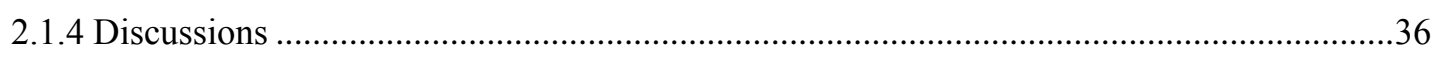

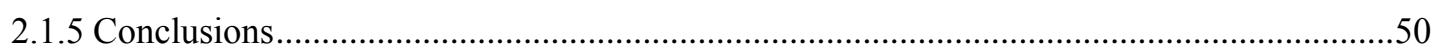

Chapter 2.2 Laser Ablation Inductively Coupled Plasma Mass Spectrometer: A quantification Strategy

Based on Two Reference Materials and Bulk Normalization as $100 \%(\mathrm{wt})$.......................................60

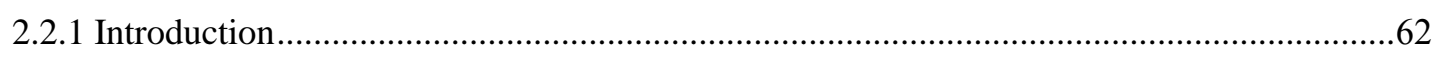

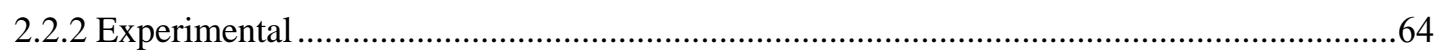

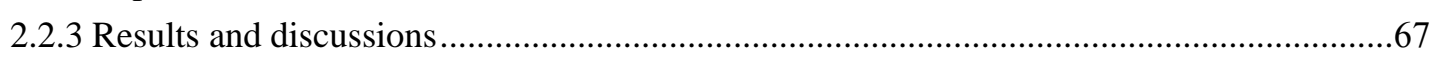

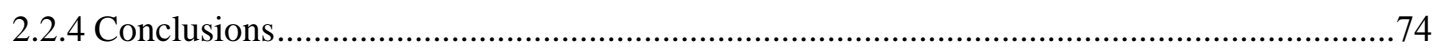

Chapter 3 Further improvement the capability of LA-ICP-MS: From sample preparation techniques to plasma enhancement

Chapter 3.1 Comparison of Ultrafine Powder Pellet and Flux-free Fusion Glass for Bulk Analysis of

Granitic Rock Samples by Laser Ablation-Inductively Coupled Plasma-Mass Spectrometry ..............81

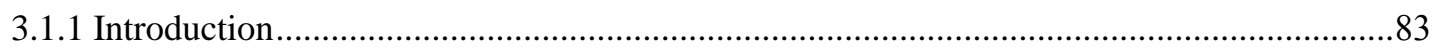

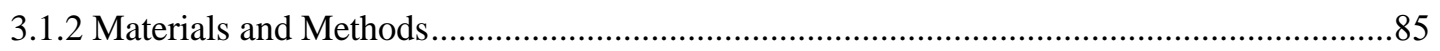

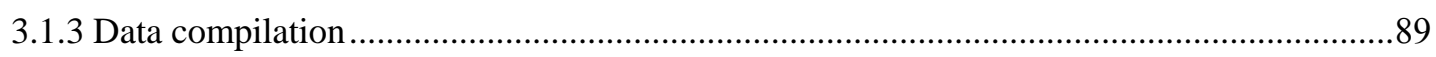

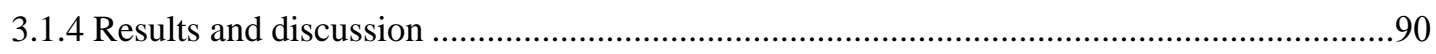

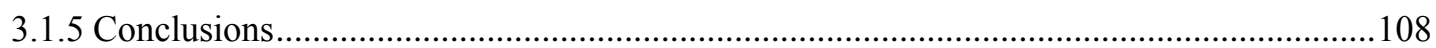

Chapter 3.2 Signal enhancement in LA-ICP-MS analysis by guard electrode and the addition of nitrogen and hydrogen into carrier gas: A perspective from experiment ............................................ 117

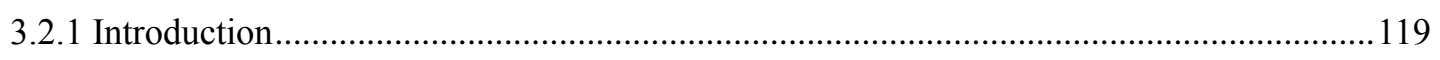

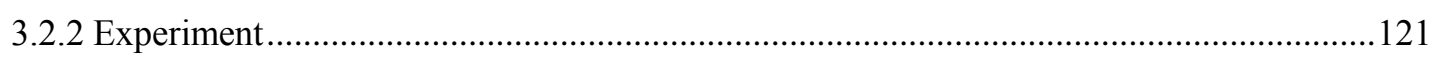

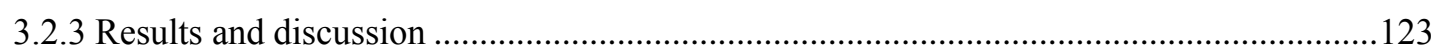

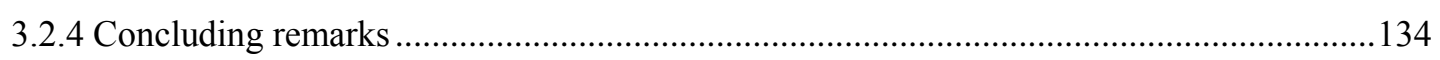

Chapter 4 Pushing the LA-ICP-MS analytical capability to sub- $\mu \mathrm{m}$ scale spatial resolution: Elemental fractionation studies and ablation behavior investigations .............................................143

Chapter 4.1 Elemental Fractionation Studies of $193 \mathrm{~nm}$ ArF Excimer Laser Ablation System at High

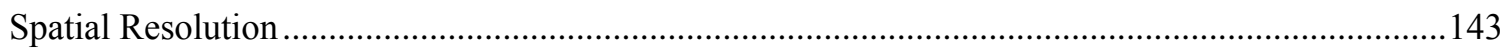

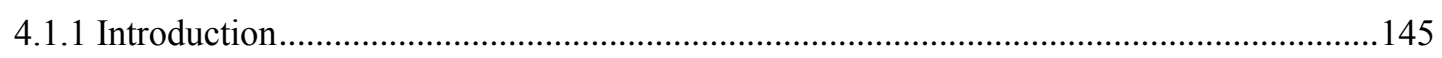

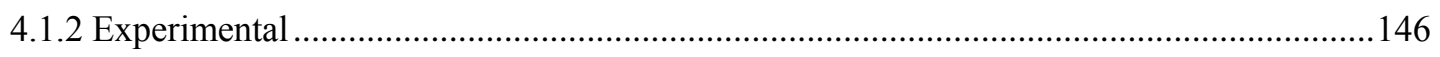

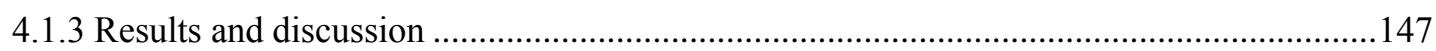

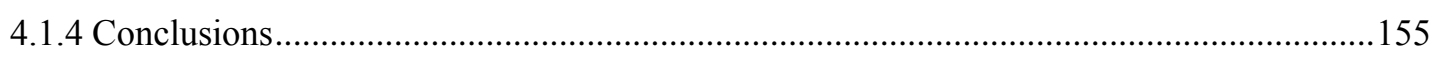


Chapter 4.2 Ablation Behaviors of 193nm ArF Excimer Laser for Selected Sample Substrates 162

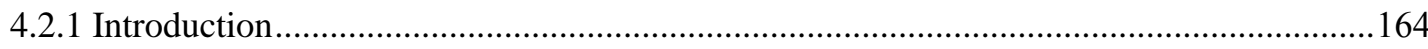

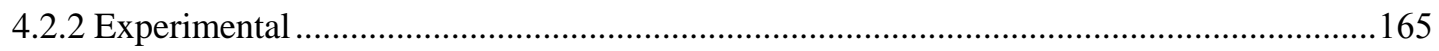

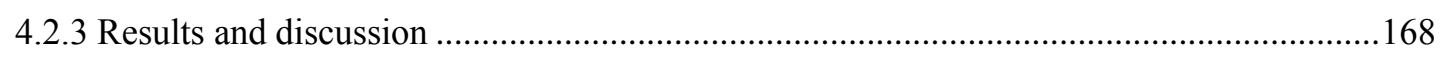

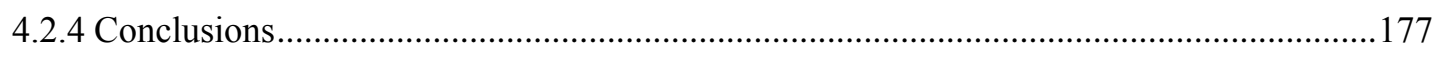

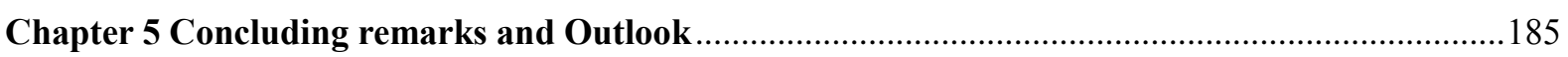

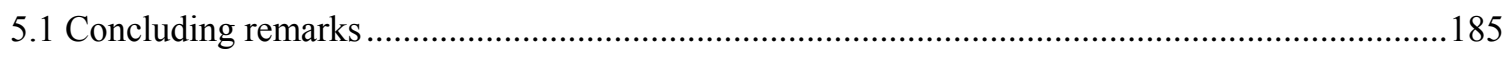

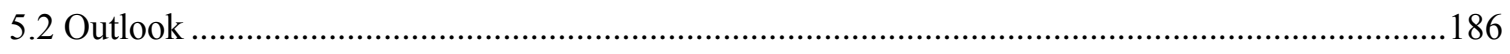

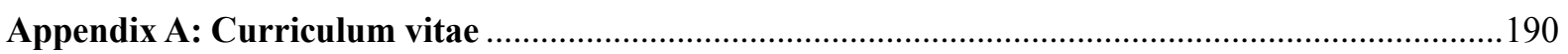

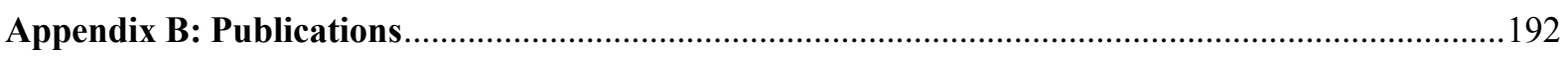

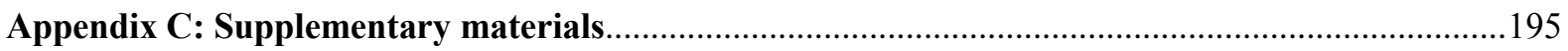




\section{Chapter 1 Introduction}

\subsection{A brief introduction of LA-ICP-MS}

Laser ablation-inductively coupled plasma-mass spectrometer (LA-ICP-MS) is a powerful micro-analytical technology by combining a laser ablation system (in situ solid sampling) to the inductively coupled plasma-mass spectrometer (ICP-MS) (signal detection). LA-ICP-MS begins with a laser beam focused on the sample surface to generate fine particles - a process known as "Laser Ablation". The ablated particles are then transported to the secondary excitation source (ICP) for atomization and ionization. The charged ions in the plasma torch are subsequently introduced to a mass spectrometer detector for both elemental and isotopic analysis.

Gray (1985) firstly demonstrated that a laser ablation system could be coupled to the inductively coupled plasma-mass spectrometer (ICP-MS) for in situ elemental analysis of solid samples. After 30 years of development, LA-ICP-MS has proven to be an extremely valuable analytical tool in Earth science. Its capability of multi-element analysis, ultra-high sensitivity, speed, and modern cost have led it to widespread applications including glass, mineral, inclusion micro-analysis, geochronology (e.g. U-Pb system), isotopic compositions (e.g. Li, Sr, Nd, Hf, Pb) and 2D/3D elemental mapping. Nowadays LA-ICP-MS is considered as an essential microanalysis technique in the geoanalytical laboratory.

\subsection{Scope and structure of the thesis}

Even though the great success has been achieved in diverse fields; there are still some aspects that need further investigations for the improvement of LA-ICP-MS capability. Among them, most critical are: Laser ablation process, Elemental fractionation, Sample preparation technique (for bulk analysis), Data reduction protocol, Strengthening plasma, Development of reference material, etc. 
There are some of the above-listed topics involved in this thesis corresponding to three individual chapters. These include:

(1) Data reduction protocol based on bulk normalization as $100 \%$ (wt), which is implemented within Iolite software package;

(2) Further improvement of LA-ICP-MS capability from sample preparation technique to strengthening plasma;

(3) Pushing the LA-ICP-MS analytical capability to sub-micron spatial resolution.

The thesis starts with an introductory chapter for a briefly description of the slope and structure of the thesis, then comes three scientific chapters that covering the Ph.D. projects. Each scientific chapter comprises of two publications or manuscripts. Thus a total of six publications or manuscripts are included in this thesis. Conclusion remarks and Outlook as an additional chapter (at the end) are to summary the major contributions of this thesis as well as outlook the nearby future of LA-IC-MS.

\subsection{Individual manuscript and Author contributions}

Chapter 2 regards to the development of data reduction protocols for elemental analysis, which is based on bulk normalization as $100 \%(w \mathrm{t})$ and implemented within Iolite software package. The quality of LA-ICP-MS data depends not only on the instrumentation but also the protocols used for data processing. This chapter includes two manuscripts as follows,

\section{Chapter 2.1}

Shitou Wu and Klaus Simon. (2017)

LA-ICP-MS transient signal quantification of NIST, MPI-DING, USGS, and CGSG glass reference materials by Ratioing, Standardization, and Normalization (RSN) (submitted to "Geostandards and Geoanalytical Research").

A quantitative reduction strategy consisting of Ratioing, Standardization, and Normalization (RSN) was proposed to process the LA-ICP-MS transient signal of NIST, MPI-DING, USGS 
and CGSG glass reference materials. The RSN strategy allows the quantitative reduction without knowing the concentration of internal standard prior to LA-ICP-MS analysis.

In this manuscript, I carried out the experiments and wrote the manuscript. Klaus Simon made the algorithms and compiled the code script for accomplishing the proposed protocol. He also improved the manuscript.

\section{Chapter 2.2}

Shitou Wu, Yaping Wang, and Chunxue Xu. (2017)

Laser ablation-inductively coupled plasma-mass spectrometer: A quantification strategy based on two reference materials and bulk normalization as 100\% (wt). Chinese Journal of Analytical Chemistry. 45(7), 965-972.

A quantification strategy based on two reference materials (NIST610 and StHs6/80-G) and bulk normalization as $100 \%(\mathrm{wt})$ were proposed to reduce LA-ICP-MS transient signals, which eliminates the deficiencies encountered with the quantification strategy using single reference material, such as the extremely low content or large uncertainty of some elements.

In this manuscript, I proposed the quantification strategy and conducted the experiment and wrote the manuscript. The other two authors edited and improved the manuscript.

Chapter 3 refers to the bulk analysis of granite by LA-ICP-MS and strengthening plasma with the addition of nitrogen and hydrogen into plasma in combination with guard electrode. These two projects lead to the further improvement of LA-ICP-MS capability.

\section{Chapter 3.1}

Shitou Wu, Volker Karius, Burkhard C Schmidt, Klaus Simon and Gerhard Wörner. (2017)

Comparison of ultrafine powder pellet and flux-free fusion glass for bulk analysis of granitic rock samples by Laser Ablation-Inductively Coupled Plasma-Mass Spectrometry. (submitted to “Geostandards and Geoanalytical Research”).

Two approaches are developed that include an optimized wet-milling protocol for granites 
and a flux-free fusion protocol for producing homogeneous granitic glasses. Comparison of ultrafine powder pellet and flux-free fusion glass for LA-ICP-MS bulk analysis of granitic rock samples was carried out.

In this manuscript, I was involved in developing this idea and wrote the manuscript. Volker Karius supervised the wet-milling experiment and edited the manuscript. Burkhard C. Schmidt managed the flux-free fusion experiment and modified the manuscript. Klaus Simon helped with the interpretation of the data. Gerhard Wörner and Volker Karius initially suggested the idea and improved the manuscript.

\section{Chapter 3.2}

Shitou Wu and Klaus Simon. (2017)

Signal enhancement in LA-ICP-MS analysis by guard electrode and the addition of nitrogen and hydrogen into carrier gas: A perspective from experiment (in preparation for "Journal of Analytical Atomic Spectrometry").

The signal enhancement in LA-ICP-MS by using guard electrode and the addition of small amount nitrogen/hydrogen into carrier gas was investigated. The plasma strength was evaluated under different experimental conditions.

In this manuscript, I designed and carried out the experiments and wrote the manuscript. Klaus Simon helped with the interpretation of the data and edited the manuscript.

Chapter 4 involves the pushing the LA-ICP-MS analytical capability to sub-micro spatial resolution. Elemental fractionation and laser ablation rate are the two bottlenecks that hamper this application. This chapter covers two manuscripts, which are focusing on elemental fractionation at high spatial resolution and laser ablation rate, respectively.

\section{Chapter 4.1}

Shitou Wu, Yaping Wang, Chunxue Xu and Jihai Yuan. (2016)

Elemental fractionation studies of $193 \mathrm{~nm}$ ArF excimer laser ablation system at high spatial resolution. Chinese Journal of Analytical Chemistry. 44(7), 1035-1041. 
Limits of detection (LODs), mass load effect, downhole fractionation and matrix effect of $193 \mathrm{~nm}$ ArF excimer laser ablation system at high spatial resolution were systematically investigated. Trace elements in GSD-1G, StHs6/80-G, and NIST612 were analyzed at $10 \mu \mathrm{m}$ spot size.

For this study, I proposed the idea, conducted the experiments, and wrote the manuscript. Other co-authors helped with the interpretation of the data and improved the manuscript.

\section{Chapter 4.2}

Shitou Wu, Chunxue Xu, Klaus Simon, Yilin Xiao and Yaping Wang. (2017)

Ablation behaviors of 193nm ArF excimer laser system for the selected substrates. Rock and Mineral Analysis. Accepted. (in Chinese with English abstract).

Ablation behaviors of 193 ArF excimer laser for silicate glasses, common minerals, and powder pellets were investigated. Ablation rates influenced by laser parameters (including spot size, energy density, and laser frequency) were evaluated. Data for ablation rates of 43 different substrates are presented.

In this manuscript, I designed the experiment and wrote the manuscript. Klaus Simon helped to collect the topographic images of laser craters. Yilin Xiao provided samples. Other co-authors helped with the interpretation of the data and improved the manuscript.

"Nothing can be obtained in geochemistry without careful analytical work."

by C.J.Allegre 


\section{Chapter 2 Quantification of LA-ICP-MS transient signals based on Iolite software package}

Chapter 2.1 LA-ICP-MS Transient Signal Quantification of NIST, MPI-DING, USGS and CGSG Glass Reference Materials by Ratioing, Standardization, and Normalization (RSN)

Shitou Wu*, Klaus Simon

Geowissenschaftliches Zentrum, Göttingen Universität, Göttingen 37077, Germany

Corresponding author E-mail address: wushitou111@hotmail.com

Fax: +49 $55139-23982$ 
Abstract: A quantitative reduction strategy consisting of Ratioing, Standardization, and Normalization (RSN) was proposed to process the LA-ICP-MS transient signal of NIST, MPI-DING, USGS and CGSG glass reference materials. The RSN strategy allows the quantitative reduction without knowing the concentration of internal standard prior to LA-ICP-MS analysis. The down-hole fractionations of the investigated glasses in initial 5-35s ablation period were insignificant and independent of the chosen internal standards under the given laser conditions. The analytical accuracy obtained from internal standardization was affected by the given value of the internal standard. Contrarily, accuracy obtained from RSN strategy was independent of the chosen internal standard. Matrix effects between NIST610 and geological glasses were negligible. Imprecise certified values of several elements were identified. The prefer values reported in this study are the better-estimated values for these elements. Laser spot size could be down to $15 \mu \mathrm{m}$ where the applicability of RSN strategy was still acceptable. The short- and long-term precision (359 repetition analysis over three years) illustrated that either instrument conditions or the capability of RSN strategy were approvingly acceptable. The simplicity and applicability of RNS strategy in comparison with internal standardization strategy make it suitable for quantitative reduction for silicate glasses.

Keywords: Laser ablation inductively coupled plasma mass spectrometry; quantitative reduction strategy; downhole fractionation; certified value; silicate glass 


\subsubsection{Introduction}

Laser ablation inductively coupled plasma mass spectrometry (LA-ICP-MS), as a spatially resolved technique, has been widely used for chemical analysis in geochemistry (Liu et al., 2013), such as in-situ mineral analysis (Cruz-Uribe et al., 2016; Stead et al., 2017), elemental 2D imaging (Raimondo et al., 2017; Ubide et al., 2015) and rock bulk analysis (Garbe-Schönberg and Müller, 2014; He et al., 2016) etc. In the past decades, the physical and chemical mechanisms associated with ablation procedure (Russo et al., 2013; Russo et al., 2004), aerosol transportation (Koch et al., 2008; Kovacs and Günther, 2008) and ICP ionization process (Fietzke and Frische, 2016; Wang et al., 2006) have been studied thoroughly and significant improvement has been achieved. Quantitative reduction strategy plays a crucial role in the transmission of transient signals to element concentration, and it is an essential issue for the data quality assurance since the emergence of LA-ICP-MS (Jackson, 2008). The most popular reduction strategy is the internal standardization strategy described by Longerich et al.(1996). In general, the quantification strategy consists of three main aspects that need to be corrected or calibrated: elemental fractionation, ablation mass yields, and ICP mass response.

Elemental fractionation has been considered as a serious limitation for LA-ICP-MS since early 1980s (Gray, 1985), which is normally used to summarize all the non-stoichiometric effects occurring during ablation procedure (Mank and Mason, 1999), aerosol transportation (Koch et al., 2002) and ICP ionization process (Guillong and Günther, 2002; Kroslakova and Günther, 2007). As the introduction of $193 \mathrm{~nm}$ ArF laser, the particle size induced fractionation existing in aerosol transportation and ICP process has been significantly reduced (Guillong et al., 2003), however, the thermal induced fractionation during the ablation process (the so-called "downhole fractionation") still remains. Several approaches have been suggested to diminish the downhole fractionation, such as mathematical model correction (Horn et al., 2000; Chad Paton et al., 2010), multiple internal standardization (Jackson, 2008), shallower ablation crater (J. G. Mank and R. D. Mason, 1999), raster mode 
ablation (Jochum et al., 2014) and using femtosecond laser (Horn and von Blanckenburg, 2007). Although Günther and Heinrich (1999) demonstrated that down-hole fractionations induced by the $193 \mathrm{~nm}$ laser were insignificant, particularly in the first 40s ablation period, a systematical investigation of downhole fractionation needs to be accomplished prior to any quantitative strategy applied in practical calibration.

The change of ablation mass due to the matrix-dependent laser energy absorption could be corrected either via an internal standard or bulk normalization as $100 \% \mathrm{~m} / \mathrm{m}$. Longerich et al.(1996) reported the internal standardization strategy where a "natural occurring internal standard" is used to correct the ablation mass for each analysis. Halicz and Günther (2004) accomplished silicate glass quantification by assuming the sum of oxide as $100 \% \mathrm{~m} / \mathrm{m}$ to correct the ablation mass and using the simultaneous liquid calibration strategy. This approach combined with a solid external reference material (NIST610 or BCR-2G) was successfully used for analysis of GSA-1G, GSC-1G, GSD-1G, and GSE-1G by Guillong et al. (2005). The approach of bulk normalization as $100 \% \mathrm{~m} / \mathrm{m}$ is practical compared to internal standardization due to its simplicity without requiring the internal standard content prior to LA-ICP-MS analysis, however there are still some uncertainties that need to be understood well, including the requirement of state of multivalent elements, the assumption of missed components, and the accuracy of single point calibration et al. Some of these have been discussed in the previous works by Gagnon et al. (2008) and Liu et al. (2008).

ICP mass response could be obtained by analysis of an external certified reference material, which has been already successfully used for the trace element and isotopic ratio measurement in geological samples (Stoll et al., 2008; Zhang et al., 2014). Usually two questions come out when the external certified reference material is applied, namely matrix effect and the quality of reference material (Luo et al., 2007) (including homogeneity, element concentration and uncertainty of certified values et al.). NIST series glass has been widely accepted as external reference material due to the high abundance of trace elements, 
however some literature (Czas et al., 2012; Liu et al.,, 2008; Sylvester, 2008) reported the existing matrix effect relative to geological samples and the potential heterogeneity distribution of several elements (Pearce et al., 1997). For these reasons, the MPI-DING, USGS, and CGSG geological glasses were produced. Although those reference materials are matrix matched well with geological samples, the nature of some low concentration elements makes them mostly work as the secondary calibration materials.

In this paper, a reduction strategy that consists of Ratioing, Standardization, and Normalization (RSN) was proposed to accomplish LA-ICP-MS quantification of NIST, MPI-DING, USGS, and CGSG glass reference materials. The RSN strategy allows the correction/calibration of downhole fractionation, ablation mass yields, and ICP mass response. Although the RSN strategy uses an internal standard, it could achieve the quantitative reduction without knowing the concentration of internal standard prior to LA-ICP-MS analysis. The RSN strategy was performed in Iolite 3.4 environment with an in-house compiled data reduction scheme (DRS). We assessed the downhole fractionation (induced by the 193nm ArF laser) in glass reference materials. The evaluation and comparison of internal standardization strategy and RSN strategy were carried out by quantification of ML3B-G and KL2-G based on six internal standards (including ${ }^{27} \mathrm{Al},{ }^{29} \mathrm{Si},{ }^{43} \mathrm{Ca},{ }^{65} \mathrm{Cu},{ }^{88} \mathrm{Sr}$, and ${ }^{140} \mathrm{Ce}$ ). Three external reference materials that include NIST610, GSD-1G and StHs6/80-G were used as for standardizing other glass reference materials. The influence of spatial resolution (laser spot size) to the RSN strategy was also investigated. Meanwhile, the short- and long-term analytical precision (data reduced by RSN strategy) was discussed. The applicability of RSN strategy was assessed by a series of currently available glass reference materials (NIST, MPI-DING, USGS, and CGSG). The certified values of investigated glass reference materials were evaluated and the preferred values were reported. 


\subsubsection{Experimental}

\subsubsection{Instrumentation}

RESOlution M-50 ablation system (ASI, Australia) combined with an Element 2 sector field ICP-MS (ThermoScientific, USA) were used in this study. A 'squid' smooth device was used to improve the signal precision (Müller et al., 2009). Helium was employed as ablation environment gas to enhance the sensitivity (Eggins et al., 1998; Günther and Heinrich, 1999). The instrument conditions were optimized by continuous ablating NIST 612 in raster mode to achieve the highest ${ }^{139} \mathrm{La}$ intensity while keeping the U/Th around 1 , oxide $\left(\mathrm{ThO}^{+} / \mathrm{Th}^{+}\right)$and secondary ion production $\left(\mathrm{Ca}^{2+} / \mathrm{Ca}^{+}\right)$lower than $0.5 \%$. The detailed instrument conditions and measurement parameters of LA-ICP-MS are summarized in Table 1.

Table1 Operation conditions of LA-ICP-MS system

\begin{tabular}{ll}
\hline Laser ablation system & \\
\hline Laser type & ArF Excimer \\
Wavelength & $193 \mathrm{~nm}$ \\
Pulse time & $20 \mathrm{~ns}$ \\
Energy density & around $5.0 \mathrm{~J} \mathrm{~cm}^{-2}$ \\
Repetition rate & $5 \mathrm{~Hz}$ \\
Ablation cell & Laurin Technic S-155 \\
Spot size & $10-130 \mu \mathrm{m}$ \\
Ablation gas flow $(\mathrm{He})$ & $0.65 \mathrm{~L} / \mathrm{min}$ \\
Ablation time & $35,120 \mathrm{~s}$ \\
\hline ICP-MS & \\
\hline ICP-MS & Element 2 \\
RF Power & $1400 \mathrm{~W}$ \\
Guard electrode & Floated (Pt) \\
Sample cone & Ni $(54605)$ \\
Skimmer cone & Ni $(543540.8 \mathrm{H})$ \\
Coolant gas flow (Ar) & $15.00 \mathrm{~L} / \mathrm{min}$ \\
Auxiliary gas flow (Ar) & $1.00 \mathrm{~L} / \mathrm{min}$ \\
Carrier gas flow (Ar) & $0.95 \mathrm{~L} / \mathrm{min}$ \\
Scan mode & E-scan \\
Segment duration & $10 \mathrm{~ms}$ \\
Detector & Dual (counting and analog) \\
Resolution (M/ $\mathrm{MM})$ & Low (around 300) \\
\hline
\end{tabular}




\subsubsection{Samples and Data Acquisition}

A series of glass reference materials that include NIST, MPI-DING, USGS, and CGSG were investigated in this study (Table 2). These glasses are mostly acceptable as LA-ICP-MS calibration materials and well characterized in previous studies. Reference values of USGS glass were cited from GeoReM database (http://georem.mpch-mainz.gwdg.de/). Reference values of CGSG-1 and CGSG-5 glass were obtained from Prof. Dr. Zhan Xiuchun (National Research Center for Geoanalysis, China). All others were cited from Jochum's literature (Jochum and Enzweiler, 2014; Jochum et al., 2006; Jochum et al., 2011), except $\mathrm{Nb}$ and Ta in NIST610, which were collected from Hu et al. (2008) (see Table 2). The glasses were firstly embedded in epoxy and then polished to a flat surface. Ultrasonic cleaning in water medium was performed before LA-ICP-MS analysis. Analysis sequence started with a calibration group that includes NIST610, StHs6/80-G, and GSD-1G, followed by three, five or ten repetitions of one glass reference material (treated as unknown), and then again calibration group. The analysis sequence consisting of a number of spot analyses was run in the automatic mode. A total of 15 sequences were run over three years (Appendix S1).

The aerosol from ablation may produce deposits across the sample surface so that a pre-ablation procedure (two laser pulses) was performed to avoid the potential influence caused by aerosol deposits and any other form of surface contamination. Element 2 produces a flat-top peak at low resolution with the flatness comprising about $20 \%$ of the entire peak. The central $5 \%$ of the peak (one point) were sampled to achieve short sweep time. Element 2 was adjusted to the fast speed mode. The sweep time from the lowest (7) to highest (238) mass was optimized by carefully adjusting magnetite setting time without deteriorating the counting efficiency. Each individual analysis incorporated a background acquisition of $20 \mathrm{~s}$ (gas blank) followed by a $35 \mathrm{~s}$ ablation data acquisition, which consists of a total 55 sweeps. The ICP-MS method is shown in Appendix S2. The detailed information of scanned isotopes, oxide and oxide coefficient are shown in Appendix S3. 
Chapter 2.1 LA-ICP-MS Transient Signal Quantification of NIST, MPI-DING, USGS and CGSG Glass Reference Materials by Ratioing, Standardization, and Normalization (RSN)

Table 2 Detailed information of the investigated glass reference materials in this study

\begin{tabular}{|c|c|c|c|c|}
\hline Sample & Supplier & Category & Matrix & Source of reference values \\
\hline NIST610 & NIST & CRM & Synthetic silicate & Jochum et al. 2011 and $\mathrm{Hu}$ et al.2008a \\
\hline NIST612 & NIST & CRM & Synthetic silicate & Jochum et al. 2011 \\
\hline NIST614 & NIST & CRM & Synthetic silicate & Jochum et al. 2011 \\
\hline StHs6/80-G & MPI-Chemie & CRM & Natural andesite & Jochum et al. 2006 \\
\hline ATHO-G & MPI-Chemie & CRM & Natural rhyolite & Jochum et al. 2006 \\
\hline T1-G & MPI-Chemie & CRM & Natural quartz-diorite & Jochum et al. 2006 \\
\hline ML3B-G & MPI-Chemie & CRM & Natural basalt & Jochum et al. 2006 \\
\hline KL2-G & MPI-Chemie & CRM & Natural basalt & Jochum et al. 2006 \\
\hline GOR128-G & MPI-Chemie & CRM & Natural komatiite & Jochum et al. 2006 \\
\hline GOR132-G & MPI-Chemie & CRM & Natural komatiite & Jochum et al. 2006 \\
\hline BCR-2G & USGS & $\mathrm{RM}$ & Natural basalt & GeoReM database \\
\hline BHVO-2G & USGS & $\mathrm{RM}$ & Natural basalt & GeoReM database \\
\hline BIR-1G & USGS & $\mathrm{RM}$ & Natural basalt & GeoReM database \\
\hline GSD-1G & USGS & $\mathrm{RM}$ & Synthetic basalt & GeoReM database \\
\hline CGSG-1 & NRCG & CRM & Natural basalt & X.Zhan per.comm \\
\hline CGSG-2 & NRCG & CRM & Natural nepheline syenite & Jochum and Enzweiler 2014 \\
\hline CGSG-4 & NRCG & CRM & Natrural soil & Jochum and Enzweiler 2014 \\
\hline CGSG-5 & NRCG & CRM & Natural andesite & X.Zhan per.comm \\
\hline
\end{tabular}

NIST: National Institute of Standards and Technology, USA; MPI-Chemie: Max Plank Institute for Chemistry, Germany; USGS: United States Geological Survey, USGS; NRCG: National Research Center for Geoanalysis, China; CRM: Certified Reference Material; RM: Reference Materia

\subsubsection{RSN Strategy and Iolite software}

The quantification strategy consists of three parts, including Ratioing, Standardization, and Normalization (RSN).

\section{Ratioing}

Elemental intensities collected in each sweep were firstly normalized to the internal standard.

The intensity ratios were treated as the basic unit for the quantification algorithms. The intensity ratios, instead of absolute intensity, could improve the analytical precision since mass spectrometry measure precisely for the relative value rather than absolute one.

$$
\frac{i_{e l}}{i_{i s}}, \frac{c_{e l}}{c_{i s}}
$$




\section{Standardization}

The standardization algorithm is used for the calculation of the elemental concentration ratios, which is based on a certified reference material as a calibration standard. The standardization algorithm is shown in Eq.1 (modified from Longerich et al. (1996)).

$$
\left.\frac{c_{e l}}{c_{i s}}\right|_{S A M}=\left.\left.\left.\frac{i_{e l}}{i_{i s}}\right|_{S A M} * \frac{i_{i s}}{i_{e l}}\right|_{R M} * \frac{c_{e l}}{c_{i s}}\right|_{R M}
$$

\section{Normalization}

Based on the assumption of bulk oxide as $100 \% \mathrm{~m} / \mathrm{m}$ (as shown in Eq.2), we could correct the change of ablation mass for each analysis and give the constraint to calculate the absolute concentration.

$$
\left.\sum_{n=1}^{N} \quad \frac{c_{e l}^{n}}{c_{i s}}\right|_{S A M} * f_{e l}=\frac{100 \% m / m-\epsilon}{c_{i s}}
$$

Combining the Eq.1 and Eq.2 obtains Eq.3, as shown below.

$$
c_{e l}^{c o r}{ }_{\mid S A M}=\left.\left.\left.\frac{i_{e l}}{i_{i s}}\right|_{S A M} * \frac{i_{i s}}{i_{e l}}\right|_{R M} * \frac{c_{e l}}{c_{i s}}\right|_{R M} * \frac{100 \% m / m-\epsilon}{\left.\left.\left.\sum_{n=1}^{N} \frac{i_{e l}^{n}}{i_{i s}}\right|_{S A M} * \frac{i_{i s}^{n}}{i_{e l}}\right|_{R M} * \frac{c_{e l}^{n}}{c_{i s}}\right|_{R M} * f_{e l}}
$$

where $i$ and $c$ represent the intensity and concentrations of analyzed elements; $e l$ and $i s$ represent the targeted element and internal standard; $S A M$ and $R M$ represent target samples and reference material; $f$ represents the corresponding oxide coefficient; $\epsilon$ is the missed components.

Iolite provides a powerful framework for transient data processing and interpretation, especially for LA-ICP-MS (Paton et al., 2011). The RSN strategy was accomplished in Iolite3.4 software and a data reduction scheme (modified from "Trace element", see Appendix S2) was compiled to realize the quantification algorithms. The initial five and last 
three sweeps were excluded from the data integration to avoid the influence of unstable intensity. In practical, the reduction procedure is shown as follows.

The intensity was firstly subtracted from the gas blank and then normalized to the internal standard to make intensity ratio. The instrumental drift was corrected by using a linear interpolation based on the variations of NIST 610 intensity ratios. An arbitrary value (such as $40 \% \mathrm{~m} / \mathrm{m}$ ) was given to the internal standard. The internal standardization (calibrated with an external reference material) was carried out to produce the raw element concentration data. These raw data were converted to oxide concentration by multiplying oxide coefficient, and then the sum of oxide was scaled to $100 \% \mathrm{~m} / \mathrm{m}$ to calculate the internal standard concentration. The factor (ratio of an arbitrary value and the calculated concentration of internal standard) was used to correct the raw data to final data.

The crucial uncertainties in RSN strategy are related to the normalization part (bulk normalization as $100 \% \mathrm{~m} / \mathrm{m}$ ), which include (1) the state of multivalent elements, (2) the missed components, and (3) the accuracy of single point calibration. The first two have been discussed in previous works by Guillong et al. (2005), Gagnon et al., (2008), and Liu et al., (2008). Generally, an assumption of a multivalent state of high abundance element may lead an uncertainty for the bulk normalization as $100 \% \mathrm{~m} / \mathrm{m}$. Iron is the only multivalent element as a major component occurring in silicate rocks. Here, four assumptions of iron valence were evaluated (Fig.1). As shown in Fig.1, the maximum uncertainty was only $1 \%$ with an assumption of $\mathrm{Fe}^{2+} / \mathrm{Fe} \_\mathrm{t}$ as 0.5 for $\mathrm{FeO} \_t$ (total $\mathrm{Fe}$ content expressed as $\mathrm{FeO}$ ) up to $15 \% \mathrm{~m} / \mathrm{m}$. This $1 \%$ uncertainty is ignorable considering around $10 \%$ analytical uncertainty for trace element analysis with LA-ICP-MS technique. 


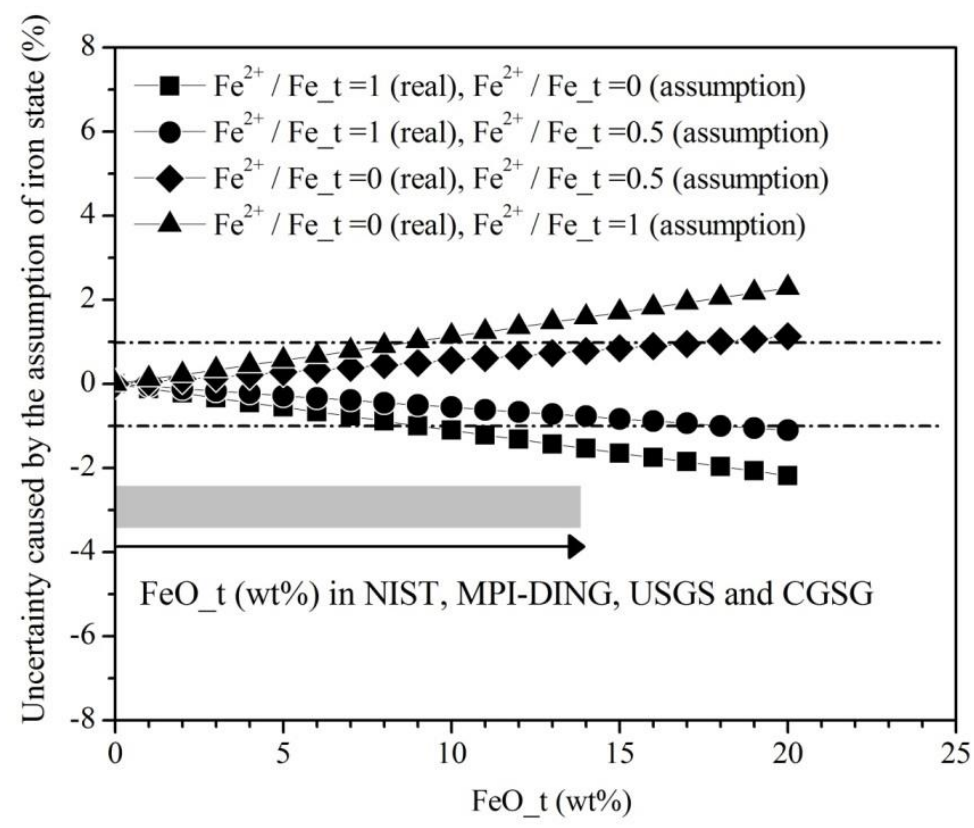

Fig. 1 Uncertainties caused by the assumption of iron valence.

The missed components that include $\mathrm{H}, \mathrm{C}, \mathrm{N}, \mathrm{F}, \mathrm{Cl}, \mathrm{Br}$ and I (those parts could not be measured or accurately measured by LA-ICP-MS) will introduce uncertainty for the bulk normalization as $100 \% \mathrm{~m} / \mathrm{m}$ (Liu et al., 2008). The percentage of the missed components could lead to the same degree uncertainty (Fig.1 in Appendix S4). A proper assumption of the missed components is a prerequisite for the accurate quantification of hydrous or high halogen minerals (the missed components could be up to $10 \% \mathrm{~m} / \mathrm{m}$ ). Here we calculated the sum of oxide $\left(\mathrm{Fe}^{2+} / \mathrm{Fe}_{-} \mathrm{t}\right.$ as 0.5$)$ for the investigated glass reference materials (Table 1 in Appendix S4). The missed components in the investigated glass samples are generally lower than $1 \%$ (except CGSG-5), which demonstrates that the assumption of oxide as $100 \% \mathrm{~m} / \mathrm{m}$ for the glass reference materials is appropriate.

In most cases, a single external reference material was used for LA-ICP-MS calibration, so that any uncertainty in single point calibration will propagate to the bulk normalization part, especially for the major elements. Fig. 2 shows the calibration lines of the selected elements (covering from major to trace elements). The usage of NIST glasses to calibrate geological glass may result in imprecise values for some major elements ( $\mathrm{Mg}, \mathrm{Fe}, \mathrm{Ti}, \mathrm{Mn}$ and $\mathrm{K})$ due to 
their low concentration (nearby the limit of detection), and thus would cause the uncertainty for bulk normalization as $100 \% \mathrm{~m} / \mathrm{m}$. Meanwhile, reference materials with elements concentration lower than the detection limit (like Cs in BIR-1G, Th in GOR130-G and GOR128-G) are not suitable as the external calibration materials, especially for those elements calibration.
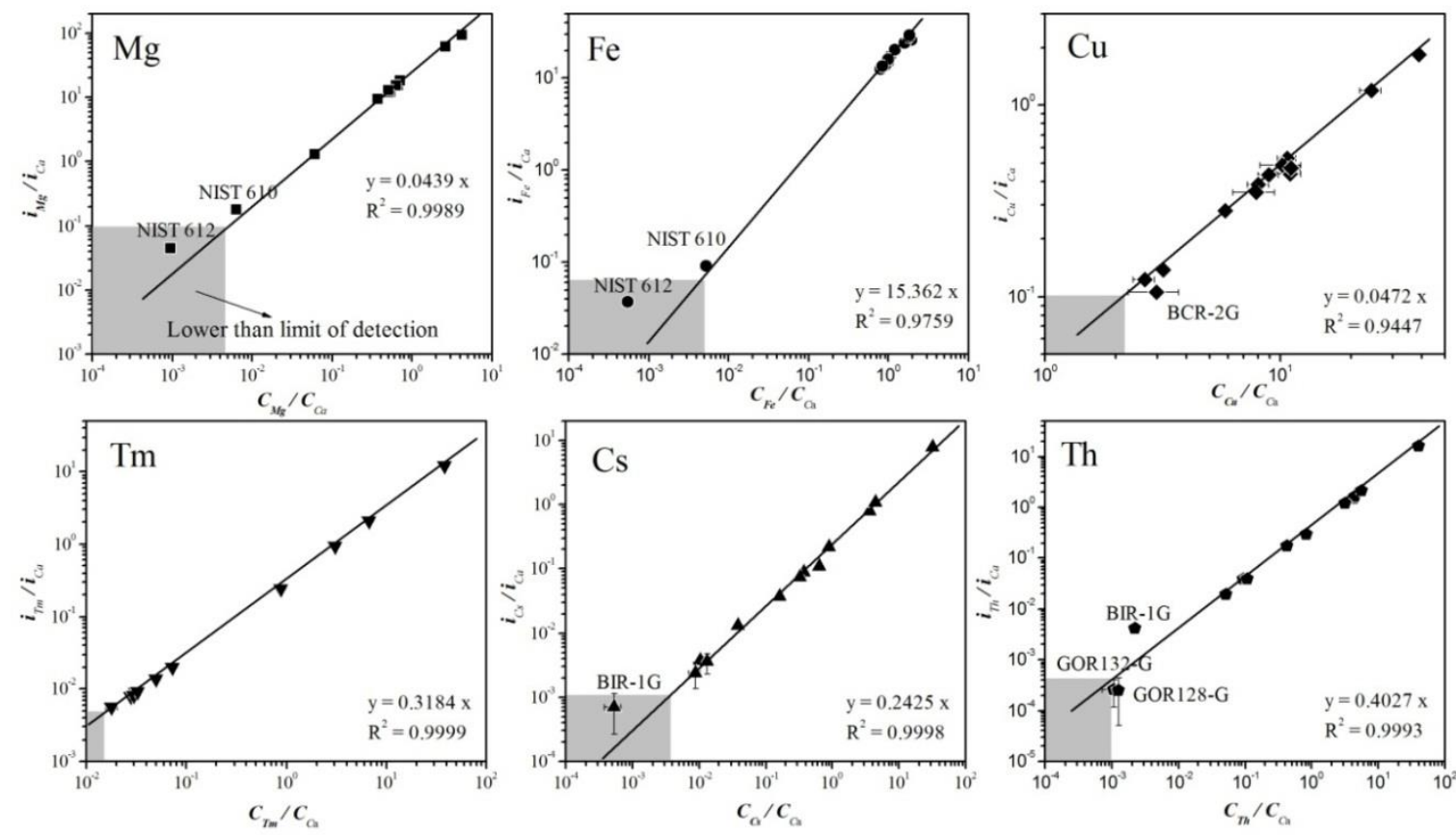

Fig. 2 Calibration line of selected elements including $\mathrm{Mg}, \mathrm{Fe}, \mathrm{Cu}, \mathrm{Tm}, \mathrm{Cs}$ and Th. Grey zone indicates the detection limits (calculated with three times of standard deviation of gas blank intensity). The intensity ratios used here are corrected from the effect of instrument drift.

\subsubsection{Data compilations}

A total of 15 analysis sequences were run over three years. The detailed information of analysis sequence was shown in Appendix S1. Each glass was measured by a number of repetitions. The data were calculated with NIST610, StHs6/80-G, and GSD-1G as external reference materials (Table 3). The prefer values were compiled all the data standardized with different external reference materials, except some elements occurring with extreme low contents (Ti, Fe, Mn, Mg, K, and P in NIST610, Be, Mo and Sb in StHs6/80-G) or under/overestimated certified values (Cr, Ni in StHs6/80-G and Sn, Sb in GSD-1G).

“*” means the data are given as information value

“-” represent the data not given or the data lower than detection limit. 
Chapter 2.1 LA-ICP-MS Transient Signal Quantification of NIST, MPI-DING, USGS and CGSG Glass Reference Materials by Ratioing, Standardization, and Normalization (RSN)

Table $3 \mathrm{a}$

Element concentrations of NIST610 obtained from RSN strategy with NIST 610, StHs6/80-G and GSD-1G as external reference materials. Values are in units of $\mu \mathrm{g} / \mathrm{g}$ except for major elements which are specified in weight percent $(\% \mathrm{~m} / \mathrm{m})$. Standard deviation $(1 \mathrm{~s})$ stems from 146 measurements.

\begin{tabular}{|c|c|c|c|c|c|c|c|c|c|c|c|c|c|c|}
\hline \multirow[b]{2}{*}{ Elements } & \multicolumn{2}{|c|}{ Reference value } & \multicolumn{2}{|l|}{ NIST610 } & \multirow[b]{2}{*}{$\operatorname{RSD}(\%)$} & \multicolumn{3}{|c|}{ StHs6/80-G } & \multicolumn{3}{|l|}{ GSD-1G } & \multicolumn{3}{|c|}{ Prefer value } \\
\hline & Value & Uncertainty & Average & $1 \mathrm{~s}$ & & Average & $1 \mathrm{~s}$ & $\operatorname{RSD}(\%)$ & Average & $1 \mathrm{~s}$ & $\operatorname{RSD}(\%)$ & Average & $1 \mathrm{~s}$ & $\operatorname{RSD}(\%)$ \\
\hline $\mathrm{SiO} 2$ & 69.7 & 0.5 & 70.7 & 0.0675 & 1.38 & 70.5 & 0.506 & 1.21 & 70.2 & 0.629 & 0.73 & 70.5 & 0.487 & 1.14 \\
\hline $\mathrm{TiO} 2$ & 0.0754 & 0.0017 & 0.0764 & 0.0009 & 1.38 & 0.086 & 0.0023 & 14.06 & 0.0826 & 0.0021 & 9.54 & 0.0845 & 0.0028 & 12.12 \\
\hline $\mathrm{Al} 2 \mathrm{O} 3$ & 1.95 & 0.04 & 1.98 & 0.0044 & 1.38 & 2.1 & 0.0315 & 7.82 & 2.04 & 0.035 & 4.39 & 2.04 & 0.0598 & 4.54 \\
\hline $\mathrm{FeO}(\mathrm{t})$ & 0.0589 & 0.0012 & 0.0598 & 0.0012 & 1.42 & 0.0644 & 0.0024 & 9.33 & 0.0676 & 0.003 & 14.67 & 0.0658 & 0.0031 & 11.62 \\
\hline $\mathrm{MnO}$ & 0.0573 & 0.0017 & 0.0581 & 0.0002 & 1.38 & 0.0625 & 0.0015 & 9.02 & 0.0629 & 0.0013 & 9.71 & 0.0627 & 0.0014 & 9.32 \\
\hline $\mathrm{MgO}$ & 0.0716 & 0.0048 & 0.0726 & 0.0005 & 1.38 & 0.0872 & 0.0016 & 21.77 & 0.0865 & 0.0018 & 20.73 & 0.0869 & 0.0018 & 21.32 \\
\hline $\mathrm{CaO}$ & 11.4 & 0.2 & 11.6 & 0.0551 & 1.38 & 11.8 & 0.38 & 3.32 & 11.9 & 0.514 & 4.07 & 11.7 & 0.377 & 2.82 \\
\hline $\mathrm{Na} 2 \mathrm{O}$ & 13.4 & 0.3 & 13.6 & 0.0371 & 1.38 & 13.2 & 0.217 & -1.66 & 13.5 & 0.231 & 1.01 & 13.4 & 0.259 & 0.18 \\
\hline $\mathrm{K} 2 \mathrm{O}$ & 0.0559 & 0.0025 & 0.0567 & 0.0004 & 1.38 & 0.0504 & 0.0028 & -9.88 & 0.0497 & 0.0018 & -11.01 & 0.0501 & 0.0025 & -10.36 \\
\hline $\mathrm{P} 2 \mathrm{O} 5$ & 0.0946 & 0.0105 & 0.0961 & 0.0023 & 1.50 & 0.106 & 0.0069 & 11.74 & 0.0906 & 0.0067 & -4.22 & 0.0993 & 0.0101 & 4.88 \\
\hline $\mathrm{Be}$ & 476 & 31 & 483 & 8.31 & 1.37 & 1188 & 8820 & 149.49 & 538 & 31.6 & 12.98 & 506 & 34.9 & 6.36 \\
\hline B & 350 & 56 & 355 & 5.96 & 1.36 & 360 & 141 & 2.88 & 283 & 22.8 & -19.04 & 337 & 92.2 & -3.67 \\
\hline $\mathrm{Sc}$ & 455 & 10 & 461 & 1.71 & 1.38 & 517 & 31.3 & 13.66 & 510 & 16.1 & 12.02 & 495 & 32.8 & 8.75 \\
\hline $\mathrm{V}$ & 450 & 9 & 456 & 1.94 & 1.38 & 497 & 12.6 & 10.40 & 487 & 9.27 & 8.26 & 479 & 20.1 & 6.54 \\
\hline $\mathrm{Cr}$ & 408 & 10 & 414 & 3.83 & 1.44 & 610 & 39.4 & 49.58 & 438 & 20.6 & 7.41 & 424 & 18.4 & 4.01 \\
\hline Co & 410 & 10 & 416 & 1.57 & 1.38 & 425 & 13.4 & 3.63 & 432 & 10.1 & 5.42 & 424 & 11.7 & 3.30 \\
\hline $\mathrm{Ni}$ & 458.7 & 4 & 465 & 1.88 & 1.39 & 555 & 38.7 & 21.09 & 477 & 11.7 & 3.99 & 470 & 9.77 & 2.51 \\
\hline $\mathrm{Cu}$ & 441 & 15 & 447 & 2.14 & 1.38 & 473 & 16.1 & 7.20 & 456 & 15.7 & 3.29 & 459 & 16.9 & 4.02 \\
\hline $\mathrm{Zn}$ & 460 & 18 & 467 & 6.73 & 1.45 & 464 & 26.8 & 0.84 & 452 & 24.3 & -1.69 & 462 & 21.8 & 0.37 \\
\hline $\mathrm{Ga}$ & 433 & 13 & 439 & 1.84 & 1.39 & 458 & 25.1 & 5.69 & 435 & 13.2 & 0.54 & 445 & 19.3 & 2.72 \\
\hline $\mathrm{Rb}$ & 425.7 & 1 & 432 & 1.68 & 1.38 & 437 & 13.4 & 2.54 & 413 & 9.15 & -3.03 & 428 & 13.5 & 0.60 \\
\hline $\mathrm{Sr}$ & 515.5 & 1 & 523 & 2.22 & 1.39 & 539 & 14.1 & 4.53 & 556 & 13.1 & 7.88 & 538 & 17.2 & 4.31 \\
\hline $\mathrm{Y}$ & 462 & 11 & 468 & 1.58 & 1.38 & 493 & 15.5 & 6.63 & 522 & 18.7 & 12.89 & 492 & 25 & 6.44 \\
\hline Mo & 417 & 21 & 423 & 2.32 & 1.41 & 569 & 103 & 36.49 & 442 & 20.3 & 6.01 & 431 & 16.4 & 3.39 \\
\hline Sn & 430 & 29 & 436 & 2.46 & 1.40 & 448 & 63 & 4.15 & 362 & 12 & -15.83 & 442 & 45 & 2.78 \\
\hline $\mathrm{Sb}$ & 396 & 19 & 401 & 2.05 & 1.39 & 372 & 372 & -6.17 & 461 & 17.2 & 16.38 & 401 & 2.05 & 1.39 \\
\hline $\mathrm{Cs}$ & 366 & 9 & 371 & 1.91 & 1.38 & 406 & 17.1 & 10.92 & 375 & 8.91 & 2.40 & 385 & 19.7 & 5.12 \\
\hline $\mathrm{Ba}$ & 452 & 9 & 458 & 2.72 & 1.36 & 466 & 11 & 3.08 & 470 & 13 & 3.93 & 464 & 10.8 & 2.69 \\
\hline $\mathrm{La}$ & 440 & 10 & 446 & 2.51 & 1.38 & 462 & 13.4 & 5.07 & 481 & 13.7 & 9.33 & 462 & 17.6 & 4.89 \\
\hline $\mathrm{Ce}$ & 453 & 8 & 459 & 1.28 & 1.38 & 484 & 13.6 & 6.83 & 491 & 12.4 & 8.45 & 477 & 17.2 & 5.30 \\
\hline $\operatorname{Pr}$ & 448 & 7 & 454 & 2.83 & 1.37 & 482 & 13.4 & 7.48 & 484 & 12.1 & 8.07 & 472 & 17.3 & 5.42 \\
\hline $\mathrm{Nd}$ & 430 & 8 & 436 & 3.14 & 1.40 & 463 & 17.1 & 7.75 & 480 & 12.8 & 11.72 & 458 & 21.9 & 6.53 \\
\hline $\mathrm{Sm}$ & 453 & 11 & 459 & 1.76 & 1.38 & 483 & 29.6 & 6.70 & 504 & 12.9 & 11.31 & 480 & 26.2 & 6.03 \\
\hline $\mathrm{Eu}$ & 447 & 12 & 453 & 2.51 & 1.38 & 479 & 30.2 & 7.18 & 494 & 13.3 & 10.61 & 474 & 25.7 & 6.01 \\
\hline $\mathrm{Gd}$ & 449 & 12 & 455 & 2.23 & 1.37 & 478 & 29.3 & 6.38 & 513 & 18.5 & 14.24 & 479 & 30.5 & 6.71 \\
\hline $\mathrm{Tb}$ & 437 & 9 & 443 & 1.19 & 1.38 & 469 & 28.5 & 7.39 & 481 & 13.9 & 10.02 & 463 & 24.4 & 5.92 \\
\hline Dy & 437 & 11 & 443 & 1.92 & 1.39 & 471 & 26.2 & 7.87 & 483 & 16.3 & 10.61 & 464 & 24.6 & 6.27 \\
\hline Но & 449 & 12 & 455 & 1.56 & 1.39 & 473 & 33.2 & 5.25 & 494 & 15.6 & 9.94 & 472 & 26.4 & 5.13 \\
\hline $\mathrm{Er}$ & 455 & 14 & 461 & 1.64 & 1.38 & 474 & 30.9 & 4.19 & 517 & 16.7 & 13.52 & 481 & 30.5 & 5.72 \\
\hline $\mathrm{Tm}$ & 435 & 10 & 441 & 1.42 & 1.39 & 487 & 44.9 & 11.89 & 475 & 16.4 & 9.14 & 467 & 34.8 & 7.32 \\
\hline $\mathrm{Yb}$ & 450 & 9 & 456 & 4.16 & 1.39 & 500 & 44.3 & 11.13 & 503 & 14 & 11.68 & 485 & 35.2 & 7.74 \\
\hline $\mathrm{Lu}$ & 439 & 8 & 445 & 1.56 & 1.39 & 484 & 38.4 & 10.28 & 485 & 17.2 & 10.57 & 470 & 31.3 & 7.13 \\
\hline $\mathrm{Hf}$ & 435 & 12 & 441 & 1.78 & 1.37 & 460 & 20.7 & 5.83 & 466 & 18 & 7.07 & 455 & 18.9 & 4.55 \\
\hline $\mathrm{Ta}$ & 482 & 4 & 489 & 1.77 & 1.38 & 525 & 28.3 & 8.89 & 483 & 12 & 0.14 & 500 & 26.2 & 3.77 \\
\hline $\mathrm{Pb}$ & 426 & 1 & 432 & 1.76 & 1.38 & 421 & 14 & -1.20 & 448 & 15.8 & 5.25 & 432 & 16.1 & 1.50 \\
\hline Th & 457.2 & 1 & 464 & 1.94 & 1.39 & 487 & 17 & 6.51 & 510 & 18.1 & 11.44 & 485 & 23 & 6.00 \\
\hline $\mathrm{U}$ & 461.5 & 1 & 468 & 2.07 & 1.37 & 485 & 23.5 & 5.04 & 485 & 13.8 & 5.10 & 479 & 17.9 & 3.72 \\
\hline
\end{tabular}


Chapter 2.1 LA-ICP-MS Transient Signal Quantification of NIST, MPI-DING, USGS and CGSG Glass Reference Materials by Ratioing, Standardization, and Normalization (RSN)

Table $3 b$

Element concentrations of NIST612 obtained from RSN strategy with NIST 610, StHs6/80-G and GSD-1G as external reference materials. Values are in units of $\mu \mathrm{g} / \mathrm{g}$ except for major elements which are specified in weight percent $(\% \mathrm{~m} / \mathrm{m})$. Standard deviation $(1 \mathrm{~s})$ stems from 73 measurements.

\begin{tabular}{|c|c|c|c|c|c|c|c|c|c|c|c|c|c|c|}
\hline \multicolumn{15}{|l|}{ NIST612 } \\
\hline \multirow[b]{2}{*}{ Elements } & \multicolumn{2}{|c|}{ Reference value } & \multicolumn{2}{|l|}{ NIST610 } & \multicolumn{4}{|c|}{ StHs6/80-G } & \multicolumn{3}{|l|}{ GSD-1G } & \multicolumn{3}{|c|}{ Prefer value } \\
\hline & Value & Uncertainty & Average & $1 \mathrm{~s}$ & $\operatorname{RSD}(\%)$ & Average & $1 \mathrm{~s}$ & $\operatorname{RSD}(\%)$ & Average & $1 \mathrm{~s}$ & $\operatorname{RSD}(\%)$ & Average & $1 \mathrm{~s}$ & $\operatorname{RSD}(\%)$ \\
\hline $\mathrm{TiO} 2$ & 0.0073 & 0.0004 & 0.0068 & 0.0005 & -7.67 & 0.0077 & 0.0006 & 4.40 & 0.0073 & 0.0005 & -0.51 & 0.0075 & 0.0006 & 2.10 \\
\hline $\mathrm{Al} 2 \mathrm{O} 3$ & 2.03 & 0.04 & 2.04 & 0.0182 & 0.49 & 2.17 & 0.0345 & 6.77 & 2.1 & 0.0327 & 3.29 & 2.1 & 0.0608 & 3.53 \\
\hline $\mathrm{FeO}(\mathrm{t})$ & 0.0066 & 0.0003 & 0.0068 & 0.0015 & 3.51 & 0.0074 & 0.0017 & 12.15 & 0.0076 & 0.0019 & 16.18 & 0.0075 & 0.0018 & 14.04 \\
\hline $\mathrm{MnO}$ & 0.0050 & 0.0001 & 0.005 & 0.0001 & 0.55 & 0.0054 & 0.0002 & 8.86 & 0.0054 & 0.0001 & 8.41 & 0.0054 & 0.0002 & 8.65 \\
\hline $\mathrm{MgO}$ & 0.0113 & 0.0008 & 0.009 & 0.001 & -20.12 & 0.0108 & 0.0012 & -3.84 & 0.0107 & 0.0013 & -5.40 & 0.0108 & 0.0013 & -4.57 \\
\hline $\mathrm{CaO}$ & 11.9 & 0.1 & 11.8 & 0.143 & -0.82 & 12.1 & 0.306 & 1.74 & 12.1 & 0.497 & 2.10 & 12 & 0.373 & 0.96 \\
\hline $\mathrm{Na} 2 \mathrm{O}$ & 13.7 & 0.3 & 13.6 & 0.166 & -0.49 & 13.3 & 0.331 & -3.19 & 13.6 & 0.301 & -1.02 & 13.5 & 0.319 & -1.59 \\
\hline $\mathrm{K} 2 \mathrm{O}$ & 0.0075 & 0.0003 & 0.0068 & 0.0004 & -8.98 & 0.0062 & 0.0005 & -17.36 & 0.0062 & 0.0004 & -17.87 & 0.0062 & 0.0005 & -17.60 \\
\hline $\mathrm{P} 2 \mathrm{O} 5$ & 0.0107 & 0.0016 & 0.0121 & 0.0045 & 13.64 & 0.0134 & 0.0053 & 25.24 & 0.0108 & 0.0035 & 0.83 & 0.0122 & 0.0047 & 13.84 \\
\hline $\mathrm{Li}$ & 40.2 & 1.3 & 39.9 & 1.71 & -0.69 & 41.8 & 3.43 & 3.99 & 40.7 & 2.5 & 1.29 & 40.8 & 2.76 & 1.54 \\
\hline B & 34.3 & 1.7 & 35.8 & 3.98 & 4.46 & 36.7 & 8.64 & 7.14 & 28.7 & 2.88 & -16.29 & 34 & 6.8 & -0.93 \\
\hline $\mathrm{Sc}$ & 39.9 & 2.5 & 39.9 & 0.913 & -0.08 & 46 & 1.77 & 15.41 & 44.2 & 1.04 & 10.88 & 43.3 & 2.95 & 8.64 \\
\hline $\mathrm{V}$ & 38.8 & 1.2 & 39 & 0.5 & 0.44 & 42.7 & 1.08 & 10.09 & 41.2 & 0.718 & 6.17 & 40.9 & 1.76 & 5.54 \\
\hline $\mathrm{Cr}$ & 36.4 & 1.5 & 36.1 & 1.63 & -0.71 & 54.1 & 3.51 & 48.59 & 38 & 1.81 & 4.28 & 37 & 1.94 & 1.62 \\
\hline Co & 35.5 & 1 & 35.5 & 0.58 & 0.08 & 36.5 & 1.23 & 2.90 & 36.7 & 0.978 & 3.37 & 36.2 & 1.1 & 2.06 \\
\hline $\mathrm{Ni}$ & 38.8 & 0.2 & 38.8 & 1.46 & -0.10 & 46.5 & 3.06 & 19.77 & 39.5 & 1.51 & 1.91 & 39.1 & 1.53 & 0.84 \\
\hline $\mathrm{Cu}$ & 37.8 & 1.5 & 38.2 & 1.12 & 1.01 & 40.9 & 1.6 & 8.08 & 38.7 & 1.38 & 2.34 & 39.3 & 1.82 & 3.87 \\
\hline $\mathrm{Zn}$ & 39.1 & 1.7 & 39 & 3.19 & -0.23 & 39.6 & 4.14 & 1.29 & 37.8 & 3.96 & -3.44 & 38.8 & 3.85 & -0.68 \\
\hline $\mathrm{Ga}$ & 36.9 & 1.5 & 37 & 0.869 & 0.16 & 39 & 2.43 & 5.58 & 36.8 & 1.03 & -0.27 & 37.6 & 1.9 & 1.92 \\
\hline $\mathrm{Rb}$ & 31.4 & 0.4 & 32.1 & 0.409 & 2.19 & 32.7 & 1.2 & 4.13 & 30.9 & 0.794 & -1.48 & 31.9 & 1.13 & 1.75 \\
\hline $\mathrm{Sr}$ & 78.4 & 0.2 & 79.3 & 0.861 & 1.19 & 82.5 & 2.17 & 5.29 & 84.1 & 1.48 & 7.24 & 81.9 & 2.54 & 4.46 \\
\hline $\mathrm{Y}$ & 38.3 & 1.4 & 39.3 & 0.575 & 2.57 & 41.9 & 1.48 & 9.42 & 43.7 & 1.24 & 14.01 & 41.5 & 2.13 & 8.44 \\
\hline Mo & 37.4 & 1.5 & 37 & 0.791 & -1.03 & 48.4 & 7.14 & 29.49 & 38.7 & 1.44 & 3.47 & 37.8 & 1.42 & 1.07 \\
\hline $\mathrm{Sn}$ & 38.6 & 1.3 & 38.1 & 0.682 & -1.18 & 38.7 & 5.84 & 0.25 & 31.7 & 0.859 & -17.95 & 38.4 & 4.16 & -0.46 \\
\hline $\mathrm{Sb}$ & 34.7 & 1.8 & 34.4 & 0.631 & -0.99 & 29.4 & 7.56 & -15.29 & 39.5 & 1.39 & 13.80 & 34.4 & 0.631 & -0.99 \\
\hline $\mathrm{Cs}$ & 42.7 & 1.8 & 42.5 & 0.562 & -0.46 & 46.7 & 1.41 & 9.26 & 43 & 0.979 & 0.79 & 44.1 & 2.14 & 3.30 \\
\hline $\mathrm{Ba}$ & 39.3 & 0.9 & 39.9 & 0.835 & 1.53 & 40.8 & 1.27 & 3.85 & 40.5 & 1.41 & 3.14 & 40.4 & 1.25 & 2.82 \\
\hline $\mathrm{La}$ & 36 & 0.7 & 36.7 & 0.48 & 1.95 & 38.4 & 1.09 & 6.64 & 39.3 & 0.856 & 9.29 & 38.1 & 1.38 & 5.82 \\
\hline $\mathrm{Ce}$ & 38.4 & 0.7 & 39 & 0.519 & 1.48 & 41.2 & 1.03 & 7.33 & 41.3 & 0.856 & 7.48 & 40.5 & 1.36 & 5.34 \\
\hline $\operatorname{Pr}$ & 37.9 & 1 & 38.4 & 0.414 & 1.27 & 41.3 & 1.61 & 9.02 & 41 & 0.924 & 8.19 & 40.2 & 1.73 & 6.07 \\
\hline $\mathrm{Nd}$ & 35.5 & 0.7 & 35.8 & 0.77 & 0.73 & 38.5 & 1.28 & 8.37 & 39.3 & 1.2 & 10.73 & 37.8 & 1.87 & 6.43 \\
\hline $\mathrm{Sm}$ & 37.7 & 0.8 & 38.1 & 0.743 & 0.94 & 40.5 & 2.58 & 7.50 & 41.6 & 1.18 & 10.26 & 40 & 2.26 & 6.06 \\
\hline $\mathrm{Eu}$ & 35.6 & 0.8 & 36 & 0.443 & 1.15 & 38.1 & 2.69 & 6.98 & 39.2 & 1.01 & 10.01 & 37.7 & 2.14 & 5.88 \\
\hline $\mathrm{Gd}$ & 37.3 & 0.9 & 38.7 & 0.876 & 3.63 & 41.2 & 3.11 & 10.39 & 43.6 & 1.57 & 16.77 & 41 & 2.88 & 9.99 \\
\hline $\mathrm{Tb}$ & 37.6 & 1.1 & 37.8 & 0.542 & 0.47 & 41.3 & 1.87 & 9.97 & 41.2 & 1.18 & 9.45 & 40 & 2.12 & 6.51 \\
\hline Dy & 35.5 & 0.7 & 36.5 & 0.764 & 2.69 & 39.2 & 1.97 & 10.40 & 39.7 & 1.28 & 11.75 & 38.4 & 2.02 & 8.13 \\
\hline Ho & 38.3 & 0.8 & 38.7 & 0.514 & 1.10 & 40.7 & 1.68 & 6.16 & 42.2 & 1.38 & 10.19 & 40.5 & 1.91 & 5.63 \\
\hline $\mathrm{Er}$ & 38 & 0.9 & 39.4 & 0.82 & 3.57 & 41.2 & 1.81 & 8.35 & 44.2 & 1.65 & 16.26 & 41.5 & 2.46 & 9.10 \\
\hline $\mathrm{Tm}$ & 36.8 & 0.6 & 37.7 & 0.522 & 2.50 & 42.6 & 2.87 & 15.67 & 40.8 & 1.5 & 10.93 & 40.4 & 2.8 & 9.65 \\
\hline $\mathrm{Yb}$ & 39.2 & 0.9 & 39 & 0.788 & -0.39 & 43.7 & 3.65 & 11.59 & 43.1 & 1.42 & 10.01 & 41.9 & 3.15 & 6.94 \\
\hline $\mathrm{Lu}$ & 37 & 0.9 & 37.4 & 0.575 & 1.10 & 41.4 & 3.19 & 11.99 & 40.9 & 1.49 & 10.49 & 39.9 & 2.76 & 7.75 \\
\hline $\mathrm{Hf}$ & 36.7 & 1.2 & 37.9 & 1.33 & 3.34 & 40 & 1.69 & 8.99 & 40.1 & 1.61 & 9.31 & 39.3 & 1.85 & 7.13 \\
\hline $\mathrm{Ta}$ & 37.6 & 1.9 & 40.9 & 0.6 & 8.68 & 44.3 & 2.04 & 17.92 & 40.4 & 0.79 & 7.47 & 41.9 & 2.21 & 11.52 \\
\hline $\mathrm{Pb}$ & 38.6 & 0.2 & 39.3 & 0.796 & 2.01 & 38.7 & 1.6 & 0.44 & 40.2 & 1.22 & 4.30 & 39.4 & 1.39 & 2.16 \\
\hline Th & 37.8 & 0.08 & 38.4 & 0.616 & 1.53 & 41.2 & 1.1 & 8.94 & 42.3 & 1.34 & 11.83 & 40.5 & 1.94 & 7.24 \\
\hline $\mathrm{U}$ & 37.4 & 0.08 & 38.5 & 0.948 & 3.05 & 41 & 3.61 & 9.67 & 39.5 & 0.916 & 5.76 & 39.7 & 2.49 & 6.18 \\
\hline
\end{tabular}


Chapter 2.1 LA-ICP-MS Transient Signal Quantification of NIST, MPI-DING, USGS and CGSG Glass Reference Materials by Ratioing, Standardization, and Normalization (RSN)

\section{Table 3c}

Element concentrations of NIST614 obtained from RSN strategy with NIST 610, StHs6/80-G and GSD-1G as external reference materials. Values are in units of $\mu \mathrm{g} / \mathrm{g}$ except for major elements which are specified in weight percent $(\% \mathrm{~m} / \mathrm{m})$. Standard deviation $(1 \mathrm{~s})$ stems from $12 \mathrm{measurements}$.

\begin{tabular}{|c|c|c|c|c|c|c|c|c|c|c|c|c|c|c|}
\hline \multirow[t]{2}{*}{ NIST614 } & \multicolumn{2}{|c|}{ Reference value } & \multicolumn{2}{|l|}{ NIST610 } & \multirow[b]{2}{*}{$\operatorname{RSD}(\%)$} & \multicolumn{3}{|c|}{ StHs6/80-G } & \multicolumn{3}{|l|}{ GSD-1G } & \multicolumn{3}{|c|}{ Prefer value } \\
\hline & Value & Uncertainty & Average & $1 \mathrm{~s}$ & & Average & $1 \mathrm{~s}$ & $\operatorname{RSD}(\%)$ & Average & $1 \mathrm{~s}$ & $\operatorname{RSD}(\%)$ & Average & $1 \mathrm{~s}$ & $\operatorname{RSD}(\%)$ \\
\hline $\mathrm{TiO} 2$ & 0.0006 & 0.00004 & - & - & - & - & - & - & - & - & - & - & - & - \\
\hline $\mathrm{Al} 2 \mathrm{O} 3$ & 2.04 & 0.05 & 2.03 & 0.039 & -0.36 & 2.18 & 0.0226 & 6.99 & 2.14 & 0.0146 & 4.82 & 2.12 & 0.0713 & 3.73 \\
\hline $\mathrm{FeO}(\mathrm{t})$ & 0.0024 & 0.0008 & - & - & - & - & - & - & - & - & - & - & - & - \\
\hline $\mathrm{MnO}$ & 0.0002 & 0.00001 & - & - & - & - & - & - & - & - & - & - & - & - \\
\hline $\mathrm{MgO}$ & 0.0056 & 0.0003 & 0.0041 & 0.0003 & -27.53 & 0.0049 & 0.0004 & -12.90 & 0.0047 & 0.0004 & -15.33 & 0.0048 & 0.0004 & -13.95 \\
\hline $\mathrm{CaO}$ & 11.9 & 0.2 & 12.2 & 0.218 & 2.10 & 12.6 & 0.43 & 5.81 & 12.1 & 0.268 & 2.01 & 12.3 & 0.388 & 3.43 \\
\hline $\mathrm{Na} 2 \mathrm{O}$ & 13.7 & 0.3 & 13.6 & 0.058 & -0.99 & 13.1 & 0.282 & -4.64 & 13.4 & 0.331 & -2.13 & 13.3 & 0.328 & -2.63 \\
\hline $\mathrm{K} 2 \mathrm{O}$ & 0.0036 & 0.0001 & 0.0035 & 0.0007 & -3.31 & 0.0032 & 0.0007 & -11.66 & 0.0028 & 0.0006 & -22.64 & 0.003 & 0.0007 & -16.37 \\
\hline $\mathrm{P} 2 \mathrm{O} 5$ & 0.0026 & 0.0009 & - & - & - & - & - & - & - & - & - & - & - & - \\
\hline $\mathrm{Li}$ & 1.69 & 0.09 & 1.9 & 0.731 & 12.52 & 2 & 0.868 & 18.44 & 1.94 & 0.921 & 14.53 & 1.95 & 0.838 & 15.22 \\
\hline $\mathrm{Be}$ & 0.753 & 0.051 & - & - & - & - & - & - & - & - & - & - & - & - \\
\hline B & 1.49 & 0.19 & - & - & - & - & - & - & - & - & - & - & - & - \\
\hline $\mathrm{Sc}$ & 0.74 & - & 2.41 & 0.386 & 225.90 & 2.57 & 0.367 & 246.85 & 2.83 & 0.232 & 281.83 & 2.58 & 0.38 & 248.77 \\
\hline $\mathrm{V}$ & 1.01 & 0.04 & 1.05 & 0.0729 & 3.47 & 1.14 & 0.0939 & 13.04 & 1.11 & 0.0833 & 10.34 & 1.1 & 0.0939 & 8.82 \\
\hline $\mathrm{Cr}$ & 1.19 & 0.12 & - & - & - & - & - & - & - & - & - & - & - & - \\
\hline Co & 0.79 & 0.09 & 0.749 & 0.0572 & -5.19 & 0.767 & 0.0597 & -2.90 & 0.822 & 0.0706 & 4.02 & 0.775 & 0.0686 & -1.85 \\
\hline $\mathrm{Ni}$ & 1.1 & 0.1 & - & - & - & - & - & - & - & - & - & - & - & - \\
\hline $\mathrm{Cu}$ & 1.37 & 0.07 & 1.59 & 0.792 & 15.69 & 1.69 & 0.842 & 22.99 & 2.09 & 0.387 & 52.39 & 1.76 & 0.755 & 28.36 \\
\hline $\mathrm{Zn}$ & 2.79 & 0.38 & - & - & - & - & - & - & - & - & - & - & - & - \\
\hline $\mathrm{Ga}$ & 1.31 & 0.09 & 1.16 & 0.538 & -11.39 & 1.3 & 0.681 & -0.89 & 1.1 & 0.616 & -16.37 & 1.19 & 0.62 & -8.93 \\
\hline $\mathrm{Rb}$ & 0.855 & 0.005 & 0.95 & 0.0932 & 11.11 & 0.953 & 0.108 & 11.40 & 0.908 & 0.102 & 6.17 & 0.939 & 0.103 & 9.87 \\
\hline $\mathrm{Sr}$ & 45.8 & 0.1 & 47.7 & 1.44 & 4.22 & 48.5 & 1.48 & 5.82 & 50.8 & 1.15 & 10.86 & 48.8 & 1.85 & 6.61 \\
\hline $\mathrm{Y}$ & 0.79 & 0.032 & 0.837 & 0.0663 & 5.89 & 0.86 & 0.0668 & 8.84 & 0.903 & 0.0772 & 14.26 & 0.863 & 0.0744 & 9.24 \\
\hline Mo & 0.8 & 0.03 & 0.827 & 0.0772 & 3.33 & 1.18 & 0.246 & 47.50 & 0.894 & 0.11 & 11.81 & 0.856 & 0.0987 & 6.96 \\
\hline $\mathrm{Sn}$ & 1.68 & 0.15 & 1.54 & 0.141 & -8.28 & 1.46 & 0.298 & -13.14 & 1.26 & 0.097 & -25.13 & 1.5 & 0.237 & -10.71 \\
\hline $\mathrm{Sb}$ & 0.79 & 0.064 & 0.72 & 0.076 & -8.86 & 0.443 & 0.17 & -43.93 & 0.807 & 0.112 & 2.11 & 0.72 & 0.076 & -8.86 \\
\hline $\mathrm{Cs}$ & 0.664 & 0.034 & 0.734 & 0.0513 & 10.53 & 0.804 & 0.0518 & 21.03 & 0.729 & 0.0663 & 9.82 & 0.758 & 0.0658 & 14.16 \\
\hline $\mathrm{Ba}$ & 3.2 & 0.09 & 3.52 & 0.479 & 9.97 & 3.54 & 0.445 & 10.65 & 3.44 & 0.318 & 7.50 & 3.51 & 0.43 & 9.55 \\
\hline $\mathrm{La}$ & 0.72 & 0.013 & 0.749 & 0.0601 & 4.04 & 0.761 & 0.0463 & 5.66 & 0.793 & 0.041 & 10.19 & 0.765 & 0.0536 & 6.30 \\
\hline $\mathrm{Ce}$ & 0.813 & 0.025 & 0.807 & 0.0693 & -0.71 & 0.845 & 0.0625 & 3.92 & 0.852 & 0.0574 & 4.74 & 0.833 & 0.0667 & 2.46 \\
\hline $\operatorname{Pr}$ & 0.768 & 0.015 & 0.796 & 0.0521 & 3.58 & 0.837 & 0.0544 & 9.04 & 0.841 & 0.0595 & 9.51 & 0.823 & 0.0589 & 7.18 \\
\hline $\mathrm{Nd}$ & 0.752 & 0.014 & 0.727 & 0.103 & -3.37 & 0.758 & 0.105 & 0.73 & 0.798 & 0.122 & 6.09 & 0.757 & 0.113 & 0.70 \\
\hline $\mathrm{Sm}$ & 0.754 & 0.013 & 0.773 & 0.108 & 2.45 & 0.815 & 0.121 & 8.09 & 0.822 & 0.13 & 9.05 & 0.802 & 0.121 & 6.30 \\
\hline $\mathrm{Eu}$ & 0.77 & 0.016 & 0.813 & 0.0498 & 5.64 & 0.865 & 0.0839 & 12.27 & 0.89 & 0.0514 & 15.60 & 0.853 & 0.0719 & 10.77 \\
\hline $\mathrm{Gd}$ & 0.763 & 0.021 & 0.807 & 0.108 & 5.72 & 0.891 & 0.112 & 16.75 & 0.847 & 0.0936 & 10.97 & 0.848 & 0.112 & 11.16 \\
\hline $\mathrm{Tb}$ & 0.739 & 0.02 & 0.785 & 0.0528 & 6.24 & 0.868 & 0.0577 & 17.40 & 0.844 & 0.0506 & 14.21 & 0.831 & 0.065 & 12.47 \\
\hline Dy & 0.746 & 0.022 & 0.782 & 0.0969 & 4.78 & 0.816 & 0.102 & 9.36 & 0.846 & 0.118 & 13.35 & 0.812 & 0.108 & 8.78 \\
\hline Ho & 0.749 & 0.015 & 0.802 & 0.044 & 7.05 & 0.794 & 0.0445 & 6.05 & 0.843 & 0.0497 & 12.58 & 0.81 & 0.0501 & 8.20 \\
\hline $\mathrm{Er}$ & 0.74 & 0.017 & 0.757 & 0.0714 & 2.25 & 0.776 & 0.0696 & 4.82 & 0.833 & 0.0736 & 12.61 & 0.784 & 0.0778 & 6.01 \\
\hline $\mathrm{Tm}$ & 0.732 & 0.02 & 0.742 & 0.0216 & 1.31 & 0.777 & 0.0282 & 6.12 & 0.776 & 0.0306 & 6.00 & 0.764 & 0.0315 & 4.34 \\
\hline $\mathrm{Yb}$ & 0.777 & 0.021 & 0.738 & 0.0831 & -4.98 & 0.739 & 0.0673 & -4.87 & 0.832 & 0.0716 & 7.11 & 0.764 & 0.0854 & -1.64 \\
\hline $\mathrm{Lu}$ & 0.732 & 0.018 & 0.761 & 0.0515 & 3.98 & 0.864 & 0.101 & 18.01 & 0.821 & 0.0451 & 12.14 & 0.815 & 0.0847 & 11.31 \\
\hline $\mathrm{Hf}$ & 0.711 & 0.022 & 0.765 & 0.0877 & 7.59 & 0.783 & 0.098 & 10.17 & 0.831 & 0.0844 & 16.89 & 0.79 & 0.0945 & 11.07 \\
\hline $\mathrm{Ta}$ & 0.808 & 0.026 & 0.824 & 0.0576 & 2.03 & 0.907 & 0.0708 & 12.28 & 0.828 & 0.0305 & 2.53 & 0.856 & 0.0693 & 5.90 \\
\hline $\mathrm{Pb}$ & 2.32 & 0.04 & 2.43 & 0.107 & 4.71 & 2.32 & 0.166 & -0.22 & 2.57 & 0.29 & 10.92 & 2.43 & 0.218 & 4.61 \\
\hline Th & 0.748 & 0.006 & 0.8 & 0.0582 & 6.99 & 0.842 & 0.0811 & 12.52 & 0.874 & 0.0606 & 16.84 & 0.835 & 0.0741 & 11.69 \\
\hline $\mathrm{U}$ & 0.823 & 0.002 & 0.858 & 0.0459 & 4.23 & 0.871 & 0.0332 & 5.86 & 0.874 & 0.0389 & 6.25 & 0.867 & 0.0404 & 5.38 \\
\hline
\end{tabular}


Chapter 2.1 LA-ICP-MS Transient Signal Quantification of NIST, MPI-DING, USGS and CGSG Glass Reference Materials by Ratioing, Standardization, and Normalization (RSN)

\section{Table $3 \mathrm{~d}$}

Element concentrations of ATHO-G obtained from RSN strategy with NIST 610, StHs6/80-G and GSD-1G as external reference materials. Values are in units of $\mu \mathrm{g} / \mathrm{g}$ except for major elements which are specified in weight percent $(\% \mathrm{~m} / \mathrm{m})$. Standard deviation $(1 \mathrm{~s})$ stems from 78 measurements.

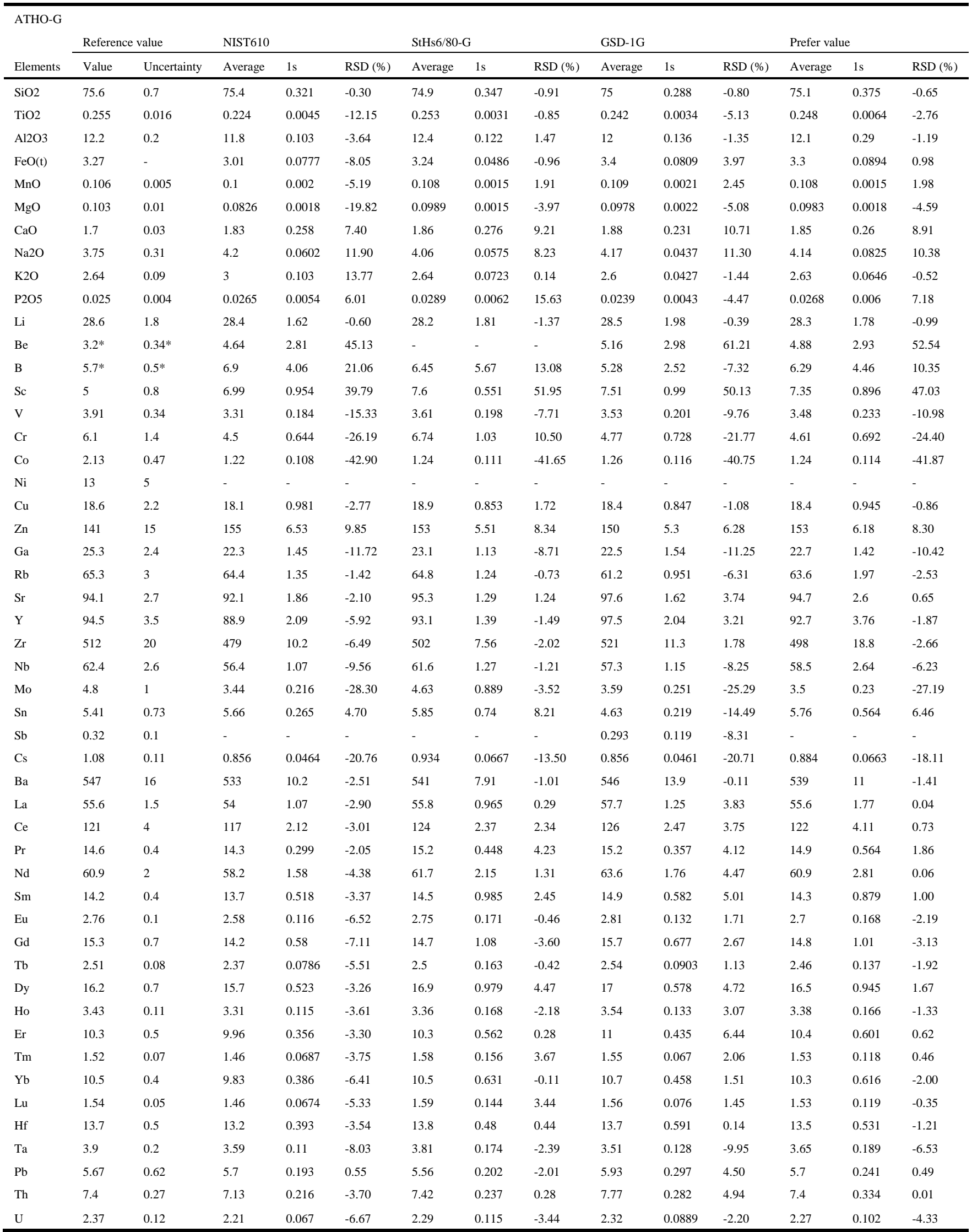


Chapter 2.1 LA-ICP-MS Transient Signal Quantification of NIST, MPI-DING, USGS and CGSG Glass Reference Materials by Ratioing, Standardization, and Normalization (RSN)

\section{Table $3 \mathrm{e}$}

Element concentrations of KL2-G obtained from RSN strategy with NIST 610, StHs6/80-G and GSD-1G as external reference materials. Values are in units of $\mu \mathrm{g} / \mathrm{g}$ except for major elements which are specified in weight percent $(\% \mathrm{~m} / \mathrm{m})$. Standard deviation $(1 \mathrm{~s})$ stems from 78 measurements.

\begin{tabular}{|c|c|c|c|c|c|c|c|c|c|c|c|c|c|c|}
\hline KL2-G & Reference value & value & NIST610 & & \multicolumn{4}{|c|}{ StHs6/80-G } & \multicolumn{3}{|l|}{ GSD-1G } & \multicolumn{3}{|c|}{ Prefer value } \\
\hline Elements & Value & Uncertainty & Average & $1 \mathrm{~s}$ & $\operatorname{RSD}(\%)$ & Average & $1 \mathrm{~s}$ & $\operatorname{RSD}(\%)$ & Average & $1 \mathrm{~s}$ & $\operatorname{RSD}(\%)$ & Average & $1 \mathrm{~s}$ & $\operatorname{RSD}(\%)$ \\
\hline $\mathrm{TiO} 2$ & 2.56 & 0.09 & 2.4 & 0.0566 & -6.22 & 2.62 & 0.0411 & 2.48 & 2.51 & 0.0222 & -1.97 & 2.57 & 0.066 & 0.54 \\
\hline $\mathrm{Al} 2 \mathrm{O} 3$ & 13.3 & 0.2 & 13 & 0.182 & -2.01 & 13.3 & 0.127 & 0.37 & 12.9 & 0.178 & -2.94 & 13.1 & 0.249 & -1.43 \\
\hline $\mathrm{FeO}(\mathrm{t})$ & 10.7 & 0.1 & 10.5 & 0.243 & -2.14 & 11 & 0.149 & 2.57 & 11.5 & 0.243 & 7.28 & 11.2 & 0.292 & 4.48 \\
\hline $\mathrm{MnO}$ & 0.165 & 0.009 & 0.168 & 0.0033 & 1.69 & 0.175 & 0.002 & 6.14 & 0.176 & 0.0023 & 6.41 & 0.175 & 0.002 & 6.18 \\
\hline $\mathrm{MgO}$ & 7.34 & 0.09 & 6.39 & 0.103 & -12.88 & 7.43 & 0.0739 & 1.24 & 7.34 & 0.137 & -0.03 & 7.39 & 0.117 & 0.63 \\
\hline $\mathrm{CaO}$ & 10.9 & 0.2 & 10.9 & 0.251 & 0.39 & 10.8 & 0.167 & -0.94 & 10.9 & 0.309 & -0.39 & 10.9 & 0.253 & -0.29 \\
\hline $\mathrm{Na} 2 \mathrm{O}$ & 2.35 & 0.08 & 2.38 & 0.0681 & 1.44 & 2.24 & 0.0742 & -4.58 & 2.31 & 0.0594 & -1.91 & 2.31 & 0.0913 & -1.66 \\
\hline $\mathrm{K} 2 \mathrm{O}$ & 0.48 & 0.01 & 0.566 & 0.0298 & 17.86 & 0.485 & 0.0164 & 1.13 & 0.477 & 0.0172 & -0.56 & 0.483 & 0.0168 & 0.54 \\
\hline $\mathrm{P} 2 \mathrm{O} 5$ & 0.232 & 0.026 & 0.251 & 0.0212 & 8.17 & 0.268 & 0.0172 & 15.51 & 0.236 & 0.0082 & 1.64 & 0.254 & 0.0212 & 9.54 \\
\hline $\mathrm{Li}$ & 5.1 & 0.5 & 5.83 & 1.18 & 14.28 & 5.65 & 1.09 & 10.71 & 5.67 & 1.31 & 11.16 & 5.69 & 1.19 & 11.55 \\
\hline B & $2.73^{*}$ & $0.28^{*}$ & - & - & - & - & - & - & - & - & - & - & - & - \\
\hline $\mathrm{Sc}$ & 31.8 & 0.9 & 31.4 & 1.07 & -1.12 & 33.5 & 1.95 & 5.23 & 33.2 & 0.744 & 4.36 & 32.6 & 1.66 & 2.63 \\
\hline $\mathrm{V}$ & 309 & 38 & 323 & 6.1 & 4.51 & 341 & 5.35 & 10.33 & 333 & 5.31 & 7.64 & 332 & 9.51 & 7.49 \\
\hline $\mathrm{Cr}$ & 294 & 27 & 288 & 8.99 & -1.89 & 423 & 21.6 & 43.88 & 296 & 10.6 & 0.77 & 291 & 9.37 & -0.97 \\
\hline Co & 41.2 & 2.3 & 45.6 & 1.15 & 10.61 & 45.1 & 1.3 & 9.49 & 46.1 & 1.06 & 11.83 & 45.5 & 1.21 & 10.46 \\
\hline $\mathrm{Ni}$ & 112 & 5 & 120 & 3.9 & 7.20 & 138 & 7.17 & 23.05 & 119 & 3.46 & 6.29 & 120 & 3.74 & 6.71 \\
\hline $\mathrm{Cu}$ & 87.9 & 9.1 & 98.4 & 2.62 & 11.91 & 100 & 2.16 & 14.08 & 96.7 & 3.11 & 9.99 & 98.5 & 2.97 & 12.03 \\
\hline $\mathrm{Zn}$ & 110 & 10 & 125 & 6.22 & 13.41 & 121 & 4.88 & 9.98 & 118 & 3.61 & 7.14 & 121 & 5.85 & 10.40 \\
\hline $\mathrm{Ga}$ & 20 & 1.2 & 22.5 & 0.775 & 12.50 & 22.7 & 1.52 & 13.67 & 21.9 & 0.956 & 9.48 & 22.4 & 1.19 & 12.01 \\
\hline $\mathrm{Rb}$ & 8.7 & 0.4 & 9.44 & 0.283 & 8.46 & 9.25 & 0.3 & 6.31 & 8.72 & 0.263 & 0.20 & 9.17 & 0.404 & 5.40 \\
\hline $\mathrm{Sr}$ & 356 & 8 & 353 & 8.42 & -0.90 & 353 & 4.82 & -0.71 & 362 & 4.7 & 1.78 & 355 & 7.27 & -0.14 \\
\hline $\mathrm{Y}$ & 25.4 & 1.1 & 24.1 & 0.798 & -4.97 & 24.5 & 0.646 & -3.46 & 25.7 & 0.578 & 1.25 & 24.7 & 0.923 & -2.75 \\
\hline Mo & 3.6 & 0.6 & 3.33 & 0.244 & -7.59 & 4.23 & 0.632 & 17.59 & 3.37 & 0.238 & -6.28 & 3.33 & 0.23 & -7.39 \\
\hline Sn & 1.54 & 0.29 & 1.51 & 0.149 & -1.91 & 1.59 & 0.308 & 3.26 & 1.2 & 0.128 & -22.05 & 1.55 & 0.245 & 0.67 \\
\hline $\mathrm{Sb}$ & 0.14 & 0.03 & - & - & - & 0.638 & 4.73 & 355.99 & - & - & - & - & - & - \\
\hline $\mathrm{Cs}$ & 0.115 & 0.009 & 0.125 & 0.0343 & 8.41 & 0.132 & 0.0373 & 14.72 & - & - & - & 0.127 & 0.0357 & 10.66 \\
\hline $\mathrm{Ba}$ & 123 & 5 & 123 & 2.74 & -0.18 & 121 & 2.2 & -1.46 & 123 & 3.01 & -0.18 & 122 & 2.71 & -0.69 \\
\hline $\mathrm{La}$ & 13.1 & 0.2 & 13.1 & 0.327 & -0.26 & 13.1 & 0.278 & -0.02 & 13.5 & 0.277 & 3.31 & 13.2 & 0.356 & 0.80 \\
\hline $\mathrm{Ce}$ & 32.4 & 0.7 & 32.3 & 0.681 & -0.30 & 33.1 & 0.635 & 2.06 & 33.4 & 0.626 & 3.06 & 32.9 & 0.794 & 1.48 \\
\hline $\operatorname{Pr}$ & 4.6 & 0.1 & 4.47 & 0.147 & -2.78 & 4.62 & 0.132 & 0.42 & 4.6 & 0.126 & 0.02 & 4.56 & 0.149 & -0.89 \\
\hline $\mathrm{Nd}$ & 21.6 & 0.4 & 20.9 & 0.85 & -3.11 & 21.6 & 0.984 & 0.09 & 22.1 & 0.832 & 2.38 & 21.5 & 1.01 & -0.48 \\
\hline $\mathrm{Sm}$ & 5.54 & 0.09 & 5.49 & 0.288 & -0.96 & 5.66 & 0.41 & 2.18 & 5.78 & 0.298 & 4.40 & 5.63 & 0.358 & 1.66 \\
\hline $\mathrm{Eu}$ & 1.92 & 0.04 & 1.89 & 0.0967 & -1.62 & 1.94 & 0.139 & 1.26 & 1.99 & 0.0901 & 3.83 & 1.94 & 0.12 & 0.95 \\
\hline $\mathrm{Gd}$ & 5.92 & 0.2 & 5.77 & 0.329 & -2.61 & 5.8 & 0.435 & -2.08 & 6.23 & 0.345 & 5.20 & 5.91 & 0.424 & -0.25 \\
\hline $\mathrm{Tb}$ & 0.89 & 0.031 & 0.848 & 0.0432 & -4.67 & 0.873 & 0.06 & -1.92 & 0.878 & 0.0421 & -1.30 & 0.866 & 0.0512 & -2.74 \\
\hline Dy & 5.22 & 0.12 & 5.06 & 0.226 & -3.14 & 5.32 & 0.4 & 1.95 & 5.26 & 0.206 & 0.82 & 5.21 & 0.318 & -0.26 \\
\hline Ho & 0.961 & 0.022 & 0.945 & 0.0561 & -1.71 & 0.934 & 0.065 & -2.83 & 0.979 & 0.0554 & 1.86 & 0.949 & 0.0616 & -1.22 \\
\hline $\mathrm{Er}$ & 2.54 & 0.07 & 2.5 & 0.129 & -1.53 & 2.51 & 0.156 & -0.99 & 2.66 & 0.139 & 4.74 & 2.55 & 0.159 & 0.46 \\
\hline $\mathrm{Tm}$ & 0.331 & 0.009 & 0.323 & 0.0277 & -2.39 & 0.336 & 0.0387 & 1.38 & 0.333 & 0.0286 & 0.67 & 0.33 & 0.0328 & -0.30 \\
\hline $\mathrm{Yb}$ & 2.1 & 0.05 & 1.99 & 0.16 & -5.23 & 2.07 & 0.218 & -1.41 & 2.1 & 0.176 & 0.00 & 2.05 & 0.194 & -2.33 \\
\hline $\mathrm{Lu}$ & 0.285 & 0.009 & 0.278 & 0.0254 & -2.49 & 0.293 & 0.0346 & 2.71 & 0.289 & 0.025 & 1.24 & 0.286 & 0.0297 & 0.40 \\
\hline $\mathrm{Hf}$ & 3.93 & 0.14 & 3.94 & 0.241 & 0.15 & 4.01 & 0.293 & 1.99 & 3.98 & 0.278 & 1.21 & 3.97 & 0.267 & 0.93 \\
\hline $\mathrm{Ta}$ & 0.961 & 0.022 & 0.922 & 0.0513 & -4.11 & 0.949 & 0.057 & -1.26 & 0.87 & 0.0496 & -9.43 & 0.918 & 0.0607 & -4.47 \\
\hline $\mathrm{Pb}$ & 2.07 & 0.1 & 2.09 & 0.125 & 1.04 & 1.98 & 0.115 & -4.14 & 2.11 & 0.147 & 1.96 & 2.05 & 0.127 & -0.89 \\
\hline Th & 1.02 & 0.03 & 0.997 & 0.0623 & -2.25 & 1.01 & 0.0707 & -0.70 & 1.06 & 0.0744 & 3.45 & 1.02 & 0.0683 & -0.37 \\
\hline $\mathrm{U}$ & 0.548 & 0.016 & 0.543 & 0.045 & -0.92 & 0.547 & 0.0496 & -0.17 & 0.547 & 0.0452 & -0.21 & 0.545 & 0.0466 & -0.61 \\
\hline
\end{tabular}


Chapter 2.1 LA-ICP-MS Transient Signal Quantification of NIST, MPI-DING, USGS and CGSG Glass Reference Materials by Ratioing, Standardization, and Normalization (RSN)

\section{Table 3f}

Element concentrations of ML3B-G obtained from RSN strategy with NIST 610, StHs6/80-G and GSD-1G as external reference materials. Values are in units of $\mu \mathrm{g} / \mathrm{g}$ except for major elements which are specified in weight percent $(\% \mathrm{~m} / \mathrm{m})$. Standard deviation $(1 \mathrm{~s})$ stems from $78 \mathrm{measurements}$.

\begin{tabular}{|c|c|c|c|c|c|c|c|c|c|c|c|c|c|c|}
\hline \multirow[t]{2}{*}{ ML3B-G } & \multicolumn{2}{|c|}{ Reference value } & \multicolumn{2}{|l|}{ NIST610 } & \multirow[b]{2}{*}{$\operatorname{RSD}(\%)$} & \multicolumn{3}{|c|}{ StHs6/80-G } & \multicolumn{3}{|l|}{ GSD-1G } & \multicolumn{3}{|c|}{ Prefer value } \\
\hline & Value & Uncertainty & Average & $1 \mathrm{~s}$ & & Average & $1 \mathrm{~s}$ & $\operatorname{RSD}(\%)$ & Average & $1 \mathrm{~s}$ & $\operatorname{RSD}(\%)$ & Average & $1 \mathrm{~s}$ & $\operatorname{RSD}(\%)$ \\
\hline $\mathrm{TiO} 2$ & 2.13 & 0.09 & 1.95 & 0.0462 & -8.61 & 2.13 & 0.039 & -0.11 & 2.03 & 0.0288 & -4.47 & 2.09 & 0.0554 & -1.92 \\
\hline $\mathrm{Al} 2 \mathrm{O} 3$ & 13.6 & 0.2 & 13.3 & 0.275 & -1.92 & 13.7 & 0.203 & 0.83 & 13.2 & 0.226 & -2.61 & 13.4 & 0.315 & -1.14 \\
\hline $\mathrm{FeO}(\mathrm{t})$ & 10.9 & 0.1 & 10.7 & 0.284 & -1.42 & 11.2 & 0.145 & 2.81 & 11.7 & 0.205 & 7.44 & 11.4 & 0.297 & 4.76 \\
\hline $\mathrm{MnO}$ & 0.17 & 0.009 & 0.171 & 0.0036 & 0.46 & 0.179 & 0.0022 & 5.09 & 0.179 & 0.0023 & 5.03 & 0.179 & 0.0023 & 5.06 \\
\hline $\mathrm{MgO}$ & 6.59 & 0.08 & 5.75 & 0.0953 & -12.82 & 6.69 & 0.0752 & 1.48 & 6.6 & 0.128 & 0.08 & 6.64 & 0.113 & 0.81 \\
\hline $\mathrm{CaO}$ & 10.5 & 0.1 & 10.3 & 0.212 & -1.60 & 10.2 & 0.154 & -2.57 & 10.3 & 0.333 & -1.89 & 10.3 & 0.24 & -1.97 \\
\hline $\mathrm{Na} 2 \mathrm{O}$ & 2.4 & 0.06 & 2.44 & 0.0748 & 1.68 & 2.29 & 0.0413 & -4.51 & 2.36 & 0.0592 & -1.66 & 2.36 & 0.0875 & -1.50 \\
\hline $\mathrm{K} 2 \mathrm{O}$ & 0.385 & 0.004 & 0.444 & 0.022 & 15.20 & 0.383 & 0.0125 & -0.65 & 0.375 & 0.0131 & -2.47 & 0.38 & 0.0126 & -1.27 \\
\hline $\mathrm{P} 2 \mathrm{O} 5$ & 0.23 & 0.025 & 0.222 & 0.0185 & -3.67 & 0.238 & 0.0147 & 3.37 & 0.209 & 0.0085 & -9.35 & 0.225 & 0.0189 & -2.06 \\
\hline $\mathrm{Li}$ & 4.5 & 0.4 & 4.57 & 1.41 & 1.62 & 4.46 & 1.36 & -0.96 & 4.32 & 1.41 & -4.02 & 4.46 & 1.35 & -0.91 \\
\hline B & $2.5^{*}$ & $0.6^{*}$ & - & - & - & - & - & - & - & - & - & - & - & - \\
\hline $\mathrm{Sc}$ & 31.6 & 1.6 & 29.7 & 0.925 & -5.94 & 31.9 & 1.69 & 0.87 & 31.6 & 0.657 & -0.09 & 31 & 1.56 & -1.84 \\
\hline $\mathrm{V}$ & 268 & 23 & 292 & 5.79 & 9.07 & 309 & 6.05 & 15.41 & 302 & 4.78 & 12.61 & 301 & 9.11 & 12.40 \\
\hline $\mathrm{Cr}$ & 177 & 23 & 159 & 5.05 & -10.14 & 231 & 13.6 & 30.39 & 164 & 6.34 & -7.60 & 161 & 6.08 & -9.09 \\
\hline Co & 41.2 & 3.5 & 45.4 & 1.13 & 10.10 & 44.9 & 1.32 & 9.06 & 45.8 & 1.03 & 11.26 & 45.3 & 1.22 & 9.99 \\
\hline $\mathrm{Ni}$ & 107 & 9 & 115 & 3.21 & 7.27 & 133 & 6.91 & 24.07 & 114 & 2.94 & 6.59 & 114 & 3.09 & 6.96 \\
\hline $\mathrm{Cu}$ & 112 & 10 & 127 & 3.29 & 13.09 & 130 & 3.28 & 15.91 & 124 & 4.13 & 11.08 & 127 & 4.14 & 13.42 \\
\hline $\mathrm{Zn}$ & 108 & 14 & 125 & 6.84 & 15.39 & 121 & 4.89 & 12.44 & 118 & 4.4 & 9.70 & 122 & 6.03 & 12.80 \\
\hline $\mathrm{Ga}$ & 19.6 & 2.1 & 21.1 & 0.962 & 7.53 & 21.4 & 1.44 & 9.15 & 20.2 & 0.83 & 2.96 & 20.9 & 1.22 & 6.75 \\
\hline $\mathrm{Rb}$ & 5.8 & 0.21 & 6.37 & 0.257 & 9.76 & 6.27 & 0.245 & 8.04 & 5.9 & 0.242 & 1.69 & 6.2 & 0.31 & 6.93 \\
\hline $\mathrm{Sr}$ & 312 & 4 & 308 & 7.66 & -1.39 & 307 & 5.79 & -1.50 & 316 & 4.79 & 1.23 & 310 & 7.35 & -0.70 \\
\hline $\mathrm{Y}$ & 23.9 & 0.7 & 22.7 & 0.793 & -5.01 & 23.1 & 0.575 & -3.32 & 24.3 & 0.566 & 1.54 & 23.3 & 0.915 & -2.58 \\
\hline Mo & 16.7 & 2.3 & 18.5 & 1.95 & 10.96 & 23.5 & 4.28 & 40.51 & 18.7 & 1.99 & 11.83 & 18.5 & 1.95 & 10.93 \\
\hline $\mathrm{Sn}$ & 1.14 & 0.33 & 1.15 & 0.136 & 1.10 & 1.23 & 0.27 & 7.49 & 0.918 & 0.11 & -19.51 & 1.19 & 0.217 & 4.30 \\
\hline $\mathrm{Sb}$ & $0.11^{*}$ & $0.05^{*}$ & - & - & - & - & - & - & - & - & - & - & - & - \\
\hline Cs & 0.14 & 0.012 & 0.157 & 0.0337 & 11.92 & 0.165 & 0.0365 & 18.04 & 0.154 & 0.0369 & 9.80 & 0.159 & 0.0359 & 13.83 \\
\hline $\mathrm{Ba}$ & 80.1 & 2.2 & 79.6 & 2.02 & -0.67 & 78.8 & 1.86 & -1.64 & 79.2 & 2 & -1.14 & 79.2 & 2 & -1.17 \\
\hline $\mathrm{La}$ & 8.99 & 0.13 & 8.78 & 0.252 & -2.39 & 8.83 & 0.178 & -1.81 & 9.13 & 0.171 & 1.52 & 8.89 & 0.254 & -1.10 \\
\hline $\mathrm{Ce}$ & 23.1 & 0.3 & 22.7 & 0.569 & -1.67 & 23.3 & 0.488 & 0.86 & 23.5 & 0.467 & 1.73 & 23.2 & 0.614 & 0.24 \\
\hline $\operatorname{Pr}$ & 3.43 & 0.06 & 3.3 & 0.118 & -3.90 & 3.39 & 0.118 & -1.25 & 3.39 & 0.109 & -1.17 & 3.35 & 0.124 & -2.21 \\
\hline $\mathrm{Nd}$ & 16.7 & 0.2 & 16.1 & 0.736 & -3.88 & 16.6 & 0.764 & -0.47 & 17.1 & 0.753 & 2.10 & 16.5 & 0.84 & -1.04 \\
\hline $\mathrm{Sm}$ & 4.75 & 0.07 & 4.57 & 0.3 & -3.87 & 4.78 & 0.382 & 0.72 & 4.79 & 0.303 & 0.83 & 4.71 & 0.349 & -0.90 \\
\hline $\mathrm{Eu}$ & 1.67 & 0.02 & 1.65 & 0.104 & -1.44 & 1.69 & 0.115 & 1.01 & 1.74 & 0.109 & 4.29 & 1.69 & 0.117 & 1.11 \\
\hline $\mathrm{Gd}$ & 5.26 & 0.23 & 5.1 & 0.312 & -2.99 & 5.18 & 0.392 & -1.50 & 5.52 & 0.301 & 4.92 & 5.25 & 0.385 & -0.17 \\
\hline $\mathrm{Tb}$ & 0.797 & 0.021 & 0.771 & 0.0443 & -3.24 & 0.796 & 0.0512 & -0.10 & 0.807 & 0.0471 & 1.21 & 0.79 & 0.0495 & -0.88 \\
\hline Dy & 4.84 & 0.07 & 4.66 & 0.281 & -3.80 & 4.86 & 0.377 & 0.36 & 4.87 & 0.267 & 0.52 & 4.79 & 0.332 & -1.09 \\
\hline Ho & 0.906 & 0.018 & 0.887 & 0.0549 & -2.07 & 0.883 & 0.0697 & -2.55 & 0.923 & 0.0551 & 1.82 & 0.896 & 0.0632 & -1.14 \\
\hline Er & 2.44 & 0.05 & 2.38 & 0.14 & -2.40 & 2.39 & 0.162 & -2.00 & 2.55 & 0.143 & 4.43 & 2.43 & 0.167 & -0.34 \\
\hline $\mathrm{Tm}$ & 0.324 & 0.007 & 0.309 & 0.0291 & -4.68 & 0.331 & 0.0332 & 2.04 & 0.318 & 0.0293 & -2.01 & 0.319 & 0.0321 & -1.47 \\
\hline $\mathrm{Yb}$ & 2.06 & 0.04 & 1.93 & 0.172 & -6.35 & 2.02 & 0.235 & -1.79 & 2.04 & 0.188 & -0.94 & 1.99 & 0.207 & -3.16 \\
\hline $\mathrm{Lu}$ & 0.286 & 0.006 & 0.272 & 0.0274 & -4.87 & 0.286 & 0.0377 & -0.08 & 0.286 & 0.0251 & 0.07 & 0.281 & 0.0316 & -1.65 \\
\hline $\mathrm{Hf}$ & 3.22 & 0.08 & 3.19 & 0.212 & -0.87 & 3.26 & 0.229 & 1.16 & 3.24 & 0.235 & 0.58 & 3.23 & 0.225 & 0.30 \\
\hline $\mathrm{Ta}$ & 0.555 & 0.013 & 0.523 & 0.03 & -5.71 & 0.543 & 0.0311 & -2.14 & 0.497 & 0.0287 & -10.53 & 0.523 & 0.0351 & -5.71 \\
\hline $\mathrm{Pb}$ & 1.38 & 0.07 & 1.42 & 0.215 & 3.26 & 1.35 & 0.193 & -1.82 & 1.44 & 0.241 & 4.53 & 1.4 & 0.22 & 1.67 \\
\hline Th & 0.548 & 0.011 & 0.526 & 0.0366 & -4.01 & 0.536 & 0.0386 & -2.17 & 0.556 & 0.0409 & 1.39 & 0.538 & 0.0403 & -1.90 \\
\hline $\mathrm{U}$ & 0.442 & 0.018 & 0.455 & 0.0419 & 2.97 & 0.454 & 0.0349 & 2.63 & 0.456 & 0.0401 & 3.22 & 0.455 & 0.0393 & 2.90 \\
\hline
\end{tabular}


Chapter 2.1 LA-ICP-MS Transient Signal Quantification of NIST, MPI-DING, USGS and CGSG Glass Reference Materials by Ratioing, Standardization, and Normalization (RSN)

\section{Table 3g}

Element concentrations of GOR132-G obtained from RSN strategy with NIST 610, StHs6/80-G and GSD-1G as external reference materials. Values are in units of $\mu \mathrm{g} / \mathrm{g}$ except for major elements which are specified in weight percent $(\% \mathrm{~m} / \mathrm{m})$. Standard deviation $(1 \mathrm{~s})$ stems from 78 measurements.

\begin{tabular}{|c|c|c|c|c|c|c|c|c|c|c|c|c|c|c|}
\hline \multirow[t]{2}{*}{ GOR132-G } & \multicolumn{2}{|c|}{ Reference value } & \multicolumn{3}{|c|}{ NIST610 } & \multicolumn{3}{|c|}{ StHs6/80-G } & \multicolumn{3}{|c|}{ GSD-1G } & \multicolumn{3}{|c|}{ Prefer value } \\
\hline & Value & Uncertainty & Average & $1 \mathrm{~s}$ & $\operatorname{RSD}(\%)$ & Average & $1 \mathrm{~s}$ & $\operatorname{RSD}(\%)$ & Average & $1 \mathrm{~s}$ & $\operatorname{RSD}(\%)$ & Average & $1 \mathrm{~s}$ & $\operatorname{RSD}(\%)$ \\
\hline $\mathrm{SiO} 2$ & 45.5 & 0.4 & 48.1 & 0.382 & 5.76 & 45.5 & 0.332 & -0.03 & 45.5 & 0.56 & 0.03 & 46.5 & 1.31 & 2.12 \\
\hline $\mathrm{TiO} 2$ & 0.306 & 0.013 & 0.28 & 0.0045 & -8.59 & 0.298 & 0.0052 & -2.49 & 0.287 & 0.0044 & -6.27 & 0.293 & 0.0076 & -4.15 \\
\hline $\mathrm{Al} 2 \mathrm{O} 3$ & 11 & 0.2 & 10.9 & 0.133 & -0.92 & 10.9 & 0.11 & -0.84 & 10.6 & 0.0969 & -3.47 & 10.8 & 0.171 & -1.59 \\
\hline $\mathrm{FeO}(\mathrm{t})$ & 10.1 & 0.1 & 10.2 & 0.235 & 0.75 & 10.3 & 0.161 & 1.90 & 10.8 & 0.237 & 6.92 & 10.5 & 0.287 & 3.91 \\
\hline $\mathrm{MnO}$ & 0.154 & 0.007 & 0.154 & 0.0032 & 0.01 & 0.157 & 0.0028 & 2.08 & 0.157 & 0.003 & 2.24 & 0.157 & 0.0027 & 2.03 \\
\hline $\mathrm{MgO}$ & 22.4 & 0.2 & 19.8 & 0.238 & -11.47 & 22.5 & 0.211 & 0.46 & 22.3 & 0.227 & -0.62 & 22.4 & 0.251 & -0.05 \\
\hline $\mathrm{CaO}$ & 8.45 & 0.12 & 8.38 & 0.162 & -0.79 & 8.1 & 0.199 & -4.10 & 8.22 & 0.277 & -2.71 & 8.24 & 0.246 & -2.53 \\
\hline $\mathrm{Na} 2 \mathrm{O}$ & 0.83 & 0.04 & 0.874 & 0.028 & 5.25 & 0.802 & 0.0145 & -3.42 & 0.827 & 0.0196 & -0.34 & 0.835 & 0.0378 & 0.56 \\
\hline $\mathrm{K} 2 \mathrm{O}$ & 0.0308 & 0.0034 & 0.0349 & 0.0013 & 13.16 & 0.0293 & 0.001 & -4.88 & 0.0291 & 0.0007 & -5.57 & 0.0292 & 0.0009 & -5.25 \\
\hline $\mathrm{P} 2 \mathrm{O} 5$ & $0.036^{*}$ & $0.012^{*}$ & 0.03 & 0.0046 & -16.68 & 0.0314 & 0.0041 & -12.82 & 0.0274 & 0.0031 & -23.82 & 0.0297 & 0.0042 & -17.53 \\
\hline $\mathrm{Li}$ & 8.9 & 1.2 & 10.2 & 1.17 & 14.89 & 9.69 & 1.12 & 8.91 & 9.74 & 1.23 & 9.39 & 9.88 & 1.18 & 10.97 \\
\hline $\mathrm{Be}$ & $0.08^{*}$ & - & - & - & - & - & - & - & - & - & - & - & - & - \\
\hline B & 17.2 & 2.6 & 20 & 3.6 & 16.44 & 20 & 9.08 & 16.37 & 15 & 2.68 & -12.74 & 18.6 & 6.44 & 8.39 \\
\hline $\mathrm{Sc}$ & 36.5 & 1.2 & 36.4 & 0.8 & -0.26 & 38.5 & 2.23 & 5.45 & 38.1 & 0.686 & 4.48 & 37.6 & 1.74 & 3.09 \\
\hline $\mathrm{V}$ & 214 & 17 & 227 & 3.51 & 6.12 & 234 & 4.55 & 9.44 & 230 & 5.09 & 7.35 & 230 & 5.31 & 7.70 \\
\hline $\mathrm{Cr}$ & 2528 & 183 & 2470 & 38.7 & -2.28 & 3435 & 223 & 35.88 & 2515 & 155 & -0.52 & 2476 & 67.4 & -2.08 \\
\hline Co & 92.7 & 5.7 & 103 & 1.68 & 10.99 & 99.4 & 2.37 & 7.18 & 101 & 1.98 & 9.49 & 101 & 2.52 & 9.15 \\
\hline $\mathrm{Ni}$ & 1187 & 58 & 1353 & 21.4 & 14.03 & 1543 & 84.7 & 29.95 & 1318 & 22.8 & 11.05 & 1338 & 28.5 & 12.69 \\
\hline $\mathrm{Cu}$ & 205 & 21 & 238 & 5.01 & 16.21 & 239 & 7.05 & 16.35 & 230 & 8.59 & 11.99 & 236 & 7.72 & 14.88 \\
\hline $\mathrm{Zn}$ & 76.8 & 12.5 & 79.8 & 4.08 & 3.92 & 76 & 4.15 & -1.06 & 75.2 & 3.02 & -2.10 & 77.2 & 4.34 & 0.48 \\
\hline $\mathrm{Ga}$ & 10.4 & 0.9 & 12 & 0.672 & 15.07 & 11.7 & 0.758 & 12.94 & 11.2 & 0.718 & 7.61 & 11.7 & 0.759 & 12.20 \\
\hline $\mathrm{Rb}$ & 2.1 & 0.1 & 2.33 & 0.175 & 10.84 & 2.25 & 0.189 & 6.92 & 2.1 & 0.156 & 0.16 & 2.23 & 0.198 & 6.37 \\
\hline $\mathrm{Sr}$ & 15.3 & 0.6 & 15.2 & 0.358 & -0.72 & 14.8 & 0.426 & -3.08 & 15.3 & 0.356 & -0.31 & 15.1 & 0.424 & -1.50 \\
\hline $\mathrm{Y}$ & 12.9 & 0.5 & 13 & 0.454 & 0.44 & 12.9 & 0.444 & 0.24 & 13.7 & 0.34 & 6.02 & 13.1 & 0.53 & 1.89 \\
\hline $\mathrm{Zr}$ & 9.9 & 0.3 & 9.81 & 0.379 & -0.94 & 9.82 & 0.436 & -0.80 & 10.2 & 0.371 & 3.52 & 9.93 & 0.437 & 0.28 \\
\hline $\mathrm{Nb}$ & 0.073 & 0.013 & 0.0678 & 0.0307 & -7.06 & 0.0708 & 0.0327 & -2.95 & 0.066 & 0.0315 & -9.59 & 0.0684 & 0.0316 & -6.30 \\
\hline Mo & 30.5 & 2.6 & 33.2 & 5.7 & 8.87 & 41.9 & 11.1 & 37.47 & 32.5 & 5.51 & 6.47 & 33 & 5.68 & 8.10 \\
\hline $\mathrm{Sn}$ & $0.34^{*}$ & $0.09^{*}$ & 0.339 & 0.123 & -0.18 & 0.341 & 0.143 & 0.17 & - & - & - & 0.34 & 0.133 & -0.01 \\
\hline $\mathrm{Sb}$ & 0.06 & 0.04 & - & - & - & - & - & - & - & - & - & - & - & - \\
\hline Cs & 7.45 & 0.63 & 8.29 & 0.234 & 11.25 & 8.53 & 0.376 & 14.53 & 7.95 & 0.238 & 6.72 & 8.29 & 0.372 & 11.21 \\
\hline $\mathrm{Ba}$ & 0.815 & 0.062 & 0.806 & 0.274 & -1.15 & 0.782 & 0.27 & -4.09 & 0.771 & 0.288 & -5.39 & 0.786 & 0.279 & -3.59 \\
\hline $\mathrm{La}$ & 0.0842 & 0.0029 & 0.0913 & 0.022 & 8.38 & 0.0901 & 0.0221 & 7.04 & 0.0934 & 0.0245 & 10.90 & 0.0913 & 0.0229 & 8.38 \\
\hline $\mathrm{Ce}$ & 0.393 & 0.018 & 0.364 & 0.0369 & -7.47 & 0.363 & 0.0379 & -7.56 & 0.371 & 0.0403 & -5.54 & 0.364 & 0.0367 & -7.30 \\
\hline $\operatorname{Pr}$ & 0.089 & 0.004 & 0.0818 & 0.0141 & -8.05 & 0.082 & 0.0142 & -7.82 & 0.0831 & 0.0142 & -6.62 & 0.0822 & 0.0142 & -7.65 \\
\hline $\mathrm{Nd}$ & 0.689 & 0.017 & 0.675 & 0.125 & -2.06 & 0.684 & 0.128 & -0.72 & 0.712 & 0.127 & 3.37 & 0.687 & 0.126 & -0.24 \\
\hline $\mathrm{Sm}$ & 0.508 & 0.015 & 0.511 & 0.0943 & 0.51 & 0.518 & 0.106 & 1.94 & 0.534 & 0.107 & 5.21 & 0.521 & 0.103 & 2.62 \\
\hline $\mathrm{Eu}$ & 0.255 & 0.007 & 0.253 & 0.0428 & -0.78 & 0.252 & 0.0429 & -1.32 & 0.264 & 0.0472 & 3.52 & 0.255 & 0.0442 & 0.03 \\
\hline $\mathrm{Gd}$ & 1.19 & 0.04 & 1.21 & 0.139 & 1.52 & 1.21 & 0.149 & 1.49 & 1.31 & 0.141 & 9.86 & 1.23 & 0.15 & 3.72 \\
\hline $\mathrm{Tb}$ & 0.269 & 0.011 & 0.266 & 0.0224 & -1.03 & 0.27 & 0.0264 & 0.22 & 0.274 & 0.024 & 1.91 & 0.27 & 0.0243 & 0.32 \\
\hline Dy & 2.15 & 0.06 & 2.12 & 0.152 & -1.51 & 2.15 & 0.178 & 0.07 & 2.18 & 0.143 & 1.29 & 2.15 & 0.16 & -0.14 \\
\hline Но & 0.507 & 0.019 & 0.504 & 0.0408 & -0.67 & 0.493 & 0.0435 & -2.69 & 0.518 & 0.0404 & 2.19 & 0.504 & 0.0421 & -0.68 \\
\hline $\mathrm{Er}$ & 1.56 & 0.05 & 1.59 & 0.101 & 1.76 & 1.54 & 0.118 & -1.10 & 1.68 & 0.111 & 7.65 & 1.6 & 0.124 & 2.38 \\
\hline $\mathrm{Tm}$ & 0.234 & 0.009 & 0.227 & 0.0218 & -2.93 & 0.235 & 0.0247 & 0.40 & 0.233 & 0.0235 & -0.53 & 0.231 & 0.0236 & -1.11 \\
\hline $\mathrm{Yb}$ & 1.61 & 0.04 & 1.55 & 0.126 & -3.46 & 1.61 & 0.168 & -0.22 & 1.62 & 0.132 & 0.33 & 1.59 & 0.146 & -1.13 \\
\hline $\mathrm{Lu}$ & 0.237 & 0.009 & 0.233 & 0.0233 & -1.53 & 0.24 & 0.0302 & 1.40 & 0.242 & 0.0249 & 2.18 & 0.238 & 0.0267 & 0.57 \\
\hline $\mathrm{Hf}$ & 0.357 & 0.018 & 0.363 & 0.0702 & 1.58 & 0.362 & 0.0712 & 1.40 & 0.366 & 0.0752 & 2.55 & 0.364 & 0.072 & 1.85 \\
\hline $\mathrm{Ta}$ & 0.031 & 0.002 & - & - & - & - & - & - & - & - & - & - & - & - \\
\hline $\mathrm{Pb}$ & 19.5 & 1.7 & 21.8 & 0.436 & 11.55 & 20.2 & 0.618 & 3.61 & 21.5 & 0.851 & 10.10 & 21.1 & 0.894 & 8.09 \\
\hline Th & 0.009 & 0.003 & 0.0056 & 0.0065 & -38.29 & 0.0055 & 0.0064 & -38.83 & 0.0058 & 0.0069 & -35.66 & 0.0054 & 0.0064 & -39.96 \\
\hline $\mathrm{U}$ & 0.048 & 0.005 & 0.0531 & 0.022 & 10.56 & 0.0521 & 0.0225 & 8.57 & 0.0517 & 0.022 & 7.65 & 0.0526 & 0.0222 & 9.65 \\
\hline
\end{tabular}


Chapter 2.1 LA-ICP-MS Transient Signal Quantification of NIST, MPI-DING, USGS and CGSG Glass Reference Materials by Ratioing, Standardization, and Normalization (RSN)

\section{Table 3h}

Element concentrations of GOR128-G-G obtained from RSN strategy with NIST 610, StHs6/80-G and GSD-1G as external reference materials. Values

are in units of $\mu \mathrm{g} / \mathrm{g}$ except for major elements which are specified in weight percent $(\% \mathrm{~m} / \mathrm{m})$. Standard deviation (1 s) stems from 78 measurements.

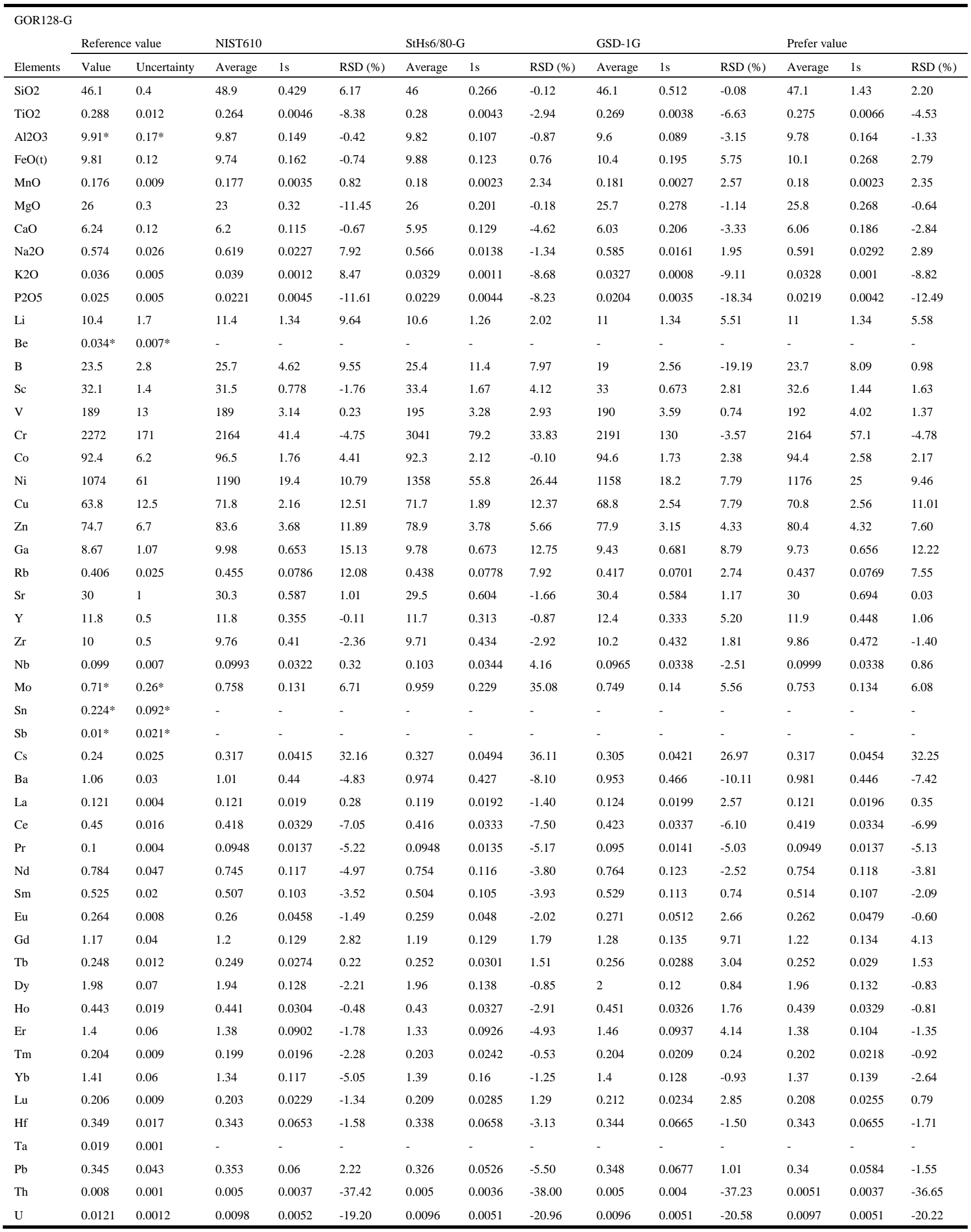


Chapter 2.1 LA-ICP-MS Transient Signal Quantification of NIST, MPI-DING, USGS and CGSG Glass Reference Materials by Ratioing, Standardization, and Normalization (RSN)

\section{Table $3 \mathrm{i}$}

Element concentrations of T1-G obtained from RSN strategy with NIST 610, StHs6/80-G and GSD-1G as external reference materials. Values are in units of $\mu \mathrm{g} / \mathrm{g}$ except for major elements which are specified in weight percent $(\% \mathrm{~m} / \mathrm{m})$. Standard deviation $(1 \mathrm{~s})$ stems from $146 \mathrm{measurements}$.

\begin{tabular}{|c|c|c|c|c|c|c|c|c|c|c|c|c|c|c|}
\hline \multirow[b]{2}{*}{ Elements } & \multicolumn{2}{|c|}{ Reference value } & \multicolumn{3}{|c|}{ NIST610 } & \multicolumn{3}{|c|}{ StHs6/80-G } & \multicolumn{3}{|c|}{ GSD-1G } & \multicolumn{3}{|c|}{ Prefer value } \\
\hline & Value & Uncertainty & Average & $1 \mathrm{~s}$ & $\operatorname{RSD}(\%)$ & Average & $1 \mathrm{~s}$ & $\operatorname{RSD}(\%)$ & Average & $1 \mathrm{~s}$ & $\operatorname{RSD}(\%)$ & Average & $1 \mathrm{~s}$ & $\operatorname{RSD}(\%)$ \\
\hline $\mathrm{SiO} 2$ & 58.6 & 0.4 & 58.8 & 0.48 & 0.30 & 57.5 & 0.411 & -1.85 & 57.5 & 0.322 & -1.90 & 58 & 0.724 & -1.06 \\
\hline $\mathrm{TiO} 2$ & 0.755 & 0.017 & 0.699 & 0.013 & -7.39 & 0.768 & 0.0117 & 1.77 & 0.741 & 0.0096 & -1.81 & 0.756 & 0.0175 & 0.17 \\
\hline $\mathrm{Al} 2 \mathrm{O} 3$ & 17.1 & 0.2 & 17.1 & 0.263 & -0.23 & 17.7 & 0.179 & 3.48 & 17.3 & 0.165 & 1.14 & 17.4 & 0.344 & 1.49 \\
\hline $\mathrm{FeO}(\mathrm{t})$ & 6.44 & 0.06 & 6.42 & 0.178 & -0.36 & 6.8 & 0.0913 & 5.55 & 7.15 & 0.16 & 10.97 & 6.94 & 0.19 & 7.72 \\
\hline $\mathrm{MnO}$ & 0.127 & 0.006 & 0.137 & 0.0029 & 7.65 & 0.144 & 0.0019 & 13.56 & 0.145 & 0.0031 & 14.05 & 0.144 & 0.002 & 13.57 \\
\hline $\mathrm{MgO}$ & 3.75 & 0.04 & 3.37 & 0.0695 & -10.24 & 3.94 & 0.0591 & 5.12 & 3.9 & 0.0609 & 4.07 & 3.92 & 0.0631 & 4.60 \\
\hline $\mathrm{CaO}$ & 7.1 & 0.09 & 7.42 & 0.389 & 4.55 & 7.4 & 0.396 & 4.20 & 7.49 & 0.198 & 5.46 & 7.43 & 0.349 & 4.60 \\
\hline $\mathrm{Na} 2 \mathrm{O}$ & 3.13 & 0.09 & 3.14 & 0.075 & 0.23 & 2.98 & 0.0323 & -4.81 & 3.07 & 0.0481 & -1.79 & 3.06 & 0.0873 & -2.15 \\
\hline $\mathrm{K} 2 \mathrm{O}$ & 1.96 & 0.04 & 2.2 & 0.0913 & 12.08 & 1.93 & 0.0387 & -1.73 & 1.9 & 0.0375 & -3.20 & 1.91 & 0.0405 & -2.32 \\
\hline $\mathrm{P} 2 \mathrm{O} 5$ & 0.168 & 0.026 & 0.193 & 0.0109 & 15.00 & 0.208 & 0.0079 & 24.02 & 0.181 & 0.0053 & 7.91 & 0.197 & 0.0148 & 17.14 \\
\hline $\mathrm{Li}$ & 19.9 & 0.9 & 22.4 & 1.57 & 12.51 & 21.8 & 1.87 & 9.65 & 22.7 & 1.82 & 13.93 & 22.3 & 1.78 & 11.95 \\
\hline $\mathrm{Be}$ & $2^{*}$ & $0.6^{*}$ & 3.18 & 2.87 & 58.97 & - & - & - & 3.35 & 3.29 & 67.70 & 3.29 & 3.1 & 64.55 \\
\hline B & $4.1^{*}$ & $1.1^{*}$ & 5.7 & 4.48 & 38.97 & 6.17 & 6.89 & 50.38 & - & - & - & 5.51 & 5.29 & 34.45 \\
\hline $\mathrm{Sc}$ & 26.9 & 1.1 & 25.6 & 0.799 & -4.96 & 28.3 & 1.1 & 5.25 & 27.7 & 0.806 & 2.99 & 27.1 & 1.5 & 0.82 \\
\hline V & 190 & 11 & 198 & 3.22 & 4.16 & 211 & 3.34 & 11.24 & 206 & 2.64 & 8.61 & 205 & 6.55 & 7.93 \\
\hline $\mathrm{Cr}$ & 20.9 & 2 & 20.8 & 1.36 & -0.46 & 30.8 & 2.31 & 47.42 & 21.8 & 1.9 & 4.46 & 21.1 & 1.55 & 1.17 \\
\hline Co & 18.9 & 0.8 & 20.6 & 0.533 & 9.19 & 20.6 & 0.549 & 8.85 & 21.1 & 0.531 & 11.40 & 20.7 & 0.551 & 9.58 \\
\hline $\mathrm{Ni}$ & 10.6 & 1.3 & 10.8 & 1.83 & 1.64 & 12.8 & 2.12 & 20.37 & 10.8 & 1.99 & 1.67 & 10.7 & 1.72 & 0.53 \\
\hline $\mathrm{Cu}$ & 18.8 & 2 & 20.5 & 1.01 & 9.29 & 21.4 & 1.05 & 13.93 & 20.5 & 1.22 & 9.06 & 20.8 & 1.16 & 10.71 \\
\hline $\mathrm{Zn}$ & 74 & 10 & 86.9 & 3.7 & 17.44 & 85.3 & 3.48 & 15.27 & 83.7 & 2.78 & 13.11 & 85.4 & 3.62 & 15.44 \\
\hline $\mathrm{Ga}$ & 19.4 & 0.9 & 21.8 & 1.26 & 12.51 & 22.3 & 1.09 & 14.86 & 21.4 & 1.25 & 10.42 & 21.9 & 1.25 & 12.70 \\
\hline $\mathrm{Rb}$ & 79.7 & 3.5 & 84.8 & 2.56 & 6.46 & 84.8 & 1.65 & 6.35 & 79.7 & 1.22 & 0.00 & 83.4 & 3.01 & 4.62 \\
\hline $\mathrm{Sr}$ & 284 & 6 & 290 & 5.67 & 1.98 & 293 & 4.13 & 3.06 & 302 & 4.45 & 6.32 & 294 & 6.62 & 3.51 \\
\hline $\mathrm{Y}$ & 23.9 & 0.8 & 24.2 & 0.803 & 1.38 & 25 & 0.564 & 4.68 & 26.5 & 0.755 & 10.82 & 25.1 & 1.12 & 5.12 \\
\hline $\mathrm{Zr}$ & 144 & 4 & 138 & 4.07 & -4.21 & 142 & 2.92 & -1.25 & 150 & 3.11 & 3.91 & 143 & 5.65 & -0.94 \\
\hline $\mathrm{Nb}$ & 8.87 & 0.43 & 8.85 & 0.24 & -0.26 & 9.52 & 0.291 & 7.34 & 8.84 & 0.251 & -0.34 & 9.08 & 0.421 & 2.41 \\
\hline Mo & 4.2 & 1.8 & 1.4 & 0.15 & -66.71 & 1.85 & 0.386 & -56.05 & 1.42 & 0.156 & -66.14 & 1.4 & 0.149 & -66.63 \\
\hline Sn & 2 & 0.5 & 2.38 & 0.19 & 19.11 & 2.4 & 0.302 & 19.86 & 1.93 & 0.163 & -3.58 & 2.39 & 0.252 & 19.48 \\
\hline $\mathrm{Sb}$ & 0.25 & 0.05 & 0.298 & 0.111 & 19.28 & 0.294 & 0.158 & 17.53 & 0.336 & 0.129 & 34.58 & 0.298 & 0.111 & 19.28 \\
\hline Cs & 2.69 & 0.19 & 2.86 & 0.143 & 6.32 & 3.07 & 0.217 & 14.18 & 2.84 & 0.12 & 5.70 & 2.93 & 0.197 & 9.04 \\
\hline $\mathrm{Ba}$ & 388 & 12 & 402 & 8.25 & 3.67 & 402 & 6.94 & 3.63 & 406 & 8.35 & 4.62 & 403 & 7.56 & 3.83 \\
\hline $\mathrm{La}$ & 70.4 & 2.4 & 73.5 & 1.73 & 4.45 & 74.9 & 1.46 & 6.33 & 77.6 & 1.73 & 10.25 & 75.1 & 2.21 & 6.65 \\
\hline $\mathrm{Ce}$ & 127 & 4 & 131 & 2.27 & 3.48 & 136 & 3.36 & 7.38 & 138 & 3 & 8.71 & 135 & 3.9 & 6.26 \\
\hline $\operatorname{Pr}$ & 12.4 & 0.4 & 12.9 & 0.303 & 3.87 & 13.4 & 0.293 & 7.79 & 13.4 & 0.3 & 8.39 & 13.2 & 0.383 & 6.48 \\
\hline $\mathrm{Nd}$ & 41.4 & 1.2 & 42.4 & 1.36 & 2.45 & 44.8 & 1.32 & 8.24 & 45.8 & 1.38 & 10.70 & 44.2 & 1.93 & 6.75 \\
\hline $\mathrm{Sm}$ & 6.57 & 0.14 & 6.81 & 0.327 & 3.61 & 7.11 & 0.492 & 8.20 & 7.35 & 0.352 & 11.83 & 7.06 & 0.456 & 7.53 \\
\hline $\mathrm{Eu}$ & 1.21 & 0.04 & 1.25 & 0.0756 & 3.02 & 1.3 & 0.0964 & 7.14 & 1.34 & 0.0796 & 10.48 & 1.29 & 0.09 & 6.41 \\
\hline $\mathrm{Gd}$ & 5.31 & 0.29 & 5.33 & 0.359 & 0.29 & 5.45 & 0.428 & 2.71 & 5.89 & 0.379 & 10.86 & 5.52 & 0.447 & 4.01 \\
\hline $\mathrm{Tb}$ & 0.773 & 0.029 & 0.744 & 0.0435 & -3.76 & 0.788 & 0.0615 & 1.95 & 0.794 & 0.047 & 2.65 & 0.773 & 0.0562 & 0.05 \\
\hline Dy & 4.5 & 0.12 & 4.48 & 0.196 & -0.51 & 4.71 & 0.321 & 4.68 & 4.77 & 0.195 & 5.98 & 4.64 & 0.276 & 3.11 \\
\hline Ho & 0.86 & 0.031 & 0.89 & 0.0506 & 3.53 & 0.905 & 0.0561 & 5.29 & 0.946 & 0.0528 & 10.05 & 0.911 & 0.0575 & 5.89 \\
\hline $\mathrm{Er}$ & 2.49 & 0.08 & 2.55 & 0.155 & 2.21 & 2.58 & 0.184 & 3.45 & 2.79 & 0.17 & 11.98 & 2.62 & 0.2 & 5.40 \\
\hline $\mathrm{Tm}$ & 0.354 & 0.015 & 0.361 & 0.0325 & 2.06 & 0.387 & 0.0378 & 9.33 & 0.381 & 0.032 & 7.75 & 0.376 & 0.0357 & 6.12 \\
\hline $\mathrm{Yb}$ & 2.38 & 0.08 & 2.38 & 0.161 & -0.09 & 2.59 & 0.255 & 8.83 & 2.57 & 0.157 & 7.94 & 2.51 & 0.221 & 5.36 \\
\hline $\mathrm{Lu}$ & 0.354 & 0.012 & 0.352 & 0.0295 & -0.51 & 0.373 & 0.041 & 5.48 & 0.377 & 0.0307 & 6.42 & 0.366 & 0.0359 & 3.48 \\
\hline $\mathrm{Hf}$ & 3.88 & 0.15 & 3.69 & 0.244 & -5.01 & 3.76 & 0.267 & -3.07 & 3.83 & 0.273 & -1.36 & 3.75 & 0.266 & -3.30 \\
\hline $\mathrm{Ta}$ & 0.464 & 0.021 & 0.447 & 0.0268 & -3.73 & 0.465 & 0.0333 & 0.31 & 0.433 & 0.0278 & -6.58 & 0.45 & 0.0323 & -3.01 \\
\hline $\mathrm{Pb}$ & 11.6 & 1.5 & 16.2 & 0.436 & 40.07 & 15.7 & 0.453 & 35.55 & 16.5 & 0.72 & 41.85 & 16.1 & 0.536 & 38.61 \\
\hline Th & 31.3 & 1 & 32.3 & 1.01 & 3.23 & 33.3 & 0.83 & 6.38 & 34.9 & 1.06 & 11.50 & 33.4 & 1.35 & 6.55 \\
\hline $\mathrm{U}$ & 1.71 & 0.1 & 1.97 & 0.0761 & 15.43 & 2.01 & 0.0812 & 17.68 & 2.01 & 0.0918 & 17.51 & 2 & 0.0845 & 16.74 \\
\hline
\end{tabular}


Chapter 2.1 LA-ICP-MS Transient Signal Quantification of NIST, MPI-DING, USGS and CGSG Glass Reference Materials by Ratioing, Standardization, and Normalization (RSN)

\section{Table $3 \mathrm{j}$}

Element concentrations of StHs6/80-G obtained from RSN strategy with NIST 610, StHs6/80-G and GSD-1G as external reference materials. Values are in units of $\mu \mathrm{g} / \mathrm{g}$ except for major elements which are specified in weight percent $(\% \mathrm{~m} / \mathrm{m})$. Standard deviation $(1 \mathrm{~s})$ stems from 78 measurements.

\begin{tabular}{|c|c|c|c|c|c|c|c|c|c|c|c|c|c|c|}
\hline \multicolumn{15}{|l|}{ StHs6/80-G } \\
\hline \multirow[b]{2}{*}{ Elements } & \multicolumn{2}{|c|}{ Reference value } & \multicolumn{3}{|l|}{ NIST610 } & \multicolumn{3}{|c|}{ StHs6/80-G } & \multicolumn{3}{|l|}{ GSD-1G } & \multicolumn{3}{|c|}{ Prefer value } \\
\hline & Value & Uncertainty & Average & $1 \mathrm{~s}$ & $\operatorname{RSD}(\%)$ & Average & $1 \mathrm{~s}$ & $\operatorname{RSD}(\%)$ & Average & $1 \mathrm{~s}$ & $\operatorname{RSD}(\%)$ & Average & $1 \mathrm{~s}$ & $\operatorname{RSD}(\%)$ \\
\hline $\mathrm{TiO} 2$ & 0.703 & 0.021 & 0.634 & 0.0163 & -9.88 & 0.701 & 0.0054 & -0.22 & 0.674 & 0.012 & -4.17 & 0.69 & 0.0164 & -1.92 \\
\hline $\mathrm{Al} 2 \mathrm{O} 3$ & 17.8 & 0.2 & 17 & 0.22 & -4.77 & 17.7 & 0.0712 & -0.29 & 17.3 & 0.197 & -2.79 & 17.3 & 0.382 & -2.60 \\
\hline $\mathrm{FeO}(\mathrm{t})$ & 4.37 & 0.07 & 4.11 & 0.126 & -6.01 & 4.36 & 0.0288 & -0.30 & 4.59 & 0.0848 & 4.96 & 4.46 & 0.128 & 1.96 \\
\hline $\mathrm{MnO}$ & 0.076 & 0.004 & 0.0717 & 0.0019 & -5.72 & 0.0758 & 0.0005 & -0.21 & 0.0762 & 0.0013 & 0.22 & 0.076 & 0.0009 & -0.03 \\
\hline $\mathrm{MgO}$ & 1.97 & 0.04 & 1.66 & 0.0309 & -15.66 & 1.96 & 0.0123 & -0.31 & 1.95 & 0.034 & -1.12 & 1.96 & 0.0254 & -0.66 \\
\hline $\mathrm{CaO}$ & 5.28 & 0.09 & 5.27 & 0.196 & -0.27 & 5.28 & 0.106 & -0.04 & 5.3 & 0.254 & 0.31 & 5.28 & 0.189 & -0.03 \\
\hline $\mathrm{Na} 2 \mathrm{O}$ & 4.44 & 0.14 & 4.64 & 0.0868 & 4.44 & 4.43 & 0.0304 & -0.21 & 4.57 & 0.0437 & 2.92 & 4.54 & 0.108 & 2.33 \\
\hline $\mathrm{K} 2 \mathrm{O}$ & 1.29 & 0.02 & 1.47 & 0.0833 & 14.33 & 1.29 & 0.0096 & -0.18 & 1.26 & 0.0499 & -2.55 & 1.27 & 0.0368 & -1.20 \\
\hline $\mathrm{P} 2 \mathrm{O} 5$ & 0.164 & 0.018 & 0.151 & 0.0093 & -7.66 & 0.164 & 0.0032 & -0.15 & 0.142 & 0.0052 & -13.62 & 0.154 & 0.0117 & -5.94 \\
\hline $\mathrm{Li}$ & 20.7 & 2.3 & 20.9 & 1.76 & 1.14 & 20.7 & 1.15 & 0.09 & 20.9 & 1.96 & 0.95 & 20.8 & 1.64 & 0.71 \\
\hline B & 11.8 & 1.3 & 12.8 & 4.08 & 8.83 & 12.1 & 4.91 & 2.41 & 10 & 2.78 & -15.26 & 11.8 & 4.27 & -0.09 \\
\hline $\mathrm{Sc}$ & 11.5 & 0.8 & 10.4 & 0.695 & -9.22 & 11.5 & 0.274 & -0.11 & 11.3 & 0.687 & -1.43 & 11.1 & 0.748 & -3.78 \\
\hline $\mathrm{V}$ & 90.3 & 6.7 & 84.1 & 2.36 & -6.90 & 90.1 & 0.78 & -0.23 & 88.2 & 1.84 & -2.32 & 87.4 & 3.16 & -3.22 \\
\hline $\mathrm{Cr}$ & 16.9 & 3.3 & 11.7 & 1.06 & -30.90 & 16.9 & 1.1 & 0.17 & 12.2 & 1.25 & -28.09 & 11.9 & 1.17 & -29.69 \\
\hline Co & 13.2 & 1.1 & 13.1 & 0.516 & -0.65 & 13.2 & 0.286 & -0.11 & 13.4 & 0.451 & 1.74 & 13.2 & 0.445 & 0.20 \\
\hline $\mathrm{Ni}$ & 23.7 & 3.8 & 20.3 & 1.95 & -14.53 & 23.7 & 1.58 & 0.12 & 20.2 & 1.73 & -14.70 & 20.2 & 1.86 & -14.60 \\
\hline $\mathrm{Cu}$ & 41.5 & 8.3 & 39.8 & 1.69 & -3.99 & 41.4 & 0.983 & -0.17 & 40 & 1.74 & -3.72 & 40.4 & 1.66 & -2.53 \\
\hline $\mathrm{Zn}$ & 67 & 7 & 68.6 & 4.88 & 2.35 & 67 & 2.51 & 0.07 & 65.5 & 3.85 & -2.27 & 67.2 & 4.06 & 0.26 \\
\hline $\mathrm{Ga}$ & 20.9 & 2.7 & 20.4 & 1.16 & -2.37 & 20.9 & 0.591 & -0.04 & 19.9 & 1.02 & -4.90 & 20.4 & 1.03 & -2.21 \\
\hline $\mathrm{Rb}$ & 30.7 & 1.7 & 30.8 & 1.07 & 0.26 & 30.7 & 0.394 & -0.16 & 28.9 & 0.736 & -5.92 & 30.2 & 1.14 & -1.58 \\
\hline $\mathrm{Sr}$ & 482 & 8 & 474 & 13.1 & -1.71 & 481 & 3.82 & -0.25 & 495 & 8.22 & 2.71 & 482 & 12.6 & 0.03 \\
\hline $\mathrm{Y}$ & 11.4 & 0.4 & 11 & 0.416 & -3.58 & 11.4 & 0.215 & -0.27 & 12 & 0.416 & 5.42 & 11.4 & 0.54 & 0.08 \\
\hline $\mathrm{Zr}$ & 118 & 3 & 114 & 3.6 & -3.59 & 118 & 1.22 & -0.26 & 123 & 3.33 & 4.39 & 118 & 4.71 & -0.20 \\
\hline Mo & 2 & 0.6 & 1.57 & 0.281 & -21.73 & 2.01 & 0.205 & 0.72 & 1.64 & 0.282 & -17.91 & 1.6 & 0.284 & -20.09 \\
\hline Sn & 1.1 & 0.2 & 1.11 & 0.175 & 0.75 & 1.11 & 0.101 & 0.50 & 0.892 & 0.148 & -18.89 & 1.11 & 0.143 & 0.63 \\
\hline $\mathrm{Sb}$ & 0.2 & 0.07 & 0.273 & 0.181 & 36.54 & - & - & - & 0.326 & 0.233 & 63.03 & 0.273 & 0.181 & 36.54 \\
\hline $\mathrm{Cs}$ & 1.75 & 0.11 & 1.63 & 0.0862 & -7.13 & 1.75 & 0.0547 & -0.14 & 1.62 & 0.0902 & -7.68 & 1.67 & 0.0986 & -4.74 \\
\hline $\mathrm{Ba}$ & 298 & 9 & 297 & 7.92 & -0.33 & 297 & 2.89 & -0.24 & 299 & 6.91 & 0.38 & 298 & 6.3 & -0.10 \\
\hline $\mathrm{La}$ & 12 & 0.3 & 11.7 & 0.392 & -2.25 & 12 & 0.163 & -0.32 & 12.4 & 0.329 & 3.51 & 12 & 0.413 & 0.02 \\
\hline $\mathrm{Ce}$ & 26.1 & 0.7 & 25.1 & 0.756 & -3.74 & 26 & 0.235 & -0.21 & 26.4 & 0.561 & 1.27 & 25.8 & 0.781 & -1.08 \\
\hline $\operatorname{Pr}$ & 3.2 & 0.06 & 3.06 & 0.11 & -4.30 & 3.2 & 0.0683 & -0.09 & 3.22 & 0.107 & 0.62 & 3.15 & 0.119 & -1.42 \\
\hline $\mathrm{Nd}$ & 13 & 0.3 & 12.4 & 0.61 & -4.40 & 13 & 0.385 & 0.00 & 13.5 & 0.675 & 3.75 & 12.9 & 0.701 & -0.57 \\
\hline $\mathrm{Sm}$ & 2.78 & 0.05 & 2.69 & 0.21 & -3.15 & 2.78 & 0.142 & -0.03 & 2.9 & 0.234 & 4.45 & 2.78 & 0.213 & 0.06 \\
\hline $\mathrm{Eu}$ & 0.953 & 0.022 & 0.921 & 0.0777 & -3.36 & 0.954 & 0.0553 & 0.10 & 0.987 & 0.0887 & 3.58 & 0.951 & 0.0784 & -0.20 \\
\hline $\mathrm{Gd}$ & 2.59 & 0.09 & 2.51 & 0.208 & -2.96 & 2.59 & 0.138 & -0.15 & 2.79 & 0.238 & 7.79 & 2.62 & 0.225 & 1.00 \\
\hline $\mathrm{Tb}$ & 0.371 & 0.011 & 0.358 & 0.031 & -3.53 & 0.373 & 0.0218 & 0.41 & 0.382 & 0.0335 & 2.89 & 0.37 & 0.0304 & -0.34 \\
\hline Dy & 2.22 & 0.06 & 2.12 & 0.154 & -4.51 & 2.22 & 0.111 & -0.18 & 2.27 & 0.159 & 2.35 & 2.2 & 0.155 & -1.06 \\
\hline Но & 0.42 & 0.011 & 0.414 & 0.036 & -1.53 & 0.42 & 0.0246 & 0.09 & 0.44 & 0.0375 & 4.71 & 0.423 & 0.0345 & 0.77 \\
\hline Er & 1.18 & 0.04 & 1.17 & 0.0986 & -0.90 & 1.18 & 0.0656 & 0.05 & 1.29 & 0.109 & 9.53 & 1.21 & 0.105 & 2.30 \\
\hline $\mathrm{Tm}$ & 0.172 & 0.007 & 0.161 & 0.0197 & -6.47 & 0.173 & 0.0162 & 0.69 & 0.17 & 0.0223 & -1.43 & 0.168 & 0.02 & -2.49 \\
\hline $\mathrm{Yb}$ & 1.13 & 0.03 & 1.06 & 0.126 & -6.27 & 1.14 & 0.0992 & 0.50 & 1.15 & 0.146 & 1.96 & 1.11 & 0.13 & -1.56 \\
\hline $\mathrm{Lu}$ & 0.168 & 0.006 & 0.16 & 0.018 & -4.95 & 0.17 & 0.0152 & 1.32 & 0.171 & 0.02 & 2.03 & 0.167 & 0.0184 & -0.76 \\
\hline $\mathrm{Hf}$ & 3.07 & 0.09 & 2.99 & 0.197 & -2.60 & 3.07 & 0.142 & 0.00 & 3.11 & 0.22 & 1.34 & 3.05 & 0.192 & -0.58 \\
\hline $\mathrm{Ta}$ & 0.42 & 0.015 & 0.4 & 0.0318 & -4.71 & 0.422 & 0.0254 & 0.49 & 0.388 & 0.0327 & -7.59 & 0.405 & 0.033 & -3.61 \\
\hline $\mathrm{Pb}$ & 10.3 & 0.9 & 10.8 & 0.828 & 4.72 & 10.3 & 0.643 & 0.43 & 11 & 0.965 & 7.19 & 10.7 & 0.857 & 3.84 \\
\hline Th & 2.28 & 0.07 & 2.2 & 0.0922 & -3.47 & 2.28 & 0.0536 & -0.18 & 2.38 & 0.113 & 4.21 & 2.28 & 0.111 & -0.17 \\
\hline $\mathrm{U}$ & 1.01 & 0.04 & 0.993 & 0.0571 & -1.70 & 1.01 & 0.0391 & 0.05 & 1.01 & 0.0535 & 0.40 & 1.01 & 0.0511 & -0.49 \\
\hline
\end{tabular}


Chapter 2.1 LA-ICP-MS Transient Signal Quantification of NIST, MPI-DING, USGS and CGSG Glass Reference Materials by Ratioing, Standardization, and Normalization (RSN)

\section{Table $3 \mathrm{k}$}

Element concentrations of BIR-1G obtained from RSN strategy with NIST 610, StHs6/80-G and GSD-1G as external reference materials. Values are in

units of $\mu \mathrm{g} / \mathrm{g}$ except for major elements which are specified in weight percent $(\% \mathrm{~m} / \mathrm{m})$. Standard deviation $(1 \mathrm{~s})$ stems from 74 measurements

BIR-1G

\begin{tabular}{|c|c|c|c|c|c|c|c|c|c|c|c|c|c|c|}
\hline \multirow[b]{2}{*}{ Elements } & \multicolumn{2}{|c|}{ Reference value } & \multicolumn{2}{|l|}{ NIST610 } & \multicolumn{4}{|c|}{ StHs6/80-G } & \multicolumn{3}{|l|}{ GSD-1G } & \multicolumn{3}{|c|}{ Prefer value } \\
\hline & Value & Uncertainty & Average & $1 \mathrm{~s}$ & $\operatorname{RSD}(\%)$ & Average & $1 \mathrm{~s}$ & $\operatorname{RSD}(\%)$ & Average & $1 \mathrm{~s}$ & $\operatorname{RSD}(\%)$ & Average & $1 \mathrm{~s}$ & $\operatorname{RSD}(\%)$ \\
\hline $\mathrm{SiO} 2$ & 47.5 & 0.3 & 50 & 0.671 & 5.22 & 48.1 & 0.634 & 1.31 & 48 & 0.681 & 1.09 & 48.8 & 1.12 & 2.64 \\
\hline $\mathrm{TiO} 2$ & 1.04 & 0.07 & 0.887 & 0.0265 & -14.75 & 0.963 & 0.0209 & -7.40 & 0.923 & 0.0161 & -11.28 & 0.945 & 0.0276 & -9.17 \\
\hline $\mathrm{Al} 2 \mathrm{O} 3$ & 15.5 & 0.2 & 15.1 & 0.233 & -2.87 & 15.4 & 0.206 & -0.75 & 15 & 0.213 & -3.52 & 15.1 & 0.285 & -2.31 \\
\hline $\mathrm{FeO}(\mathrm{t})$ & 10.4 & 0.1 & 9.95 & 0.264 & -4.35 & 10.4 & 0.206 & -0.28 & 10.9 & 0.276 & 4.49 & 10.6 & 0.334 & 1.84 \\
\hline $\mathrm{MnO}$ & 0.19 & 0.01 & 0.174 & 0.0057 & -8.27 & 0.182 & 0.0039 & -4.47 & 0.181 & 0.0048 & -4.59 & 0.181 & 0.0043 & -4.56 \\
\hline $\mathrm{MgO}$ & 9.4 & 0.1 & 8.45 & 0.153 & -10.14 & 9.76 & 0.103 & 3.80 & 9.67 & 0.18 & 2.89 & 9.71 & 0.146 & 3.35 \\
\hline $\mathrm{CaO}$ & 13.3 & 0.2 & 12.8 & 0.35 & -3.85 & 12.6 & 0.363 & -5.52 & 12.7 & 0.393 & -4.67 & 12.7 & 0.38 & -4.69 \\
\hline $\mathrm{Na} 2 \mathrm{O}$ & 1.85 & 0.07 & 1.91 & 0.0656 & 3.02 & 1.79 & 0.0478 & -3.49 & 1.84 & 0.0491 & -0.81 & 1.84 & 0.0749 & -0.41 \\
\hline $\mathrm{K} 2 \mathrm{O}$ & 0.03 & 0.005 & 0.0242 & 0.0016 & -19.27 & 0.0209 & 0.0013 & -30.39 & 0.0206 & 0.001 & -31.31 & 0.0207 & 0.0012 & -30.84 \\
\hline $\mathrm{P} 2 \mathrm{O} 5$ & 0.027 & 0.003 & 0.0195 & 0.0054 & -27.93 & 0.0204 & 0.0053 & -24.39 & 0.0177 & 0.0049 & -34.49 & 0.0192 & 0.0053 & -29.02 \\
\hline $\mathrm{Li}$ & 3 & 0.7 & 3.14 & 1.31 & 4.52 & 3.02 & 1.28 & 0.77 & 3.18 & 1.38 & 5.93 & 3.1 & 1.32 & 3.31 \\
\hline
\end{tabular}

$\mathrm{Be} \quad 0.1^{*} \quad-$

B

Sc $\quad 43 \quad 3$

V $\quad 326 \quad 32$

Cr $\quad 392 \quad 24$

Co $\quad 52 \quad 5$

$\mathrm{Ni} \quad 178 \quad 18$

$\begin{array}{lll}\mathrm{Cu} & 119 \quad 12\end{array}$

$\begin{array}{lll}\mathrm{Zn} & 78 \quad 17\end{array}$

Ga $\quad 15 \quad 2$

$\begin{array}{lll}\mathrm{Rb} & 0.197 \quad 0.007\end{array}$

$\begin{array}{llll}\mathrm{Sr} & 109 & 2 & 105\end{array}$

$\begin{array}{lll}\mathrm{Y} & 14.3 & 1.4\end{array}$

$\begin{array}{lll}\mathrm{Zr} & 14 & 1.2\end{array}$

$\begin{array}{lll}\mathrm{Nb} & 0.52 \quad 0.04\end{array}$

Mo $0.075^{*} \quad 0.011^{*}$

Sn $2.3^{*} \quad 1.3^{*}$

$\begin{array}{lll}\mathrm{Sb} & 0.56 \quad 0.09\end{array}$

Cs $\quad 0.007 \quad 0.002$

$\begin{array}{lll}\mathrm{Ba} & 6.5 & 0.07\end{array}$

$\begin{array}{lll}\mathrm{La} & 0.609 & 0.02\end{array}$

$\begin{array}{lll}\mathrm{Ce} & 1.89 & 0.04\end{array}$

$\begin{array}{lll}\text { Pr } & 0.37 \quad 0.02\end{array}$

$\begin{array}{lll}\text { Nd } & 2.37 \quad 0.03\end{array}$

Sm $\quad 1.09 \quad 0.02$

$\begin{array}{lll}\mathrm{Eu} & 0.517 \quad 0.005\end{array}$

Gd $\quad 1.85 \quad 0.02$

$\begin{array}{lll}\mathrm{Tb} & 0.35 & 0.04\end{array}$

Dy $\quad 2.55 \quad 0.02$

Ho $\quad 0.56 \quad 0.03$

$\begin{array}{lll}\text { Er } & 1.7 \quad 0.02\end{array}$

$\begin{array}{lll}\mathrm{Tm} & 0.24 & 0.03\end{array}$

$\begin{array}{lll}\mathrm{Yb} & 1.64 \quad 0.03\end{array}$

$\begin{array}{lll}\mathrm{Lu} & 0.248 & 0.009\end{array}$

$\begin{array}{lll}\text { Hf } & 0.57 \quad 0.03\end{array}$

$\begin{array}{lll}\text { Ta } & 0.036 & 0.006\end{array}$

$41.8-1.52$

$330 \quad 8.29$

$52 \quad-2.74$

$392 \quad 1.25$

$\begin{array}{lll}56 & 1.37 & 7.69\end{array}$

$\begin{array}{lll}191 & 5.69 & 7.07\end{array}$

$130 \quad 4.61 \quad 9.29$

$\begin{array}{lll}90.4 & 5.28 & 15.90\end{array}$

$16.7 \quad 0.666 \quad 11.34$

105

2.99

13.

0.523

$\begin{array}{ll}0.552 & -3.09\end{array}$

$0.619 \quad-6.52$

\section{$-$}

$\begin{array}{lll}46 & 1.86 & 6.86\end{array}$

$-$

$561 \quad 34$

$55.3 \quad 1.47$

$222 \quad 9.94$

$134 \quad 3.84$

$88.4 \quad 3.93$

6.86

$\begin{array}{lll}44.6 & 1.37 \quad 3.79\end{array}$

44.1

43.02

337
402

$5.48 \quad 3.44$

338

$2.39 \quad 2.56$

$\begin{array}{lll}02 & 12.4 & 2.54\end{array}$

396

$13.1 \quad 1.10$

6.41

55.9

$0.936 \quad 7.48$

55.7

189

1.33

12.76

$\begin{array}{lll}188 & 4.4 & 5.36 \\ 127 & 6.12 & 6.88\end{array}$

$5.34-6.25$

13.37

$\begin{array}{lll}85.5 & 2.42 & 9.61\end{array}$

88.3

5.62

$4.56 \quad 13.16$

$\begin{array}{lll}16.9 & 0.886 & 12.87\end{array}$

$\begin{array}{lll}16 & 0.545 \quad 6.83\end{array}$

$\begin{array}{lll}16.6 & 0.809 & 10.57\end{array}$

$\begin{array}{lll}\mathrm{Pb} & 3.7 & 0.3\end{array}$

$\begin{array}{lll}\text { Th } & 0.03 \quad 0.002\end{array}$

0.857

$0.0497 \quad 0.54$

0.576

$0.138 \quad-62.73$

$0.123 \quad 2.92$

6.34

0.58

$0.639-2.40$

$0.0495 \quad-4.76$

$\begin{array}{ll}0.0495 & -4.76 \\ 0.1 & -2.74\end{array}$

0.352

$0.0368 \quad-4.84$

2.26

$0.227 \quad-4.47$

105

1.91

14.2

$\begin{array}{ll}1.91 & -3.44 \\ 0.409 & -0.63\end{array}$

108

\begin{tabular}{ll}
$0.51-4.50$ \\
\hline
\end{tabular}

14.9

2.65

$-$

$-$

$-$

$\begin{array}{lll}\mathrm{U} & 0.023 \quad 0.006\end{array}$

$\begin{array}{lll}0.5 & 0.0585 & -3.35\end{array}$

$\begin{array}{lll}1.79 & 0.221 & -3.47\end{array}$

$\begin{array}{ll}0.221 & -3.47 \\ 0.0225 & -7.73\end{array}$

$0.202-6.05$

2.4

0.037

$-3.83$

$\begin{array}{lll}1.61 & 0.113 & -5.21\end{array}$

$\begin{array}{lll}0.236 & 0.0237 & -1.62\end{array}$

$\begin{array}{lll}1.55 & 0.16 & -5.24 \\ 0.237 & 0.0278 & -4.52\end{array}$

0.556

$0.0555 \quad 6$

$$
14
$$

$0.432 \quad 4.30$

14.3

$2.79-2.66$

0.868

0.868

$0.156-62.25$

$0.512 \quad 0.0511--1.58$

13.5

$0.629-0.07$

$0.663 \quad-3.91$ 0.578

$\begin{array}{lll}6.25 & 0.614 & -3.86\end{array}$

0.688

$\begin{array}{ll}- & - \\ 0.112 & -70.07\end{array}$

$\begin{array}{lll}0.531 & 0.0556 \quad 2.15\end{array}$

-

$0.147 \quad-62.49$

$\begin{array}{llllll}0.644 & 0.148 & 14.95 & 0.576 & 0.123 & 2.92\end{array}$

0.237

$0.0278 \quad-4.52$

0.586

$0.0493-3.79$

6.24

$-$

$\begin{array}{lll}1.88 & 0.0832 & -0.50\end{array}$

0.606

$0.634-4.05$

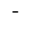

$6.28 \quad 0.632-3.42$

0.359

$0.0363-3.06$

1.9
0.361

$0.0505 \quad-0.55$

0.59

$0.051-3.15$

2.38

$0.238 \quad 0.41$

2.42

$0.0382-2.41$

1.87

$0.0954-0.93$

$\begin{array}{lll}1.12 & 0.197 & 2.49\end{array}$

1.17

$0.236 \quad 2.09$

0.357

$0.0373 \quad-3.51$

$\begin{array}{lll}0.513 & 0.06 & -0.85\end{array}$

0.529

$0.169 \quad 7.31$

$\begin{array}{lll}2.35 & 0.243 \quad-0.76\end{array}$

$\begin{array}{lll}1.79 & 0.215 & -3.27\end{array}$

1.94

$0.0645 \quad 2.36$

1.12

$0.183 \quad 2.57$

$\begin{array}{lll}0.339 & 0.0287 & -3.03\end{array}$

0.34

$0.23 \quad 5.01$

$\begin{array}{lll}0.513 & 0.0622 & -0.78\end{array}$

$\begin{array}{lll}2.46 & 0.222 \quad-3.38\end{array}$

0.55

2.51

$0.025 \quad-2.79$

$\begin{array}{lll}1.83 & 0.233 \quad-0.82\end{array}$

$0.334 \quad 0.0268 \quad-4.63$

$0.0511-1.76$

$\begin{array}{lll}2.45 & 0.212 \quad-3.90\end{array}$

$\begin{array}{lll}1.61 & 0.129 & -5.57\end{array}$

$\begin{array}{lll}1.74 & 0.123 & 2.51\end{array}$

$\begin{array}{lll}0.549 & 0.0434 & -1.89\end{array}$

$\begin{array}{lll}0.255 & 0.0265 & 6.17\end{array}$

$0.246 \quad 0.0244 \quad 2.31$

$\begin{array}{lll}1.65 & 0.136 & -3.08\end{array}$

$\begin{array}{lll}1.69 & 0.173 \quad 3.10\end{array}$

1.64

$0.158 \quad 0.20$

$\begin{array}{lll}0.246 & 0.0261 \quad 2.32\end{array}$

$0.247 \quad 0.035 \quad-0.28$

$\begin{array}{lll}0.25 & 0.0298 & 0.66\end{array}$

1.63

$0.175 \quad-0.69$

0.545

$0.0812 \quad-4.43$

0.554

$0.0816 \quad-2.81$

$\begin{array}{lll}0.56 & 0.0858 & -1.75\end{array}$

0.244

$0.0317 \quad-1.51$

0.0401

$\begin{array}{lll}0.042 & 0.013 & 16.57\end{array}$

$\begin{array}{lll}0.0391 \quad 0.0112 & 8.51\end{array}$

0.552

$0.0832 \quad-3.08$

$\begin{array}{lllllll}3.79 & 0.193 & 2.31 & 3.61 & 0.132 & -2.46\end{array}$

$\begin{array}{lll}3.8 & 0.236 \quad 2.80\end{array}$

$0.0404 \quad 0.0121 \quad 12.23$

0.0285

$0.0099 \quad-5.07 \quad 0.0293$

$0.0101 \quad-2.33$

0.0309

$0.0113 \quad 3.03$

$0.0296 \quad 0.0104 \quad-1.43$

0.0168

$0.0062 \quad-26.95$

$\begin{array}{lll}0.0168 & 0.0063 & -26.92\end{array}$

0.0167

$0.0058 \quad-27.47$

0.0168 
Chapter 2.1 LA-ICP-MS Transient Signal Quantification of NIST, MPI-DING, USGS and CGSG Glass Reference Materials by Ratioing, Standardization, and Normalization (RSN)

\section{Table 31}

Element concentrations of BCR-2G obtained from RSN strategy with NIST 610, StHs6/80-G and GSD-1G as external reference materials. Values are in units of $\mu \mathrm{g} / \mathrm{g}$ except for major elements which are specified in weight percent $(\% \mathrm{~m} / \mathrm{m})$. Standard deviation $(1 \mathrm{~s})$ stems from 89 measurements

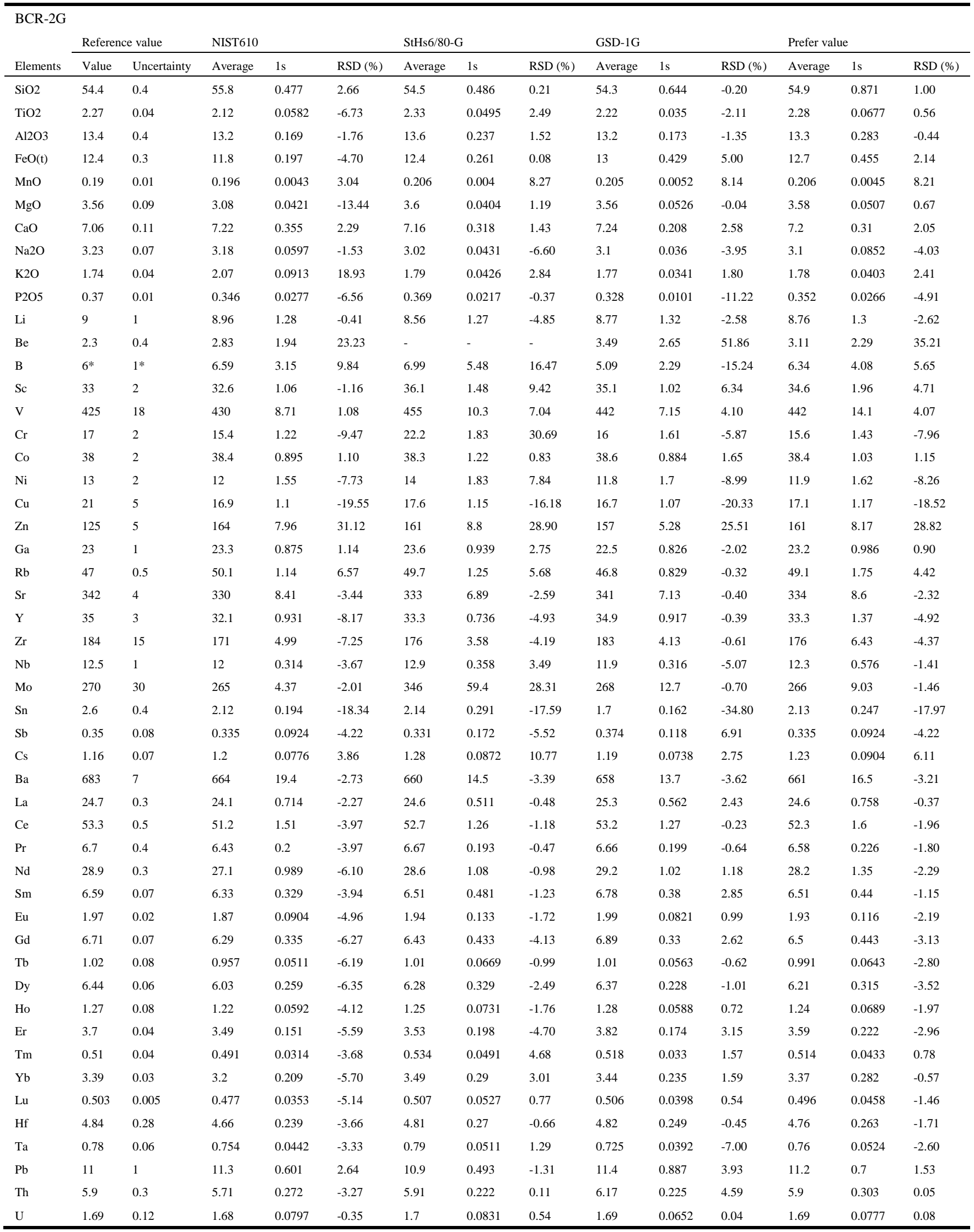


Chapter 2.1 LA-ICP-MS Transient Signal Quantification of NIST, MPI-DING, USGS and CGSG Glass Reference Materials by Ratioing, Standardization, and Normalization (RSN)

\section{Table $3 \mathrm{~m}$}

Element concentrations of BHVO-2G obtained from RSN strategy with NIST 610, StHs6/80-G and GSD-1G as external reference materials. Values are

in units of $\mu \mathrm{g} / \mathrm{g}$ except for major elements which are specified in weight percent $(\% \mathrm{~m} / \mathrm{m})$. Standard deviation $(1 \mathrm{~s})$ stems from 75 measurements.

\begin{tabular}{|c|c|c|c|c|c|c|c|c|c|c|c|c|c|c|}
\hline \multicolumn{15}{|c|}{ BHVO-2G } \\
\hline \multirow[b]{2}{*}{ Elements } & \multicolumn{2}{|c|}{ Reference value } & \multicolumn{3}{|l|}{ NIST610 } & \multicolumn{3}{|c|}{ StHs6/80-G } & \multicolumn{3}{|l|}{ GSD-1G } & \multicolumn{3}{|c|}{ Prefer value } \\
\hline & Value & Uncertainty & Average & $1 \mathrm{~s}$ & RSD (\%) & Average & $1 \mathrm{~s}$ & $\operatorname{RSD}(\%)$ & Average & $1 \mathrm{~s}$ & $\operatorname{RSD}(\%)$ & Average & $1 \mathrm{~s}$ & $\operatorname{RSD}(\%)$ \\
\hline $\mathrm{TiO} 2$ & $2.79 *$ & $0.02^{*}$ & 2.55 & 0.0553 & -8.58 & 2.79 & 0.0618 & 0.06 & 2.66 & 0.0349 & -4.63 & 2.73 & 0.0835 & -2.10 \\
\hline $\mathrm{Al} 2 \mathrm{O} 3$ & $13.6^{*}$ & $0.1^{*}$ & 13.2 & 0.172 & -2.94 & 13.5 & 0.222 & -0.59 & 13.1 & 0.156 & -3.65 & 13.3 & 0.258 & -2.31 \\
\hline $\mathrm{FeO}(\mathrm{t})$ & $11.3^{*}$ & $0.1^{*}$ & 10.7 & 0.231 & -5.39 & 11.1 & 0.202 & -1.84 & 11.7 & 0.345 & 3.13 & 11.3 & 0.361 & 0.27 \\
\hline $\mathrm{MnO}$ & $0.17^{*}$ & $0.03^{*}$ & 0.169 & 0.0037 & -0.79 & 0.176 & 0.0029 & 3.74 & 0.176 & 0.0039 & 3.61 & 0.176 & 0.0031 & 3.58 \\
\hline $\mathrm{MgO}$ & $7.13^{*}$ & $0.02^{*}$ & 6.29 & 0.0988 & -11.72 & 7.32 & 0.089 & 2.63 & 7.23 & 0.138 & 1.35 & 7.27 & 0.121 & 2.00 \\
\hline $\mathrm{CaO}$ & $11.4^{*}$ & $0.1^{*}$ & 11.2 & 0.331 & -1.55 & 11.1 & 0.19 & -2.70 & 11.2 & 0.471 & -1.93 & 11.2 & 0.347 & -2.10 \\
\hline $\mathrm{Na} 2 \mathrm{O}$ & $2.4^{*}$ & $0.1^{*}$ & 2.28 & 0.0698 & -4.92 & 2.15 & 0.0557 & -10.49 & 2.21 & 0.0488 & -7.85 & 2.21 & 0.0818 & -7.73 \\
\hline $\mathrm{K} 2 \mathrm{O}$ & $0.51^{*}$ & $0.02 *$ & 0.584 & 0.0237 & 14.51 & 0.507 & 0.0149 & -0.49 & 0.505 & 0.0135 & -1.03 & 0.506 & 0.0143 & -0.69 \\
\hline $\mathrm{P} 2 \mathrm{O} 5$ & $0.29^{*}$ & $0.02^{*}$ & 0.267 & 0.0226 & -8.02 & 0.28 & 0.0156 & -3.35 & 0.249 & 0.0055 & -14.20 & 0.266 & 0.0199 & -8.30 \\
\hline $\mathrm{Li}$ & $4.4^{*}$ & $0.8^{*}$ & 4.59 & 1.42 & 4.31 & 4.5 & 1.45 & 2.32 & 4.46 & 1.42 & 1.26 & 4.51 & 1.44 & 2.54 \\
\hline B & - & - & - & - & - & 5.91 & - & - & - & - & - & - & - & - \\
\hline $\mathrm{Sc}$ & 33 & 2 & 30.9 & 0.965 & -6.23 & 34.5 & 1.41 & 4.60 & 33.3 & 0.891 & 0.77 & 32.9 & 1.88 & -0.39 \\
\hline $\mathrm{V}$ & 308 & 19 & 325 & 5.47 & 5.48 & 344 & 8.74 & 11.60 & 332 & 6.48 & 7.85 & 334 & 10.7 & 8.31 \\
\hline $\mathrm{Cr}$ & $293^{*}$ & $12 *$ & 286 & 7.28 & -2.23 & 415 & 28.7 & 41.60 & 296 & 17.9 & 1.19 & 290 & 12.5 & -0.94 \\
\hline Co & 44 & 2 & 46.5 & 1.19 & 5.61 & 46 & 1.49 & 4.63 & 46.6 & 1.02 & 5.80 & 46.3 & 1.28 & 5.29 \\
\hline $\mathrm{Ni}$ & 116 & 7 & 125 & 3.52 & 7.98 & 145 & 8.29 & 25.37 & 123 & 3.99 & 6.44 & 124 & 3.87 & 7.25 \\
\hline $\mathrm{Cu}$ & 127 & 11 & 121 & 2.64 & -5.11 & 125 & 5.02 & -1.77 & 119 & 4.5 & -6.47 & 121 & 4.76 & -4.43 \\
\hline $\mathrm{Zn}$ & 102 & 6 & 128 & 6.75 & 25.75 & 126 & 5.58 & 23.10 & 122 & 3.92 & 19.29 & 125 & 6.23 & 22.89 \\
\hline $\mathrm{Ga}$ & 22 & 3 & 22.9 & 1.2 & 4.11 & 23 & 1.24 & 4.66 & 22.3 & 1.06 & 1.18 & 22.7 & 1.18 & 3.32 \\
\hline $\mathrm{Rb}$ & 9.2 & 0.04 & 9.96 & 0.329 & 8.31 & 9.83 & 0.381 & 6.88 & 9.3 & 0.27 & 1.12 & 9.72 & 0.433 & 5.70 \\
\hline $\mathrm{Sr}$ & 396 & 1 & 384 & 9.33 & -3.12 & 386 & 7.69 & -2.54 & 393 & 7.45 & -0.67 & 387 & 8.92 & -2.24 \\
\hline $\mathrm{Y}$ & 26 & 2 & 23.2 & 0.827 & -10.62 & 24 & 0.733 & -7.53 & 25.1 & 0.631 & -3.33 & 24.1 & 1.04 & -7.45 \\
\hline $\mathrm{Zr}$ & 170 & 7 & 156 & 5.03 & -8.49 & 161 & 3.41 & -5.54 & 166 & 3.37 & -2.43 & 160 & 5.66 & -5.72 \\
\hline Mo & $3.8^{*}$ & $0.2^{*}$ & 4.27 & 0.293 & 12.28 & 5.34 & 0.876 & 40.48 & 4.32 & 0.349 & 13.67 & 4.28 & 0.317 & 12.73 \\
\hline Sn & $2.6^{*}$ & $0.6^{*}$ & 1.7 & 0.151 & -34.70 & 1.73 & 0.255 & -33.65 & 1.36 & 0.125 & -47.71 & 1.71 & 0.21 & -34.17 \\
\hline $\mathrm{Sb}$ & $0.3^{*}$ & $0.13^{*}$ & - & - & - & - & - & - & - & - & - & - & - & - \\
\hline $\mathrm{Cs}$ & 0.1 & 0.02 & - & - & - & - & - & - & - & - & - & - & - & - \\
\hline $\mathrm{Ba}$ & 131 & 2 & 128 & 3.29 & -2.34 & 127 & 2.92 & -3.29 & 126 & 2.78 & -3.74 & 127 & 3.12 & -3.09 \\
\hline $\mathrm{La}$ & 15.2 & 0.2 & 14.7 & 0.428 & -3.43 & 14.9 & 0.393 & -1.97 & 15.3 & 0.373 & 0.64 & 14.9 & 0.46 & -1.77 \\
\hline $\mathrm{Ce}$ & 37.6 & 0.2 & 36.2 & 0.88 & -3.66 & 37 & 0.961 & -1.56 & 37.4 & 0.83 & -0.65 & 36.8 & 0.984 & -2.09 \\
\hline $\operatorname{Pr}$ & 5.35 & 0.22 & 5.01 & 0.157 & -6.36 & 5.21 & 0.22 & -2.55 & 5.16 & 0.125 & -3.47 & 5.13 & 0.196 & -4.19 \\
\hline $\mathrm{Nd}$ & 24.5 & 0.2 & 23.1 & 1.04 & -5.69 & 24.3 & 0.975 & -0.89 & 24.7 & 1.03 & 1.02 & 24 & 1.19 & -2.13 \\
\hline $\mathrm{Sm}$ & 6.1 & 0.03 & 5.77 & 0.363 & -5.45 & 5.85 & 0.506 & -4.11 & 6.15 & 0.366 & 0.89 & 5.9 & 0.434 & -3.27 \\
\hline $\mathrm{Eu}$ & 2.07 & 0.01 & 1.95 & 0.12 & -5.83 & 1.99 & 0.156 & -3.95 & 2.05 & 0.126 & -0.86 & 1.99 & 0.141 & -3.76 \\
\hline $\mathrm{Gd}$ & 6.16 & 0.05 & 5.85 & 0.425 & -5.03 & 6.03 & 0.464 & -2.07 & 6.44 & 0.387 & 4.52 & 6.09 & 0.493 & -1.15 \\
\hline $\mathrm{Tb}$ & 0.92 & 0.04 & 0.843 & 0.0487 & -8.42 & 0.895 & 0.0708 & -2.69 & 0.893 & 0.0441 & -2.89 & 0.876 & 0.0617 & -4.80 \\
\hline Dy & 5.28 & 0.05 & 5 & 0.266 & -5.27 & 5.21 & 0.334 & -1.39 & 5.28 & 0.239 & 0.04 & 5.16 & 0.309 & -2.36 \\
\hline Но & 0.98 & 0.04 & 0.914 & 0.0598 & -6.77 & 0.937 & 0.069 & -4.42 & 0.963 & 0.0571 & -1.73 & 0.935 & 0.065 & -4.54 \\
\hline Er & 2.56 & 0.02 & 2.39 & 0.16 & -6.66 & 2.4 & 0.166 & -6.42 & 2.61 & 0.166 & 1.97 & 2.45 & 0.189 & -4.12 \\
\hline $\mathrm{Tm}$ & 0.34 & 0.02 & 0.301 & 0.0277 & -11.33 & 0.324 & 0.0347 & -4.65 & 0.318 & 0.0267 & -6.34 & 0.314 & 0.0317 & -7.55 \\
\hline $\mathrm{Yb}$ & 2.01 & 0.02 & 1.84 & 0.171 & -8.60 & 2 & 0.216 & -0.63 & 1.98 & 0.173 & -1.73 & 1.93 & 0.202 & -3.76 \\
\hline $\mathrm{Lu}$ & 0.279 & 0.003 & 0.258 & 0.0299 & -7.58 & 0.275 & 0.0344 & -1.31 & 0.274 & 0.0304 & -1.82 & 0.269 & 0.0328 & -3.57 \\
\hline $\mathrm{Hf}$ & 4.32 & 0.18 & 4.16 & 0.272 & -3.75 & 4.27 & 0.236 & -1.08 & 4.27 & 0.257 & -1.12 & 4.23 & 0.262 & -2.03 \\
\hline $\mathrm{Ta}$ & 1.15 & 0.1 & 1.09 & 0.0609 & -4.87 & 1.15 & 0.0786 & 0.21 & 1.05 & 0.0514 & -9.06 & 1.1 & 0.0784 & -4.31 \\
\hline $\mathrm{Pb}$ & 1.7 & 0.2 & 1.94 & 0.162 & 14.16 & 1.85 & 0.146 & 9.04 & 1.95 & 0.213 & 14.99 & 1.91 & 0.171 & 12.29 \\
\hline Th & 1.22 & 0.05 & 1.15 & 0.0679 & -5.43 & 1.19 & 0.0579 & -2.05 & 1.24 & 0.0672 & 1.72 & 1.19 & 0.0721 & -2.22 \\
\hline $\mathrm{U}$ & 0.403 & 0.003 & 0.419 & 0.0272 & 3.97 & 0.427 & 0.0313 & 5.97 & 0.418 & 0.0276 & 3.71 & 0.421 & 0.0291 & 4.53 \\
\hline
\end{tabular}


Chapter 2.1 LA-ICP-MS Transient Signal Quantification of NIST, MPI-DING, USGS and CGSG Glass Reference Materials by Ratioing, Standardization, and Normalization (RSN)

\section{Table 3n}

Element concentrations of GSD-1G obtained from RSN strategy with NIST 610, StHs6/80-G and GSD-1G as external reference materials. Values are in units of $\mu \mathrm{g} / \mathrm{g}$ except for major elements which are specified in weight percent $(\% \mathrm{~m} / \mathrm{m})$. Standard deviation $(1 \mathrm{~s})$ stems from $120 \mathrm{measurements}$.

\begin{tabular}{|c|c|c|c|c|c|c|c|c|c|c|c|c|c|c|}
\hline \multirow[b]{2}{*}{ Elements } & Referen & value & NIST610 & & \multirow[b]{2}{*}{ RSD (\%) } & \multicolumn{3}{|c|}{ StHs6/80-G } & GSD-1G & & & Prefer val & & \\
\hline & Value & Uncertainty & Average & $1 \mathrm{~s}$ & & Average & $1 \mathrm{~s}$ & $\operatorname{RSD}(\%)$ & Average & $1 \mathrm{~s}$ & $\operatorname{RSD}(\%)$ & Average & $1 \mathrm{~s}$ & $\operatorname{RSD}(\%)$ \\
\hline $\mathrm{TiO} 2$ & 1.24 & 0.06 & 1.18 & 0.0297 & -5.20 & 1.3 & 0.021 & 4.72 & 1.24 & 0.0103 & 0.24 & 1.27 & 0.0324 & 2.59 \\
\hline $\mathrm{Al} 2 \mathrm{O} 3$ & 13.4 & 0.3 & 13.3 & 0.219 & -0.66 & 13.8 & 0.17 & 3.22 & 13.4 & 0.0792 & 0.15 & 13.5 & 0.282 & 0.93 \\
\hline $\mathrm{FeO}(\mathrm{t})$ & 13.3 & 0.1 & 12 & 0.407 & -9.51 & 12.7 & 0.216 & -4.43 & 13.3 & 0.073 & 0.28 & 13 & 0.353 & -2.19 \\
\hline $\mathrm{MnO}$ & 0.0284 & 0.0026 & 0.0269 & 0.0006 & -5.28 & 0.0284 & 0.0004 & 0.03 & 0.0285 & 0.0002 & 0.17 & 0.0284 & 0.0003 & 0.10 \\
\hline $\mathrm{MgO}$ & 3.6 & 0.04 & 3.1 & 0.0637 & -13.79 & 3.65 & 0.0593 & 1.51 & 3.61 & 0.0271 & 0.27 & 3.63 & 0.0519 & 0.92 \\
\hline $\mathrm{CaO}$ & 7.2 & 0.1 & 7.2 & 0.385 & 0.03 & 7.23 & 0.39 & 0.48 & 7.21 & 0.226 & 0.17 & 7.22 & 0.346 & 0.23 \\
\hline $\mathrm{Na} 2 \mathrm{O}$ & 3.6 & 0.2 & 3.7 & 0.0687 & 2.79 & 3.51 & 0.0408 & -2.39 & 3.61 & 0.03 & 0.29 & 3.61 & 0.0919 & 0.23 \\
\hline $\mathrm{K} 2 \mathrm{O}$ & 3 & 0.1 & 3.57 & 0.135 & 18.88 & 3.14 & 0.124 & 4.68 & 3.05 & 0.0204 & 1.81 & 3.1 & 0.101 & 3.31 \\
\hline $\mathrm{P} 2 \mathrm{O} 5$ & 0.197 & 0.0367 & 0.214 & 0.0157 & 8.65 & 0.229 & 0.0083 & 15.97 & 0.198 & 0.0033 & 0.29 & 0.214 & 0.0167 & 8.51 \\
\hline $\mathrm{Li}$ & 43 & 6 & 43.4 & 2.21 & 1.00 & 43.3 & 3.3 & 0.74 & 43.2 & 1.41 & 0.45 & 43.3 & 2.46 & 0.74 \\
\hline B & 50 & 20 & 65.4 & 7.64 & 30.90 & 69.5 & 42.9 & 38.91 & 50.3 & 3.36 & 0.57 & 62.1 & 26.9 & 24.18 \\
\hline $\mathrm{Sc}$ & 52 & 2 & 48.2 & 1.58 & -7.31 & 53 & 3.06 & 1.90 & 52.1 & 0.616 & 0.10 & 51 & 2.93 & -1.83 \\
\hline $\mathrm{V}$ & 44 & 2 & 42.2 & 1.01 & -4.13 & 45.1 & 1.02 & 2.53 & 44.1 & 0.653 & 0.12 & 43.8 & 1.53 & -0.51 \\
\hline $\mathrm{Cr}$ & 42 & 3 & 40.8 & 2.25 & -2.85 & 59.5 & 4.63 & 41.62 & 42.1 & 1.55 & 0.35 & 41.4 & 2.06 & -1.32 \\
\hline Co & 40 & 2 & 39.4 & 0.922 & -1.59 & 39.5 & 0.989 & -1.28 & 40.1 & 0.377 & 0.20 & 39.6 & 0.876 & -0.93 \\
\hline $\mathrm{Ni}$ & 58 & 4 & 58.1 & 2.02 & 0.20 & 68.4 & 4.86 & 17.89 & 58.2 & 1.56 & 0.36 & 58.2 & 1.81 & 0.27 \\
\hline $\mathrm{Cu}$ & 42 & 2 & 42.3 & 1.58 & 0.70 & 43.9 & 1.79 & 4.50 & 42.1 & 0.927 & 0.32 & 42.8 & 1.69 & 1.89 \\
\hline $\mathrm{Zn}$ & 54 & 2 & 57 & 3.51 & 5.54 & 55.6 & 3.03 & 3.00 & 54.1 & 2.09 & 0.19 & 55.6 & 3.18 & 3.00 \\
\hline $\mathrm{Ga}$ & 54 & 7 & 55.6 & 1.62 & 3.00 & 57 & 3.11 & 5.52 & 54.2 & 0.8 & 0.29 & 55.6 & 2.39 & 3.02 \\
\hline $\mathrm{Rb}$ & 37.3 & 0.4 & 39.9 & 1.02 & 6.99 & 39.7 & 1.03 & 6.45 & 37.3 & 0.475 & 0.12 & 39 & 1.45 & 4.66 \\
\hline $\mathrm{Sr}$ & 69.4 & 0.7 & 67 & 1.77 & -3.50 & 67.8 & 1.16 & -2.25 & 69.6 & 0.773 & 0.28 & 68.1 & 1.7 & -1.89 \\
\hline $\mathrm{Y}$ & 42 & 2 & 38.7 & 1.41 & -7.92 & 39.9 & 1.08 & -4.88 & 42.1 & 0.466 & 0.17 & 40.2 & 1.75 & -4.35 \\
\hline Mo & 39 & 3 & 38.3 & 1.52 & -1.68 & 49.1 & 8.82 & 25.96 & 39.1 & 0.624 & 0.36 & 38.7 & 1.25 & -0.71 \\
\hline $\mathrm{Sn}$ & 29 & 6 & 35.9 & 1.18 & 23.69 & 37 & 4.85 & 27.55 & 29.1 & 0.545 & 0.34 & 36.4 & 3.57 & 25.62 \\
\hline $\mathrm{Sb}$ & 43 & 7 & 38.4 & 1.44 & -10.61 & 33.8 & 28.6 & -21.47 & 43.1 & 0.721 & 0.17 & 38.4 & 1.44 & -10.61 \\
\hline Cs & 32 & 2 & 32.5 & 0.816 & 1.45 & 34.8 & 1.56 & 8.83 & 32.1 & 0.349 & 0.24 & 33.2 & 1.61 & 3.61 \\
\hline $\mathrm{Ba}$ & 67 & 1 & 67 & 1.94 & 0.04 & 66.9 & 1.48 & -0.13 & 67.1 & 0.763 & 0.22 & 67 & 1.49 & 0.04 \\
\hline $\mathrm{La}$ & 39.1 & 0.4 & 37.2 & 1.11 & -4.98 & 37.8 & 0.859 & -3.29 & 39.2 & 0.384 & 0.17 & 38 & 1.19 & -2.79 \\
\hline $\mathrm{Ce}$ & 41.4 & 0.4 & 39.6 & 1.05 & -4.24 & 41 & 0.84 & -0.95 & 41.5 & 0.383 & 0.19 & 40.7 & 1.13 & -1.72 \\
\hline $\operatorname{Pr}$ & 45 & 1 & 43.3 & 1.14 & -3.86 & 45 & 1.18 & -0.07 & 45.1 & 0.437 & 0.21 & 44.4 & 1.3 & -1.29 \\
\hline $\mathrm{Nd}$ & 44.7 & 0.5 & 41.6 & 1.44 & -6.88 & 43.5 & 1.83 & -2.66 & 44.9 & 1.11 & 0.35 & 43.3 & 1.99 & -3.17 \\
\hline $\mathrm{Sm}$ & 47.8 & 0.5 & 44.7 & 1.38 & -6.43 & 46.2 & 2.91 & -3.26 & 47.9 & 0.805 & 0.26 & 46.2 & 2.33 & -3.25 \\
\hline $\mathrm{Eu}$ & 41 & 2 & 38.5 & 1.07 & -6.07 & 39.9 & 2.56 & -2.66 & 41.1 & 0.43 & 0.14 & 39.8 & 1.94 & -2.96 \\
\hline $\mathrm{Gd}$ & 50.7 & 0.5 & 46.1 & 1.64 & -9.02 & 47.6 & 2.86 & -6.18 & 50.8 & 0.701 & 0.16 & 48.1 & 2.75 & -5.18 \\
\hline $\mathrm{Tb}$ & 47 & 2 & 44.4 & 1.29 & -5.60 & 46.2 & 2.85 & -1.66 & 47.1 & 0.512 & 0.18 & 45.9 & 2.18 & -2.44 \\
\hline Dy & 51.2 & 0.5 & 48.1 & 1.72 & -5.97 & 50.1 & 2.72 & -2.14 & 51.3 & 0.807 & 0.17 & 49.8 & 2.33 & -2.74 \\
\hline Ho & 49 & 2 & 46.4 & 1.49 & -5.39 & 47.3 & 2.9 & -3.53 & 49.1 & 0.43 & 0.22 & 47.5 & 2.23 & -3.00 \\
\hline $\mathrm{Er}$ & 40.1 & 0.4 & 36.7 & 1.25 & -8.40 & 37.1 & 2.21 & -7.40 & 40.2 & 0.524 & 0.20 & 37.9 & 2.14 & -5.37 \\
\hline $\mathrm{Tm}$ & 49 & 2 & 46.7 & 1.6 & -4.70 & 50.7 & 4.53 & 3.51 & 49.1 & 0.458 & 0.19 & 48.8 & 3.29 & -0.35 \\
\hline $\mathrm{Yb}$ & 50.9 & 0.5 & 47.4 & 1.46 & -6.90 & 51.1 & 4.98 & 0.42 & 51 & 0.693 & 0.24 & 49.8 & 3.53 & -2.15 \\
\hline $\mathrm{Lu}$ & 51.5 & 0.5 & 48.4 & 1.67 & -6.05 & 51.7 & 4.1 & 0.43 & 51.6 & 0.529 & 0.17 & 50.5 & 3.04 & -1.88 \\
\hline $\mathrm{Hf}$ & 39 & 2 & 37.9 & 1.34 & -2.91 & 39 & 1.95 & -0.07 & 39.1 & 0.579 & 0.24 & 38.6 & 1.53 & -0.95 \\
\hline $\mathrm{Ta}$ & 40 & 4 & 41.5 & 1.04 & 3.72 & 44 & 2.38 & 10.04 & 40.1 & 0.362 & 0.14 & 41.9 & 2.24 & 4.77 \\
\hline $\mathrm{Pb}$ & 50 & 2 & 49.5 & 1.65 & -1.10 & 47.3 & 1.92 & -5.33 & 50.1 & 0.617 & 0.25 & 48.9 & 1.93 & -2.13 \\
\hline Th & 41 & 2 & 38.2 & 1.43 & -6.78 & 39.6 & 1.72 & -3.51 & 41.1 & 0.4 & 0.21 & 39.6 & 1.77 & -3.47 \\
\hline $\mathrm{U}$ & 41 & 2 & 40.5 & 1.14 & -1.23 & 41.2 & 1.58 & 0.48 & 41.1 & 0.474 & 0.29 & 40.9 & 1.21 & -0.16 \\
\hline
\end{tabular}


Chapter 2.1 LA-ICP-MS Transient Signal Quantification of NIST, MPI-DING, USGS and CGSG Glass Reference Materials by Ratioing, Standardization, and Normalization (RSN)

\section{Table 3o}

Element concentrations of CGSG-1 obtained from RSN strategy with NIST 610, StHs6/80-G and GSD-1G as external reference materials. Values are in units of $\mu \mathrm{g} / \mathrm{g}$ except for major elements which are specified in weight percent $(\% \mathrm{~m} / \mathrm{m})$. Standard deviation $(1 \mathrm{~s})$ stems from 29 measurements

\begin{tabular}{|c|c|c|c|c|c|c|c|c|c|c|c|c|c|c|}
\hline \multirow[b]{2}{*}{ Elements } & \multicolumn{2}{|c|}{ Reference value } & \multicolumn{2}{|l|}{ NIST610 } & \multirow[b]{2}{*}{$\mathrm{RSD}(\%)$} & \multicolumn{3}{|c|}{ StHs6/80-G } & \multicolumn{3}{|l|}{ GSD-1G } & \multicolumn{3}{|c|}{ Prefer value } \\
\hline & Value & Uncertainty & Average & $1 \mathrm{~s}$ & & Average & $1 \mathrm{~s}$ & RSD (\%) & Average & $1 \mathrm{~s}$ & $\operatorname{RSD}(\%)$ & Average & $1 \mathrm{~s}$ & $\operatorname{RSD}(\%)$ \\
\hline $\mathrm{TiO} 2$ & 2.24 & 0.069 & 1.97 & 0.0413 & -12.20 & 2.22 & 0.032 & -1.12 & 2.15 & 0.0179 & -4.16 & 2.18 & 0.0428 & -2.64 \\
\hline $\mathrm{Al} 2 \mathrm{O} 3$ & 17.3 & 0.19 & 16.6 & 0.0993 & -4.01 & 17.3 & 0.0835 & -0.20 & 16.9 & 0.125 & -2.42 & 16.9 & 0.289 & -2.21 \\
\hline $\mathrm{FeO}(\mathrm{t})$ & 7.78 & 0.12 & 7.26 & 0.0835 & -6.68 & 7.73 & 0.171 & -0.69 & 8.16 & 0.246 & 4.89 & 7.95 & 0.303 & 2.10 \\
\hline $\mathrm{MnO}$ & 0.12 & 0.003 & 0.121 & 0.0026 & 0.66 & 0.13 & 0.0031 & 8.23 & 0.132 & 0.0024 & 10.14 & 0.131 & 0.003 & 9.19 \\
\hline $\mathrm{MgO}$ & 3.99 & 0.064 & 3.3 & 0.0354 & -17.42 & 3.95 & 0.0291 & -1.06 & 3.96 & 0.0608 & -0.69 & 3.96 & 0.0482 & -0.87 \\
\hline $\mathrm{CaO}$ & 5.83 & 0.067 & 6.15 & 0.202 & 5.42 & 6.2 & 0.141 & 6.39 & 6.4 & 0.131 & 9.79 & 6.25 & 0.194 & 7.20 \\
\hline $\mathrm{Na} 2 \mathrm{O}$ & 3.76 & 0.07 & 3.75 & 0.0873 & -0.37 & 3.61 & 0.0312 & -3.96 & 3.72 & 0.0308 & -1.02 & 3.69 & 0.0814 & -1.78 \\
\hline $\mathrm{K} 2 \mathrm{O}$ & 3.94 & 0.056 & 4.6 & 0.118 & 16.87 & 4.14 & 0.211 & 5.11 & 3.96 & 0.0517 & 0.51 & 4.05 & 0.178 & 2.81 \\
\hline $\mathrm{P} 2 \mathrm{O} 5$ & 1.12 & 0.032 & 1.14 & 0.047 & 2.09 & 1.23 & 0.0315 & 10.12 & 1.05 & 0.016 & -6.63 & 1.14 & 0.0971 & 1.75 \\
\hline $\mathrm{Li}$ & 24 & 1.85 & 24.3 & 1.65 & 1.21 & 26.6 & 3.43 & 10.77 & 24.8 & 1.77 & 3.29 & 25.2 & 2.62 & 5.09 \\
\hline B & $37.5^{*}$ & $3.66^{*}$ & 41.2 & 2.51 & 9.98 & 47.9 & 10.7 & 27.78 & 32.9 & 2.71 & -12.32 & 40.7 & 8.96 & 8.48 \\
\hline $\mathrm{Sc}$ & 12.4 & 0.66 & 11.7 & 0.529 & -5.81 & 12.7 & 0.665 & 2.46 & 13 & 0.604 & 5.11 & 12.5 & 0.833 & 0.58 \\
\hline $\mathrm{V}$ & 148 & 6.7 & 141 & 2.73 & -4.63 & 155 & 2.7 & 4.40 & 152 & 1.29 & 2.46 & 149 & 6.2 & 0.74 \\
\hline $\mathrm{Cr}$ & $34.2^{*}$ & $2.1^{*}$ & 30.4 & 1.88 & -11.19 & 44.8 & 4.15 & 30.95 & 32.1 & 2.01 & -6.03 & 31.3 & 2.14 & -8.61 \\
\hline Co & 24.2 & 1.52 & 23.9 & 0.705 & -1.39 & 24.3 & 0.828 & 0.21 & 24.6 & 0.745 & 1.76 & 24.2 & 0.822 & 0.19 \\
\hline $\mathrm{Ni}$ & 36.6 & 1.76 & 38.2 & 2.41 & 4.42 & 42.6 & 4.07 & 16.33 & 38.8 & 2.57 & 6.11 & 38.5 & 2.51 & 5.26 \\
\hline $\mathrm{Cu}$ & 19.2 & 4.43 & 18.8 & 0.739 & -2.21 & 19.7 & 1.08 & 2.55 & 19.3 & 1.17 & 0.65 & 19.3 & 1.08 & 0.33 \\
\hline $\mathrm{Zn}$ & 162 & 9 & 183 & 9.91 & 12.78 & 190 & 10.1 & 17.14 & 177 & 8.62 & 9.11 & 183 & 10.9 & 13.01 \\
\hline $\mathrm{Ga}$ & 26 & 1.49 & 29.4 & 2.27 & 12.96 & 30.6 & 2.62 & 17.79 & 29.1 & 2 & 11.73 & 29.7 & 2.41 & 14.16 \\
\hline $\mathrm{Rb}$ & 103 & 3.4 & 109 & 1.75 & 5.83 & 110 & 2.49 & 6.80 & 103 & 1.7 & 0.42 & 107 & 3.52 & 4.35 \\
\hline $\mathrm{Sr}$ & 1294 & 78.6 & 1256 & 18.3 & -2.96 & 1292 & 16.9 & -0.15 & 1349 & 23.7 & 4.27 & 1299 & 43.3 & 0.39 \\
\hline $\mathrm{Y}$ & 27.8 & 1.22 & 25.8 & 0.717 & -7.17 & 26.8 & 0.948 & -3.76 & 29.1 & 0.719 & 4.78 & 27.2 & 1.61 & -2.05 \\
\hline $\mathrm{Zr}$ & 558 & 18 & 498 & 8.66 & -10.69 & 518 & 15.1 & -7.17 & 561 & 13.1 & 0.45 & 526 & 28.8 & -5.80 \\
\hline Mo & 2.89 & 0.17 & 2.79 & 0.28 & -3.38 & 3.84 & 0.471 & 33.02 & 2.95 & 0.342 & 2.15 & 2.87 & 0.322 & -0.61 \\
\hline Sn & 6.82 & 0.78 & 7.15 & 0.412 & 4.84 & 7.03 & 0.461 & 3.08 & 6 & 0.389 & -12.05 & 7.09 & 0.441 & 3.96 \\
\hline $\mathrm{Sb}$ & 1.06 & 0.34 & 1.12 & 0.2 & 5.44 & 0.811 & 0.288 & -23.46 & 1.29 & 0.237 & 21.84 & 1.12 & 0.2 & 5.44 \\
\hline $\mathrm{Cs}$ & 0.94 & 0.04 & 0.935 & 0.064 & -0.48 & 1.03 & 0.0773 & 9.38 & 0.938 & 0.0603 & -0.18 & 0.967 & 0.0801 & 2.91 \\
\hline $\mathrm{Ba}$ & 2217 & 47 & 2119 & 44.3 & -4.40 & 2140 & 48 & -3.46 & 2147 & 35.3 & -3.17 & 2135 & 44.4 & -3.68 \\
\hline $\mathrm{La}$ & 171 & 5.5 & 156 & 1.91 & -8.65 & 161 & 3.13 & -5.71 & 171 & 1.95 & -0.28 & 163 & 6.4 & -4.88 \\
\hline $\mathrm{Ce}$ & 342 & 8.3 & 322 & 4.12 & -5.77 & 339 & 5.41 & -0.80 & 349 & 5.75 & 2.04 & 337 & 12.2 & -1.51 \\
\hline $\operatorname{Pr}$ & 35.7 & 0.86 & 33 & 0.555 & -7.58 & 34.5 & 0.68 & -3.47 & 35.3 & 0.451 & -1.14 & 34.3 & 1.11 & -4.06 \\
\hline $\mathrm{Nd}$ & 137 & 3.8 & 126 & 2.33 & -7.93 & 131 & 6.27 & -4.49 & 141 & 3.41 & 2.69 & 133 & 7.45 & -3.25 \\
\hline $\mathrm{Sm}$ & 18.8 & 0.51 & 17.2 & 0.771 & -8.43 & 17.6 & 0.999 & -6.55 & 19.2 & 0.856 & 2.22 & 18 & 1.24 & -4.25 \\
\hline $\mathrm{Eu}$ & 4.2 & 0.14 & 3.87 & 0.155 & -7.96 & 4.22 & 0.309 & 0.39 & 4.25 & 0.172 & 1.23 & 4.11 & 0.283 & -2.11 \\
\hline $\mathrm{Gd}$ & 11.9 & 1.38 & 10.6 & 0.562 & -10.98 & 11 & 0.599 & -7.46 & 12.1 & 0.657 & 1.35 & 11.2 & 0.866 & -5.70 \\
\hline $\mathrm{Tb}$ & 1.4 & 0.13 & 1.17 & 0.0644 & -16.27 & 1.16 & 0.0913 & -17.20 & 1.28 & 0.07 & -8.50 & 1.2 & 0.0937 & -13.99 \\
\hline Dy & 6.22 & 0.26 & 5.69 & 0.224 & -8.47 & 5.81 & 0.252 & -6.53 & 6.17 & 0.221 & -0.79 & 5.89 & 0.309 & -5.26 \\
\hline Но & 1 & 0.03 & 0.912 & 0.0554 & -8.77 & 0.91 & 0.0973 & -9.04 & 0.994 & 0.0612 & -0.64 & 0.938 & 0.0834 & -6.15 \\
\hline Er & 2.57 & 0.15 & 2.25 & 0.148 & -12.37 & 2.28 & 0.204 & -11.18 & 2.55 & 0.17 & -0.75 & 2.36 & 0.221 & -8.10 \\
\hline $\mathrm{Tm}$ & 0.33 & 0.03 & 0.275 & 0.0234 & -16.76 & 0.316 & 0.0562 & -4.11 & 0.296 & 0.0261 & -10.16 & 0.296 & 0.0419 & -10.34 \\
\hline $\mathrm{Yb}$ & 1.96 & 0.11 & 1.72 & 0.293 & -12.10 & 2.15 & 0.678 & 9.48 & 1.9 & 0.327 & -3.11 & 1.92 & 0.497 & -1.91 \\
\hline $\mathrm{Lu}$ & 0.27 & 0.015 & 0.232 & 0.0309 & -14.19 & 0.241 & 0.0352 & -10.61 & 0.252 & 0.0342 & -6.55 & 0.242 & 0.0345 & -10.45 \\
\hline $\mathrm{Hf}$ & 12 & 2.03 & 10.9 & 0.431 & -9.51 & 11 & 0.509 & -8.49 & 11.6 & 0.547 & -3.20 & 11.2 & 0.598 & -7.07 \\
\hline $\mathrm{Ta}$ & 2.67 & 0.13 & 2.42 & 0.115 & -9.24 & 2.61 & 0.224 & -2.30 & 2.41 & 0.106 & -9.68 & 2.48 & 0.182 & -7.07 \\
\hline $\mathrm{Pb}$ & 29.3 & 2.88 & 30.4 & 0.878 & 3.74 & 28.8 & 0.662 & -1.65 & 31.8 & 1.97 & 8.69 & 30.4 & 1.8 & 3.59 \\
\hline Th & 21.1 & 0.74 & 19.4 & 0.417 & -8.24 & 19.8 & 0.655 & -5.99 & 21.4 & 0.371 & 1.59 & 20.2 & 1.02 & -4.21 \\
\hline $\mathrm{U}$ & 3.43 & 0.17 & 3.5 & 0.138 & 2.00 & 3.62 & 0.136 & 5.44 & 3.64 & 0.0931 & 6.11 & 3.58 & 0.138 & 4.52 \\
\hline
\end{tabular}


Chapter 2.1 LA-ICP-MS Transient Signal Quantification of NIST, MPI-DING, USGS and CGSG Glass Reference Materials by Ratioing, Standardization, and Normalization (RSN)

\section{Table $3 p$}

Element concentrations of CGSG-2 obtained from RSN strategy with NIST 610, StHs6/80-G and GSD-1G as external reference materials. Values are in units of $\mu \mathrm{g} / \mathrm{g}$ except for major elements which are specified in weight percent $(\% \mathrm{~m} / \mathrm{m})$. Standard deviation $(1 \mathrm{~s})$ stems from 29 measurements

\begin{tabular}{|c|c|c|c|c|c|c|c|c|c|c|c|c|c|c|}
\hline \multicolumn{15}{|l|}{ CGSG-2 } \\
\hline \multirow[b]{2}{*}{ Elements } & \multicolumn{2}{|c|}{ Reference value } & \multicolumn{3}{|l|}{ NIST610 } & \multicolumn{3}{|c|}{ StHs6/80-G } & \multicolumn{3}{|l|}{ GSD-1G } & \multicolumn{3}{|c|}{ Prefer value } \\
\hline & Value & Uncertainty & Average & $1 \mathrm{~s}$ & RSD (\%) & Average & $1 \mathrm{~s}$ & $\operatorname{RSD}(\%)$ & Average & $1 \mathrm{~s}$ & $\operatorname{RSD}(\%)$ & Average & $1 \mathrm{~s}$ & $\operatorname{RSD}(\%)$ \\
\hline $\mathrm{TiO} 2$ & 0.59 & 0.041 & 0.525 & 0.01 & -10.97 & 0.599 & 0.0089 & 1.54 & 0.579 & 0.0067 & -1.83 & 0.589 & 0.0127 & -0.14 \\
\hline $\mathrm{Al} 2 \mathrm{O} 3$ & 20.77 & 0.31 & 19.9 & 0.155 & -4.15 & 21 & 0.175 & 1.25 & 20.6 & 0.202 & -0.60 & 20.5 & 0.498 & -1.17 \\
\hline $\mathrm{FeO}(\mathrm{t})$ & 6.76 & 0.13 & 6.31 & 0.0777 & -6.63 & 6.71 & 0.137 & -0.67 & 7.08 & 0.227 & 4.81 & 6.9 & 0.263 & 2.07 \\
\hline $\mathrm{MnO}$ & 0.13 & 0.005 & 0.124 & 0.0027 & -4.59 & 0.136 & 0.0032 & 4.28 & 0.137 & 0.0028 & 5.40 & 0.136 & 0.0031 & 4.84 \\
\hline $\mathrm{MgO}$ & 0.87 & 0.035 & 0.7 & 0.0094 & -19.57 & 0.849 & 0.0062 & -2.46 & 0.851 & 0.0163 & -2.14 & 0.85 & 0.0124 & -2.30 \\
\hline $\mathrm{CaO}$ & 1.7 & 0.053 & 1.99 & 0.148 & 16.95 & 2.05 & 0.115 & 20.68 & 2.1 & 0.13 & 23.27 & 2.05 & 0.139 & 20.30 \\
\hline $\mathrm{Na} 2 \mathrm{O}$ & 6.55 & 0.22 & 6.57 & 0.0984 & 0.32 & 6.4 & 0.0404 & -2.28 & 6.6 & 0.0692 & 0.73 & 6.52 & 0.114 & -0.41 \\
\hline $\mathrm{K} 2 \mathrm{O}$ & 6.95 & 0.15 & 7.99 & 0.171 & 14.99 & 7.28 & 0.392 & 4.80 & 6.93 & 0.0603 & -0.30 & 7.11 & 0.332 & 2.25 \\
\hline $\mathrm{P} 2 \mathrm{O} 5$ & 0.093 & 0.007 & 0.0925 & 0.0029 & -0.55 & 0.101 & 0.003 & 8.91 & 0.0854 & 0.0035 & -8.15 & 0.0934 & 0.0086 & 0.38 \\
\hline $\mathrm{Li}$ & 411 & 32.1 & 438 & 7.72 & 6.60 & 468 & 33.6 & 13.83 & 457 & 6.38 & 11.09 & 454 & 23.7 & 10.51 \\
\hline B & $614 *$ & $203^{*}$ & 676 & 20.5 & 10.12 & 830 & 205 & 35.17 & 565 & 27.8 & -7.93 & 690 & 162 & 12.45 \\
\hline $\mathrm{Sc}$ & 4.34 & 1.12 & 5.16 & 0.409 & 18.90 & 5.74 & 0.488 & 32.37 & 5.87 & 0.45 & 35.18 & 5.59 & 0.546 & 28.82 \\
\hline $\mathrm{V}$ & 206 & 9.21 & 200 & 3.85 & -3.00 & 221 & 4.14 & 7.34 & 217 & 2.57 & 5.48 & 213 & 9.94 & 3.27 \\
\hline $\mathrm{Cr}$ & $8.1^{*}$ & $1.7 *$ & 5.29 & 0.983 & -34.71 & 7.79 & 1.49 & -3.85 & 5.66 & 1.06 & -30.09 & 5.48 & 1.04 & -32.40 \\
\hline Co & 6.15 & 0.6 & 5.67 & 0.216 & -7.77 & 5.85 & 0.252 & -4.87 & 5.97 & 0.236 & -2.88 & 5.83 & 0.266 & -5.17 \\
\hline $\mathrm{Ni}$ & 4.48 & 0.66 & - & - & - & - & - & - & - & - & - & - & - & - \\
\hline $\mathrm{Cu}$ & 21.2 & 2.45 & 20.7 & 0.776 & -2.57 & 21.9 & 0.939 & 3.23 & 21.6 & 1.15 & 1.88 & 21.4 & 1.1 & 0.85 \\
\hline $\mathrm{Zn}$ & 124 & 10.3 & 146 & 7.26 & 18.05 & 151 & 8.57 & 22.02 & 140 & 6.29 & 12.87 & 146 & 8.76 & 17.65 \\
\hline $\mathrm{Ga}$ & 38 & 1.69 & 40 & 2.05 & 5.16 & 42.8 & 4.09 & 12.56 & 39.8 & 2.15 & 4.83 & 40.9 & 3.22 & 7.52 \\
\hline $\mathrm{Rb}$ & 124 & 10.09 & 129 & 2.39 & 4.07 & 132 & 2.65 & 6.49 & 124 & 1.58 & -0.06 & 128 & 4.04 & 3.50 \\
\hline $\mathrm{Sr}$ & 1161 & 76.87 & 1132 & 18.4 & -2.52 & 1180 & 13.3 & 1.64 & 1225 & 23.7 & 5.55 & 1179 & 42.7 & 1.56 \\
\hline $\mathrm{Y}$ & 28.5 & 1.44 & 25.6 & 0.618 & -10.26 & 27.2 & 0.586 & -4.70 & 29.5 & 0.619 & 3.47 & 27.4 & 1.72 & -3.83 \\
\hline $\mathrm{Zr}$ & 1424 & 71 & 1262 & 20.5 & -11.36 & 1352 & 28.5 & -5.03 & 1460 & 29 & 2.50 & 1358 & 84.9 & -4.63 \\
\hline Mo & 1.23 & 0.26 & 0.9 & 0.215 & -26.82 & 1.21 & 0.293 & -1.77 & 0.979 & 0.248 & -20.38 & 0.94 & 0.236 & -23.60 \\
\hline Sn & 11.1 & 1.18 & 10.9 & 0.449 & -1.38 & 11.4 & 0.872 & 2.49 & 9.28 & 0.53 & -16.43 & 11.2 & 0.726 & 0.56 \\
\hline $\mathrm{Sb}$ & 1.62 & 0.42 & 1.72 & 0.268 & 6.43 & 1.04 & 0.58 & -35.81 & 2 & 0.337 & 23.18 & 1.72 & 0.268 & 6.43 \\
\hline $\mathrm{Cs}$ & 2.02 & 0.14 & 1.91 & 0.0916 & -5.47 & 2.11 & 0.125 & 4.50 & 1.94 & 0.0897 & -4.00 & 1.99 & 0.136 & -1.66 \\
\hline $\mathrm{Ba}$ & 390 & 25.5 & 381 & 6.04 & -2.40 & 391 & 5.16 & 0.30 & 389 & 6.76 & -0.38 & 387 & 7.5 & -0.83 \\
\hline $\mathrm{La}$ & 160 & 6.65 & 147 & 1.96 & -8.29 & 154 & 3.06 & -3.68 & 163 & 1.73 & 2.07 & 155 & 7.17 & -3.30 \\
\hline $\mathrm{Ce}$ & 256 & 6.2 & 239 & 3.01 & -6.78 & 254 & 4.94 & -0.85 & 261 & 5.22 & 2.10 & 251 & 10.5 & -1.84 \\
\hline $\operatorname{Pr}$ & 23.2 & 0.8 & 21.2 & 0.401 & -8.73 & 22.5 & 0.405 & -2.95 & 23 & 0.402 & -0.98 & 22.2 & 0.863 & -4.22 \\
\hline $\mathrm{Nd}$ & 74.6 & 1.99 & 67.1 & 1.52 & -10.04 & 71.8 & 1.76 & -3.74 & 75.8 & 1.86 & 1.65 & 71.6 & 3.96 & -4.04 \\
\hline $\mathrm{Sm}$ & 9.75 & 0.24 & 9.09 & 0.391 & -6.78 & 9.37 & 0.514 & -3.88 & 10.3 & 0.451 & 5.34 & 9.58 & 0.679 & -1.78 \\
\hline $\mathrm{Eu}$ & 2.48 & 0.14 & 2.3 & 0.102 & -7.10 & 2.45 & 0.131 & -1.08 & 2.57 & 0.111 & 3.49 & 2.44 & 0.158 & -1.56 \\
\hline $\mathrm{Gd}$ & 6.86 & 0.94 & 6.08 & 0.446 & -11.43 & 6.4 & 0.474 & -6.73 & 7.13 & 0.518 & 3.96 & 6.54 & 0.652 & -4.73 \\
\hline $\mathrm{Tb}$ & 0.97 & 0.079 & 0.807 & 0.0524 & -16.84 & 0.842 & 0.0506 & -13.22 & 0.898 & 0.0577 & -7.44 & 0.849 & 0.0655 & -12.50 \\
\hline Dy & 4.96 & 0.23 & 4.41 & 0.214 & -11.05 & 4.72 & 0.237 & -4.85 & 4.97 & 0.229 & 0.16 & 4.7 & 0.321 & -5.25 \\
\hline Но & 0.9 & 0.03 & 0.825 & 0.0522 & -8.33 & 0.876 & 0.0926 & -2.72 & 0.92 & 0.0552 & 2.17 & 0.873 & 0.0792 & -2.96 \\
\hline Er & 2.66 & 0.1 & 2.4 & 0.176 & -9.72 & 2.66 & 0.444 & -0.05 & 2.78 & 0.212 & 4.69 & 2.61 & 0.341 & -1.69 \\
\hline $\mathrm{Tm}$ & 0.41 & 0.033 & 0.342 & 0.0358 & -16.63 & 0.393 & 0.0628 & -4.05 & 0.379 & 0.0388 & -7.65 & 0.371 & 0.0521 & -9.44 \\
\hline $\mathrm{Yb}$ & 2.76 & 0.18 & 2.38 & 0.188 & -13.63 & 2.56 & 0.469 & -7.21 & 2.7 & 0.215 & -2.34 & 2.55 & 0.342 & -7.73 \\
\hline $\mathrm{Lu}$ & 0.42 & 0.026 & 0.361 & 0.0349 & -14.14 & 0.391 & 0.0401 & -6.83 & 0.404 & 0.0394 & -3.73 & 0.385 & 0.0424 & -8.23 \\
\hline $\mathrm{Hf}$ & 35.4 & 2.47 & 32.7 & 0.871 & -7.53 & 33.3 & 1.86 & -5.80 & 35.5 & 0.88 & 0.35 & 33.9 & 1.76 & -4.33 \\
\hline $\mathrm{Ta}$ & 2.08 & 0.11 & 1.9 & 0.0768 & -8.72 & 2.08 & 0.24 & -0.13 & 1.92 & 0.0766 & -7.81 & 1.96 & 0.172 & -5.55 \\
\hline $\mathrm{Pb}$ & 129 & 10.77 & 141 & 4.53 & 9.23 & 137 & 3.28 & 6.22 & 149 & 10.6 & 15.62 & 142 & 8.55 & 10.36 \\
\hline Th & 75.3 & 2.41 & 68.8 & 1.54 & -8.65 & 72.2 & 2.95 & -4.08 & 77.4 & 1.11 & 2.84 & 72.8 & 4.09 & -3.30 \\
\hline $\mathrm{U}$ & 13.7 & 0.66 & 14.4 & 0.418 & 5.36 & 15.1 & 0.641 & 9.95 & 15.2 & 0.345 & 10.78 & 14.9 & 0.584 & 8.69 \\
\hline
\end{tabular}


Chapter 2.1 LA-ICP-MS Transient Signal Quantification of NIST, MPI-DING, USGS and CGSG Glass Reference Materials by Ratioing, Standardization, and Normalization (RSN)

\section{Table $3 \mathrm{q}$}

Element concentrations of CGSG-4 obtained from RSN strategy with NIST 610, StHs6/80-G and GSD-1G as external reference materials. Values are in units of $\mu \mathrm{g} / \mathrm{g}$ except for major elements which are specified in weight percent $(\% \mathrm{~m} / \mathrm{m})$. Standard deviation $(1 \mathrm{~s})$ stems from $29 \mathrm{measurements}$.

\begin{tabular}{|c|c|c|c|c|c|c|c|c|c|c|c|c|c|c|}
\hline \multirow[b]{3}{*}{ Elements } & \multirow{2}{*}{\multicolumn{2}{|c|}{ Reference value }} & \multirow{2}{*}{\multicolumn{3}{|c|}{ NIST610 }} & \multirow{2}{*}{\multicolumn{3}{|c|}{ StHs6/80-G }} & \multirow{2}{*}{\multicolumn{3}{|c|}{ GSD-1G }} & \multirow{2}{*}{\multicolumn{3}{|c|}{ Prefer value }} \\
\hline & & & & & & & & & & & & & & \\
\hline & Value & Uncertainty & Average & $1 \mathrm{~s}$ & $\operatorname{RSD}(\%)$ & Average & $1 \mathrm{~s}$ & $\operatorname{RSD}(\%)$ & Average & $1 \mathrm{~s}$ & $\operatorname{RSD}(\%)$ & Average & $1 \mathrm{~s}$ & $\operatorname{RSD}(\%)$ \\
\hline $\mathrm{SiO} 2$ & 63.74 & 0.32 & 65.4 & 0.356 & 2.57 & 63.8 & 0.165 & 0.17 & 64 & 0.272 & 0.36 & 64.4 & 0.746 & 1.03 \\
\hline $\mathrm{TiO} 2$ & 0.614 & 0.046 & 0.554 & 0.0071 & -9.85 & 0.627 & 0.0097 & 2.19 & 0.603 & 0.0062 & -1.79 & 0.615 & 0.0147 & 0.20 \\
\hline $\mathrm{Al} 2 \mathrm{O} 3$ & 14.77 & 0.4 & 14.3 & 0.0524 & -3.35 & 15.1 & 0.172 & 2.45 & 14.8 & 0.165 & 0.35 & 14.7 & 0.381 & -0.18 \\
\hline $\mathrm{FeO}(\mathrm{t})$ & 4.61 & 0.12 & 4.4 & 0.083 & -4.55 & 4.67 & 0.0888 & 1.45 & 4.91 & 0.176 & 6.48 & 4.79 & 0.181 & 3.97 \\
\hline $\mathrm{MnO}$ & 0.109 & 0.004 & 0.108 & 0.0015 & -0.69 & 0.118 & 0.0023 & 8.12 & 0.119 & 0.0019 & 8.74 & 0.118 & 0.0021 & 8.43 \\
\hline $\mathrm{MgO}$ & 2.16 & 0.071 & 1.8 & 0.0152 & -16.44 & 2.18 & 0.0204 & 1.09 & 2.18 & 0.0313 & 1.00 & 2.18 & 0.0265 & 1.05 \\
\hline $\mathrm{CaO}$ & 6.96 & 0.18 & 6.94 & 0.238 & -0.31 & 7.19 & 0.111 & 3.31 & 7.26 & 0.207 & 4.35 & 7.13 & 0.238 & 2.45 \\
\hline $\mathrm{Na} 2 \mathrm{O}$ & 2.8 & 0.054 & 2.8 & 0.0481 & 0.02 & 2.71 & 0.0173 & -3.24 & 2.79 & 0.0204 & -0.45 & 2.77 & 0.0513 & -1.22 \\
\hline $\mathrm{K} 2 \mathrm{O}$ & 2.63 & 0.054 & 2.98 & 0.134 & 13.27 & 2.7 & 0.0935 & 2.62 & 2.56 & 0.0637 & -2.50 & 2.63 & 0.105 & 0.06 \\
\hline $\mathrm{P} 2 \mathrm{O} 5$ & 0.253 & 0.012 & 0.26 & 0.0103 & 2.59 & 0.279 & 0.008 & 10.21 & 0.236 & 0.0056 & -6.82 & 0.257 & 0.0226 & 1.70 \\
\hline $\mathrm{Li}$ & 1120 & 68.7 & 1184 & 10.3 & 5.75 & 1241 & 75.1 & 10.82 & 1234 & 17.4 & 10.19 & 1220 & 51.5 & 8.92 \\
\hline $\mathrm{Be}$ & 2.96 & 0.22 & 3.79 & 2.12 & 28.09 & - & - & - & 4.27 & 2.4 & 44.34 & 4.03 & 2.28 & 36.21 \\
\hline B & $1575^{*}$ & $518^{*}$ & 1946 & 70.9 & 23.55 & 1995 & 440 & 26.66 & 1697 & 71.7 & 7.72 & 1879 & 291 & 19.31 \\
\hline $\mathrm{Sc}$ & 10.6 & 0.87 & 10.3 & 0.368 & -2.54 & 11.7 & 0.455 & 9.95 & 11.8 & 0.45 & 11.63 & 11.3 & 0.794 & 6.35 \\
\hline V & 88.9 & 3.05 & 85.9 & 1.6 & -3.38 & 94.5 & 1.86 & 6.25 & 92.8 & 1.18 & 4.36 & 91 & 4.03 & 2.41 \\
\hline $\mathrm{Cr}$ & $70 *$ & $3.5^{*}$ & 62.7 & 2.24 & -10.37 & 90.9 & 4.16 & 29.83 & 65.5 & 3.31 & -6.39 & 64.1 & 3.15 & -8.38 \\
\hline Co & 12.7 & 0.71 & 12.2 & 0.26 & -4.24 & 12.6 & 0.6 & -0.42 & 12.8 & 0.333 & 0.79 & 12.5 & 0.504 & -1.29 \\
\hline $\mathrm{Ni}$ & 29.3 & 1.52 & 28.8 & 2.48 & -1.59 & 35.8 & 5.33 & 22.25 & 29.9 & 2.81 & 1.98 & 29.4 & 2.7 & 0.19 \\
\hline $\mathrm{Cu}$ & 44.3 & 4.52 & 41.9 & 1.18 & -5.52 & 44 & 1.48 & -0.61 & 43.2 & 2.09 & -2.51 & 43 & 1.86 & -2.88 \\
\hline $\mathrm{Zn}$ & 125 & 7.92 & 149 & 6.42 & 19.21 & 148 & 6.13 & 18.17 & 141 & 4.09 & 12.97 & 146 & 6.59 & 16.79 \\
\hline $\mathrm{Ga}$ & 18.5 & 1.09 & 18.3 & 0.955 & -0.81 & 19.9 & 1.56 & 7.79 & 18.4 & 0.781 & -0.57 & 18.9 & 1.37 & 2.14 \\
\hline $\mathrm{Rb}$ & 84.9 & 3.66 & 90.4 & 1.04 & 6.50 & 92.1 & 1.84 & 8.44 & 86.2 & 1.44 & 1.48 & 89.5 & 2.89 & 5.47 \\
\hline $\mathrm{Sr}$ & 386 & 12.74 & 371 & 5.69 & -3.79 & 389 & 3.64 & 0.78 & 404 & 5.7 & 4.61 & 388 & 14.2 & 0.53 \\
\hline $\mathrm{Y}$ & 24.1 & 0.95 & 22.5 & 0.473 & -6.85 & 23.9 & 0.575 & -0.92 & 25.9 & 0.545 & 7.33 & 24.1 & 1.5 & -0.15 \\
\hline $\mathrm{Zr}$ & 270 & 6.23 & 243 & 3.22 & -10.17 & 258 & 2.3 & -4.55 & 276 & 2.59 & 2.16 & 259 & 13.9 & -4.19 \\
\hline $\mathrm{Nb}$ & 14.9 & 0.88 & 14.3 & 0.323 & -4.11 & 15.6 & 0.566 & 4.75 & 14.8 & 0.335 & -0.77 & 14.9 & 0.689 & -0.04 \\
\hline Mo & 2.12 & 0.18 & 1.94 & 0.211 & -8.49 & 2.47 & 0.358 & 16.64 & 2.09 & 0.259 & -1.24 & 2.02 & 0.249 & -4.86 \\
\hline Sn & 10.5 & 1.68 & 10.9 & 0.541 & 4.13 & 11.9 & 1.6 & 13.77 & 9.16 & 0.617 & -12.73 & 11.4 & 1.29 & 8.95 \\
\hline $\mathrm{Sb}$ & 1.74 & 0.58 & 1.85 & 0.224 & 6.60 & 1.12 & 0.762 & -35.44 & 2.14 & 0.279 & 23.09 & 1.85 & 0.224 & 6.60 \\
\hline Cs & 4.87 & 0.23 & 4.79 & 0.134 & -1.60 & 5.31 & 0.216 & 9.02 & 4.84 & 0.134 & -0.54 & 4.98 & 0.286 & 2.29 \\
\hline $\mathrm{Ba}$ & 736 & 21.6 & 711 & 6.61 & -3.42 & 725 & 12.1 & -1.55 & 730 & 9.21 & -0.88 & 722 & 12.4 & -1.95 \\
\hline $\mathrm{La}$ & 41.43 & 1.12 & 38.6 & 0.447 & -6.71 & 40.6 & 0.82 & -1.97 & 42.9 & 0.47 & 3.49 & 40.7 & 1.83 & -1.73 \\
\hline $\mathrm{Ce}$ & 73.3 & 1.71 & 70.2 & 0.865 & -4.27 & 74.3 & 1.42 & 1.32 & 75.9 & 1.26 & 3.61 & 73.5 & 2.71 & 0.22 \\
\hline $\operatorname{Pr}$ & 8.19 & 0.26 & 7.61 & 0.171 & -7.04 & 8.13 & 0.175 & -0.69 & 8.26 & 0.165 & 0.88 & 8 & 0.328 & -2.28 \\
\hline $\mathrm{Nd}$ & 30.8 & 0.92 & 28.3 & 0.928 & -8.27 & 30.3 & 1.19 & -1.48 & 31.8 & 1.13 & 3.18 & 30.1 & 1.81 & -2.19 \\
\hline $\mathrm{Sm}$ & 5.49 & 0.18 & 5.03 & 0.321 & -8.29 & 5.31 & 0.362 & -3.27 & 5.66 & 0.371 & 3.13 & 5.34 & 0.435 & -2.81 \\
\hline $\mathrm{Eu}$ & 1.24 & 0.045 & 1.16 & 0.0928 & -6.23 & 1.2 & 0.0989 & -3.06 & 1.3 & 0.104 & 4.54 & 1.22 & 0.114 & -1.58 \\
\hline Gd & 4.67 & 0.29 & 4.29 & 0.283 & -8.12 & 4.58 & 0.31 & -1.97 & 5.07 & 0.344 & 8.54 & 4.65 & 0.449 & -0.52 \\
\hline $\mathrm{Tb}$ & 0.72 & 0.048 & 0.637 & 0.0363 & -11.53 & 0.688 & 0.0441 & -4.44 & 0.709 & 0.0402 & -1.54 & 0.678 & 0.0504 & -5.84 \\
\hline Dy & 4.22 & 0.12 & 3.76 & 0.307 & -10.94 & 4.09 & 0.368 & -3.16 & 4.3 & 0.334 & 1.88 & 4.05 & 0.404 & -4.07 \\
\hline Ho & 0.84 & 0.029 & 0.769 & 0.0457 & -8.40 & 0.897 & 0.103 & 6.83 & 0.859 & 0.0538 & 2.27 & 0.842 & 0.09 & 0.23 \\
\hline Er & 2.49 & 0.1 & 2.23 & 0.13 & -10.37 & 2.18 & 0.148 & -12.35 & 2.6 & 0.135 & 4.25 & 2.34 & 0.23 & -6.16 \\
\hline $\mathrm{Tm}$ & 0.38 & 0.032 & 0.321 & 0.029 & -15.56 & 0.362 & 0.0416 & -4.85 & 0.356 & 0.0314 & -6.27 & 0.346 & 0.0389 & -8.90 \\
\hline $\mathrm{Yb}$ & 2.44 & 0.08 & 2.16 & 0.243 & -11.57 & 2.19 & 0.307 & -10.22 & 2.46 & 0.284 & 0.78 & 2.27 & 0.31 & -7.00 \\
\hline $\mathrm{Lu}$ & 0.37 & 0.023 & 0.327 & 0.0271 & -11.74 & 0.371 & 0.0381 & 0.38 & 0.37 & 0.0302 & -0.11 & 0.356 & 0.0383 & -3.82 \\
\hline $\mathrm{Hf}$ & 6.72 & 0.59 & 6.43 & 0.287 & -4.25 & 6.76 & 0.405 & 0.55 & 6.99 & 0.35 & 4.04 & 6.73 & 0.419 & 0.11 \\
\hline $\mathrm{Ta}$ & 0.97 & 0.1 & 0.93 & 0.0502 & -4.15 & 1.05 & 0.0998 & 8.73 & 0.95 & 0.0559 & -2.09 & 0.978 & 0.0906 & 0.83 \\
\hline $\mathrm{Pb}$ & 44.8 & 3.32 & 48.9 & 1.37 & 9.11 & 47.6 & 1.21 & 6.30 & 51.3 & 3.44 & 14.45 & 49.3 & 2.71 & 9.95 \\
\hline Th & 12.8 & 0.57 & 11.8 & 0.295 & -8.15 & 12.5 & 0.594 & -2.20 & 13.3 & 0.248 & 3.80 & 12.5 & 0.747 & -2.18 \\
\hline $\mathrm{U}$ & 2.72 & 0.17 & 2.7 & 0.111 & -0.80 & 2.79 & 0.128 & 2.71 & 2.81 & 0.1 & 3.28 & 2.77 & 0.124 & 1.73 \\
\hline
\end{tabular}


Chapter 2.1 LA-ICP-MS Transient Signal Quantification of NIST, MPI-DING, USGS and CGSG Glass Reference Materials by Ratioing, Standardization, and Normalization (RSN)

Table 3r

Element concentrations of CGSG-5 obtained from RSN strategy with NIST 610, StHs6/80-G and GSD-1G as external reference materials. Values are in units of $\mu \mathrm{g} / \mathrm{g}$ except for major elements which are specified in weight percent $(\% \mathrm{~m} / \mathrm{m})$. Standard deviation $(1 \mathrm{~s})$ stems from $25 \mathrm{measurements}$

\begin{tabular}{|c|c|c|c|c|c|c|c|c|c|c|c|c|c|c|}
\hline \multicolumn{15}{|l|}{ CGSG-5 } \\
\hline \multirow[b]{2}{*}{ Elements } & \multicolumn{2}{|c|}{ Reference value } & \multicolumn{3}{|l|}{ NIST610 } & \multicolumn{3}{|c|}{ StHs6/80-G } & \multicolumn{3}{|l|}{ GSD-1G } & \multicolumn{3}{|c|}{ Prefer value } \\
\hline & Value & Uncertainty & Average & $1 \mathrm{~s}$ & $\operatorname{RSD}(\%)$ & Average & $1 \mathrm{~s}$ & $\operatorname{RSD}(\%)$ & Average & $1 \mathrm{~s}$ & $\operatorname{RSD}(\%)$ & Average & $1 \mathrm{~s}$ & $\operatorname{RSD}(\%)$ \\
\hline $\mathrm{TiO} 2$ & 0.503 & 0.031 & 0.463 & 0.0061 & -8.01 & 0.526 & 0.0086 & 4.57 & 0.507 & 0.0052 & 0.76 & 0.516 & 0.0119 & 2.66 \\
\hline $\mathrm{Al} 2 \mathrm{O} 3$ & 15.75 & 0.36 & 15.5 & 0.0783 & -1.33 & 16.5 & 0.145 & 4.82 & 16.2 & 0.192 & 2.58 & 16.1 & 0.426 & 2.02 \\
\hline $\mathrm{FeO}(\mathrm{t})$ & 4.45 & 0.12 & 4.22 & 0.0592 & -5.21 & 4.57 & 0.0857 & 2.61 & 4.81 & 0.185 & 7.88 & 4.69 & 0.186 & 5.24 \\
\hline $\mathrm{MnO}$ & 0.0878 & 0.004 & 0.0886 & 0.001 & 0.88 & 0.0955 & 0.0021 & 8.73 & 0.0966 & 0.0016 & 9.97 & 0.096 & 0.0019 & 9.35 \\
\hline $\mathrm{MgO}$ & 1.53 & 0.04 & 1.31 & 0.0157 & -14.17 & 1.59 & 0.0166 & 3.78 & 1.59 & 0.0273 & 3.80 & 1.59 & 0.0226 & 3.79 \\
\hline $\mathrm{CaO}$ & 4.73 & 0.1 & 4.97 & 0.223 & 5.00 & 5.09 & 0.105 & 7.59 & 5.18 & 0.237 & 9.59 & 5.08 & 0.217 & 7.39 \\
\hline $\mathrm{Na} 2 \mathrm{O}$ & 11.35 & 0.52 & 11.7 & 0.156 & 2.82 & 11.3 & 0.0506 & -0.70 & 11.5 & 0.065 & 1.67 & 11.5 & 0.195 & 1.27 \\
\hline $\mathrm{K} 2 \mathrm{O}$ & 1.93 & 0.033 & 2.24 & 0.0936 & 16.19 & 2.01 & 0.0634 & 4.03 & 1.92 & 0.0445 & -0.27 & 1.97 & 0.0687 & 1.88 \\
\hline $\mathrm{P} 2 \mathrm{O} 5$ & 0.21 & 0.01 & 0.215 & 0.0078 & 2.30 & 0.23 & 0.0054 & 9.54 & 0.194 & 0.0043 & -7.43 & 0.212 & 0.0185 & 1.05 \\
\hline $\mathrm{Li}$ & 1905 & 164 & 2094 & 30 & 9.91 & 2189 & 114 & 14.89 & 2182 & 39.8 & 14.52 & 2155 & 83.8 & 13.11 \\
\hline B & $4732 *$ & $2675^{*}$ & 5222 & 283 & 10.37 & 4617 & 789 & -2.44 & 4519 & 297 & -4.50 & 4786 & 601 & 1.14 \\
\hline $\mathrm{Sc}$ & 8.56 & 0.84 & 8.69 & 0.375 & 1.54 & 10 & 0.472 & 17.32 & 9.92 & 0.437 & 15.89 & 9.55 & 0.746 & 11.58 \\
\hline $\mathrm{V}$ & 101.2 & 3.81 & 101 & 1.55 & -0.21 & 111 & 2.63 & 9.43 & 108 & 1.82 & 7.13 & 107 & 4.64 & 5.45 \\
\hline $\mathrm{Cr}$ & $29.5^{*}$ & $2.2 *$ & 25.4 & 2.12 & -14.05 & 36.2 & 3 & 22.79 & 26.1 & 2.42 & -11.51 & 25.7 & 2.31 & -12.78 \\
\hline Co & 12.3 & 0.84 & 12.3 & 0.341 & -0.07 & 12.6 & 0.528 & 2.40 & 12.9 & 0.465 & 5.02 & 12.6 & 0.519 & 2.45 \\
\hline $\mathrm{Ni}$ & 15.8 & 1.61 & 16.7 & 2.38 & 5.49 & 22.4 & 5.46 & 41.67 & 17.2 & 2.62 & 8.66 & 16.9 & 2.51 & 7.08 \\
\hline $\mathrm{Cu}$ & 50.4 & 2.19 & 53.1 & 1.01 & 5.27 & 55.8 & 1.31 & 10.71 & 54 & 2.31 & 7.21 & 54.3 & 1.99 & 7.73 \\
\hline $\mathrm{Zn}$ & 79.5 & 5.85 & 92.1 & 4.88 & 15.83 & 90 & 4.86 & 13.15 & 88 & 3.52 & 10.65 & 90 & 4.77 & 13.21 \\
\hline $\mathrm{Ga}$ & 18.88 & 0.66 & 19.2 & 1.39 & 1.66 & 20.6 & 1.39 & 9.02 & 19.4 & 1.43 & 2.73 & 19.7 & 1.53 & 4.47 \\
\hline $\mathrm{Rb}$ & 37.8 & 2.2 & 40.4 & 0.813 & 6.84 & 41.2 & 1.24 & 9.00 & 38.3 & 1.17 & 1.34 & 40 & 1.64 & 5.73 \\
\hline $\mathrm{Sr}$ & 767 & 50.7 & 779 & 10.1 & 1.51 & 812 & 10.2 & 5.86 & 847 & 11.5 & 10.37 & 812 & 29.7 & 5.92 \\
\hline $\mathrm{Y}$ & 10.4 & 0.31 & 9.53 & 0.26 & -8.40 & 10.1 & 0.321 & -3.26 & 11 & 0.299 & 5.46 & 10.2 & 0.664 & -2.07 \\
\hline $\mathrm{Zr}$ & 181 & 4.89 & 172 & 2.39 & -4.90 & 183 & 3.6 & 0.85 & 196 & 2.68 & 8.27 & 184 & 10.2 & 1.40 \\
\hline Mo & 1.03 & 0.12 & 0.863 & 0.0946 & -16.26 & 1.09 & 0.178 & 5.79 & 0.926 & 0.137 & -10.14 & 0.894 & 0.122 & -13.20 \\
\hline $\mathrm{Sn}$ & 4.26 & 1.98 & 3.03 & 0.204 & -28.77 & 3.37 & 0.521 & -20.85 & 2.56 & 0.235 & -40.02 & 3.2 & 0.43 & -24.81 \\
\hline $\mathrm{Sb}$ & 1.09 & 0.17 & 1.05 & 0.23 & -3.63 & 0.806 & 0.5 & -26.10 & 1.22 & 0.27 & 12.37 & 1.05 & 0.23 & -3.63 \\
\hline Cs & 1.39 & 0.23 & 1.39 & 0.13 & -0.13 & 1.56 & 0.176 & 11.99 & 1.4 & 0.135 & 0.66 & 1.45 & 0.167 & 4.17 \\
\hline $\mathrm{Ba}$ & 919 & 18.4 & 902 & 8.7 & -1.80 & 915 & 16.6 & -0.41 & 929 & 11.9 & 1.14 & 916 & 16.9 & -0.35 \\
\hline $\mathrm{La}$ & 30.7 & 0.68 & 29.7 & 0.33 & -3.38 & 31.3 & 0.504 & 1.84 & 32.8 & 0.339 & 6.99 & 31.3 & 1.36 & 1.82 \\
\hline $\mathrm{Ce}$ & 52.7 & 1.62 & 52 & 0.447 & -1.26 & 54.7 & 0.875 & 3.75 & 55.9 & 0.726 & 6.14 & 54.2 & 1.77 & 2.88 \\
\hline $\operatorname{Pr}$ & 5.72 & 0.1 & 5.43 & 0.138 & -5.10 & 5.83 & 0.177 & 1.84 & 5.91 & 0.156 & 3.27 & 5.72 & 0.262 & 0.00 \\
\hline $\mathrm{Nd}$ & 22.4 & 0.64 & 20.8 & 0.64 & -7.17 & 22.1 & 1.01 & -1.54 & 23.4 & 0.741 & 4.61 & 22.1 & 1.35 & -1.36 \\
\hline $\mathrm{Sm}$ & 3.6 & 0.061 & 3.43 & 0.284 & -4.79 & 3.45 & 0.386 & -4.13 & 3.83 & 0.282 & 6.32 & 3.57 & 0.37 & -0.87 \\
\hline $\mathrm{Eu}$ & 1.08 & 0.057 & 1.04 & 0.103 & -4.10 & 1.05 & 0.1 & -2.52 & 1.15 & 0.114 & 6.77 & 1.08 & 0.118 & 0.05 \\
\hline $\mathrm{Gd}$ & 2.79 & 0.12 & 2.6 & 0.247 & -6.81 & 2.81 & 0.377 & 0.76 & 3.08 & 0.295 & 10.52 & 2.83 & 0.368 & 1.49 \\
\hline $\mathrm{Tb}$ & 0.37 & 0.034 & 0.321 & 0.0343 & -13.37 & 0.348 & 0.0491 & -5.89 & 0.356 & 0.038 & -3.89 & 0.341 & 0.0437 & -7.72 \\
\hline Dy & 1.91 & 0.064 & 1.72 & 0.123 & -9.82 & 1.86 & 0.137 & -2.81 & 1.96 & 0.136 & 2.49 & 1.85 & 0.163 & -3.38 \\
\hline Но & 0.35 & 0.011 & 0.318 & 0.0301 & -9.13 & 0.351 & 0.0446 & 0.15 & 0.354 & 0.0336 & 1.18 & 0.341 & 0.0401 & -2.60 \\
\hline Er & 0.94 & 0.027 & 0.864 & 0.0815 & -8.13 & 0.857 & 0.0968 & -8.86 & 0.992 & 0.0933 & 5.51 & 0.904 & 0.11 & -3.83 \\
\hline $\mathrm{Tm}$ & 0.14 & 0.013 & 0.113 & 0.0121 & -19.03 & 0.125 & 0.0127 & -10.43 & 0.126 & 0.0128 & -10.34 & 0.121 & 0.0138 & -13.27 \\
\hline $\mathrm{Yb}$ & 0.88 & 0.057 & 0.806 & 0.111 & -8.36 & 0.851 & 0.124 & -3.27 & 0.918 & 0.132 & 4.36 & 0.859 & 0.131 & -2.42 \\
\hline $\mathrm{Lu}$ & 0.13 & 0.009 & 0.117 & 0.0205 & -10.18 & 0.135 & 0.0279 & 3.91 & 0.132 & 0.0233 & 1.57 & 0.128 & 0.0254 & -1.57 \\
\hline $\mathrm{Hf}$ & 4.85 & 0.18 & 4.6 & 0.331 & -5.11 & 4.91 & 0.472 & 1.21 & 4.96 & 0.359 & 2.36 & 4.83 & 0.423 & -0.51 \\
\hline $\mathrm{Ta}$ & 0.47 & 0.085 & 0.423 & 0.039 & -9.92 & 0.464 & 0.0609 & -1.34 & 0.432 & 0.0413 & -8.19 & 0.44 & 0.0511 & -6.49 \\
\hline $\mathrm{Pb}$ & 20.7 & 2.25 & 24 & 3.88 & 15.76 & 23.4 & 3.87 & 12.82 & 25 & 4.08 & 20.92 & 24.1 & 4 & 16.50 \\
\hline Th & 7.62 & 0.37 & 7.15 & 0.248 & -6.19 & 7.66 & 0.386 & 0.50 & 8.1 & 0.225 & 6.28 & 7.63 & 0.488 & 0.20 \\
\hline $\mathrm{U}$ & 1.79 & 0.13 & 1.8 & 0.0619 & 0.82 & 1.86 & 0.0619 & 3.90 & 1.87 & 0.0567 & 4.24 & 1.84 & 0.0662 & 2.99 \\
\hline
\end{tabular}




\subsubsection{Discussions}

\subsubsection{Down-hole fractionation}

LA-ICP-MS accuracy affected by the downhole fractionation has been widely discussed (Jackson, 2008; Longerich et al., 1996), especially where the physical and/or chemical matrix of reference material does not perfectly match with analysis samples. The downhole fractionation has been explained by several mechanisms (Jackson et al., 2004), including (1) partitioning of elements preferentially into a particulate; (2) dynamic differential volatilization/condensation processes; (3) the change of particle size distribution with aspect of crater depth, thereby resulting in a different ionization efficiency in ICP (Guillong and Günther, 2002).

Here, the down-hole fractionations induced by $193 \mathrm{~nm}$ ArF laser in glass reference materials were investigated. Fig 3 shows the variations of $\mathrm{Cu} / \mathrm{Si}, \mathrm{Cu} / \mathrm{Ca}, \mathrm{Sr} / \mathrm{Si}$ and $\mathrm{Sr} / \mathrm{Ca}$ with ablation time in NIST610 and ML3B-G. The results illustrate that down-hole fractionations are dependent not only on the internal standard but also on the sample matrix (under the given laser ablation conditions in this study). The $\mathrm{Cu} / \mathrm{Si}$ was non-linearly changed with ablation time in NIS610, while nearly constant in ML3B-G. As the internal standard switched (Si to $\mathrm{Ca}$ ), the fractionation trends were changed concomitantly. The $\mathrm{Cu} / \mathrm{Ca}$ ratios were stable at initial $40 \mathrm{~s}$ and then gradually increasing in both NIST610 and ML3B-G. The Sr/Si ratios were constant in the initial $40 \mathrm{~s}$ and then decreased with ablation time, and the degrees of decreasing were slightly different for NIST610 and ML3G-B. In contrast, the Sr/Ca ratios were constant in the entire ablation time. The matrix-, elemental- and time-dependent fractionations indicate that the mechanism behind is complex. Our observed fractionations are inconsistent compared to previous studies (Gaboardi and Humayun, 2009; Hu et al., 2011; Li et al., 2015), which demonstrates that the downhole fractionation might be also influenced by the laser parameters (wavelength, spot size, energy density, and ablation time) and the geometry of ablation cell. 

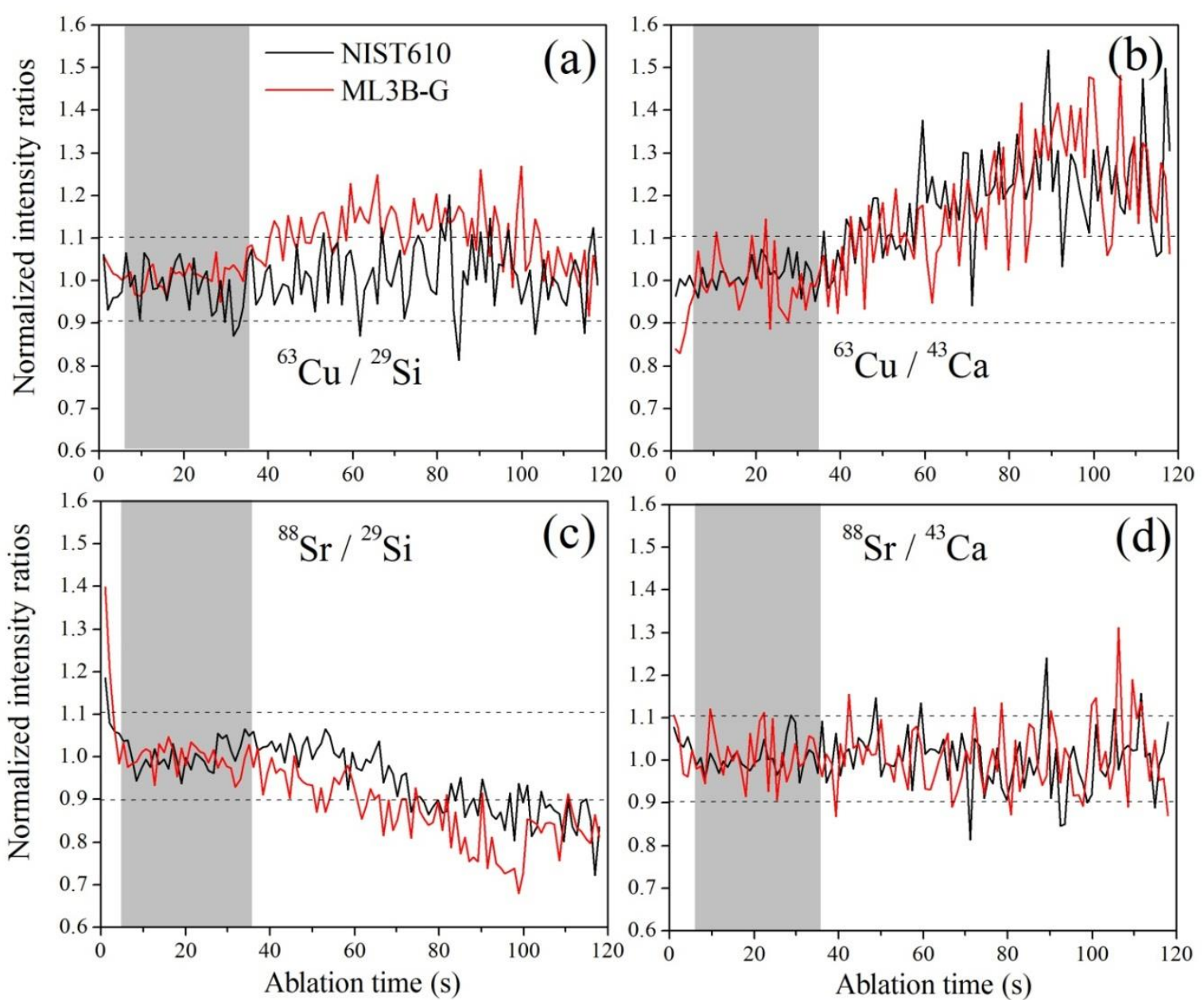

Fig. 3 Variations of $\mathrm{Cu} / \mathrm{Si}$ (a), $\mathrm{Cu} / \mathrm{Ca}(b), \mathrm{Sr} / \mathrm{Si}$ (c) and $\mathrm{Sr} / \mathrm{Ca}$ (d) with ablation time in NIST 610 and ML3B-G. All the intensity ratios are normalized to the average of the interval (5-20s) intensity ratios.

A mathematical model is widely accepted for the correction of the downhole fractionation, especially in zircon $\mathrm{Pb} / \mathrm{U}$ dating. Horn et al. (2000) observed that the $\mathrm{Pb} / \mathrm{U}$ was positive and linearly increased with the crater depth, and was inversely exponentially correlated with spot size, hence demonstrated that a linear model could efficiently correct the downhole fractionation. Paton et al. (2010) indicated that the use of more complex models such as exponential curves and smoothed cubic splines could efficiently correct complex fractionation trends. However, all the mathematical correction models are based on the assumption that the initial signal represents the sample composition; therefore these corrections may lead to a random data parallel to the initial acquisition data. The down-hole fractionations are also matrix dependent, even for glasses with different chemical 
compositions (Fig.4 a c), so that a fixed correction model could not fit for both reference material and sample. Meanwhile, it is impractical to correct the downhole fractionation for more than 40 elements (in the case of chemical bulk analysis) with any currently available software. The downhole fractionation induced by $193 \mathrm{~nm}$ wavelength laser could be reduced or even diminished with carefully optimizing laser parameters and ablation time.

Fig.3 reveals that the down-hole fractionations of NIST610 and ML3B-G in the initial 5-35s ablation period (grey zone in Fig.3) are apparently insignificant. Fig.4 shows the downhole fractionation indexes, described by Fryer et al. (1995), of selected glass reference materials in the initial 5-35s ablation period. The data illustrated that the downhole fractionation indexes of the investigated 54 elements were in the range of 0.9-1.1 (except several elements like Sn, $\mathrm{W}, \mathrm{Bi}$, that may be caused by the large standard deviation due to their relatively low concentrations). The phenomenon of highly volatile elements (like $\mathrm{Zn}$ ) may remain in the gaseous state or be partitioned into the smaller particulates during LA process (Košler et al., 2005) is not observed in the initial 5-35s ablation period. Fig.4 also reveals that the fractionation indexes are not affected by the chosen internal standards ( $\mathrm{Si}, \mathrm{Al}$, and $\mathrm{Ca}$ ). All these results demonstrated that the initial 5-35s acquisition data were free of downhole fractionation at the given laser ablation conditions in this study. It is worth emphasizing that the downhole fractionation may be heavily influenced by the laser ablation conditions so that a careful investigation of downhole fractionation for a given laser condition is a prerequisite to achieving the transient signal that is free of downhole fractionation.

IJ Fig. 4 Elemental fractionation indexes of 54 elements with $\mathrm{Al}, \mathrm{Si}$ and $\mathrm{Ca}$ as internal standards in NIST 610, NIST612, ML3B-G, StHs6/80-G, GSD-1G, BCR-2G, CGSG-1 and CGSG-4. The elemental fractionation indexes are calculated by dividing the interval 30s intensity ratios as two equal parts, and then the average of intensity ratios from the second part is divided by that of first part. A value of one indicates no down-hole fractionation. The 1s standard deviation (error bar) are derived from ten repeated analyses. 
Chapter 2.1 LA-ICP-MS Transient Signal Quantification of NIST, MPI-DING, USGS and CGSG Glass Reference Materials by Ratioing, Standardization, and Normalization (RSN)
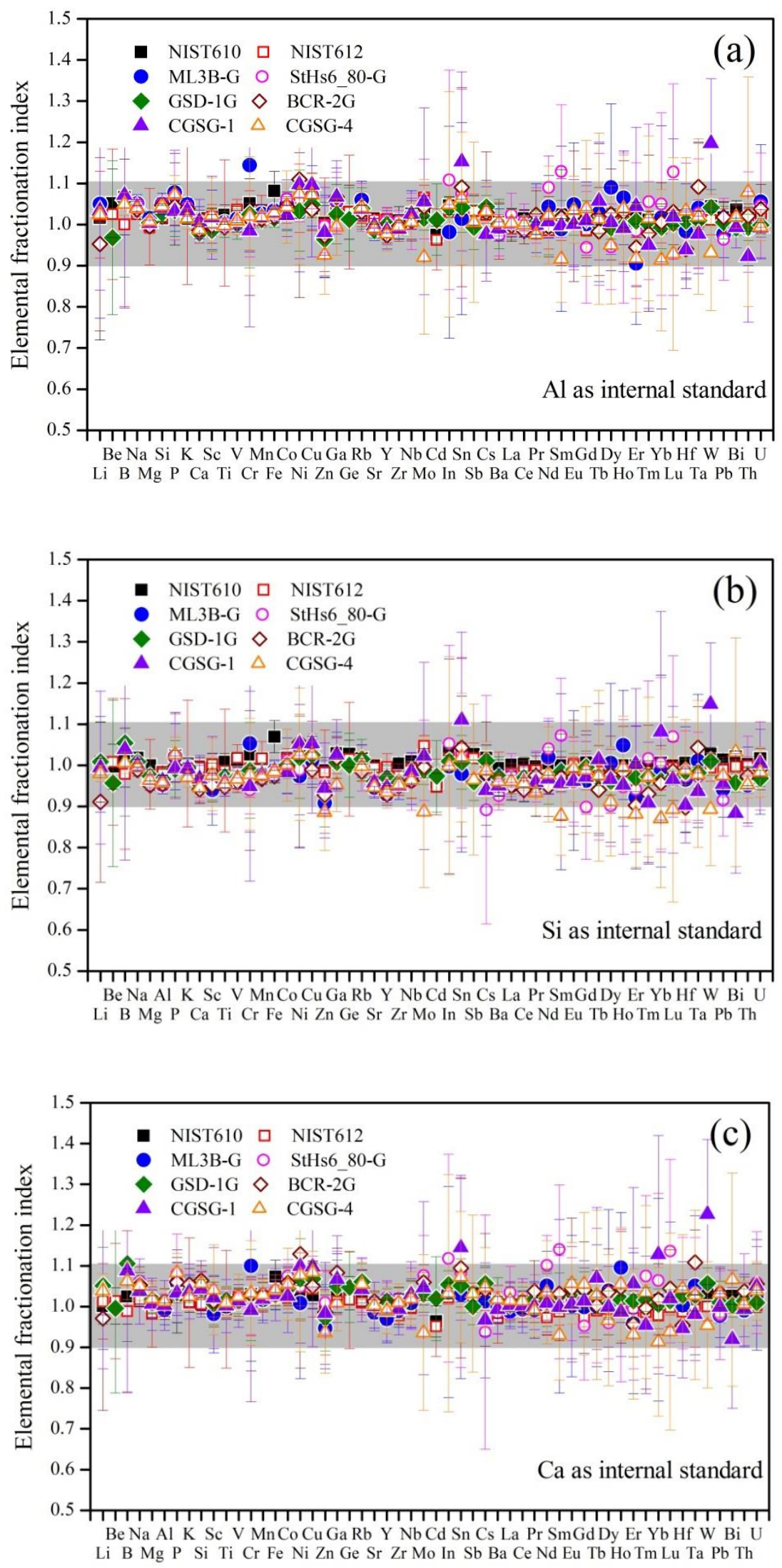


\subsubsection{Comparison with internal standardization strategy}

The internal standardization strategy has been widely used for processing LA-ICP-MS transient signal, which is normally using a natural occurring major element as the internal standard to correct the change of ablation mass for each analysis. The concentration of internal standard (usually a major element) could be obtained either through chemical stoichiometry for an ideal mineral (e.g. zircon) or via another independent technique (e.g. Electron microprobe).

The data of ML3B-G and KL-2G obtained from the internal standardization strategy with six different internal standards, including $\mathrm{Al}, \mathrm{Si}, \mathrm{Ca}, \mathrm{Cu}, \mathrm{Sr}$, and $\mathrm{Ce}$ was evaluated and compared (Fig.5 a c). The results reveal that the obtained data of ML3B-G and KL2-G were parallel to each other with respect to the internal standard. Unexpectedly, $\mathrm{Cu}$ (chalcophile element) as internal standard produced the parallel data in comparison with $\mathrm{Al}, \mathrm{Ca}, \mathrm{Sr}$ or $\mathrm{Ce}$ (lithophile element). These results are contradicted with previous knowledge that the chosen internal standard should have similar ionization efficiency compared to the targeted elements, especially for solution ICP-MS. This could be interpreted by the different ionization process in a distinct plasma environment. Laser sampling might produce relatively stable plasma (dry plasma) in comparison with liquid sampling (wet plasma). Considering that the transient signals are free of downhole fractionation (Fig.4), the parallel shifting of obtained data with respect to internal standards could be caused by the imprecise certified values of internal standard, either in external reference material or in samples.

Contrarily, RSN strategy with six internal standards produced nearly identical results for ML3B-G and KL2-G (Fig.5 b d) and was in general matched well with reference values. These data further demonstrate that the intensity ratios of two elements, even with distinct chemical character, are quite stable in the dry plasma. It is worth emphasizing that the chosen internal standard should be interference free in the aspect of mass spectroscopy, and has enough concentration to produce high intensity. For the internal standardization strategy, the given value of internal standard heavily affects the analytical accuracy. However, for the 
Chapter 2.1 LA-ICP-MS Transient Signal Quantification of NIST, MPI-DING, USGS and CGSG Glass Reference Materials by Ratioing, Standardization, and Normalization (RSN)

RSN strategy, the analytical accuracy is independent of the chosen internal standard, while the uncertainties of bulk normalization as $100 \% \mathrm{~m} / \mathrm{m}$ that are derived from the assumption of multivalent element state, missed components and accuracy of single point calibration are partly influencing the data accuracy.
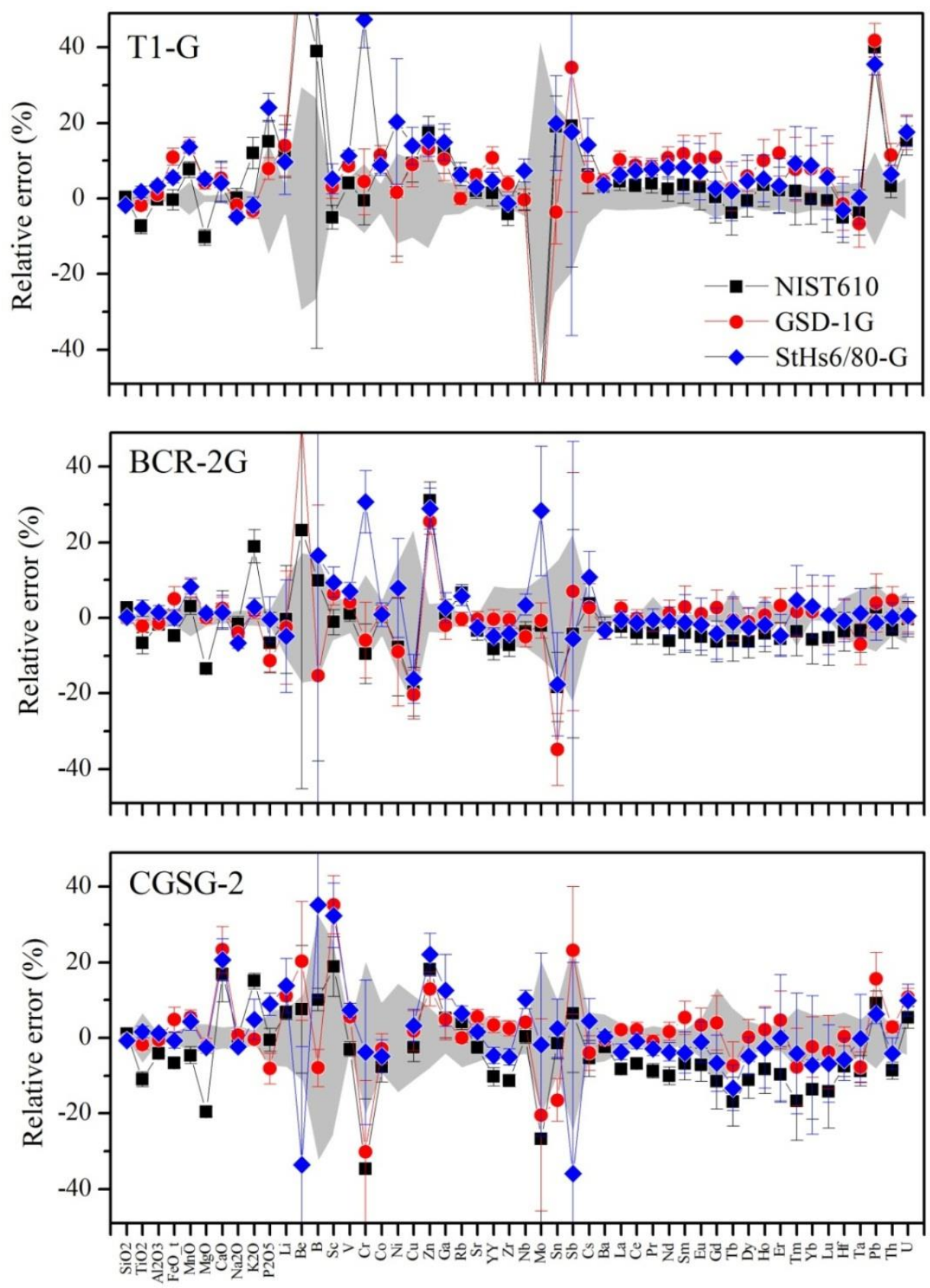

Fig. 6 The concentration data of KL-2G, BCR-2G and CGSG-4 obtained from RSN strategy with Si as internal standard and NIST610, GSD-1G and StHs6/80-G as external reference materials. The grey zone represents the uncertainty of certified reference values. The LA-ICP-MS means and standard deviations of KL2-G, BCR-2G and CGSG-2 are derived from 78, 73 and 29 repeated analyses. 


\subsubsection{Standardization with different external reference materials}

The potential matrix effect and the quality of reference values in external reference material are strongly controlling the accuracy of LA-ICP-MS (Gaboardi and Humayun, 2009; Li et al., 2015; Luo et al., 2007). Here we plotted the data of T1-G, BCR-2G, and CGSG-2 that are obtained from RSN strategy with NIST610, StHs6/80-G, and GSD-1G as external reference materials (Fig.6). The results reveal that the accuracy obtained with NIST610, StHs6/80-G, and GSD-1G was a slight difference, but within analytical uncertainty (except some major elements calibrated with NIST610). This indicates that the matrix effects between NIST and MPI-DING, USGS glasses are insignificant. Liu et al. (2008) observed a serious matrix effect existing between NIST and geological glasses. That may be caused by the different ablation conditions, which can heavily affect the downhole fractionation, thus induce the matrix effects (Li et al., 2015).

The accurate measurement of the major element is the key point for RSN strategy, and it directly introduces uncertainty to the normalization part. Relative lower or higher (not larger than $20 \%$ observed in this study) data of $\mathrm{TiO}_{2}, \mathrm{FeO}_{-} \mathrm{t}, \mathrm{MgO}, \mathrm{MnO}$ and $\mathrm{K}_{2} \mathrm{O}$ in T1-G, BCR-2G and CGSG-2 were observed when NIST610 works as the calibration standard. This phenomenon is probably related to the large uncertainty in single point calibration due to their relatively low concentrations in NIST 610 (for example, $589 \mu \mathrm{g} / \mathrm{g}$ of FeO_t in NIST610 to calibrate $12.4 \mathrm{wt} \%$ in BCR-2G). However, the sum of these five major components in T1-G, BCR-2G, and CGSG-2 (also in other geological glasses) is smaller than 20\%, thus the uncertainty propagated to the normalization part is maximum $4 \%$, which is, in general, acceptable when considering the overall uncertainty for determining trace elements (around $10 \%$ ). We could conclude that NIST610 as external reference material may not produce good agreement data for some major elements, but data for trace elements are generally acceptable.

Fig. 5 Element concentrations of ML3B-G and KL2-G obtained from internal standardization strategy and RSN strategy with six internal standards that includes $\mathrm{Al}, \mathrm{Si}, \mathrm{Ca}, \mathrm{Cu}, \mathrm{Sr}$ and $\mathrm{Ce}$. Data are standardized with NIST610 
Chapter 2.1 LA-ICP-MS Transient Signal Quantification of NIST, MPI-DING, USGS and CGSG Glass Reference Materials by Ratioing, Standardization, and Normalization (RSN)

as external reference material. The 1s standard deviation (error bar) are derived from ten repeated analyses (data from sequence 7). Grey zone represents the uncertainty of reference values.
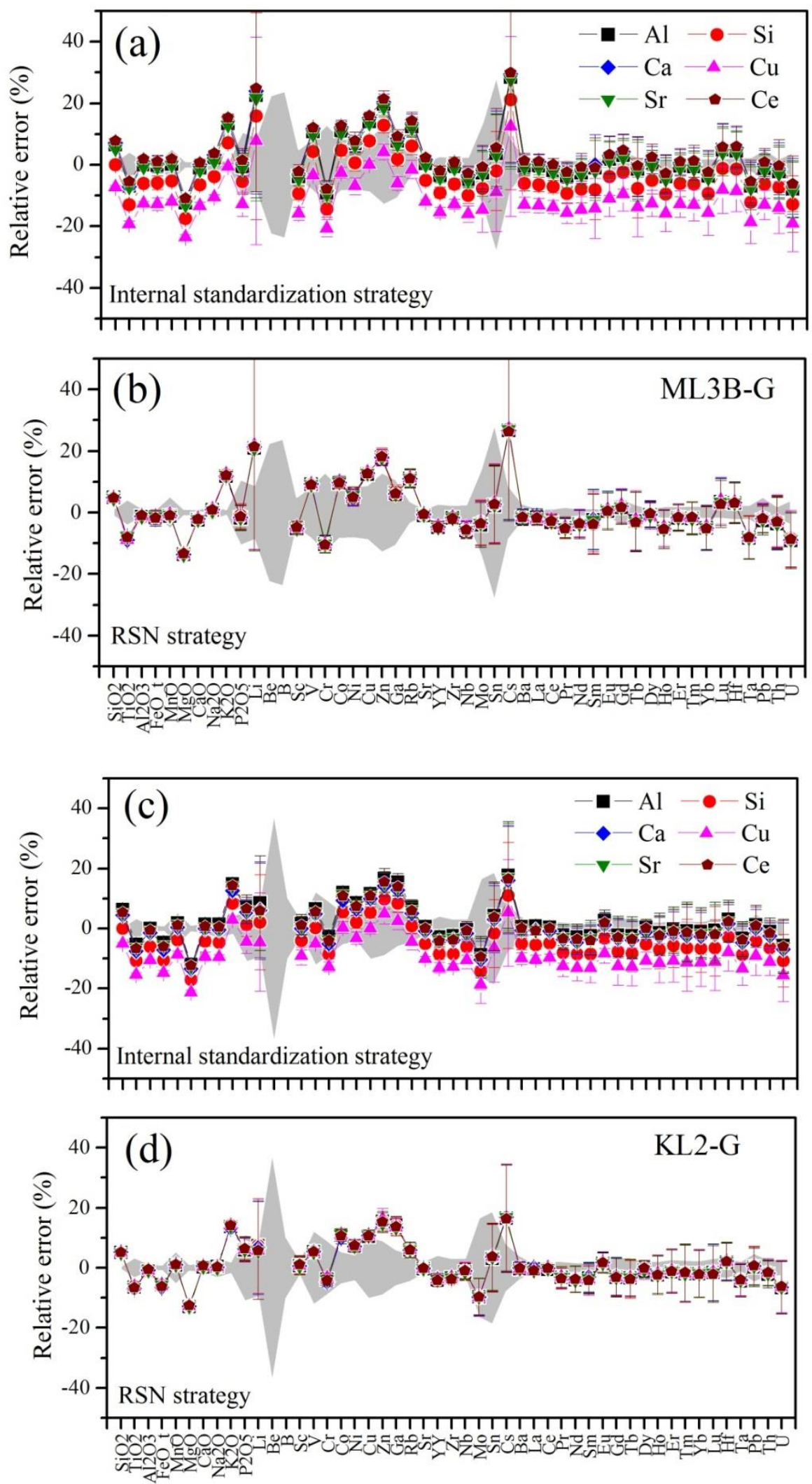
The concentrations of $\mathrm{Mo}, \mathrm{Pb}$, Th in $\mathrm{T} 1-\mathrm{G}$ and $\mathrm{Zn}$ in BCR-2G standardized with NIST610, GSD-1G and StHs6/80-G were identically lower or higher, which reveals that the certified values of those elements in $\mathrm{T} 1-\mathrm{G}$ and $\mathrm{BCR}-2 \mathrm{G}$ probably are imprecise. The concentrations of $\mathrm{Cr}$ in T1-G and BCR-2G calibrated with StHs6/80-G were out a range of value uncertainty, while the data calibrated with NIST610 and GSD-1G were generally matched with certified values, which demonstrates that the $\mathrm{Cr}$ in StHs6/80-G may have an overestimated value. The data in CGSG-2 calibrated with three external reference materials were generally matched worse with certified value compared to that for T1-G and BCR-2G. This is probably caused by the large uncertainty of certified value in CGSG-2. In summary, the crucial limitation for LA-ICP-MS accuracy is the uncertainty of external reference materials. Moreover, the data demonstrate that the matrix effects between NIST610 and geological glasses are insignificant at the given laser ablation conditions, or at least smaller than the analytical precision.

\subsubsection{Effect of spot size to RSN strategy}

As the intensive demands of chemical analysis at high spatial resolution, especial for the application of mineral elemental mapping (Raimondo et al., 2017; Ubide et al., 2015), smaller laser spot size is required. Smaller spot size ablation could provide more detailed chemical signatures (Li et al., 2015; Pearce et al., 2011; Wu et al., 2016). Here we carried out an experiment to evaluate the applicability of RSN strategy with respect to laser spot size. The results illustrated that most data obtained from RSN strategy with the spot size down to 15 $\mu \mathrm{m}$ in $\mathrm{KL}-2 \mathrm{G}$ and BCR-2G were generally in agreement with certified values within $15 \%$, expect $\mathrm{Li}, \mathrm{Cr}$ and $\mathrm{Ni}$ in BCR-2G (Fig.7), which demonstrates that $15 \mu \mathrm{m}$ is the smallest spot size where the applicability of RSN strategy is acceptable. The increase of relative standard deviation (RSD \%) with a decrease of spot size is related to counting statistics uncertainty. With the technical developments including modified sample/skimmer cones (Hu et al., 2012), improved vacuum system and strengthened plasma (small amount of inert gas $\left(\mathrm{N}_{2}\right.$ and $\left.\mathrm{H}_{2}\right)$ into sample gas (Hu et al., 2008), the higher spatial resolution at sub-ten microns could be achieved in near future. 
Chapter 2.1 LA-ICP-MS Transient Signal Quantification of NIST, MPI-DING, USGS and CGSG Glass Reference Materials by Ratioing, Standardization, and Normalization (RSN)
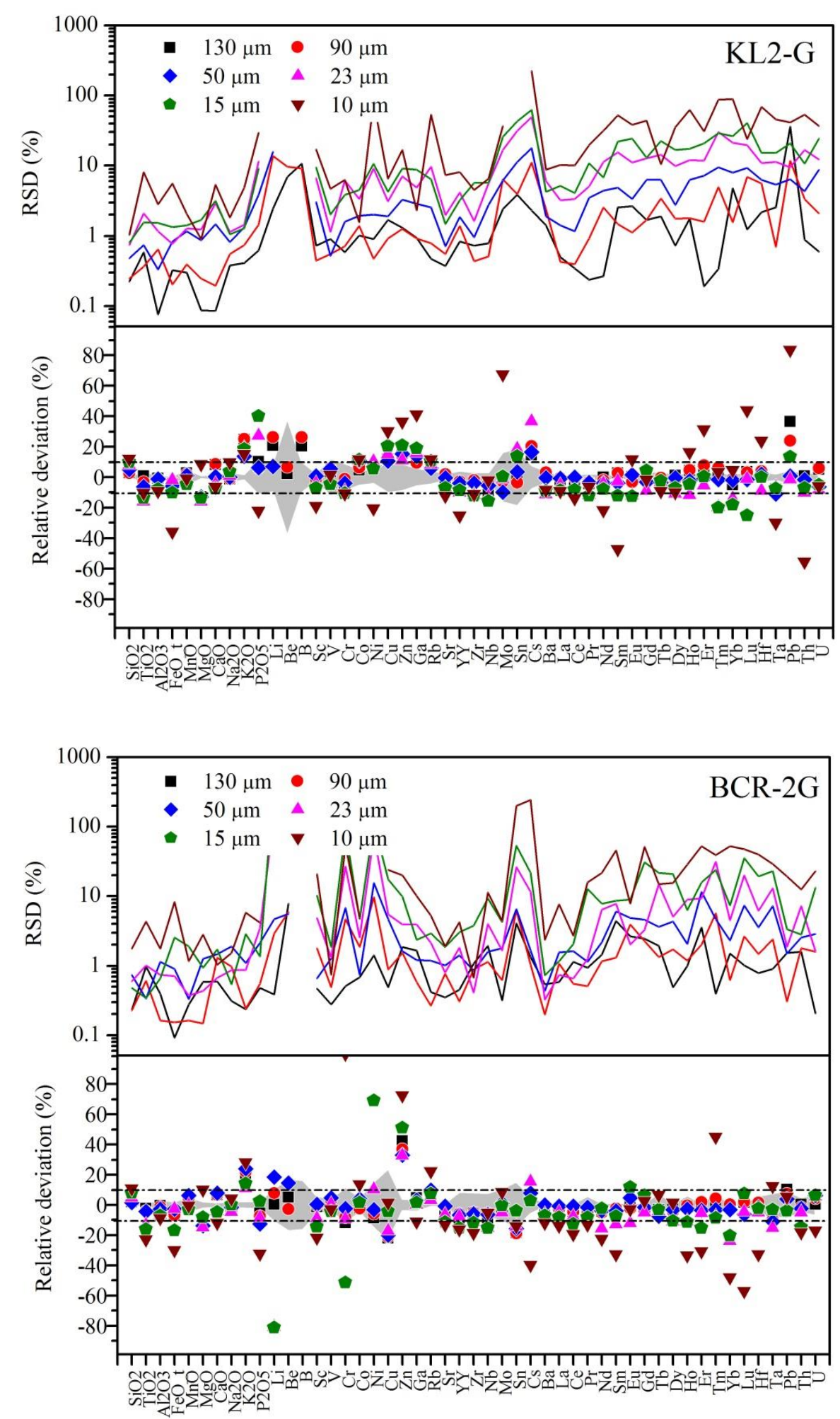

Fig. 7 Element concentrations of KL-2G and BCR-2G obtained from RSN strategy at different laser spot size $(10,15,23,50,90$ and $130 \mu \mathrm{m})$. Data were calculated with Si as internal standard and NIST610 as external reference material. The LA-ICP-MS means and standard deviations are derived from three repeated analyses. 
Chapter 2.1 LA-ICP-MS Transient Signal Quantification of NIST, MPI-DING, USGS and CGSG Glass Reference Materials by Ratioing, Standardization, and Normalization (RSN)

\subsubsection{Analytical precision}

The analytical precision that includes short-term precision and long-term precision is a crucial parameter for the instrument conditions as well as data reduction strategy.

\subsubsection{Short-term precision}

Fig.8 illustrates the correlation between concentration and RSD (\%) of major, lithophile and siderophile/chalcophile elements in MPI-DING and USGS glasses. The elemental concentrations were obtained from RSN strategy with Si as internal standard and NIST610 as external reference material. The short-term precision given as 1 s relative standard deviation ( $\mathrm{n}=10)$ was less than $10 \%$ for most elements with concentrations larger than $0.1 \mu \mathrm{g} / \mathrm{g}$ (Fig.8), which is approvingly acceptable for the application in geochemistry. There was a significant negative linear correlation between concentration and RSD in logarithmic scale (Fig.8). The trend of decrease of RSD with increasing concentration was following the dashed line (Poisson counting uncertainty) in Fig.9, which demonstrates that the analytical precision is derived from the original analysis, thus the potential chemical heterogeneity could be excluded (Gao et al., 2002; He et al., 2016; Z Hu et al., 2009; Wu et al., 2016). The results indicated that the RSD of siderophile/chalcophile was slightly higher than that of Lithophile elements. This is probably due to the difference of ICP-MS sensitivity for each element. The RSD of the major elements did not decrease with the increase of concentration when RSD reached to around $0.2 \%$, which infers that $0.2 \%$ may be the instrument stability (the highest precision that the instrument could reach).

$\sqrt{ }$ Fig. 8 Concentrations versus RSD (\%) of major, lithophile and siderophile/chalcophile elements in MPI-DING and USGS glasses. Concentrations are obtained from RSN strategy. The RSD are derived from ten repeated analysis (spot size is $50 \mu \mathrm{m}$ ). The data are from analysis sequence 7 . The dashed line indicates the Poisson counting uncertainty which is calculated based on the Ce sensitivity. The solid line represents the lowest precision the instrument could reach.

Major elements include Al, Mg, Ca, $\mathrm{Na}$ and K. Lithophile elements include $\mathrm{Sc}, \mathrm{Rb}, \mathrm{Sr}, \mathrm{Y}, \mathrm{Zr}, \mathrm{Nb}, \mathrm{Cs}, \mathrm{Ba}, \mathrm{La}$, Ce, Pr, Nd, Sm, Eu, Gd, Tb, Dy, Ho, Er, Tm, Yb, Lu, Ta, Th and U. Siderophile/chalcophile elements include V, Cr, Co, Ni, Cu, Zn, Ga, Mo, Sn, Sb, W, Tl, Pb and Bi. 


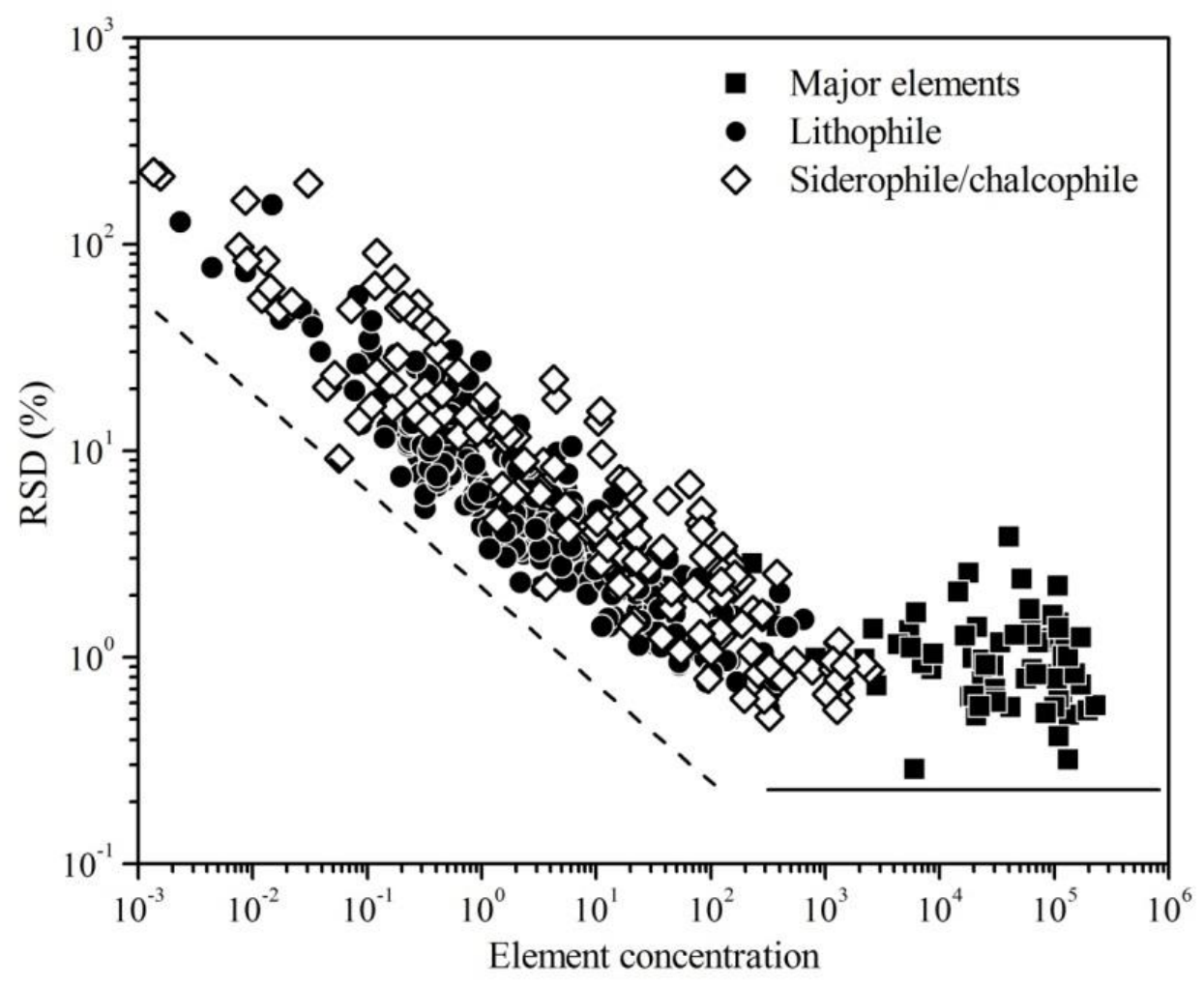

\subsubsection{Long-term precision}

The long-term precision has been established via repeated analysis of StHs6/80-G with Si as internal standard and NIST610 as external reference material. StHs6/80-G was treated as unknown for the quality control purposes in every analysis sequence in our laboratory over past 3 years. The results illustrated that all the data fell in the range of $2 \mathrm{~s}$ standard deviation (Fig.9 a), which demonstrates that the instrument conditions, as well as RSN strategy, is quite stable. Fig.9 b reveals the histogram of 359 analyses. The data indicated that 359 analyses were following a Gauss distribution. However, the calculated mean of Ce concentration in StHs6/80 was slightly shifted from the certified value. This is probably caused by the uncertainty of Ce reference value in StHs6/80-G or NIST610. 

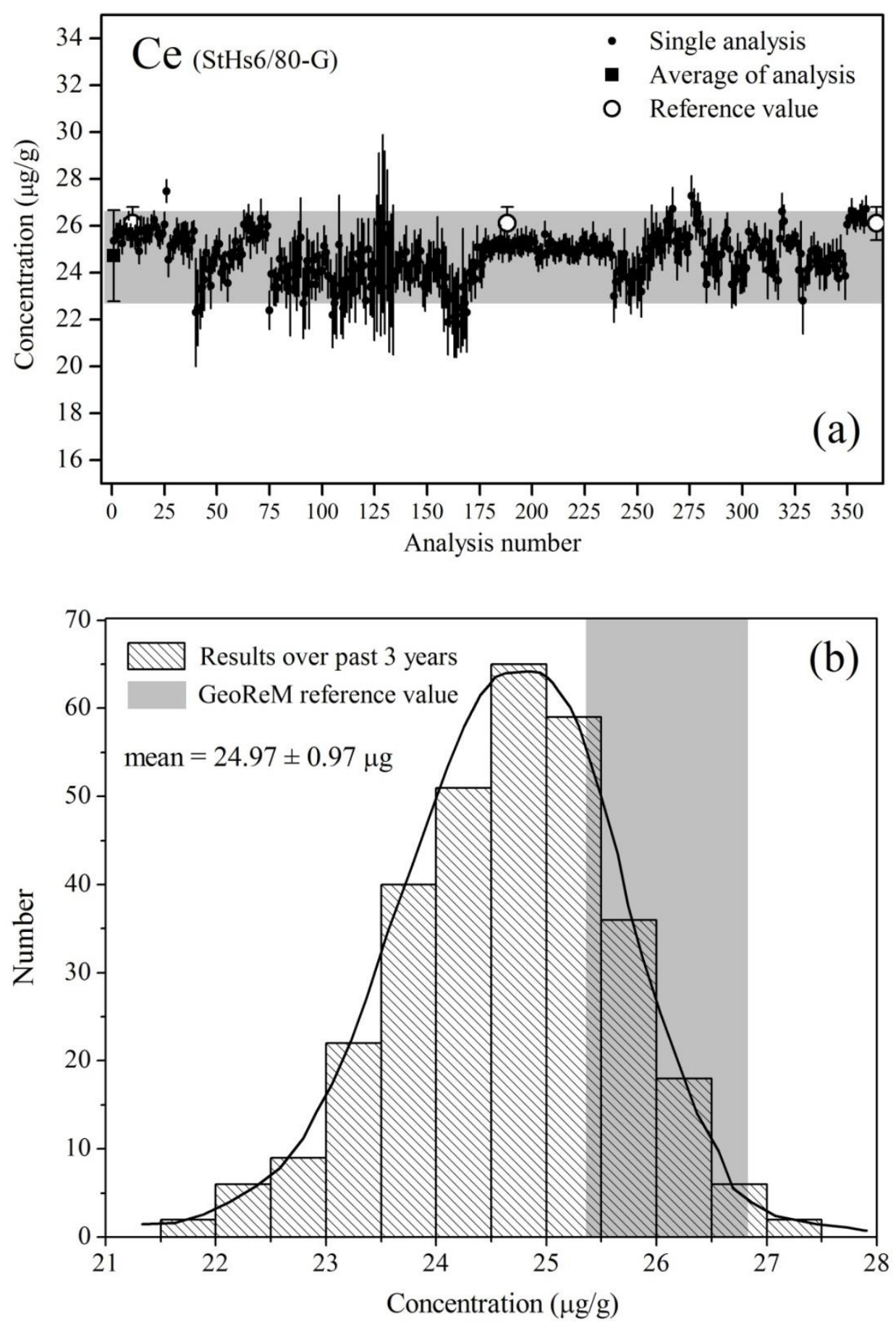

Fig. 9 (a) Variation of Ce concentrations in StHs 6/80-G with 359 repeated analyses over three years. The grey zone is two times of standard deviation derived from 359 analyses. (b) Frequency distribution histogram of Ce concentrations in StHs6/80-G. "mean $\pm 1 \mathrm{~s}$ " are calculated based on 359 repeated analyses. The Ce concentrations in StHs6/80-G are obtained with RSN strategy where Si as internal standards and NIST610 as external reference material. The laser spot size varies from 15 to $130 \mu \mathrm{m}$, and the laser energy density varies in a range of 3.0-6.0 J/cm² 


\subsection{Evaluation of reference values}

The preferred values in the data set (Table 3) illustrated that most major and lithophile elements (REEs, Th, U et al.) are matched well with reference values (within $3 \%$ ), and transition metal are generally matched within $15 \%$. Those transition metal are potential heterogeneity distributed compared to lithophile element (personal communication with Klaus Peter Jochum). However, for some elements (like Sc in ATHO-G), three external reference materials gave identical more than $40 \%$ larger values compared to the certified value (Fig.10), which demonstrates the certified value of Sc in ATHO-G is probably imprecise. Similar case for Co, Mo in ATHO-G, Cs in GOR128-G, Mo, Pb, U in T1-G, Cr, $\mathrm{Ni}$ in StHs6/80-G, U in BIR-1G, Zn in BHVO-2G and Sc, Cr in CGSG-2, and the prefer values reported in this study might be the better-estimated values for these elements.

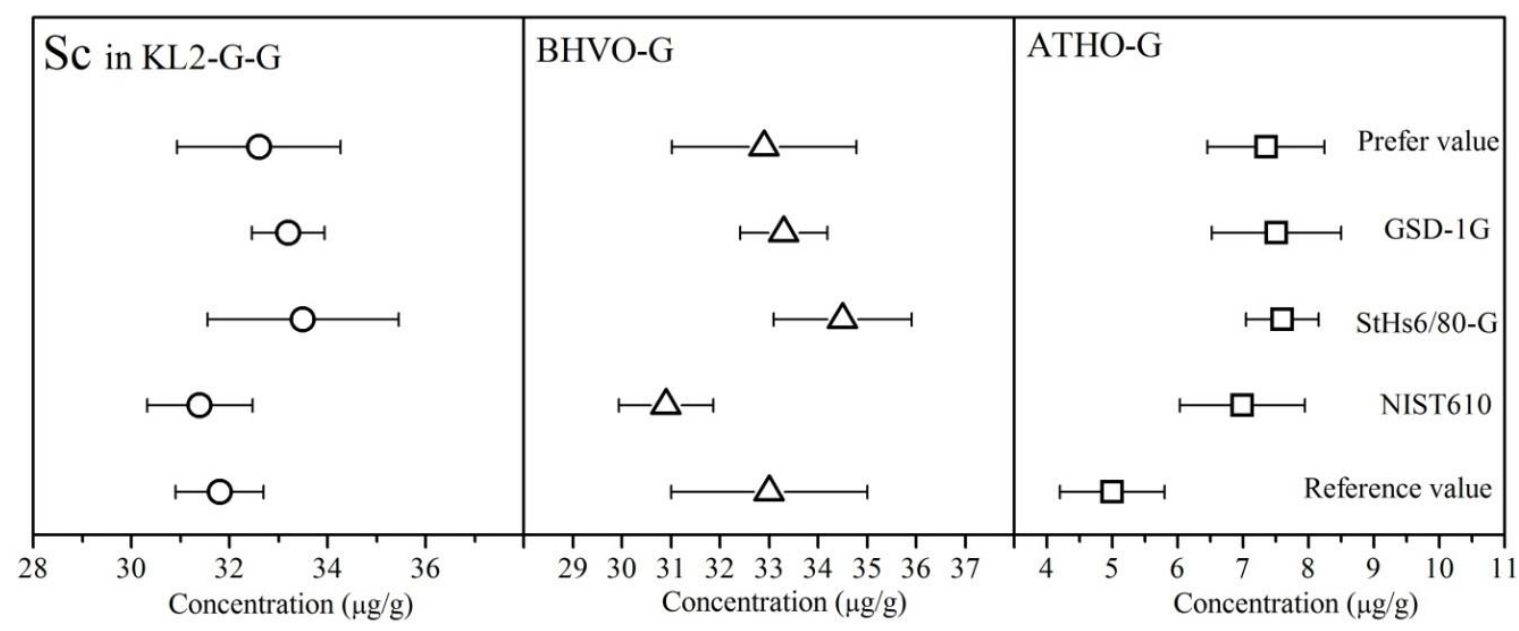

Fig. 10 Comparison of reference value, prefer value and concentrations obtained with NIST610, StHs6/80-G and GSD-1G as external reference materials of Sc in KL2-G, BHVO-G and ATHO-G. 


\subsubsection{Conclusions}

The RSN strategy consisting of Ratioing, Standardization, and Normalization was proposed to process the LA-ICP-MS transient signal of NIST610, MPI-DING, USGS, and CGSG glass reference materials. Its capability and applicability were evaluated from aspects of internal standards, external reference materials and short-, long-term precision. The results illustrated the down-hole fractionations in initial 5-35s ablation period were insignificant and independent of the chosen internal standards under the given laser ablation conditions. The accuracy of internal standardization strategy was dependent on the internal standard chosen (the given reference value), which might be caused by the imprecise certified value of the internal standard. On the contrary, the RSN strategy was not influenced by the internal standards. The external reference materials (NIST610, GSD-1G, and StHs6/80-G) produced similar accuracy for most elements, which demonstrates that the matrix effect between NIST610 and geological glasses is insignificant. The data of several elements obtained from NIST610, StHs6/80-G, and GSD-1G were matched well with each other but shifted from the certified values, which reveals the potential imprecise of certified values. The prefer values reported in this study are the better-estimated values for these elements. The agreement of data obtained from RSN strategy and reference values was better than $15 \%$ for most elements in KL2-G and BCR-2G as the spot size down to $15 \square \mathrm{m}$. The short-term precision given as 1s relative standard deviation $(\mathrm{n}=10)$ was less than $10 \%$ for most elements with concentrations larger than $0.1 \square \mathrm{g} / \mathrm{g}$. The long-term precision (359 repetition analyses over 3 years) illustrated that either instrument conditions or the capability of RSN strategy were approvingly acceptable. The simplicity and applicability of RNS strategy in comparison with internal standardization strategy make it more suitable for quantitative data reduction for silicate glasses. 


\section{Acknowledgements}

A greatly thankful is given to Prof. Dr. Xiuchun Zhan for providing of CGSG reference materials and the reference values. We also thank Klaus Peter Jochum for supplying MPI-DING reference glasses. Financial support from Göttingen University (LA-ICP-MS laboratory) and China Scholarship Committee (Nr.: 201306410007) are greatly acknowledged.

\section{References}

Cruz-Uribe AM, Mertz-Kraus R, Zack T, Feineman MD, Woods G, Jacob DE (2016) A New LA-ICP-MS Method for Ti in Quartz: Implications and Application to High Pressure Rutile - Quartz Veins from the Czech Erzgebirge. Geostand. Geoanal. Res. 41(1): 29-40

Czas J, Jochum KP, Stoll B, Weis U, Yang QC, Jacob DE, Andreae MO (2012) Investigation of matrix effects in $193 \mathrm{~nm}$ laser ablation-inductively coupled plasma-mass spectrometry analysis using reference glasses of different transparencies. Spectroc. Acta Pt. B-Atom. Spectr. 78: 20-28

Eggins SM, Kinsley LPJ, Shelley JMG (1998) Deposition and element fractionation processes during atmospheric pressure laser sampling for analysis by ICP-MS. Appl. Surf. Sci. 127-129: 278-286

Fietzke J, Frische M (2016) Experimental evaluation of elemental behavior during LA-ICP-MS: influences of plasma conditions and limits of plasma robustness. J. Anal. At. Spectrom. 31(1): 234-244 
Chapter 2.1 LA-ICP-MS Transient Signal Quantification of NIST, MPI-DING, USGS and CGSG Glass Reference Materials by Ratioing, Standardization, and Normalization (RSN)

Fryer BJ, Jackson SE, Longerich HP (1995) The design, operation and role of the laser-ablation microprobe coupled with an inductively coupled plasma-mass spectrometer (LAM-ICP-MS) in the earth sciences. Canadian Mineralogist. 33: 303-312

Günther D, Heinrich CA (1999) Comparison of the ablation behaviour of $266 \mathrm{~nm} \mathrm{Nd:} \mathrm{YAG}$ and $193 \mathrm{~nm}$ ArF excimer lasers for LA-ICP-MS analysis. J. Anal. At. Spectrom. 14(9): $1369-1374$

Gaboardi M, Humayun M (2009) Elemental fractionation during LA-ICP-MS analysis of silicate glasses: implications for matrix-independent standardization. J. Anal. At. Spectrom. 24(9): 1188-1197

Gagnon JE, Fryer BJ, Samson IM, Williams-Jones AE (2008) Quantitative analysis of silicate certified reference materials by LA-ICPMS with and without an internal standard. J. Anal. At. Spectrom. 23(11): 1529-1537

Gao S, Liu XM, Yuan HL, Hattendorf B, Günther D, Chen L, Hu SH (2002) Determination of Forty Two Major and Trace Elements in USGS and NIST SRM Glasses by Laser Ablation-Inductively Coupled Plasma-Mass Spectrometry. Geostandards Newsletter. 26(2): $181-196$

Garbe-Schönberg D, Müller S (2014) Nano-particulate pressed powder tablets for LA-ICP-MS. J. Anal. At. Spectrom. 29 (6): 990-1000

Gray AL (1985) Solid sample introduction by laser ablation for inductively coupled plasma source mass spectrometry. Analyst. 110(5): 551-556 
Guillong M, Günther D (2002) Effect of particle size distribution on ICP-induced elemental fractionation in laser ablation-inductively coupled plasma-mass spectrometry. J. Anal. At. Spectrom. 17(8): 831-837

Guillong M, Hametner K, Reusser E, Wilson SA, Günther D (2005) Preliminary Characterisation of New Glass Reference Materials (GSA-1G, GSC-1G, GSD-1G and GSE-1G) by Laser Ablation-Inductively Coupled Plasma-Mass Spectrometry Using 193 nm, $213 \mathrm{~nm}$ and $266 \mathrm{~nm}$ Wavelengths. Geostand. Geoanal. Res. 29(3): 315-331

Guillong M, Horn I, Günther D (2003) A comparison of $266 \mathrm{~nm}, 213 \mathrm{~nm}$ and $193 \mathrm{~nm}$ produced from a single solid state Nd:YAG laser for laser ablation ICP-MS. J. Anal. At. Spectrom. 18(10): 1224-1230

Halicz L, Günther D (2004) Quantitative analysis of silicates using LA-ICP-MS with liquid calibration. J. Anal. At. Spectrom. 19(12): 1539-1545

He Z, Huang F, Yu H, Xiao Y, Wang F, Li Q, Xia Y, Zhang X (2016) A Flux-Free Fusion Technique for Rapid Determination of Major and Trace Elements in Silicate Rocks by LA-ICP-MS. Geostand. Geoanal. Res. 40(1): 5-21

Horn I, Rudnick RL, McDonough WF (2000) Precise elemental and isotope ratio determination by simultaneous solution nebulization and laser ablation-ICP-MS: application to U-Pb geochronology. Chem. Geol. 164(3-4): 281-301

Horn I, von Blanckenburg F (2007) Investigation on elemental and isotopic fractionation during $196 \mathrm{~nm}$ femtosecond laser ablation multiple collector inductively coupled plasma mass spectrometry. Spectrochimica Acta Part B: Atomic Spectroscopy. 62(4): 410-422 
Hu Z, Gao S, Liu Y, Xu J, Hu S, Chen H (2008) Niobium and tantalum concentrations in NIST SRM 610 revisited. Geostand. Geoanal. Res. 32(3): 347-360

Hu Z, Liu Y, Gao S, Liu W, Zhang W, Tong X, Lin L, Zong K, Li M, Chen H (2012) Improved in situ $\mathrm{Hf}$ isotope ratio analysis of zircon using newly designed $\mathrm{X}$ skimmer cone and jet sample cone in combination with the addition of nitrogen by laser ablation multiple collector ICP-MS. J. Anal. At. Spectrom. 27(9): 1391-1399

Hu Z, Liu Y, Li M, Gao S, Zhao L (2009) Results for Rarely Determined Elements in MPI DING, USGS and NIST SRM Glasses Using Laser Ablation ICP - MS. Geostand. Geoanal. Res. 33(3): 319-335

Hu ZC, Gao S, Liu YS, Hu SH, Chen HH, Yuan HL (2008) Signal enhancement in laser ablation ICP-MS by addition of nitrogen in the central channel gas. J. Anal. At. Spectrom. 23(8): 1093-1101

Hu ZC, Liu YS, Chen L, Zhou LA, Li M, Zong KQ, Zhu LY, Gao S (2011) Contrasting matrix induced elemental fractionation in NIST SRM and rock glasses during laser ablation ICP-MS analysis at high spatial resolution. J. Anal. At. Spectrom. 26(2): 425-430

J. G. Mank A, R. D. Mason P (1999) A critical assessment of laser ablation ICP-MS as an analytical tool for depth analysis in silica-based glass samples. J. Anal. At. Spectrom. 14(8): $1143-1153$

Jackson SE (2008) Calibration strategies for elemental analysis by LA-ICP-MS. Laser Ablation ICP-MS in the Earth Sciences: Current Practices and Outstanding Issues (P. Sylvester, ed.). Mineralogical Association of Canada. Short Course Series. 40: 169-188 
Jackson SE, Pearson NJ, Griffin WL, Belousova EA (2004) The application of laser ablation-inductively coupled plasma-mass spectrometry to in situ $\mathrm{U}-\mathrm{Pb}$ zircon geochronology. Chem. Geol. 211(1): 47-69

Jochum KP, Enzweiler J: Reference Materials in Geochemical and Environmental Research: Treatise on Geochemistry (Second Edition); Elsevier, Oxford 2014, 43-70

Jochum KP, Stoll B, Herwig K, Willbold M, Hofmann AW, Amini M, Aarburg S, Abouchami W, Hellebrand E, Mocek B, Raczek I, Stracke A, Alard O, Bouman C, Becker S, Dücking M, Brätz H, Klemd R, de Bruin D, Canil D, Cornell D, de Hoog C-J, Dalpé C, Danyushevsky L, Eisenhauer A, Gao Y, Snow JE, Groschopf N, Günther D, Latkoczy C, Guillong M, Hauri EH, Höfer HE, Lahaye Y, Horz K, Jacob DE, Kasemann SA, Kent AJR, Ludwig T, Zack T, Mason PRD, Meixner A, Rosner M, Misawa K, Nash BP, Pfänder J, Premo WR, Sun WD, Tiepolo M, Vannucci R, Vennemann T, Wayne D, Woodhead JD (2006) MPI-DING reference glasses for in situ microanalysis: New reference values for element concentrations and isotope ratios. Geochemistry, Geophysics, Geosystems. 7(2): $1-44$

Jochum KP, Stoll B, Weis U, Jacob DE, Mertz-Kraus R, Andreae MO (2014) Non-Matrix-Matched Calibration for the Multi-Element Analysis of Geological and Environmental Samples Using $200 \mathrm{~nm}$ Femtosecond LA-ICP-MS: A Comparison with Nanosecond Lasers. Geostand. Geoanal. Res. 38(3): 265-292

Jochum KP, Weis U, Stoll B, Kuzmin D, Yang QC, Raczek I, Jacob DE, Stracke A, Birbaum K, Frick DA, Günther D, Enzweiler J (2011) Determination of Reference Values for NIST SRM 610-617 Glasses Following ISO Guidelines. Geostand. Geoanal. Res. 35(4): 397-429 
Koch J, Feldmann I, Jakubowski N, Niemax K (2002) Elemental composition of laser ablation aerosol particles deposited in the transport tube to an ICP. Spectrochimica Acta Part B: Atomic Spectroscopy. 57(5): 975-985

Koch J, Wälle M, Dietiker R, Günther D (2008) Analysis of Laser-Produced Aerosols by Inductively Coupled Plasma Mass Spectrometry: Transport Phenomena and Elemental Fractionation. Analytical Chemistry. 80(4): 915-921

Košler J, Wiedenbeck M, Wirth R, Hovorka J, Sylvester P, Míková J (2005) Chemical and phase composition of particles produced by laser ablation of silicate glass and zircon-implications for elemental fractionation during ICP-MS analysis. J. Anal. At. Spectrom. 20(5): 402-409

Kovacs R, Günther D (2008) Influence of transport tube materials on signal response and drift in laser ablation-inductively coupled plasma-mass spectrometry. J. Anal. At. Spectrom. 23(9): 1247-1252

Kroslakova I, Günther D (2007) Elemental fractionation in laser ablation-inductively coupled plasma-mass spectrometry: evidence for mass load induced matrix effects in the ICP during ablation of a silicate glass. J. Anal. At. Spectrom. 22(1): 51-62

Li Z, Hu Z, Liu Y, Gao S, Li M, Zong K, Chen H, Hu S (2015) Accurate determination of elements in silicate glass by nanosecond and femtosecond laser ablation ICP-MS at high spatial resolution. Chem. Geol. 400: 11-23

Liu YS, Hu ZC, Gao S, Gunther D, Xu J, Gao CG, Chen HH (2008) In situ analysis of major and trace elements of anhydrous minerals by LA-ICP-MS without applying an internal standard. Chem. Geol. 257(1-2): 34-43 
Liu YS, Hu ZC, Li M, Gao S (2013) Applications of LA-ICP-MS in the elemental analyses of geological samples. Chinese Science Bulletin. 58(32): 3863-3878

Longerich H, Günther D, Jackson S (1996) Elemental fractionation in laser ablation inductively coupled plasma mass spectrometry. Fresenius' journal of analytical chemistry. 355(5): $538-542$

Longerich HP, Jackson SE, Günther D (1996) Inter-laboratory note. Laser ablation inductively coupled plasma mass spectrometric transient signal data acquisition and analyte concentration calculation. J. Anal. At. Spectrom. 11(9): 899-904

Luo Y, Gao S, Longerich HP, Günther D, Wunderli S, Yuan HL, Liu XM (2007) The uncertainty budget of the multi-element analysis of glasses using LA-ICP-MS. J. Anal. At. Spectrom. 22(2): 122-130

Müller W, Shelley M, Miller P, Broude S (2009) Initial performance metrics of a new custom-designed ArF excimer LA-ICPMS system coupled to a two-volume laser-ablation cell. J. Anal. At. Spectrom. 24(2): 209-214

Paton C, Hellstrom J, Paul B, Woodhead J, Hergt J (2011) Iolite: Freeware for the visualisation and processing of mass spectrometric data. J. Anal. At. Spectrom. 26(12): $2508-2518$

Paton C, Woodhead JD, Hellstrom JC, Hergt JM, Greig A, Maas R (2010) Improved laser ablation $\mathrm{U}-\mathrm{Pb}$ zircon geochronology through robust downhole fractionation correction. Geochemistry, Geophysics, Geosystems. 11(3): 1-36 
Pearce NJ, Perkins WT, Westgate JA, Wade SC (2011) Trace-element microanalysis by LA-ICP-MS: the quest for comprehensive chemical characterisation of single, sub-10 $\mu \mathrm{m}$ volcanic glass shards. Quaternary International. 246(1): 57-81

Pearce NJG, Perkins WT, Westgate JA, Gorton MP, Jackson SE, Neal CR, Chenery SP (1997) A Compilation of New and Published Major and Trace Element Data for NIST SRM 610 and NIST SRM 612 Glass Reference Materials. Geostandards Newsletter. 21(1): 115-144

Raimondo T, Payne J, Wade B, Lanari P, Clark C, Hand M (2017) Trace element mapping by LA-ICP-MS: assessing geochemical mobility in garnet. Contributions to Mineralogy and Petrology. 172(4): DOI 10.1007/s00410-00017-01339-z

Russo RE, Mao X, Gonzalez JJ, Zorba V, Yoo J (2013) Laser Ablation in Analytical Chemistry. Analytical Chemistry. 85(13): 6162-6177

Russo RE, Mao XL, Liu C, Gonzalez J (2004) Laser assisted plasma spectrochemistry: laser ablation. J. Anal. At. Spectrom. 19(9): 1084-1089

Stead CV, Tomlinson EL, Kamber BS, Babechuk MG, McKenna CA (2017) Rare Earth Element Determination in Olivine by Laser Ablation - Quadrupole - ICP - MS: An Analytical Strategy and Applications. Geostand. Geoanal. Res. 41(2): 197-212

Stoll B, Jochum KP, Herwig K, Amini M, Flanz M, Kreuzburg B, Kuzmin D, Willbold M, Enzweiler J (2008) An automated iridium-strip heater for LA-ICP-MS bulk analysis of geological samples. Geostand. Geoanal. Res. 32(1): 5-26

Sylvester PJ (2008) Matrix effects in laser ablation ICP-MS. Laser Ablation ICP-MS in the Earth Sciences: Current Practices and Outstanding Issues (P. Sylvester, ed.). Mineralogical Association of Canada. Short Course Series. 40: 67-78 
Ubide T, McKenna CA, Chew DM, Kamber BS (2015) High-resolution LA-ICP-MS trace element mapping of igneous minerals: In search of magma histories. Chem. Geol. 409: $157-168$

Wang Z, Hattendorf B, Günther D (2006) Vaporization and ionization of laser ablation generated aerosols in an inductively coupled plasma mass spectrometer-implications from ion distribution maps. J. Anal. At. Spectrom. 21(11): 1143-1151

Wu S, Wang Y, Xu C, Yuan J (2016) Elemental Fractionation Studies of $193 \mathrm{~nm}$ ArF Excimer Laser Ablation System at High Spatial Resolution Mode. Chinese Journal of Analytical Chemistry. 44(7): 1035-1041

Wu S, Wang Y, Zhan X, Kronz A, Simon K, Xu C, Tian H (2016) Study on the Elemental Fractionation Effect of CGSG Reference Materials and the Related Within-Unit Homogeneity of Major and Trace Elements. Rock and Mineral Analysis. 35(6): 612-620

Zhang L, Ren Z-Y, Nichols ARL, Zhang Y-H, Zhang Y, Qian S-P, Liu J-Q (2014) Lead isotope analysis of melt inclusions by LA-MC-ICP-MS. J. Anal. At. Spectrom. 29(8): $1393-1405$ 


\section{Chapter 2.2 Laser Ablation Inductively Coupled Plasma Mass}

\section{Spectrometer: A quantification Strategy Based on Two Reference}

\section{Materials and Bulk Normalization as $100 \%$ (wt)}

Shitou $\mathrm{Wu}^{1,2}$, Yaping Wang ${ }^{2 *}$, Chunxue $\mathrm{Xu}^{2}$

${ }^{1}$ Geowissenschaftliches Zentrum, Göttingen Universität, Göttingen 37077, Germany

${ }^{2}$ National Research Center of Geoanalysis, Beijing 100037, China

\section{Graphical abstract}

The matrix effects among NIST, MPI-DING and USGS glasses were insignificant under the experimental conditions. A quantification strategy based on two reference materials (NIST610 and StHs6/80-G) and bulk normalization as $100 \%$ (wt) was proposed to reduce LA-ICP-MS transient signals, which eliminates the deficiencies encountered with using single reference material. The proposed strategy improved the analytical accuracy for LA-ICP-MS analysis

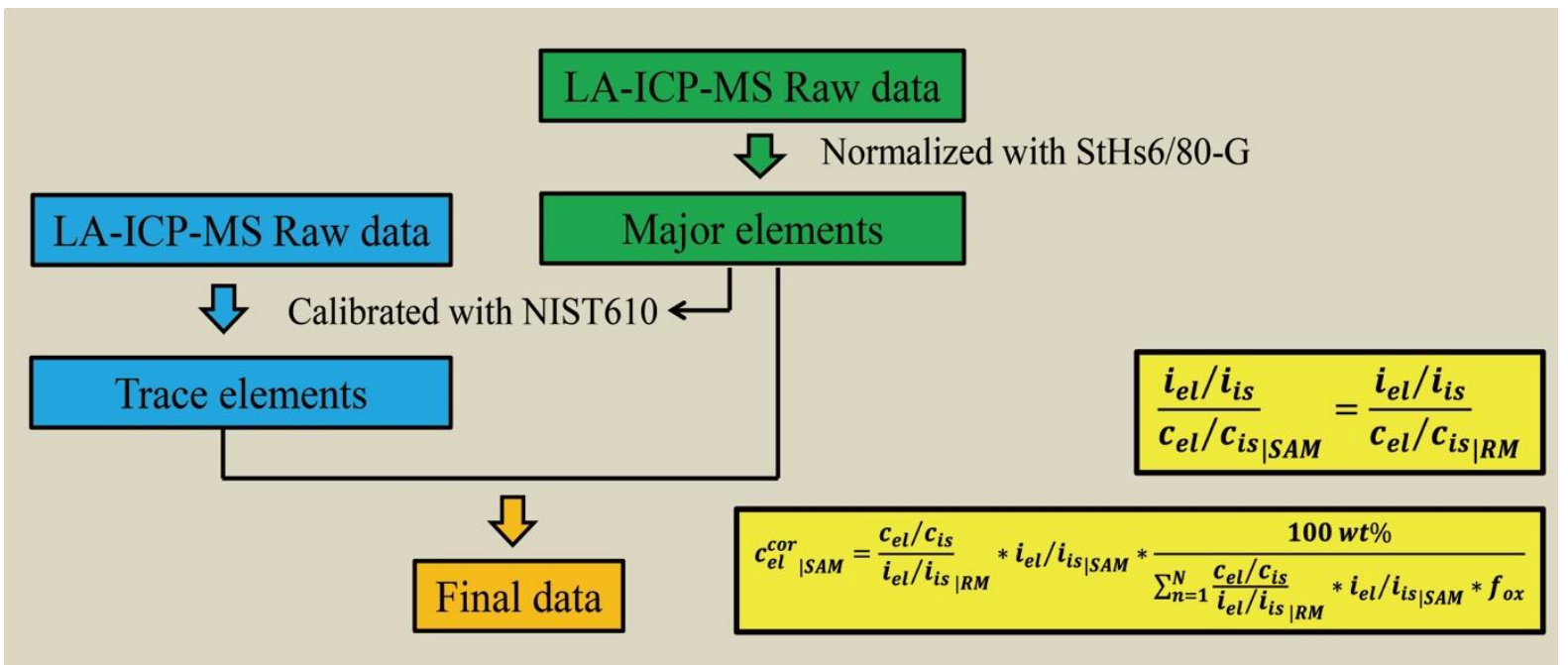


Abstract: Quantification strategy as an essential issue for laser ablation ICP-MS plays an important role in the guarantee of analytical accuracy. In this study, the reference value uncertainties of current available glass reference materials (including NIST, MPI-DING and USGS) as well as the matrix effects were systematically evaluated. The results illustrated that NIST610 was better than other glass reference materials from aspect of reference value uncertainty. The matrix effects among NIST, MPI-DING and USGS glasses were insignificant under the experimental conditions. The quantification strategy based on two reference materials (NIST610 and StHs6/80-G) and bulk normalization as $100 \%(w t)$ was proposed to reduce the LA-ICP-MS generated transient signals, which eliminates the deficiencies encountered with the quantification strategy using single reference material, such as the extreme low content or large uncertainty of some elements. The comparison of ML3B-G results obtained from three quantification strategies (single reference material NIST610, single reference material StHs6/80-G and two reference materials) illustrated that the proposed strategy improved the analytical accuracy. Three reference materials including BCR-2G, CGSG-2 and KL-2G were quantified using the proposed strategy, and almost all data matched well with reference values. The data reported in this study could supplement the reference value database for BCR-2G, CGSG-2 and KL2-G.

Key Words: Laser ablation inductively coupled plasma mass spectrometer; Quantification strategy; Reference value uncertainty, Matrix effect; Micro-analysis 


\subsubsection{Introduction}

Laser ablation-inductively coupled plasma-mass spectrometer (LA-ICP-MS) as a crucial micro-technique for chemical analysis provides some advantages including high spatial resolution ( $\mu \mathrm{m}$ scale), low detection limit $\left(\mathrm{ng} \mathrm{g}^{-1}\right)$ and rapid analysis ( $2 \mathrm{~min}$ per sample), and is widely used in analytical geochemistry (Koch and Günther, 2011; Liu et al., 2013; Russo et al., 2013; Wu et al., 2015). With the rapid development in instrument capability, more attention would be paid to this technique (Garbe-Schönberg and Müller, 2014; Wu et al., 2016; Zack and Hogmalm, 2016). Quantification strategy as an essential part for LA-ICP-MS could directly influence the data quality, and is an important research topic since the emergence of LA-ICP-MS (Liu et al., 2008; Miliszkiewicz et al., 2015). Quantification strategy mainly includes three aspects that needs to be corrected and calibrated: ICP mass response (using a solid external reference material or the solution calibration curve (Halicz and Gunther, 2004; Wälle et al., 2008), ablation mass amount during each analysis (based on an known internal standard (Longerich et al., 1996) or bulk normalization as 100\% (wt) (Liu et al., 2008)) and non-stoichiometric ablation behavior (matrix-matched calibration (Hu et al., 2011; Jochum et al., 2012) or correction of elemental fractionation (Horn et al., 2000; Chad Paton et al., 2010)). Currently available quantification strategies for LA-ICP-MS include single external reference material combined with internal standardization (Longerich et al.,, 1996; Norman et al., 1996), single external reference material combined with bulk normalization as 100\% (wt) (Gagnon et al., 2008; Guillong et al., 2005; Zhang et al., 2016), single external reference material combined with multiple internal standards (Jackson, 2008), multiple external reference materials without internal standardization (Liu et al., 2008).

The strategy of single external reference material combined with internal standardization was described by Longerich et al. (1996). This strategy is simple and practical. However, the concentration of internal standard should be known prior to LA-ICP-MS analysis, which led to complex procedure and increase the cost. Bulk normalization as $100 \%$ (wt) combined with a single external reference material was proposed to correct the ablation mass amount during 
each analysis, and the quantification could be achieved without knowing the concentration of internal standard. By using this strategy, Guillong et al. (2005) successfully quantified GSA-1G, GSC-1G, GSD-1G and GSE-1G. However the quantification strategy by using a single reference material has the deficiencies including extremely low content or large uncertainty for some elements. Jackson (2008) observed a linear relationship between elemental fractionation and analytical accuracy, and proposed the quantification strategy based on multiple internal standards. This strategy improved the accuracy of elements (like $\mathrm{Pb}$ and $\mathrm{Zn}$ ) that were heavily affected by elemental fractionations, while recent studies (Li et al., 2015; Wu et al., 2016) demonstrated the elemental fractionations induced by $193 \mathrm{~nm} \mathrm{ArF}$ excimer laser were insignificant. The quantification strategy by using multiple external reference materials without internal standardization was reported by Liu et al. (2008). This approach could correct the ablation mass amounts based on bulk normalization as 100\% (wt) combined with multiple external reference materials. However the use of multiple reference materials would increase the analytical workload, and without using internal standardization, it may result in a poor precision.

The reference value uncertainties of external reference materials directly influence the quality of analytical results (Luo et al., 2007), and it is controversial that whether there exist matrix effects among different glass substrates (synthetic glass and geological glass) or not (Czas et al., 2012; Jochum et al., 2014; Liu et al., 2008; Yuan et al., 2015; Yuan et al., 2011). In this study, the reference value uncertainties of different glass reference materials were systematically evaluated, and the matrix effects among NIST, MPI-DING and USGS glasses were investigated. A quantification strategy based on two external reference materials (NIST610 and StHs6/80-G) and bulk normalization as 100\% (wt) was proposed. The proposed quantification strategy could eliminate the deficiencies encountered with the strategy using single external reference material, such as non-matrix matching of NIST610 with geological glass and some elements with extreme low content or large uncertainty in StHs6/80-G. The comparison of the three quantification strategies (single reference material NIST610, single reference material StHs6/80-G and two reference materials) for quantifying 
ML3B-G was carried out. Three reference glasses including BCR-2G, CGSG-2 and KL2-G were quantified with the proposed strategy.

\subsubsection{Experimental}

\subsubsection{Instrumentation}

A $193 \mathrm{~nm}$ ArF excimer laser ablation system (RESOlution M-50, ASI, Australia) coupled to an Element 2 sector field ICP-MS (ThermoScientific, USA) were used for the data acquisition. The instrumental conditions were optimized to achieve the highest ${ }^{139}$ La intensity while keeping the $\mathrm{U} / \mathrm{Th} \approx 1$, oxide $(\mathrm{ThO} / \mathrm{Th})$ and secondary ion production $\left(\mathrm{Ca}^{2+} / \mathrm{Ca}^{+}\right)<0.5 \%$ by ablating NIST 612 in raster mode. The optimized instrumental conditions are summarized in Table 1.

Table1 Operation conditions of LA-ICP-MS system

\begin{tabular}{cccc}
\hline \multicolumn{2}{c}{ Laser ablation system } & \multicolumn{2}{c}{ ICP-MS } \\
\hline Laser type & ArF Excimer & ICP-MS & Element 2 \\
Wavelength & $193 \mathrm{~nm}$ & RF Power & $1500 \mathrm{~W}$ \\
Pulse width & $20 \mathrm{~ns}$ & Guard electrode & Floated \\
Energy density & $3.0 \mathrm{~J} / \mathrm{cm}^{2}$ & Coolant gas flow & $15.00 \mathrm{~L} / \mathrm{min}$ \\
Frequency & $5 \mathrm{~Hz}$ & Auxiliary gas flow & $1.00 \mathrm{~L} / \mathrm{min}$ \\
Ablation cell & Laurin Technic S-155 & Carrier gas flow & $0.95 \mathrm{~L} / \mathrm{min}$ \\
Spot size & $50 \mu \mathrm{m}$ & Segment duration & $10 \mathrm{~ms}$ \\
Ablation gas flow & $0.65 \mathrm{~L} / \mathrm{min}$ & Detector & Counting and analog \\
Ablation time & $35 \mathrm{~s}$ & Resolution $(\mathrm{M} / \Delta \mathrm{M})$ & Low $(\sim 300)$ \\
\hline
\end{tabular}

\subsubsection{Samples}

The samples used in this study include NIST reference materials (NIST610 and NIST612), MPI-DING reference materials (ATHO-G, StHs6/80-G, T1-G, ML3B-G, KL2-G, GOR128-G and GOR132-G), CGSG reference material (CGSG-2) and USGS reference materials (BIR-1G, BCR-2G, BHVO-1G and GSD-1G). NIST612 was used for optimizing instrumental conditions. NIST610 and StHs6/80-G were used as external reference materials. In addition, NIST610 was used for the correction of instrument drift. GSD-1G was used for data quality control. The reference values and uncertainties were citied from literatures (Jochum et al., 2006; Jochum et al., 2011) and GeoReM database (Jochum and Nohl, 2008) (http://georem. mpch-mainz.gwdg.de/). All the samples were mounted in the epoxy resin, and 
polished to a flatness surface $(1 \mu \mathrm{m})$. The samples were cleaned with ethanol, and then dried using pressured $\mathrm{N}_{2}$ prior to LA-ICP-MS analysis. The analysis sequence is as follows: external reference material (NIST610), external reference material (StHs6/80-G), quality control sample (GSD-1G), unknown samples (10), external reference material (NIST610), external reference material (StHs6/80-G), quality control sample (GSD-1G) and unknown samples (10).

\subsubsection{Quantification strategy}

The approach of bulk normalization as $100 \%$ (wt) allows quantification without knowing the concentration of internal standard, which simplifies the analytical procedure. The prerequisite for the application of bulk normalization as $100 \%(w t)$ is to accuracy analyze as more elements as possible, which could reduce the uncertainties introduced by the bulk normalization. In this study, 51 elements were measured, except halogen and platinum group metals. The sum of elemental oxides is $>99 \%$ ( $w t$ ) (Table 2). The element concentrations need to be conversed to oxide concentrations (by multiplying oxide coefficient). The assumption of valence state for the elements having multiple valence, especially with high concentration (>1\%), may influence the bulk normalization. Here we assumed $\mathrm{Fe}^{2+} / \mathrm{Fe}^{3+}$ as 1 .

Table 2 Sum of elemental oxides of the investigated reference materials

\begin{tabular}{cccc}
\hline Name & Sum of oxide $(\%)$ & Name & Sum of oxide $(\%)$ \\
\hline NIST610 & 99.190 & GOR128-G & 100.254 \\
NIST612 & 99.980 & GOR132-G & 100.000 \\
ATHO-G & 100.079 & BCR-2G & 99.637 \\
StHs6/80-G & 100.203 & BIR-1G & 100.014 \\
ML3B-G & 99.120 & BHVO-2G & 99.781 \\
KL2-G & 99.169 & GSD-1G & 99.743 \\
T1-G & 99.700 & & \\
\hline
\end{tabular}

The implementation of the proposed strategy incorporates two steps. (1) With StHs6/80-G as external reference material and by combination with bulk normalized as $100 \%(w t)$, the major element concentrations were calculated by Eq.(3); (2) By using NIST610 as external reference material and $\mathrm{Ca}$ as internal standard (concentration of $\mathrm{Ca}$ is collected from step (1)), 
the trace element concentrations were calculated based on Eq.(1). The configuration for the implementation of the proposed strategy is shown in Fig.1.

The strategy of single external reference material combined with internal standardization reported by Longerich et al. (1996) is shown in Eq. (1)

$$
\frac{i_{e l} / i_{i s}}{c_{e l} / c_{i s} \mid S A M}=\frac{i_{e l} / i_{i S}}{c_{e l} / c_{i s} \mid R M}
$$

The bulk normalization as $100 \%(w t)$ is shown in Eq. (2)

$$
\sum_{n=1}^{N} c_{e l}^{n} * f_{o x}^{n}=100 w t \%
$$

The Eq. (1) was substituted into (2) to obtain Eq. (3):

$$
c_{e l}^{c o r}{ }_{\mid S A M}=\frac{c_{e l} / c_{i S}}{i_{e l} / i_{i s}}{ }_{\mid R M} * i_{e l} / i_{i S_{\mid S A M}} * \frac{100 w t \%}{\sum_{n=1}^{N} \frac{c_{e l} / c_{i S}}{i_{e l} / i_{i S}} * i_{e l} / i_{i S \mid S A M} * f_{o x}}
$$

Where $i$ and $c$ represent the signal intensity (cps) and concentration; $e l$ and $i$ s represent the target element and internal standard; $o x$ represents the element oxide; $S A M$ and $R M$ represent sample and reference material; $f$ represents the oxide coefficient

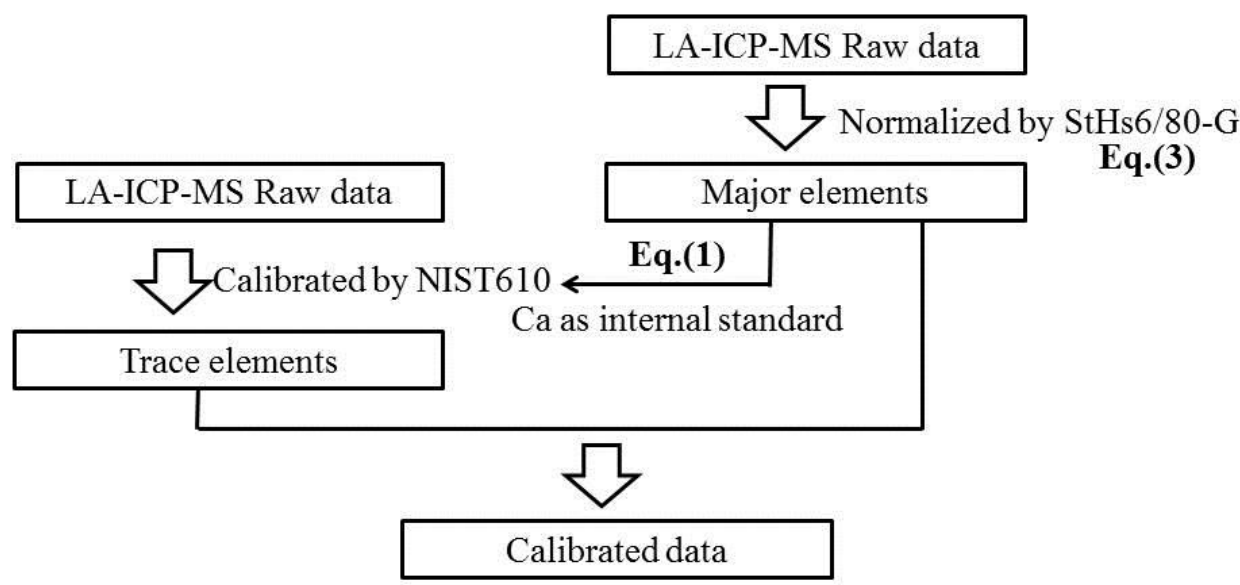

Fig.1 Configuration of quantification strategy based on two reference materials and bulk normalization as $100 \%$ (wt) 
The proposed quantification strategy was implemented in Iolite3.0 (Paton et al., 2011), including background subtraction, instrument drift correction and transient signal reduction. The bulk normalization (Eq. (3)) was accomplished with the author-edited data reduction scheme. In practical, the implementation of this strategy is as follows. The signal intensity was firstly subtracted from the background (gas blank), and then normalized to internal standard $(\mathrm{Ca})$. The intensity ratio was corrected for the instrument drift by using multiple analysis of NIST610 (linear interpolation). According to Eq. (3) and using StHs6/80-G as external reference material, the major element concentrations were calculated. The bulk normalization as $100 \%$ (wt) was calculated based on 51 measured elements. The trace elements were quantified with NIST610 as external reference material and Ca as internal standard (the concentration of Ca was collected from step (1)). The concentrations of major elements (from step (1)) and trace elements (from step (2)) were the final data.

\subsubsection{Results and discussions}

\subsubsection{Uncertainty of reference values}

The reference value uncertainties of external reference materials would directly propagate to analytical results, so that the analytical results are severely influenced by the uncertainty of external reference material. Currently available reference materials for LA-ICP-MS include NIST, MPI-DING and USGS glasses. Figure 2 shows the comparison of reference value uncertainties of NIST610, MPI-DING (ATHO-G, StHs6/80-G, T1-G, GOR132-G and GOR128-G) and USGS (BCR-2G and GSD-1G). The reference value uncertainties were collected from GeoReM database (http://georem. mpch-mainz.gwdg.de/). 


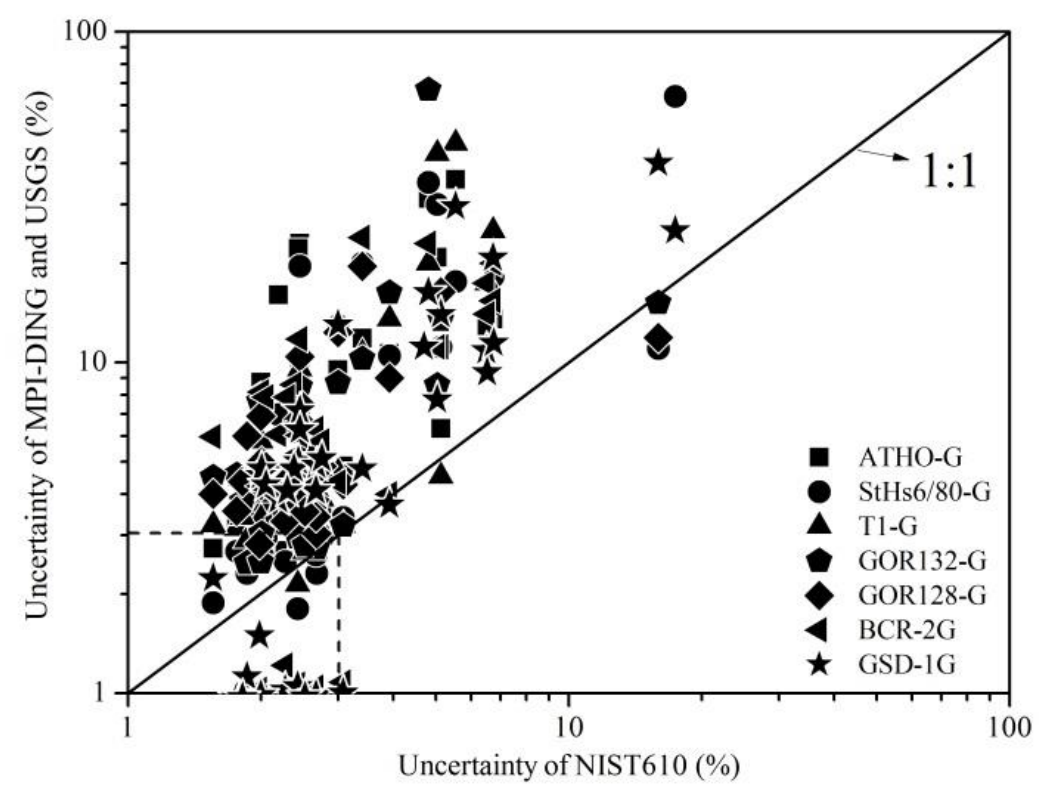

Fig. 2 Comparison of reference value uncertainty between NIST610 and MPI-DING, USGS reference materials

The results in Fig.2 illustrated that the uncertainties of NIST610 were smaller than that those of MPI-DING and USGS glasses. The relative small uncertainties of NIST610 could be interpreted from two aspects. One was that the value certification of NIST610 was thoroughly carried out in previous studies, and accepted as primary reference materials for LA-ICP-MS calibration. The other was that NIST610 has high abundance of trace elements. Commonly elements with high abundance are easily certified, thus their uncertainties are relative small. Luo et al. (2007) demonstrated that the main source of LA-ICP-MS analytical uncertainty was derived from the uncertainty of external reference material. In summary, analytical results were better when using NIST610 as external reference material than MPI-DING and USGS glasses.

\subsubsection{Matrix effects}

The matrix effects between samples and reference materials may strongly influence the analytical accuracy of LA-ICP-MS, especially where the matrix-matched calibration cannot be achieved (Sylvester, 2008). The matrix effects could steam from ablation procedure (Luo et al., 2015; Tang et al., 2015), aerosol transportation (Kovacs and Günther, 2008) and ICP 
ionization process (Kroslakova and Günther, 2007). Matrix effect between synthetic glass (NIST series) and geological glass (MPI-DING and USGS) has been debated in previous studies. Liu et al. (2008) observed serious matrix effects between NIST610 and MPI-DING glasses, while Yuan et al. (2011) demonstrated that the matrix effects between synthetic and geological glasses were insignificant. In this study, based on the quantification strategy described by Longerich et al. (1996), The normalized intensity (normalized to Ca) and concentration (normalized to $\mathrm{Ca}$ ) were plotted, and the linear coefficients was used to evaluate the matrix effects among different glass substrates (as shown in Fig.3). The elemental fractionation that is specified to a certain substrate might be one of sources for the matrix effects. Our previous study (Wu et al., 2016) illustrated that the elemental fractionation of glasses induced by $193 \mathrm{~nm}$ laser was insignificant as the ablation depth was smaller than spot size. All the data were collected when ablation depth was smaller than spot size.

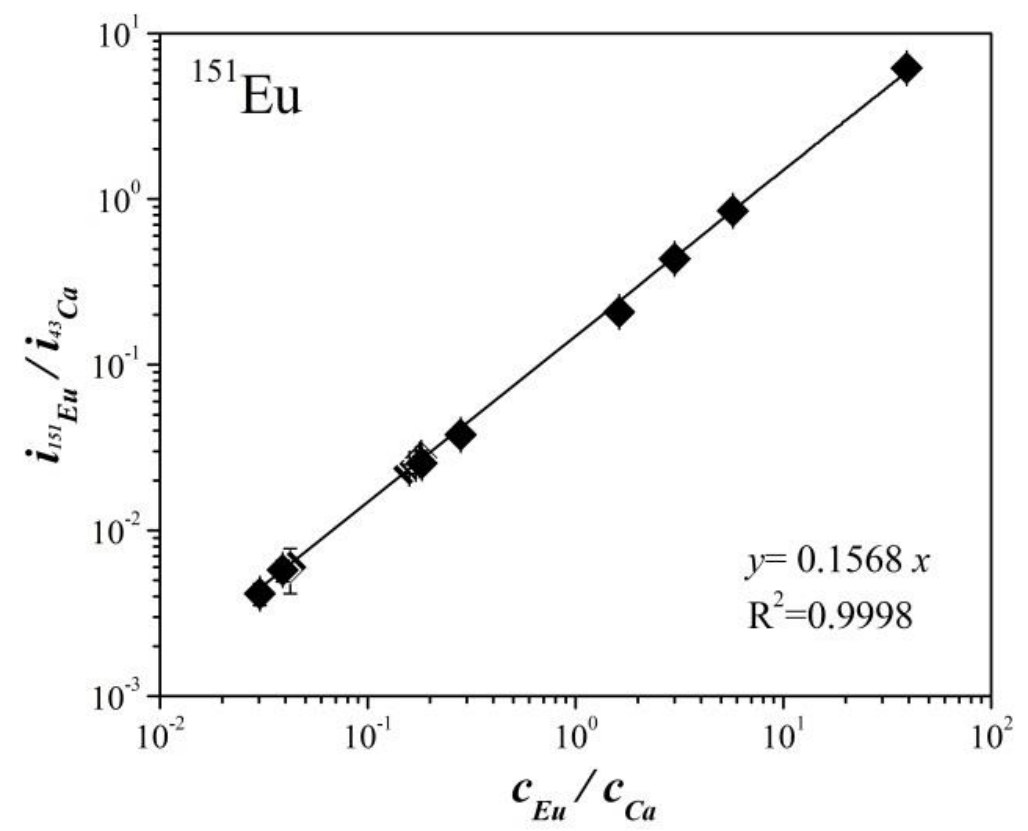

Fig.3 The relationship of intensity of ${ }^{151} \mathrm{Eu}$ (normalized to ${ }^{43} \mathrm{Ca}$ ) and content of $\mathrm{Eu}$ (normalized to $\mathrm{Ca}$ )

The matrix effects among NIST, MPI-DING and USGS series glass were systematically evaluated. The results illustrated that the linear coefficients of most elements were in the 
range of $0.9980-0.9999$, demonstrating that the matrix effects were insignificant. Several elements including P, Fe, V, Cu and Zn showed the linear coefficients of 0.9900-0.9980. This was probably caused by the potential imprecision of reference values. Some of those elements were given as information values or had relative large uncertainties. In summary, the matrix effects between synthetic and geological glasses were insignificant under our experimental conditions. The calibration of synthetic glass could use geological glass as external reference material, and vice versa.

\subsubsection{Quantification strategy based on two reference materials and bulk normalization as} $100 \%(\mathrm{wt})$

The major element compositions of NIST series glass are significantly different from those of geological glasses. For example, concentration of $\mathrm{MgO}$ in NIST610 is only $716 \mu \mathrm{g} \mathrm{g}^{-1}$, while the concentrations of $\mathrm{MgO}$ in most geological glass are higher than $2 \%(w t)$, so that the analytical results of some major element may have large deviations when using NIST610 as external reference material. Several elements in geological glass have very low concentration or large uncertainty, for instance, the concentration of Lu in StHs6/80-G is only $0.168 \mu \mathrm{g} \mathrm{g}^{-1}$, which is not usable as the external reference material in the quantification strategies for these elements. Three quantification strategies (including single reference material NIST610, single reference material StHs6/80-G and two reference materials) were compared for quantifying ML3B-G (Fig.4). The grey zone represents the reference value uncertainty. The error bar is given as 1 standard deviation $(\mathrm{n}=10)$. The ordinate shows the relative deviation $(\%)$. The calculation of the relative deviation (\%) is shown by Eq. (4)

Relative deviation $(\%)=\frac{c_{m e a r}-c_{R V}}{c_{R V}} \times 100 \%$

The results as shown in Fig.4 illustrated that $\mathrm{TiO}_{2}, \mathrm{MgO}$ and $\mathrm{K}_{2} \mathrm{O}$ had relative large deviation when using NIST610 as external reference material, while several trace elements including $\mathrm{Nb}, \mathrm{Mo}, \mathrm{Sn}, \mathrm{Tm}, \mathrm{Lu}$ and $\mathrm{W}$ showed large deviation (>10\%) when using StHs6/80-G as external reference material. The quantification strategy based on two reference materials and 
bulk normalization as $100 \%$ (wt) efficiently eliminated the deficiencies encountered with the strategy using single reference material. Almost all analytical results obtained from the proposed strategy were in the range of reference value uncertainty, which demonstrated that the proposed strategy improved the analytical accuracy.

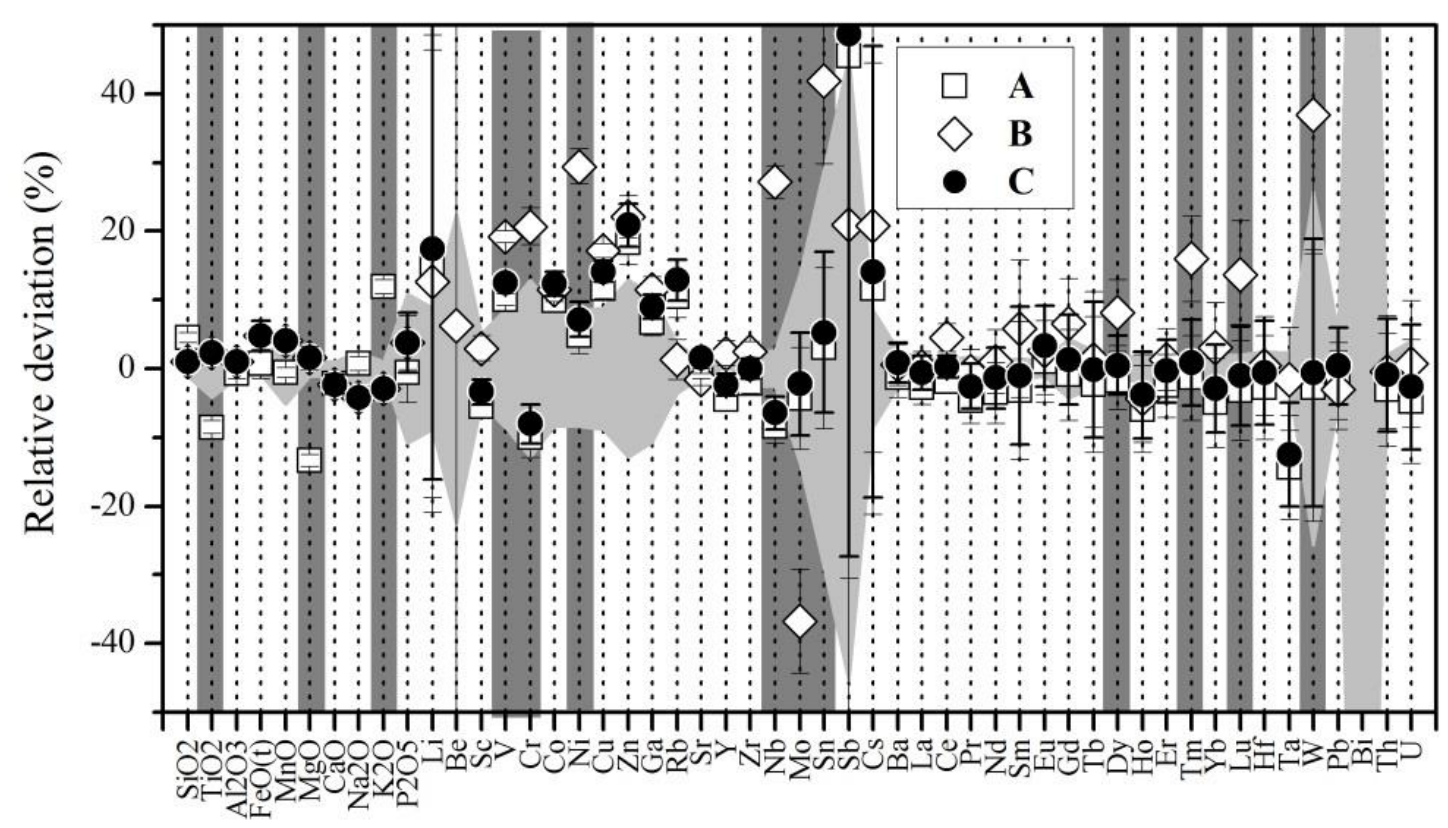

Fig. 4 Comparison of calibrated data of ML3B-G (A) NIST610 normalization strategy, (B) StHs6/80-G normalization strategy, (C) two steps normalization strategy.

\subsubsection{Analysis of MPI-DING, USGS and CGSG glass reference materials}

The major and trace elements in BCR-2G, CGSG-2 and KL2-G were quantified using the proposed strategy (Fig.5 and Table 3). The grey zone represents the reference value uncertainty, and the error bars are given as 1 standard deviation $(n=10)$. The reference values and uncertainties of BCR-2G and KL2-G were cited from GeoReM database, and CGSG-2 was cited from reference (Jochum and Enzweiler, 2014). 


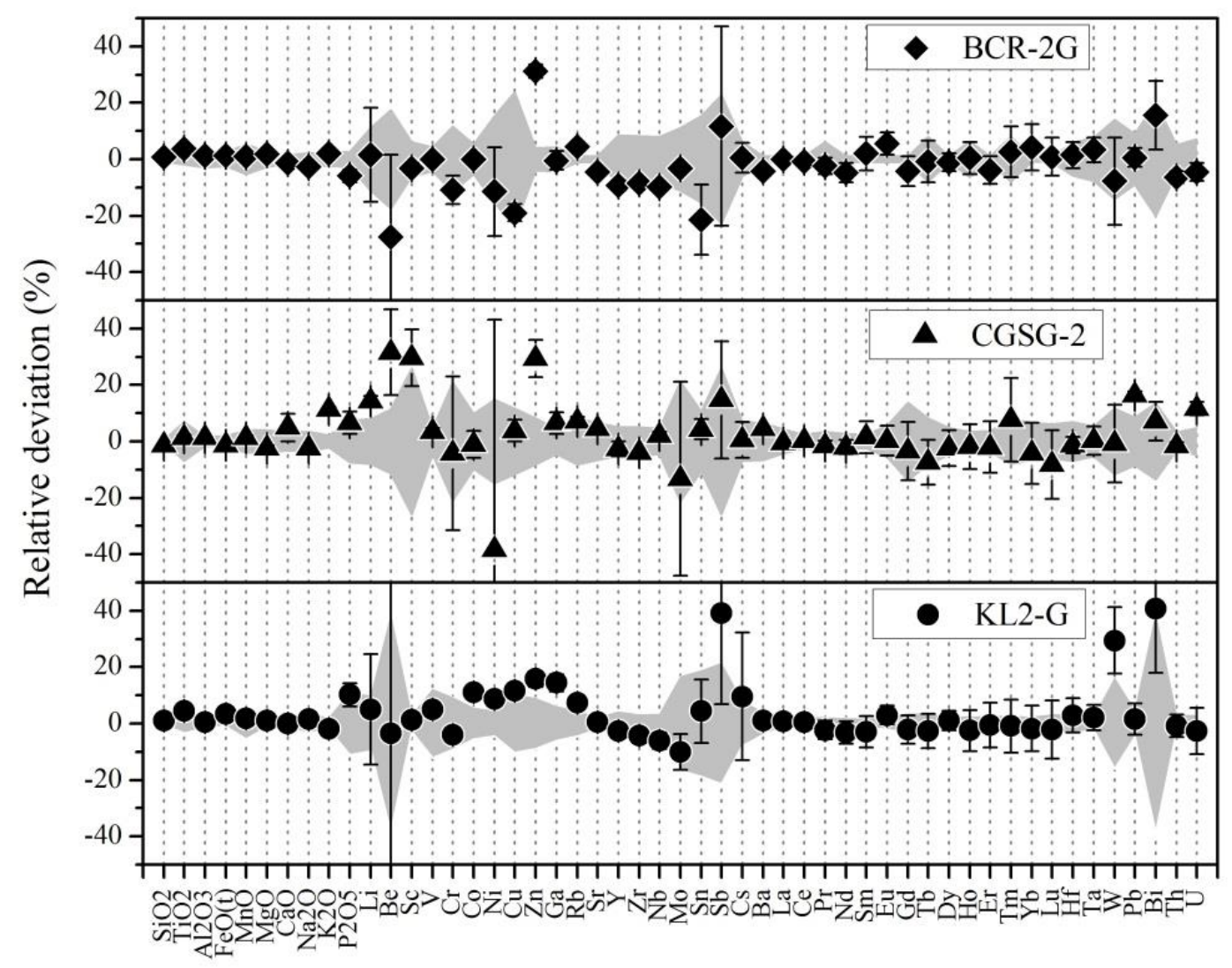

Fig. 5 Calibrated data of BCR-2G, CGSG-2 and KL2-G using the proposed strategy

It can be seen from Fig. 5 and Table 3 that analytical results of most major and trace elements matched well with reference values within $95 \%$ confidence, which demonstrated that the proposed strategy was approvingly applicable for the reduction of LA-ICP-MS transient signal. Several elements including $\mathrm{Zn}$ in BCR-2G, Be, Zn in CGSG-2 and Pb in KL2-G were out of the range of reference value uncertainty. This was probably attributed to the imprecise of reference values or the potential heterogeneity. The data reported in this study could supplement the reference value database for BCR-2G, CGSG-2 and KL2-G. 
Chapter 2.2 Laser Ablation Inductively Coupled Plasma Mass Spectrometer: A quantification Strategy Based on Two Reference Materials and Bulk Normalization as 100 \% (wt)

Table 3 Reference values and measured data of BCR-2G, CGSG-2 and KL2-G

\begin{tabular}{|c|c|c|c|c|c|c|c|c|c|}
\hline & \multicolumn{3}{|c|}{ BCR-2G } & \multicolumn{3}{|c|}{ CGSG-2 } & \multicolumn{3}{|c|}{ KL2-G } \\
\hline & $\begin{array}{c}\text { GeoReM } \\
\text { data }\end{array}$ & $\begin{array}{c}\text { Measured } \\
\text { data }\end{array}$ & $\begin{array}{c}\text { RSD } \\
(\%)\end{array}$ & $\begin{array}{c}\text { Jochum'dat } \\
\text { a } \\
\end{array}$ & $\begin{array}{c}\text { Measured } \\
\text { data }\end{array}$ & $\begin{array}{l}\text { RSD } \\
(\%)\end{array}$ & $\begin{array}{c}\text { GeoReM } \\
\text { data }\end{array}$ & $\begin{array}{c}\text { Measured } \\
\text { data }\end{array}$ & $\begin{array}{c}\text { RSD } \\
(\%) \\
\end{array}$ \\
\hline $\mathrm{SiO}_{2}$ & 54.4 & 54.8 & 0.32 & 54.3 & 53.5 & 0.22 & 50.3 & 50.8 & 0.44 \\
\hline $\mathrm{TiO}_{2}$ & 2.27 & 2.35 & 0.53 & 0.59 & 0.598 & 1.06 & 2.56 & 2.67 & 0.74 \\
\hline $\mathrm{A}_{12} \mathrm{O}_{3}$ & 13.4 & 13.6 & 0.41 & 20.8 & 21.1 & 0.32 & 13.3 & 13.4 & 0.34 \\
\hline $\mathrm{FeO} \_\mathrm{t}$ & 12.4 & 12.6 & 0.91 & 6.76 & 6.66 & 0.77 & 10.7 & 11.1 & 0.78 \\
\hline $\mathrm{MnO}$ & 0.19 & 0.192 & 0.79 & 0.13 & 0.132 & 0.79 & 0.165 & 0.168 & 1.14 \\
\hline $\mathrm{MgO}$ & 3.56 & 3.62 & 0.93 & 0.87 & 0.849 & 0.93 & 7.34 & 7.42 & 1.15 \\
\hline $\mathrm{CaO}$ & 7.06 & 6.97 & 1.09 & 1.7 & 1.79 & 4.90 & 10.9 & 10.9 & 1.29 \\
\hline $\mathrm{Na}_{2} \mathrm{O}$ & 3.23 & 3.14 & 0.79 & 6.55 & 6.39 & 0.42 & 2.35 & 2.39 & 0.82 \\
\hline $\mathrm{K}_{2} \mathrm{O}$ & 1.74 & 1.78 & 0.87 & 6.95 & 7.74 & 0.76 & 0.48 & 0.472 & 1.35 \\
\hline $\mathrm{P}_{2} \mathrm{O}_{5}$ & 0.37 & 0.348 & 2.28 & 0.093 & 0.099 & 3.97 & 0.232 & 0.256 & 4.18 \\
\hline $\mathrm{Li}$ & 9 & 9.14 & 16.59 & 411 & 470 & 1.65 & 5.1 & 5.36 & 19.62 \\
\hline $\mathrm{Be}$ & 2.3 & 1.67 & 28.91 & 16.9 & 22.2 & 15.22 & 0.88 & 0.85 & 73.74 \\
\hline $\mathrm{Sc}$ & 33 & 32 & 1.71 & 4.34 & 5.63 & 9.94 & 31.8 & 32.2 & 2.52 \\
\hline $\mathrm{V}$ & 425 & 425 & 0.84 & 206 & 213 & 1.32 & 309 & 325 & 0.83 \\
\hline $\mathrm{Cr}$ & 17 & 15.2 & 5.11 & 8.1 & 7.76 & 27.20 & 294 & 283 & 1.44 \\
\hline Co & 38 & 38 & 1.52 & 6.15 & 6.08 & 4.74 & 41.2 & 45.8 & 2.05 \\
\hline $\mathrm{Ni}$ & 13 & 11.5 & 15.75 & 4.48 & 2.76 & 81.45 & 112 & 122 & 1.94 \\
\hline $\mathrm{Cu}$ & 21 & 17 & 3.15 & 21.2 & 22 & 3.90 & 87.9 & 98.2 & 2.01 \\
\hline $\mathrm{Zn}$ & 125 & 164 & 2.46 & 124 & 160 & 6.55 & 110 & 127 & 2.81 \\
\hline $\mathrm{Ga}$ & 23 & 22.9 & 3.22 & 38 & 40.5 & 3.80 & 20 & 22.9 & 3.01 \\
\hline $\mathrm{Rb}$ & 47 & 49.1 & 1.13 & 124 & 133 & 1.48 & 8.7 & 9.34 & 1.74 \\
\hline $\mathrm{Sr}$ & 342 & 327 & 0.82 & 1161 & 1214 & 0.38 & 356 & 358 & 0.68 \\
\hline $\mathrm{Y}$ & 35 & 31.7 & 1.87 & 28.5 & 27.8 & 2.57 & 25.4 & 24.7 & 1.59 \\
\hline $\mathrm{Zr}$ & 184 & 168 & 0.75 & 1424 & 1367 & 0.53 & 152 & 146 & 0.76 \\
\hline $\mathrm{Nb}$ & 12.5 & 11.3 & 0.94 & 69.8 & 71.2 & 1.38 & 15 & 14.1 & 2.92 \\
\hline Mo & 270 & 262 & 1.04 & 1.23 & 1.07 & 34.42 & 3.6 & 3.24 & 6.25 \\
\hline $\mathrm{Sn}$ & 2.6 & 2.05 & 12.47 & 11.1 & 11.6 & 3.61 & 1.54 & 1.61 & 11.24 \\
\hline $\mathrm{Sb}$ & 0.35 & 0.391 & 35.26 & 1.62 & 1.86 & 20.87 & 0.14 & 0.195 & 32.37 \\
\hline Cs & 1.16 & 1.17 & 5.32 & 2.02 & 2.03 & 6.29 & 0.115 & 0.126 & 22.71 \\
\hline $\mathrm{Ba}$ & 683 & 654 & 1.72 & 390 & 408 & 0.80 & 123 & 124 & 1.34 \\
\hline $\mathrm{La}$ & 24.7 & 24.7 & 1.40 & 160 & 159 & 0.91 & 13.1 & 13.2 & 1.31 \\
\hline $\mathrm{Ce}$ & 53.3 & 52.9 & 1.44 & 256 & 256 & 0.71 & 32.4 & 32.6 & 1.23 \\
\hline $\operatorname{Pr}$ & 6.7 & 6.54 & 2.97 & 23.2 & 22.8 & 1.83 & 4.6 & 4.49 & 3.25 \\
\hline $\mathrm{Nd}$ & 28.9 & 27.5 & 3.46 & 74.6 & 73.1 & 2.55 & 21.6 & 20.9 & 4.00 \\
\hline $\mathrm{Sm}$ & 6.59 & 6.72 & 5.86 & 9.75 & 9.9 & 5.70 & 5.54 & 5.38 & 5.55 \\
\hline $\mathrm{Eu}$ & 1.97 & 2.08 & 3.94 & 2.48 & 2.49 & 5.26 & 1.92 & 1.98 & 3.29 \\
\hline $\mathrm{Gd}$ & 6.71 & 6.43 & 5.21 & 6.86 & 6.62 & 10.34 & 5.92 & 5.79 & 5.06 \\
\hline $\mathrm{Tb}$ & 1.02 & 1.01 & 7.38 & 0.97 & 0.898 & 8.00 & 0.89 & 0.866 & 6.02 \\
\hline Dy & 6.44 & 6.38 & 3.18 & 4.96 & 4.85 & 6.35 & 5.22 & 5.27 & 3.39 \\
\hline Но & 1.27 & 1.28 & 5.73 & 0.9 & 0.883 & 7.85 & 0.961 & 0.937 & 7.24 \\
\hline $\mathrm{Er}$ & 3.7 & 3.55 & 4.90 & 2.66 & 2.61 & 9.24 & 2.54 & 2.53 & 8.01 \\
\hline $\mathrm{Tm}$ & 0.51 & 0.524 & 8.94 & 0.41 & 0.441 & 14.78 & 0.331 & 0.328 & 9.40 \\
\hline $\mathrm{Yb}$ & 3.39 & 3.54 & 8.14 & 2.76 & 2.65 & 10.84 & 2.1 & 2.06 & 8.03 \\
\hline $\mathrm{Lu}$ & 0.503 & 0.508 & 6.67 & 0.42 & 0.386 & 12.11 & 0.285 & 0.279 & 10.31 \\
\hline $\mathrm{Hf}$ & 4.84 & 4.91 & 4.74 & 35.4 & 34.9 & 2.80 & 3.93 & 4.04 & 6.06 \\
\hline $\mathrm{Ta}$ & 0.78 & 0.806 & 4.42 & 2.08 & 2.09 & 4.96 & 0.961 & 0.982 & 4.53 \\
\hline $\mathrm{W}$ & 0.5 & 0.461 & 15.57 & 1.53 & 1.52 & 13.67 & 0.37 & 0.479 & 11.86 \\
\hline $\mathrm{Pb}$ & 11 & 11.1 & 3.33 & 129 & 150 & 1.26 & 2.07 & 2.1 & 5.62 \\
\hline $\mathrm{Bi}$ & 0.05 & 0.058 & 12.25 & 1.11 & 1.19 & 6.90 & 0.036 & 0.051 & 22.95 \\
\hline $\mathrm{Th}$ & 5.9 & 5.52 & 2.08 & 75.3 & 74.1 & 1.37 & 1.02 & 1.01 & 3.92 \\
\hline $\mathrm{U}$ & 1.69 & 1.62 & 3.17 & 13.7 & 15.3 & 2.32 & 0.548 & 0.533 & 8.21 \\
\hline
\end{tabular}

GeoReM data means the data are cited from GeoReM database; Jochum'data means the data are cited from

publication (Jochum and Enzweiler, 2014) 


\subsubsection{Conclusions}

In this study, the reference value uncertainties in current available glass reference materials were evaluated. The results illustrated that uncertainties of NIST610 were better than that of other glass reference materials. The normalized intensity (normalized to $\mathrm{Ca}$ ) and concentration (normalized to $\mathrm{Ca}$ ) were plotted, and the linear coefficient was used to evaluate the matrix effects among different reference materials (NIST, MPI-DING and USGS glasses). The results illustrated that the matrix effects were insignificant under this experimental conditions. The quantification strategy based on two reference materials and bulk normalization as $100 \%$ (wt) was proposed and this strategy efficiently eliminated the deficiencies encountered with the strategy using single reference material, such as extreme low concentration or large uncertainty for some elements. Three quantification strategies, including single reference material NIST610, single reference material StHs6/80-G and two reference materials were used for quantifying ML3B-G. The results illustrated that the proposed strategy improved the analytical accuracy. Three reference materials including BCR-2G, CGSG-2 and KL2-G were quantified using this strategy, and almost all data matched well with reference values, which demonstrated the applicability of the proposed strategy. 


\section{References}

Czas J, Jochum KP, Stoll B, Weis U, Yang QC, Jacob DE, Andreae MO (2012) Investigation of matrix effects in $193 \mathrm{~nm}$ laser ablation-inductively coupled plasma-mass spectrometry analysis using reference glasses of different transparencies. Spectroc. Acta Pt. B-Atom. Spectr. 78: 20-28

Gagnon JE, Fryer BJ, Samson IM, Williams-Jones AE (2008) Quantitative analysis of silicate certified reference materials by LA-ICPMS with and without an internal standard. J. Anal. At. Spectrom. 23(11): 1529-1537

Garbe-Schönberg D, Müller S (2014) Nano-particulate pressed powder tablets for LA-ICP-MS. J. Anal. At. Spectrom. 29 (6): 990-1000

Guillong M, Hametner K, Reusser E, Wilson SA, Günther D (2005) Preliminary Characterisation of New Glass Reference Materials (GSA-1G, GSC-1G, GSD-1G and GSE-1G) by Laser Ablation-Inductively Coupled Plasma-Mass Spectrometry Using 193 nm, $213 \mathrm{~nm}$ and $266 \mathrm{~nm}$ Wavelengths. Geostand. Geoanal. Res. 29(3): 315-331

Halicz L, Gunther D (2004) Quantitative analysis of silicates using LA-ICP-MS with liquid calibration. J. Anal. At. Spectrom. 19(12): 1539-1545

Horn I, Rudnick RL, McDonough WF (2000) Precise elemental and isotope ratio determination by simultaneous solution nebulization and laser ablation-ICP-MS: application to U-Pb geochronology. Chem. Geol. 164(3-4): 281-301

Hu ZC, Liu YS, Chen L, Zhou LA, Li M, Zong KQ, Zhu LY, Gao S (2011) Contrasting matrix induced elemental fractionation in NIST SRM and rock glasses during laser ablation ICP-MS analysis at high spatial resolution. J. Anal. At. Spectrom. 26(2): 425-430 
Jackson SE (2008) Calibration strategies for elemental analysis by LA-ICP-MS. Laser Ablation ICP-MS in the Earth Sciences: Current Practices and Outstanding Issues (P. Sylvester, ed.). Mineralogical Association of Canada. Short Course Series. 40: 169-188

Jochum KP, Enzweiler J: Reference Materials in Geochemical and Environmental Research: Treatise on Geochemistry (Second Edition); Elsevier, Oxford 2014, 43-70

Jochum KP, Nohl U (2008) Reference materials in geochemistry and environmental research and the GeoReM database. Chem. Geol. 253(1-2): 50-53

Jochum KP, Scholz D, Stoll B, Weis U, Wilson SA, Yang QC, Schwalb A, Borner N, Jacob DE, Andreae MO (2012) Accurate trace element analysis of speleothems and biogenic calcium carbonates by LA-ICP-MS. Chem. Geol. 318: 31-44

Jochum KP, Stoll B, Herwig K, Willbold M, Hofmann AW, Amini M, Aarburg S, Abouchami W, Hellebrand E, Mocek B, Raczek I, Stracke A, Alard O, Bouman C, Becker S, Dücking M, Brätz H, Klemd R, de Bruin D, Canil D, Cornell D, de Hoog C-J, Dalpé C, Danyushevsky L, Eisenhauer A, Gao Y, Snow JE, Groschopf N, Günther D, Latkoczy C, Guillong M, Hauri EH, Höfer HE, Lahaye Y, Horz K, Jacob DE, Kasemann SA, Kent AJR, Ludwig T, Zack T, Mason PRD, Meixner A, Rosner M, Misawa K, Nash BP, Pfänder J, Premo WR, Sun WD, Tiepolo M, Vannucci R, Vennemann T, Wayne D, Woodhead JD (2006) MPI-DING reference glasses for in situ microanalysis: New reference values for element concentrations and isotope ratios. Geochemistry, Geophysics, Geosystems. 7(2): $1-44$

Jochum KP, Stoll B, Weis U, Jacob DE, Mertz-Kraus R, Andreae MO (2014) Non-Matrix-Matched Calibration for the Multi-Element Analysis of Geological and 
Environmental Samples Using $200 \mathrm{~nm}$ Femtosecond LA-ICP-MS: A Comparison with Nanosecond Lasers. Geostand. Geoanal. Res. 38(3): 265-292

Jochum KP, Weis U, Stoll B, Kuzmin D, Yang QC, Raczek I, Jacob DE, Stracke A, Birbaum K, Frick DA, Günther D, Enzweiler J (2011) Determination of Reference Values for NIST SRM 610-617 Glasses Following ISO Guidelines. Geostand. Geoanal. Res. 35(4): 397-429

Koch J, Günther D (2011) Review of the State-of-the-Art of Laser Ablation Inductively Coupled Plasma Mass Spectrometry. Appl. Spectrosc. 65(5): 155A-162A

Kovacs R, Günther D (2008) Influence of transport tube materials on signal response and drift in laser ablation-inductively coupled plasma-mass spectrometry. J. Anal. At. Spectrom. 23(9): 1247-1252

Kroslakova I, Günther D (2007) Elemental fractionation in laser ablation-inductively coupled plasma-mass spectrometry: evidence for mass load induced matrix effects in the ICP during ablation of a silicate glass. J. Anal. At. Spectrom. 22(1): 51-62

Li Z, Hu Z, Liu Y, Gao S, Li M, Zong K, Chen H, Hu S (2015) Accurate determination of elements in silicate glass by nanosecond and femtosecond laser ablation ICP-MS at high spatial resolution. Chem. Geol. 400: 11-23

Liu YS, Hu ZC, Gao S, Gunther D, Xu J, Gao CG, Chen HH (2008) In situ analysis of major and trace elements of anhydrous minerals by LA-ICP-MS without applying an internal standard. Chem. Geol. 257(1-2): 34-43

Liu YS, Hu ZC, Li M, Gao S (2013) Applications of LA-ICP-MS in the elemental analyses of geological samples. Chinese Science Bulletin. 58(32): 3863-3878 
Longerich HP, Jackson SE, Günther D (1996) Inter-laboratory note. Laser ablation inductively coupled plasma mass spectrometric transient signal data acquisition and analyte concentration calculation. J. Anal. At. Spectrom. 11(9): 899-904

Luo T, Wang Y, Hu Z, Günther D, Liu Y, Gao S, Li M, Hu SH (2015) Further investigation into ICP-induced elemental fractionation in LA-ICP-MS using a local aerosol extraction strategy. J. Anal. At. Spectrom. 30(4): 941-949

Luo Y, Gao S, Longerich HP, Gunther D, Wunderli S, Yuan HL, Liu XM (2007) The uncertainty budget of the multi-element analysis of glasses using LA-ICP-MS. J. Anal. At. Spectrom. 22(2): 122-130

Miliszkiewicz N, Walas S, Tobiasz A (2015) Current approaches to calibration of LA-ICP-MS analysis. J. Anal. At. Spectrom. 30(2): 327-338

Norman MD, Pearson NJ, Sharma A, Griffin WL (1996) Quantitative analysis of trace elements in geological materials by laser ablation ICPMS: Instrumental operating conditions and calibration values of NIST glasses. Geostand. Newsl. 20(2): 247-261

Paton C, Hellstrom J, Paul B, Woodhead J, Hergt J (2011) Iolite: Freeware for the visualisation and processing of mass spectrometric data. J. Anal. At. Spectrom. 26(12): $2508-2518$

Paton C, Woodhead JD, Hellstrom JC, Hergt JM, Greig A, Maas R (2010) Improved laser ablation U-Pb zircon geochronology through robust downhole fractionation correction. Geochemistry, Geophysics, Geosystems. 11(3): 1-36

Russo RE, Mao X, Gonzalez JJ, Zorba V, Yoo J (2013) Laser Ablation in Analytical Chemistry. Analytical Chemistry. 85(13): 6162-6177 
Sylvester PJ (2008) Matrix effects in laser ablation ICP-MS. Laser Ablation ICP-MS in the Earth Sciences: Current Practices and Outstanding Issues (P. Sylvester, ed.). Mineralogical Association of Canada. Short Course Series. 40: 67-78

Tang M, Arevalo Jr R, Goreva Y, McDonough WF (2015) Elemental fractionation during condensation of plasma plumes generated by laser ablation: a ToF-SIMS study of condensate blankets. J. Anal. At. Spectrom. 30(11): 2316-2322

Wälle M, Koch J, Günther D (2008) Analysis of brass and silicate glass by femtosecond laser ablation inductively coupled plasma mass spectrometry using liquid standard calibration. J. Anal. At. Spectrom. 23(9): 1285

Wu S, Wang Y, Xu C, Yuan J (2016) Elemental Fractionation Studies of $193 \mathrm{~nm}$ ArF Excimer Laser Ablation System at High Spatial Resolution Mode. Chinese Journal of Analytical Chemistry. 44(7): 1035-1041

Wu S, Wang Y, Zhan X (2015) Research progress on reference materials for in situ elemental analysis by laser ablation-inductively coupled plasma-mass spectrometry. Rock and Mineral Analysis. 34(5): 503-511

Wu S, Wang Y, Zhan X, Kronz A, Simon K, Xu C, Tian H (2016) Study on the Elemental Fractionation Effect of CGSG Reference Materials and the Related Within-Unit Homogeneity of Major and Trace Elements. Rock and Mineral Analysis. 35(6): 612-620

Yuan J, Zhan X, Hu M, Zhao L, Sun D (2015) Characterization of Matrix Effects in Microanalysis of Sulfide Minerals by Laser Ablation-Inductively Coupled Plasma-Mass Spectrometry Based on An Element Pair Method. Spectroscopy and Spectral Analysis. 35(2): $512-518$ 
Yuan J, Zhan X, Sun D, Zhao L, Fan C, Kuai L, Hu M (2011) Investigationon Matrix Effects in Silicate Minerals by Laser Ablation-Inductively Coupled Plasma-Mass Spectrometry. Chinese Journal of Analytical Chemistry. 39(10): 1582-1588

Zack T, Hogmalm KJ (2016) Laser ablation Rb/Sr dating by online chemical separation of $\mathrm{Rb}$ and $\mathrm{Sr}$ in an oxygen-filled reaction cell. Chem. Geol. 437: 120-133

Zhang C, Hu Z, Zhang W, Liu Y, Zong K, Li M, Chen H, Hu S (2016) A green and fast laser fusion technique for bulk silicate rock analysis by laser ablation ICP-MS. Analytical Chemistry. 88(20): 10088-10094 


\section{Chapter 3 Further improvement the capability of LA-ICP-MS:}

\section{From sample preparation techniques to plasma enhancement}

Chapter 3.1 Comparison of Ultrafine Powder Pellet and Flux-free

Fusion Glass for Bulk Analysis of Granitic Rock Samples by Laser Ablation-Inductively Coupled Plasma-Mass Spectrometry

Shitou Wu*, Volker Karius, Burkhard C Schmidt, Klaus Simon and Gerhard Wörner

Geowissenschaftliches Zentrum, Göttingen Universität, Göttingen 37077, Germany

Corresponding author e-mail: wushitou111@hotmail.com

Fax: +49 $55139-23982$ 
Abstract: A comparison of sample preparation methods including ultrafine powder pellet and flux-free fusion glass for LA-ICP-MS analysis of granitic rock samples was carried out. The ultrafine powder particles are characterized as $\mathrm{d}_{50}=1.2 \mu \mathrm{m}, \mathrm{d}_{90}=5.5 \mu \mathrm{m}$. Our results illustrate that the agate abrasion was around 2-3\% relative to the mass of the original rock powders, however, contaminations with elements other than $\mathrm{SiO}_{2}$ from agate abrasion are negligible due to its chemicals are two magnitudes lower relative to original powders. A flux-free fusion protocol with a melting sequence of fusion, grinding and re-fusion was proposed for producing granitic glasses. The results demonstrate that the prerequisite for obtaining homogenous granitic glasses is the grinding procedure. Depletion of highly volatile elements (e.g. $\mathrm{Pb}$ ) was observed during fusion. Laser ablation rates are specified to the individual matrix. Analytical precisions of fusion glasses are comparable to MPI-DING glasses, while the precisions of powder pellets are slightly worse, which may be ascribed to the existence of remaining larger crystal fragments. Data accuracy demonstrates that preparation methods of ultrafine powder pellet and fusion glass are practical for LA-ICP-MS bulk analysis of granitic samples, which otherwise may suffer from incomplete dissolution in solution ICP-MS measurements.

Keywords: LA-ICP-MS; Granitic silicate; Ultrafine powder pellet; Flux-free fusion; Bulk analysis; Wet milling 


\subsubsection{Introduction}

Granitic rocks are a ubiquitous component of continental crust, and knowing their trace element signatures is essential in understanding their origins and evolution (Frost et al. 2001, Pearce et al. 1984, Whalen et al. 1987). In the past decades, several analytical techniques have been established for bulk analysis of granitic samples; most importantly are X-ray fluorescence (XRF) and inductively coupled plasma-mass spectrometry (ICP-MS). The relative high detection limit $(>10 \mu \mathrm{g} / \mathrm{g})$ of XRF makes it unsuitable for precise analysis of many trace elements in granites (Johnson et al. 1999, Peters and Pettke 2017). ICP-MS with acid/bomb digestion and subsequent analysis in solution, however, has deficiencies related to incomplete dissolution of accessory minerals (e.g. zircon, chromite) (Yu et al. 2001), the instability/adsorption of high valence trace elements (e.g. Ta) in acid solutions (Eggins 2003, Yu et al. 2000) as well as hazardous procedures involving the usage of strong acids (Yu et al. 2003, Zhang et al. 2016).

Laser ablation-inductively coupled plasma-mass spectrometry (LA-ICP-MS) provides advantages including high sensitivity, direct solid sampling and reduced laboratory work (Koch and Günther 2011, Liu et al. 2013, Russo et al. 2002, Sylvester and Jackson 2016) that could eliminate the deficiencies encountered with XRF and ICP-MS techniques. However, LA-ICP-MS is a microsampling technique, thus it requires the preparation of homogeneous and mechanical resistant targets to achieve controllable and representative sampling by laser ablation. Commonly preparation methods for LA-ICP-MS bulk analysis include the use of powder pellet (with or without binder) (Garbe-Schönberg and Müller 2014, Gray 1985, Jarvis and Williams 1993, Klemm and Bombach 2001, Mukherjee et al. 2014, Peters and Pettke 2017, Tabersky et al. 2014) and fusion glass (with or without flux) (Eggins 2003, Fedorowich et al. 1993, He et al. 2016, Park et al. 2016, Stoll et al. 2008, Sylvester 2001, Tamura et al. 2015, Yu et al. 2003, Zhang et al. 2016). Compared to fusion glasses, powder pellets of standard rock powders $(<74 \mu \mathrm{m})$ are inhomogeneous due to large grain size crystals (up to $74 \mu \mathrm{m}$, commonly milling procedure), and show problematical ablation behaviors because of the poor cohesion (Stoll et al. 2008, Zhang et al. 2016). 
Powder pellets are usually used for XRF analysis and later have been tested as the preparation method for LA-ICP-MS bulk analysis (Gray 1985). The introduction of a binder (polyvinyl alcohol, graphite, cellulose etc.) is necessary to enhance mechanical resistance for the powder pellets. However, a binder not only dilutes the trace element abundance but also introduces potential contaminations. Recently, a study by Garbe-Schönberg and Müller (2014) demonstrated that ultrafine powder pellets (produced with a wet-milling technique) without any binders had the excellent cohesive ability, and allowed the similar analytical precision compared to fusion glass. This wet-milling technique combined with a binder (microcrystalline cellulose) and subsequent analysis by LA-ICP-MS was evaluated and successfully used for analyzing a suite of geological materials (Peters and Pettke 2017). The ultrafine powder pellet technique thus provides the feasibility of bulk analysis of granitic samples using LA-ICP-MS. However, the wet-milling parameters (including ball-to-powder ratio, water-to-powder ratio, milling time) highly influence the milling efficiency and thus affect the quality of powder pellets and analytical results. In addition, the wet-milling protocol is specified to the sample matrix/type (Garbe-Schönberg and Müller 2014), thus further optimization of wet-milling protocol for granites needs to be developed prior to its application in LA-ICP-MS bulk analysis.

The fusion glass technique has been widely accepted as an alternative preparation method for LA-ICP-MS bulk analysis (Eggins 2003, Fedorowich et al. 1993). The addition of a properly chosen flux materials (e.g., lithium borate) could significantly lower the temperature required for completely dissolving silicate powders (Eggins 2003, Sylvester 2001, Yu et al. 2003). However, the flux introduces chemicals dilution and inevitable contaminations, and thus hampers the routine applications for LA-ICP-MS (Stoll et al. 2008). Flux-free fusion techniques by using tungsten strip (Fedorowich et al. 1993), iridium strip (Norman et al. 1996, Stoll et al. 2008), boron nitride vessel (Zhu et al. 2013), molybdenum strip (He et al. 2016), platinum capsule (Kurosawa et al. 2006) and infrared laser (Zhang et al. 2016) were developed to produce glasses from whole-rock powders. However, observations from these techniques illustrate that homogeneous glasses are easily produced for low silicate 
fine-grained rocks, but not for $\mathrm{SiO} 2$-rich coarse-grained rocks (granitic rocks). To produce homogeneous glasses from granitic samples usually needs a high temperature (>1500 oC) and long melting time (>30 min) to completely dissolve refractory minerals (e.g. Zircon) and homogeneously disperse chemical ingredients in the high viscosity melts (He et al. 2016). In this paper, two approaches are developed: (1) an optimized wet-milling protocol for granites with particle sizes characterization via laser particle size analyzer and electron microprobe. (2) A flux-free fusion protocol for producing homogeneous granitic glasses. The glass homogeneity and the depletion of volatile elements were evaluated by LA-ICP-MS analyses. Laser ablation rates of ultrafine powder pellets and fusion glasses were then compared. By using a series of granitic powder reference materials (GSP-2, G-2, JG-2, JG-3, GBW07103, and GBW07111) that are well characterized in previous studies, we compared the feasibility of these two preparation methods for LA-ICP-MS analysis of granitic samples.

\subsubsection{Materials and Methods}

\subsubsection{Granitic silicates}

A suite of six coarse-grained intrusive granitic rock reference materials (GSP-2, G-2, JG-2, JG-3, GBW 07103 and GBW 07111) with $\mathrm{SiO} 2$ contents from 59.68 to 76.83 wt\% was investigated. Detailed information of the investigated materials is summarized in Table 1. Reference values of GSP-2 were compiled based on data from Wilson (1998), Raczek et al. (2001) and Pretorius et al. (2006). Reference values of G-2 were obtained from Jochum et al. (2016). Reference values of JG-2 and JG-3 were originally from Imai et al. (1995). Reference values of GBW 07103 and GBW 07111 were collected from National Research Center for Certified Reference Materials, China.

\subsubsection{Preparation methods}

\subsection{Ultrafine powder pellets}

A high-performance planetary ball mill (Fritsch Pulverisette 7 premium line, FRITSCH $\mathrm{GmbH}$ ) configured with $20 \mathrm{ml}$ agate vials and $5 \mathrm{~mm}$ agate balls were used to produce ultrafine powders. Eighty agate balls (ca. $15.9 \mathrm{~g}$ ) and $2.78 \mathrm{~g}$ original powders $(<74 \mu \mathrm{m})$ were 
Chapter 3.1 Comparison of Ultrafine Powder Pellet and Flux-free Fusion Glass for Bulk Analysis of Granitic Rock Samples by Laser Ablation-Inductively Coupled Plasma-Mass Spectrometry

added into the vial. Afterward, $2.5 \mathrm{~mL}$ of ultrapure water was pipetted into the vial. All agate balls were weighted before and after the milling process in order to calculate the loss of agate due to abrasion. The wet-milling procedure comprised of fifteen consecutive milling cycles at the powder of $900 \mathrm{rpm}$. Each cycle incorporated a three-minute milling interval followed by a twenty-second pause for cooling. The rotation direction was reversed between each interval. After completion, the suspensions were transferred into a clean beaker and then moved into a freeze dryer (Alpha 1- 4 LD, Martin Christ $\mathrm{GmbH}$ ). The freezing-dried powders were re-homogenized for two minutes by using a homogenizer (Fritsch Pulverisette 23, FRITSCH $\mathrm{GmbH})$. To produce powder pellets, we used two-inch diameter custom-made cellulose plates in which we formed fifteen holes of $5 \mathrm{~mm}$ diameter and $2 \mathrm{~mm}$ depth as casts to hold the powder using a custom-designed steel plate. Then, approximately $25 \mathrm{mg}$ powders were filled into the holes and pelleted with the pressure of $20 \mathrm{t}$ for two minutes. A HERZOG HTP 40 presser was used for the pellet pressing. The pressed powder pellets are shown in Fig.1a.

Table 1 Reference materials investigated in this study.

\begin{tabular}{cccccc}
\hline Samples & Classification & Origins & Supplier & Category & Source of reference values \\
\hline GSP-2 & Granodiorite & Silver Plume,Colorado, USA & USGS & CRM & Wilson 1998, Raczek et al.2001, Pretorius et al. 2006 \\
G-2 & Granite & Sullivan quarry, Bradford, Rhode Island & UGSG & CRM & Jochum et al. 2016 \\
JG-2 & Granite & Cretaceous, Hirukawa-mura, Gifu Prefecture, Japan & GSJ & RM & Imai et al. 1995 \\
JG-3 & Granodiorite & Mitoya-cho, Shimane Prefecture, Japan & GSJ & RM & Collected from NRCC \\
GBW07103 & Granite & Qianlishan, Chenzhou, Hunan, China & NRCC & CRM & Collected from NRCC \\
\hline GBW07111 & Granodiorite & Zhoukou, Fangshan, Beijing, China & NRCC & CRM & C
\end{tabular}

USGS: United States Geological Survey, USGS; GSJ: Geological Survey of Japan, Japan; NRCC: National Research Center for Certified Reference Materials, China; CRM: Certified Reference Material; RM: Reference Material.

The grain size analysis was done with a laser particle size analyzer (LS 13320 Beckman\&Coulter). All samples were treated with ultrasonic dispersion for the $30 \mathrm{~s}(0.34 \mathrm{~kJ})$ before measurement. The measurement was conducted with PIDS-data using a Quartz model with R.I. 0.556/0.1., 3 runs, the 60s each run. The elemental mapping and SEM images were 
Chapter 3.1 Comparison of Ultrafine Powder Pellet and Flux-free Fusion Glass for Bulk Analysis of Granitic Rock Samples by Laser Ablation-Inductively Coupled Plasma-Mass Spectrometry

acquired with an EPMA (JELJXA-8900, Japan Electron Optics Laboratory Company, Japan).

The acceleration voltage was $15 \mathrm{kV}$, beam current was $15 \mathrm{nA}$ and beam diameter was $1 \mu \mathrm{m}$.

\subsection{Flux-free fusion glasses}

The flux-free fusion glasses were produced from granitic silicates using a high-temperature furnace (LTH 4 R, Nabertherm $\mathrm{GmbH}$ ) at atmospheric environment. Approximately $2 \mathrm{~g}$ original rock powders were weighed into a platinum crucible $(30 \mathrm{ml})$ with lid and then transferred to the furnace for one-hour fusion at $1600{ }^{\circ} \mathrm{C}$. Afterward, the silicate melts were quenched to glasses by dipping the bottom of the crucible into cold water. During this procedure, no water got in direct contact with the melts or glasses. The glasses were carefully removed from the crucible and then ground in an automatic agate mortar (RE 100, Retsch $\mathrm{GmbH})$ for fifteen minutes. The re-ground powders were transferred into the platinum crucible and placed into the furnace for a second one-hour fusion at $1600{ }^{\circ} \mathrm{C}$. One more grinding step was applied for JG-2 due to the high $\mathrm{SiO}_{2}$ content, namely, $1 \mathrm{~h}$ melting, $15 \mathrm{~min}$ grinding, $1 \mathrm{~h}$ melting, $15 \mathrm{~min}$ grinding, $2 \mathrm{~h}$ melting. The re-melted glasses were carefully removed from the crucible, and then mounted in epoxy resin (one inch) and polished to a flat surface. The fusion glasses embedded in epoxy resin are shown in Fig.1b.
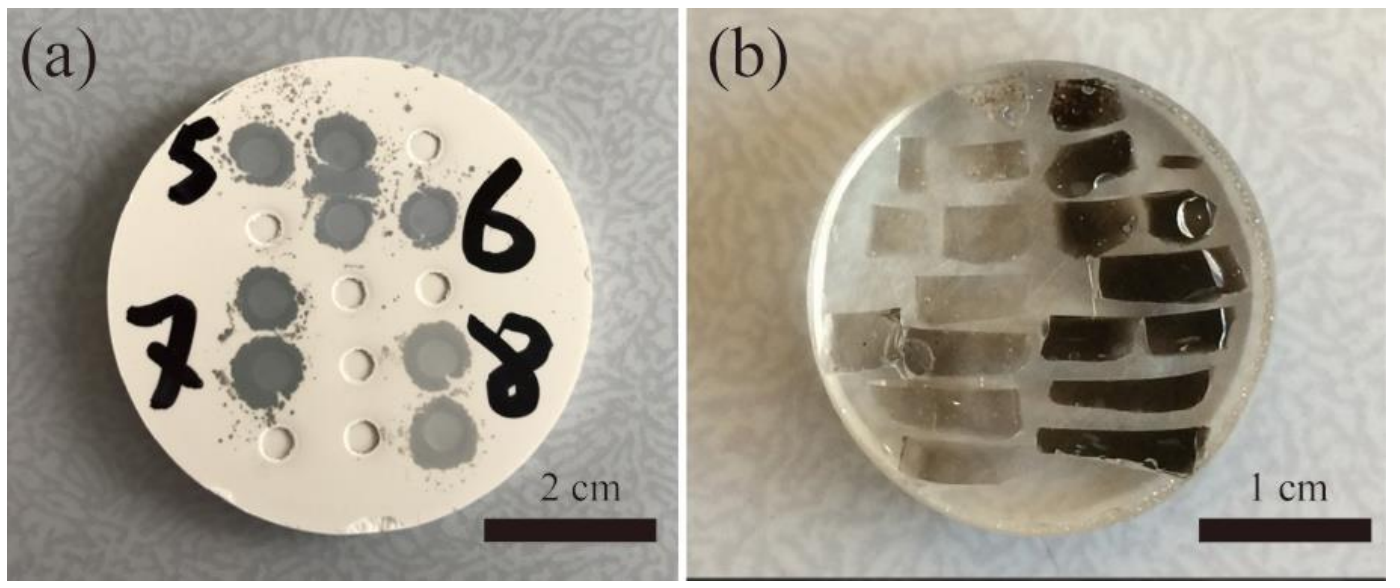

Fig.1 Photography of (a) ultrafine powder pellets tableted in a cellulose holder (two inch), (b) flux-free fusion glasses mounted in epoxy resin (one inch). Four different samples are in the pellet (a). Two different samples, each with ten glass pieces, are in epoxy resin (b). 


\subsubsection{LA-ICP-MS and data acquisition}

A $193 \mathrm{~nm}$ ArF excimer laser ablation system (RESOlution M-50, ASI, Australia) coupled to an Element 2 sector field ICP-MS (ThermoScientific, USA) was used for measurements. The powder pellets, fusion glasses, and external reference materials were loaded into the Laurin Technic S-155 laser ablation cell (see Appendix S1). Helium was employed as a carrier gas for signal enhancement (Eggins et al. 1998). A 'squid' smooth device was used for improving signal precision (Müller et al. 2009). The instrument conditions were optimized by ablating NIST 612 in raster mode to achieve the highest ${ }^{139} \mathrm{La}$ intensity while keeping the U/Th around 1 , oxide $(\mathrm{ThO} / \mathrm{Th})$ and secondary ion production $\left({ }^{2+} \mathrm{Ca} /{ }^{+} \mathrm{Ca}\right)$ were lower than $0.5 \%$. The operating conditions of LA-ICP-MS are summarized in Table 2.

The LA-ICP-MS transient data were collected at discrete spot mode with a spot size of 50 and $90 \mu \mathrm{m}$ for fusion glasses and powder pellets, respectively. A larger spot size was used for powder pellet to account for the potential powder heterogeneity. Generally, eight single analyses were performed for each sample (in two individual pellets or glass fragments). Each individual analysis incorporated a 20-second background acquisition (gas blank) followed by a 35-second ablation data acquisition. A pre-ablation procedure (two laser pulses) was performed to avoid the potential influence caused by aerosol deposits and other form of surface contaminations. The Iolite 3.4 software package was used for the data reduction using the 'laser log file' for automatically setting the background and integration intervals for each analysis (Paton et al. 2011). To reduce the effect of potentially unstable signals, the initial five and last three seconds of each sample acquisition were discarded from data integration. The quantification was processed using $\mathrm{Al}$ as internal standard (using the reference values) and NIST610, GSD-1G, and StHs6/80-G as external reference materials. The bulk normalization as $100 \% \mathrm{~m} / \mathrm{m}$ strategy was also used for fusion glass. The reference values of NIST610, GSD-1G, and StHs6/80-G were collected from the literature (Hu et al. 2008, Jochum et al. 2006, Jochum et al. 2011) as well as the GeoReM database (http://georem.m pch-mainz.gwdg.de/). 
Table 2 Operating conditions of LA-ICP-MS system.

Laser ablation system

Laser type

ArF Excimer

Wavelength

$193 \mathrm{~nm}$

Pulse time

$20 \mathrm{~ns}$

Energy density

around $5.0 \mathrm{~J} / \mathrm{cm}^{2}$

Repetition rate

$5 \mathrm{~Hz}$

Ablation cell

Laurin Technic S-155

Spot size

$50,90 \mu \mathrm{m}$

Ablation gas flow (He)

$0.65 \mathrm{~L} / \mathrm{min}$

Ablation time

$35 \mathrm{~s}$

ICP-MS

ICP-MS

Element 2

RF Power

$1400 \mathrm{~W}$

Guard electrode

Floated and grounded (Pt)

Sample cone

Ni 54605

Skimmer cone

Ni $543540.8 \mathrm{H}$

Coolant gas flow (Ar)

$15.00 \mathrm{~L} / \mathrm{min}$

Auxiliary gas flow (Ar)

$1.00 \mathrm{~L} / \mathrm{min}$

Carrier gas flow (Ar)

$0.95 \mathrm{~L} / \mathrm{min}$

Scan mode

E-scan

Segment duration

$10 \mathrm{~ms}$

Detector

Dual (counting and analog)

Resolution (M/DM)

Low (around 300)

Time pre pass

$1 \mathrm{~s}$

\subsubsection{Data compilation}

LA-ICP-MS data were compiled from the results obtained from NIST610, StHs6/80-G, and GSD-1G as external reference materials. Some elements occur in extremely low contents (Ti, Fe, $\mathrm{Mn}, \mathrm{Mg}, \mathrm{K}$, and $\mathrm{P}$ in NIST610, Be, Mo and Sb in StHs6/80-G) or are under/overestimated certified values (Cr, Ni in StHs6/80-G and $\mathrm{Sn}, \mathrm{Sb}$ in GSD-1G) in reference materials. The data calibrated with these elements are excluded from the compilation. The $\mathrm{SiO}_{2}$ 
concentrations obtained from ultrafine powder pellets are not given due to the contamination by the agate abrasion. $\mathrm{Ni}$ and $\mathrm{Pb}$ suffered contamination or depletion during fusion procedure, thus these two elements are not given in the fusion glass dataset. Results calibrated based on bulk normalization as $100 \% \mathrm{~m} / \mathrm{m}$ strategy for the fusion glasses are nearly identical to internal standardization (data not shown here). The analytical results of both preparation methods are shown in Table 3, 4 and 5. Elements marked with "*”" mean that the reference values are given as uncertified values for information.

\subsubsection{Results and discussion}

\subsubsection{Optimization of wet-milling parameters}

The wet-milling parameters (including ball-to-powder ratio, water-to-powder ratio, milling time) are critical for the milling efficiency, and can significantly influence the quality of LA-ICP-MS analyses. For the optimization experiments, a pulverized granite in-house reference material (G1RF) was used, which contains a high abundance of zircon and biotite. Different from previous studies by Garbe-Schönberg and Müller (2014) and Peters and Pettke (2017), a smaller volume vial $(20 \mathrm{ml})$ and slightly smaller agate balls $(5 \mathrm{~mm}$ in diameter) were used for the wet-milling procedure.

The mill manufacturer recommends that eighty agate balls $(5 \mathrm{~mm}$ in diameter) should be filled into the $20 \mathrm{ml}$ vials and the powder volume (grain volume) should be kept in a range from $1 \mathrm{ml}$ to $9 \mathrm{ml}$. Here, $1.05 \mathrm{ml}$ grain volume samples (assumed density $2.65 \mathrm{~g} / \mathrm{cm}^{3}$ ) were used for the wet-milling. The addition of water should maintain all particles in a suspension and significantly improve the milling efficiency (Garbe-Schönberg and Müller 2014). Too little water leads to enhanced stickiness of the suspension, while too much water will reduce the collision energy and therefore reduces the milling efficiency. For the granites used in this study, a water to powder ratio of 2.5 (volume/volume) seemed to be optimal and was kept for all subsequent millings. 
Chapter 3.1 Comparison of Ultrafine Powder Pellet and Flux-free Fusion Glass for Bulk Analysis of Granitic Rock Samples by Laser Ablation-Inductively Coupled Plasma-Mass Spectrometry

Table 3 Analytical results of GSP-2 and G-2 obtained from ultrafine powder pellet and flux-free fusion glass techniques.

The data are complied with NIST610, StHs6/80-G and GSD-1G as external reference materials.

\begin{tabular}{|c|c|c|c|c|c|c|c|c|c|c|c|c|c|c|c|c|}
\hline \multirow[b]{3}{*}{ Elements } & \multicolumn{8}{|c|}{ GSP-2 } & \multicolumn{8}{|c|}{ G-2 } \\
\hline & \multicolumn{2}{|c|}{ Reference value } & \multicolumn{3}{|c|}{ Ultrafine powder pellet } & \multicolumn{3}{|c|}{ Flux-free fusion glass } & \multicolumn{2}{|c|}{ Reference value } & \multicolumn{3}{|c|}{ Ultrafine powder pellet } & \multicolumn{3}{|c|}{ Flux-free fusion glass } \\
\hline & Value & Uncertainty & Average & $1 \mathrm{~s}$ & $\mathrm{RD}(\%)$ & Average & $1 \mathrm{~s}$ & $\mathrm{RD}(\%)$ & Value & Uncertainty & Average & $1 \mathrm{~s}$ & $\mathrm{RD}(\%)$ & Average & $1 \mathrm{~s}$ & $\mathrm{RD}(\%)$ \\
\hline $\mathrm{SiO}_{2}$ & 66.6 & 0.8 & - & - & - & 67.7 & 0.346 & 1.70 & 68.7 & 0.47 & - & - & - & 69.4 & 0.326 & 0.93 \\
\hline $\mathrm{TiO}_{2}$ & 0.66 & 0.02 & 0.662 & 0.0253 & 0.31 & 0.684 & 0.0275 & 3.61 & 0.48 & 0.0089 & 0.491 & 0.0211 & 2.33 & 0.497 & 0.0201 & 3.59 \\
\hline $\mathrm{Al}_{2} \mathrm{O}_{3}$ & 14.9 & 0.2 & 14.9 & 0.432 & 0.00 & 14.9 & 0.326 & 0.00 & 15.3 & 0.17 & 15.3 & 0.385 & 0.00 & 15.3 & 0.381 & 0.00 \\
\hline $\mathrm{FeO}(\mathrm{t})$ & 4.41 & 0.144 & 4.49 & 0.285 & 1.90 & 4.59 & 0.0677 & 4.15 & 2.38 & 0.0432 & 2.56 & 0.229 & 7.46 & 2.49 & 0.0434 & 4.55 \\
\hline $\mathrm{MnO}$ & 0.0413 & 0.0026 & 0.0427 & 0.0017 & 3.24 & 0.0432 & 0.0006 & 4.51 & 0.0306 & 0.0008 & 0.0347 & 0.0014 & 13.30 & 0.034 & 0.0004 & 11.10 \\
\hline $\mathrm{MgO}$ & 0.96 & 0.03 & 0.941 & 0.0282 & -2.01 & 0.955 & 0.0089 & -0.56 & 0.754 & 0.029 & 0.757 & 0.0273 & 0.44 & 0.731 & 0.0111 & -3.03 \\
\hline $\mathrm{CaO}$ & 2.1 & 0.06 & 2.17 & 0.0896 & 3.30 & 2.21 & 0.0832 & 5.00 & 1.91 & 0.037 & 2.05 & 0.0665 & 7.12 & 2.09 & 0.0929 & 9.31 \\
\hline $\mathrm{Na}_{2} \mathrm{O}$ & 2.78 & 0.09 & 2.81 & 0.0832 & 0.96 & 2.76 & 0.0336 & -0.79 & 4.05 & 0.059 & 4.09 & 0.105 & 1.11 & 3.99 & 0.0471 & -1.35 \\
\hline $\mathrm{K}_{2} \mathrm{O}$ & 5.38 & 0.14 & 5.46 & 0.191 & 1.48 & 5.62 & 0.127 & 4.40 & 4.5 & 0.061 & 4.61 & 0.0457 & 2.44 & 4.63 & 0.0966 & 2.88 \\
\hline $\mathrm{P}_{2} \mathrm{O}_{5}$ & 0.29 & 0.02 & 0.291 & 0.0255 & 0.20 & 0.291 & 0.0292 & 0.27 & 0.129 & 0.022 & 0.141 & 0.013 & 9.20 & 0.127 & 0.0125 & -1.92 \\
\hline $\mathrm{Li}^{*}$ & 36 & 1 & 37 & 1.95 & 2.91 & 37.5 & 1.52 & 4.22 & 33.6 & 2.9 & 34.7 & 1.42 & 3.22 & 35.7 & 2.31 & 6.23 \\
\hline $\mathrm{Be}^{*}$ & 1.5 & 0.2 & 1.92 & 0.448 & 28.20 & 1.45 & 1.07 & -3.57 & 2.49 & 0.081 & 2.86 & 0.649 & 15.10 & 3.01 & 1.16 & 21.00 \\
\hline $\mathrm{Sc}$ & 6.3 & 0.7 & 6.91 & 2.67 & 9.70 & 8.32 & 0.62 & 32.10 & 3.66 & 0.12 & 4.83 & 0.307 & 32.10 & 5.11 & 0.585 & 39.70 \\
\hline $\mathrm{V}$ & 52 & 4 & 57.3 & 3.97 & 10.10 & 56.3 & 2.83 & 8.18 & 35.1 & 0.81 & 38.9 & 3.42 & 10.80 & 37 & 1.87 & 5.43 \\
\hline $\mathrm{Cr}$ & 20 & 6 & 20 & 7.61 & 0.09 & 17.5 & 1.53 & -12.42 & 7.88 & 0.38 & 8.48 & 0.895 & 7.62 & 6.23 & 0.752 & -20.89 \\
\hline Co & 7.3 & 0.8 & 7.1 & 0.317 & -2.75 & 7.12 & 0.247 & -2.52 & 4.48 & 0.098 & 4.58 & 0.343 & 2.18 & 4.41 & 0.227 & -1.52 \\
\hline $\mathrm{Ni}$ & 17 & 2 & 14.8 & 1.4 & -12.68 & - & - & - & 3.46 & 0.57 & 2.29 & 0.459 & -33.84 & - & - & - \\
\hline $\mathrm{Cu}$ & 43 & 4 & 38 & 1.92 & -11.70 & 22.6 & 1.2 & -47.51 & 11 & 0.65 & 11.8 & 9.96 & 7.37 & 12.5 & 0.907 & 14.20 \\
\hline $\mathrm{Zn}$ & 120 & 10 & 145 & 5.44 & 20.90 & 123 & 9.65 & 2.61 & 83.5 & 1 & 111 & 5.67 & 33.50 & 93.5 & 5.23 & 12.00 \\
\hline $\mathrm{Ga}$ & 22 & 2 & 24.2 & 0.843 & 9.85 & 25.6 & 1.86 & 16.20 & 23.3 & 0.52 & 23.2 & 0.939 & -0.56 & 23 & 1.64 & -1.50 \\
\hline $\mathrm{Rb}$ & 245 & 7 & 259 & 9.48 & 5.62 & 259 & 8.9 & 5.53 & 169 & 2.9 & 182 & 7.93 & 8.31 & 178 & 6.33 & 5.65 \\
\hline $\mathrm{Sr}$ & 240 & 10 & 231 & 5.51 & -3.74 & 237 & 5.49 & -1.41 & 475 & 6.1 & 464 & 10.6 & -2.24 & 475 & 12.5 & 0.10 \\
\hline $\mathrm{Y}$ & 28 & 2 & 24.2 & 1.55 & -13.57 & 25.2 & 1.2 & -9.87 & 9.88 & 0.17 & 9.09 & 0.425 & -8.04 & 9.46 & 0.606 & -4.25 \\
\hline $\mathrm{Zr}$ & 550 & 30 & 523 & 31.5 & -4.97 & 564 & 24.7 & 2.59 & 319 & 7.8 & 305 & 65.6 & -4.47 & 324 & 14.4 & 1.55 \\
\hline $\mathrm{Nb}$ & 27 & 2 & 26.2 & 1.49 & -2.96 & 27.4 & 1.67 & 1.36 & 12 & 0.24 & 12.5 & 0.614 & 3.94 & 12.5 & 0.657 & 4.05 \\
\hline Mo* & 2.1 & 0.6 & 1.79 & 1.01 & -14.95 & 2.48 & 0.19 & 18.10 & 0.38 & 0.14 & 0.18 & 0.0432 & -52.73 & 0.408 & 0.105 & 7.43 \\
\hline $\mathrm{Cs}^{*}$ & 1.2 & 0.1 & 1.28 & 0.0772 & 6.61 & 1.3 & 0.139 & 8.18 & 1.36 & 0.021 & 1.53 & 0.0811 & 13.10 & 1.5 & 0.139 & 10.40 \\
\hline $\mathrm{Ba}$ & 1340 & 44 & 1312 & 23.3 & -2.08 & 1321 & 20.2 & -1.44 & 1860 & 17 & 1831 & 41.2 & -1.55 & 1849 & 28.5 & -0.57 \\
\hline $\mathrm{La}$ & 180 & 12 & 177 & 13.2 & -1.58 & 184 & 6.35 & 2.46 & 88.4 & 1.4 & 86.3 & 4.74 & -2.36 & 87.8 & 3.2 & -0.71 \\
\hline $\mathrm{Ce}$ & 410 & 30 & 430 & 30.2 & 4.91 & 439 & 13.5 & 7.13 & 161 & 2.3 & 161 & 7.05 & -0.04 & 161 & 5.07 & -0.32 \\
\hline $\operatorname{Pr}^{*}$ & 51 & 5 & 52.7 & 4.09 & 3.34 & 54.2 & 1.52 & 6.20 & 16.9 & 0.3 & 15.8 & 0.772 & -6.22 & 15.9 & 0.422 & -5.86 \\
\hline $\mathrm{Nd}$ & 200 & 12 & 196 & 15.4 & -2.08 & 205 & 5.77 & 2.73 & 53.8 & 0.67 & 51.7 & 2.59 & -3.86 & 53.1 & 2.17 & -1.24 \\
\hline $\mathrm{Sm}$ & 27 & 1 & 24.9 & 1.97 & -7.67 & 26.6 & 1.73 & -1.36 & 7.19 & 0.1 & 6.93 & 0.283 & -3.60 & 7.42 & 0.595 & 3.14 \\
\hline $\mathrm{Eu}$ & 2.3 & 0.1 & 2.19 & 0.166 & -4.81 & 2.27 & 0.164 & -1.20 & 1.41 & 0.028 & 1.31 & 0.0619 & -7.70 & 1.28 & 0.129 & -9.71 \\
\hline $\mathrm{Gd}^{*}$ & 12 & 2 & 11.6 & 0.646 & -2.93 & 12.4 & 1 & 3.38 & 4.23 & 0.16 & 3.79 & 0.302 & -10.32 & 3.81 & 0.308 & -9.93 \\
\hline $\mathrm{Tb \#}$ & 1.3 & 0.0501 & 1.15 & 0.0708 & -11.16 & 1.22 & 0.0685 & -6.07 & 0.495 & 0.019 & 0.399 & 0.0225 & -19.47 & 0.424 & 0.0386 & -14.35 \\
\hline Dy* & 5.9 & 0.1 & 5.43 & 0.471 & -7.96 & 5.65 & 0.279 & -4.27 & 2.28 & 0.063 & 2.02 & 0.134 & -11.50 & 2.05 & 0.117 & -10.04 \\
\hline $\mathrm{Ho}^{*}$ & 1 & 0.1 & 0.881 & 0.0557 & -11.90 & 0.95 & 0.0794 & -4.98 & 0.373 & 0.0098 & 0.342 & 0.0296 & -8.19 & 0.363 & 0.0407 & -2.46 \\
\hline $\mathrm{Er}^{*}$ & 2.39 & 0.0299 & 2.19 & 0.206 & -8.30 & 2.23 & 0.215 & -6.89 & 0.927 & 0.021 & 0.855 & 0.0773 & -7.77 & 0.913 & 0.111 & -1.54 \\
\hline $\mathrm{Tm}^{*}$ & 0.29 & 0.02 & 0.28 & 0.0254 & -3.53 & 0.29 & 0.0361 & -0.03 & 0.123 & 0.0046 & 0.114 & 0.0166 & -7.60 & 0.111 & 0.0234 & -10.00 \\
\hline $\mathrm{Yb}$ & 1.6 & 0.2 & 1.55 & 0.168 & -3.12 & 1.65 & 0.192 & 3.43 & 0.722 & 0.02 & 0.692 & 0.0915 & -4.19 & 0.683 & 0.105 & -5.44 \\
\hline $\mathrm{Lu}^{*}$ & 0.23 & 0.03 & 0.222 & 0.0247 & -3.56 & 0.221 & 0.0321 & -3.82 & 0.102 & 0.0039 & 0.0998 & 0.0179 & -2.05 & 0.0907 & 0.0156 & -10.95 \\
\hline $\mathrm{Hf}^{*}$ & 14 & 1 & 13.9 & 0.767 & -1.06 & 14.5 & 0.541 & 3.86 & 7.78 & 0.22 & 7.42 & 1.54 & -4.60 & 7.75 & 0.552 & -0.35 \\
\hline Ta\# & 0.87 & 0.0413 & 0.835 & 0.0724 & -3.97 & 0.87 & 0.0867 & 0.05 & 0.834 & 0.031 & 0.786 & 0.0679 & -5.71 & 0.78 & 0.0579 & -6.42 \\
\hline $\mathrm{Pb}$ & 42 & 3 & 53.3 & 3.72 & 26.90 & - & - & - & 30 & 0.66 & 35.8 & 1.84 & 19.40 & - & - & - \\
\hline Th & 105 & 8 & 105 & 9.96 & -0.34 & 112 & 4.03 & 6.30 & 24.5 & 0.39 & 24.3 & 1.74 & -0.86 & 24.3 & 1.35 & -0.99 \\
\hline U & 2.4 & 0.19 & 2.41 & 0.127 & 0.28 & 2.45 & 0.144 & 2.10 & 1.96 & 0.067 & 1.48 & 0.117 & -24.68 & 1.99 & 0.117 & 1.56 \\
\hline
\end{tabular}


Chapter 3.1 Comparison of Ultrafine Powder Pellet and Flux-free Fusion Glass for Bulk Analysis of Granitic Rock Samples by Laser Ablation-Inductively Coupled Plasma-Mass Spectrometry

Table 4 Analytical results of GBW07103 and GBW07111 obtained from powder pellet and flux-free fusion glass techniques. The data are complied with NIST610, StHs6/80-G and GSD-1G as external reference materials.

\begin{tabular}{|c|c|c|c|c|c|c|c|c|c|c|c|c|c|c|c|c|}
\hline \multirow[b]{3}{*}{ Elements } & \multicolumn{8}{|c|}{ GBW07103 } & \multicolumn{8}{|c|}{ GBW07111 } \\
\hline & \multicolumn{2}{|c|}{ Reference value } & \multicolumn{3}{|c|}{ Ultrafine powder pellet } & \multicolumn{3}{|c|}{ Flux-free fusion glass } & \multicolumn{2}{|c|}{ Reference value } & \multicolumn{3}{|c|}{ Ultrafine powder pellet } & \multicolumn{3}{|c|}{ Flux-free fusion glass } \\
\hline & Value & Uncertainty & Average & $1 \mathrm{~s}$ & $\mathrm{RD}(\%)$ & Average & $1 \mathrm{~s}$ & $\mathrm{RD}(\%)$ & Value & Uncertainty & Average & $1 \mathrm{~s}$ & $\mathrm{RD}(\%)$ & Average & $1 \mathrm{~s}$ & $\mathrm{RD}(\%)$ \\
\hline $\mathrm{SiO}_{2}$ & 72.8 & 0.15 & - & - & - & 73.5 & 0.543 & 0.90 & 59.7 & 0.15 & - & - & - & 61.1 & 0.597 & 2.43 \\
\hline $\mathrm{TiO}_{2}$ & 0.287 & 0.0167 & 0.293 & 0.012 & 2.05 & 0.29 & 0.0141 & 1.06 & 0.77 & 0.04 & 0.739 & 0.0207 & -4.02 & 0.764 & 0.0259 & -0.78 \\
\hline $\mathrm{Al}_{2} \mathrm{O}_{3}$ & 13.4 & 0.11 & 13.4 & 0.374 & 0.00 & 13.4 & 0.344 & 0.00 & 16.6 & 0.14 & 16.6 & 0.412 & 0.00 & 16.6 & 0.331 & 0.00 \\
\hline $\mathrm{FeO}(\mathrm{t})$ & 1.93 & 0.072 & 2.04 & 0.175 & 5.92 & 2.03 & 0.139 & 5.17 & 5.46 & 0.17 & 5.7 & 0.188 & 4.46 & 5.77 & 0.0785 & 5.74 \\
\hline $\mathrm{MnO}$ & 0.0598 & 0.0023 & 0.0619 & 0.0016 & 3.54 & 0.0637 & 0.0027 & 6.60 & 0.094 & 0.0035 & 0.101 & 0.0048 & 7.20 & 0.101 & 0.0015 & 7.66 \\
\hline $\mathrm{MgO}$ & 0.42 & 0.05 & 0.398 & 0.011 & -5.21 & 0.407 & 0.0108 & -3.15 & 2.81 & 0.1 & 2.79 & 0.042 & -0.75 & 2.73 & 0.0475 & -2.91 \\
\hline $\mathrm{CaO}$ & 1.55 & 0.07 & 1.62 & 0.0608 & 4.36 & 1.98 & 0.33 & 27.70 & 4.72 & 0.12 & 4.95 & 0.14 & 4.78 & 5.01 & 0.14 & 6.11 \\
\hline $\mathrm{Na}_{2} \mathrm{O}$ & 3.13 & 0.09 & 3.14 & 0.141 & 0.33 & 2.95 & 0.0752 & -5.78 & 4.05 & 0.08 & 4.06 & 0.125 & 0.17 & 4.13 & 0.049 & 1.94 \\
\hline $\mathrm{K}_{2} \mathrm{O}$ & 5.01 & 0.1 & 5.17 & 0.214 & 3.16 & 5.11 & 0.304 & 2.00 & 3.5 & 0.08 & 3.59 & 0.0676 & 2.65 & 3.73 & 0.0707 & 6.58 \\
\hline $\mathrm{P}_{2} \mathrm{O}_{5}$ & 0.0928 & 0.0046 & 0.0894 & 0.0105 & -3.71 & 0.0817 & 0.0086 & -11.93 & 0.34 & 0.0069 & 0.349 & 0.0342 & 2.50 & 0.317 & 0.033 & -6.69 \\
\hline $\mathrm{Li}$ & 131 & 7 & 145 & 4.93 & 11.00 & 133 & 6.33 & 1.80 & 16.2 & 2.1 & 18 & 1.09 & 11.30 & 19.3 & 1.55 & 19.10 \\
\hline $\mathrm{Be}$ & 12.4 & 2.1 & 13.8 & 1.89 & 11.40 & 15 & 2.69 & 20.60 & 2.11 & 0.78 & 2.15 & 0.803 & 2.01 & 2.25 & 1.53 & 6.42 \\
\hline $\mathrm{Sc}$ & 6.1 & 0.6 & 7.19 & 0.501 & 17.90 & 7.5 & 0.583 & 22.90 & 10.3 & 1.1 & 11.9 & 0.758 & 15.70 & 12.4 & 0.968 & 20.50 \\
\hline $\mathrm{V}$ & 24 & 3 & 23.8 & 2.28 & -0.78 & 23 & 1.23 & -4.36 & 104 & 7 & 114 & 2.83 & 9.88 & 114 & 5.16 & 9.23 \\
\hline $\mathrm{Cr}$ & 3.6 & 1.1 & 2.39 & 0.511 & -33.71 & 2.82 & 1.23 & -21.65 & 37.6 & 3.4 & 41.6 & 5.37 & 10.50 & 35.1 & 3.92 & -6.66 \\
\hline Co & 3.4 & 1 & 2.75 & 0.217 & -19.02 & 3.45 & 0.307 & 1.35 & 15.6 & 1.2 & 16.2 & 0.501 & 3.76 & 15.9 & 0.498 & 2.17 \\
\hline $\mathrm{Ni}$ & 2.3 & 1.2 & 1.28 & 0.475 & -44.16 & - & - & - & 24.4 & 2.3 & 33.6 & 4.83 & 37.60 & - & - & - \\
\hline $\mathrm{Cu}$ & 3.2 & 1.3 & 1.67 & 0.7 & -47.69 & 4.05 & 0.696 & 26.60 & 8.8 & 1.5 & 8.9 & 0.739 & 1.16 & 8.84 & 0.673 & 0.43 \\
\hline $\mathrm{Zn}$ & 28 & 4 & 35.1 & 5.6 & 25.50 & 25.7 & 3.1 & -8.24 & 85.4 & 9.4 & 116 & 6.55 & 35.80 & 92.6 & 5.9 & 8.38 \\
\hline $\mathrm{Ga}$ & 19 & 2 & 17.9 & 1.28 & -5.76 & 19.5 & 1.42 & 2.67 & 20.8 & 2.4 & 21.3 & 0.905 & 2.31 & 22 & 1.81 & 5.86 \\
\hline $\mathrm{Rb}$ & 466 & 26 & 507 & 19.8 & 8.72 & 477 & 19.8 & 2.30 & 70.1 & 11 & 78.7 & 3.92 & 12.30 & 78.8 & 3.03 & 12.40 \\
\hline $\mathrm{Sr}$ & 106 & 9 & 104 & 2.39 & -1.71 & 107 & 4.33 & 0.76 & 1198 & 88 & 1227 & 31.6 & 2.41 & 1244 & 28.9 & 3.88 \\
\hline $\mathrm{Y}$ & 62 & 7 & 60.3 & 5.91 & -2.69 & 64.6 & 3.25 & 4.27 & 15.5 & 1.7 & 16.3 & 0.78 & 5.08 & 16.6 & 0.914 & 6.81 \\
\hline $\mathrm{Zr}$ & 167 & 14 & 148 & 25 & -11.25 & 163 & 7.72 & -2.40 & 224 & 29 & 211 & 19.5 & -5.82 & 235 & 8.3 & 4.72 \\
\hline $\mathrm{Nb}$ & 40 & 4 & 41.7 & 2.48 & 4.20 & 43.2 & 2.28 & 7.90 & 10.6 & 1.4 & 10.6 & 0.577 & -0.29 & 10.5 & 0.364 & -1.16 \\
\hline Mo & 3.5 & 0.3 & 4.94 & 5.4 & 41.10 & 3.73 & 0.639 & 6.53 & 0.47 & 0.4 & 0.509 & 0.0486 & 8.28 & 1.16 & 0.269 & 148.00 \\
\hline Cs & 38.4 & 1.5 & 41.1 & 1.05 & 7.08 & 39.2 & 3.39 & 2.16 & 0.97 & 0.13 & 1.11 & 0.0433 & 14.30 & 1.12 & 0.129 & 15.50 \\
\hline $\mathrm{Ba}$ & 343 & 45 & 314 & 7.62 & -8.42 & 317 & 8.09 & -7.60 & 1900 & 140 & 1860 & 46.9 & -2.13 & 1879 & 29.6 & -1.11 \\
\hline $\mathrm{La}$ & 54 & 5 & 48.4 & 4.86 & -10.33 & 52.2 & 2.8 & -3.42 & 60.5 & 5.2 & 57.5 & 2.53 & -4.98 & 59.5 & 1.77 & -1.68 \\
\hline $\mathrm{Ce}$ & 108 & 11 & 101 & 8.94 & -6.31 & 109 & 5.95 & 0.80 & 112 & 7 & 114 & 3.84 & 1.36 & 117 & 3.53 & 4.18 \\
\hline $\operatorname{Pr}$ & 12.7 & 0.8 & 11.5 & 1.27 & -9.51 & 12 & 0.689 & -5.16 & 13.2 & 0.8 & 12.7 & 0.446 & -4.12 & 12.9 & 0.468 & -2.42 \\
\hline $\mathrm{Nd}$ & 47 & 5 & 40.1 & 3.43 & -14.65 & 43.6 & 3.05 & -7.15 & 48.1 & 4.3 & 46.5 & 1.82 & -3.26 & 47.6 & 2.13 & -0.96 \\
\hline $\mathrm{Sm}$ & 9.7 & 1.2 & 8.82 & 0.831 & -9.12 & 9.37 & 0.825 & -3.40 & 7.74 & 0.48 & 7.25 & 0.43 & -6.29 & 7.67 & 0.535 & -0.94 \\
\hline $\mathrm{Eu}$ & 0.85 & 0.01 & 0.76 & 0.0398 & -10.53 & 0.86 & 0.0959 & 1.13 & 1.91 & 0.19 & 1.92 & 0.11 & 0.78 & 1.9 & 0.116 & -0.50 \\
\hline $\mathrm{Gd}$ & 9.3 & 0.8 & 8.28 & 0.657 & -10.95 & 8.53 & 0.783 & -8.28 & 5.09 & 0.44 & 4.87 & 0.339 & -4.24 & 5 & 0.423 & -1.77 \\
\hline $\mathrm{Tb}$ & 1.65 & 0.13 & 1.4 & 0.124 & -14.85 & 1.48 & 0.12 & -10.46 & 0.68 & 0.11 & 0.573 & 0.0356 & -15.74 & 0.563 & 0.0467 & -17.24 \\
\hline Dy & 10.2 & 0.04 & 9.23 & 0.805 & -9.46 & 9.89 & 0.676 & -3.01 & 3.2 & 0.34 & 3.17 & 0.221 & -0.85 & 3.18 & 0.225 & -0.63 \\
\hline Но & 2.05 & 0.22 & 1.92 & 0.12 & -6.16 & 2.04 & 0.14 & -0.72 & 0.6 & 0.17 & 0.595 & 0.0347 & -0.83 & 0.605 & 0.0439 & 0.77 \\
\hline $\mathrm{Er}$ & 6.5 & 0.4 & 6.1 & 0.525 & -6.14 & 6.56 & 0.454 & 0.89 & 1.57 & 0.11 & 1.6 & 0.122 & 1.73 & 1.6 & 0.143 & 2.02 \\
\hline $\mathrm{Tm}$ & 1.06 & 0.011 & 0.972 & 0.0663 & -8.27 & 1.06 & 0.103 & -0.37 & 0.26 & 0.03 & 0.227 & 0.0131 & -12.54 & 0.235 & 0.0288 & -9.67 \\
\hline $\mathrm{Yb}$ & 7.4 & 0.7 & 6.93 & 0.582 & -6.30 & 7.53 & 0.879 & 1.81 & 1.56 & 0.09 & 1.59 & 0.115 & 2.13 & 1.48 & 0.209 & -5.31 \\
\hline $\mathrm{Lu}$ & 1.15 & 0.12 & 1.03 & 0.0735 & -10.12 & 1.14 & 0.104 & -0.49 & 0.24 & 0.03 & 0.217 & 0.0158 & -9.68 & 0.223 & 0.03 & -6.95 \\
\hline $\mathrm{Hf}$ & 6.3 & 0.8 & 5.05 & 0.809 & -19.86 & 5.76 & 0.534 & -8.50 & 5.2 & 1.2 & 5.1 & 0.469 & -1.91 & 5.54 & 0.311 & 6.55 \\
\hline $\mathrm{Ta}$ & 7.2 & 0.7 & 6.59 & 0.447 & -8.45 & 7.08 & 0.581 & -1.61 & 0.62 & 0.15 & 0.564 & 0.0449 & -9.01 & 0.565 & 0.0272 & -8.85 \\
\hline $\mathrm{Pb}$ & 31 & 4 & 37.7 & 2.18 & 21.70 & - & - & - & 19.8 & 2.3 & 20 & 1.37 & - & - & - & - \\
\hline Th & 54 & 4 & 53.6 & 12.1 & -0.66 & 54.8 & 3.3 & 1.39 & 10.9 & 1 & 10.3 & 0.997 & -5.58 & 10.4 & 0.575 & -4.96 \\
\hline $\mathrm{U}$ & 18.8 & 2.2 & 20 & 3.35 & 6.28 & 18.2 & 1.71 & -3.25 & 1.4 & 0.35 & 1.53 & 0.222 & 9.62 & 1.53 & 0.08 & 9.01 \\
\hline
\end{tabular}


Chapter 3.1 Comparison of Ultrafine Powder Pellet and Flux-free Fusion Glass for Bulk Analysis of Granitic Rock Samples by Laser Ablation-Inductively Coupled Plasma-Mass Spectrometry

Table 5 Analytical results of JG-2 and JG-3 obtained from powder pellet and flux-free fusion glass techniques. The data are complied with NIST610, StHs6/80-G and GSD-1G as external reference materials.

\begin{tabular}{|c|c|c|c|c|c|c|c|c|c|c|c|c|c|c|c|c|}
\hline \multirow[b]{3}{*}{ Elements } & \multicolumn{8}{|c|}{ JG-2 } & \multicolumn{8}{|c|}{ JG-3 } \\
\hline & \multicolumn{2}{|c|}{ Reference value } & \multicolumn{3}{|c|}{ Ultrafine powder pellet } & \multicolumn{3}{|c|}{ Flux-free fusion glass } & \multicolumn{2}{|c|}{ Reference value } & \multicolumn{3}{|c|}{ Ultrafine powder pellet } & \multicolumn{3}{|c|}{ Flux-free fusion glass } \\
\hline & Value & Uncertainty & Average & $1 \mathrm{~s}$ & $\mathrm{RD}(\%)$ & Average & $1 \mathrm{~s}$ & $\mathrm{RD}(\%)$ & Value & Uncertainty & Average & $1 \mathrm{~s}$ & $\mathrm{RD}(\%)$ & Average & $1 \mathrm{~s}$ & $\mathrm{RD}(\%)$ \\
\hline $\mathrm{SiO}_{2}$ & 76.8 & 0.57 & - & - & - & 75.5 & 0.398 & -1.79 & 67.3 & 0.55 & - & - & - & 69.3 & 0.505 & 3.04 \\
\hline $\mathrm{TiO}_{2}$ & 0.044 & 0.009 & 0.042 & 0.0011 & -4.52 & 0.0443 & 0.0027 & 0.62 & 0.48 & 0.03 & 0.463 & 0.0168 & -3.59 & 0.48 & 0.018 & 0.09 \\
\hline $\mathrm{Al}_{2} \mathrm{O}_{3}$ & 12.5 & 0.32 & 12.5 & 0.405 & 0.00 & 12.5 & 0.287 & 0.00 & 15.5 & 0.2 & 15.5 & 0.513 & 0.00 & 15.5 & 0.336 & 0.00 \\
\hline $\mathrm{FeO}(\mathrm{t})$ & 0.867 & 0.15 & 0.878 & 0.0322 & 1.23 & 0.866 & 0.0269 & -0.16 & 3.29 & 0.15 & 3.41 & 0.204 & 3.57 & 3.57 & 0.0531 & 8.67 \\
\hline $\mathrm{MnO}$ & 0.016 & 0.003 & 0.0171 & 0.0004 & 6.89 & 0.0176 & 0.0007 & 10.10 & 0.071 & 0.004 & 0.0731 & 0.0024 & 2.94 & 0.0753 & 0.0011 & 6.03 \\
\hline $\mathrm{MgO}$ & 0.037 & 0.012 & 0.0396 & 0.001 & 6.91 & 0.0477 & 0.0102 & 28.90 & 1.79 & 0.06 & 1.7 & 0.121 & -4.80 & 1.77 & 0.0154 & -1.27 \\
\hline $\mathrm{CaO}$ & 0.7 & 0.05 & 0.761 & 0.0663 & 8.70 & 0.768 & 0.155 & 9.76 & 3.69 & 0.09 & 3.85 & 0.131 & 4.28 & 3.85 & 0.119 & 4.40 \\
\hline $\mathrm{Na}_{2} \mathrm{O}$ & 3.54 & 0.11 & 3.63 & 0.128 & 2.56 & 3.33 & 0.0927 & -5.87 & 3.96 & 0.15 & 4.03 & 0.142 & 1.81 & 4.06 & 0.0422 & 2.45 \\
\hline $\mathrm{K}_{2} \mathrm{O}$ & 4.71 & 0.09 & 4.91 & 0.149 & 4.32 & 4.46 & 0.216 & -5.27 & 2.64 & 0.04 & 2.85 & 0.252 & 8.10 & 2.75 & 0.0503 & 4.23 \\
\hline $\mathrm{P}_{2} \mathrm{O}_{5}$ & 0.002 & 0.0001 & 0.0035 & 0.0013 & 76.50 & 0.0075 & 0.0022 & 273.00 & 0.122 & 0.011 & 0.127 & 0.0095 & 4.14 & 0.122 & 0.0112 & 0.21 \\
\hline $\mathrm{Li}$ & 42.2 & 3.1 & 41.5 & 2.39 & -1.65 & 39.5 & 4.89 & -6.30 & 20.9 & 1.6 & 21.9 & 1.75 & 4.87 & 22.6 & 2 & 8.36 \\
\hline $\mathrm{Be}$ & 3.26 & 0.52 & 3.42 & 1 & 4.87 & 4.75 & 2.51 & 45.60 & 1.6 & 0.29 & 1.76 & 0.498 & 10.20 & 1.15 & 1.75 & -28.14 \\
\hline $\mathrm{Sc}$ & 2.42 & 0.42 & 3.7 & 0.192 & 53.00 & 3.57 & 0.496 & 47.60 & 8.76 & 0.55 & 9.6 & 0.603 & 9.58 & 9.89 & 0.771 & 12.90 \\
\hline $\mathrm{V}$ & 3.78 & 1.58 & 0.752 & 0.148 & -80.11 & 0.836 & 0.259 & -77.88 & 70.1 & 6.2 & 72.8 & 5.66 & 3.92 & 72.4 & 3.34 & 3.21 \\
\hline $\mathrm{Cr}$ & 6.37 & 2.09 & 3.1 & 0.317 & -51.28 & 3.67 & 1.7 & -42.35 & 22.4 & 3.5 & 23.5 & 4.85 & 4.70 & 19 & 1.85 & -15.04 \\
\hline Co & 3.62 & 0.83 & 4.23 & 0.159 & 17.00 & 4.12 & 0.328 & 13.80 & 11.7 & 1.2 & 11.2 & 0.285 & -4.57 & 11.7 & 0.478 & -0.13 \\
\hline $\mathrm{Ni}^{*}$ & 4.35 & 3.07 & 2.48 & 0.641 & -43.05 & - & - & - & 14.3 & 2.2 & 13.9 & 1.37 & -2.81 & - & - & - \\
\hline $\mathrm{Cu}$ & 0.49 & 0.12 & 1.22 & 0.639 & 149.00 & 4.45 & 1.02 & 807.00 & 6.81 & 1.14 & 6.25 & 1.6 & -8.17 & 8.36 & 0.535 & 22.80 \\
\hline $\mathrm{Zn}$ & 13.6 & 2.4 & 13.7 & 2.2 & 0.93 & 11.7 & 2.43 & -14.27 & 46.5 & 3.8 & 58.9 & 2.71 & 26.80 & 52.6 & 3.34 & 13.20 \\
\hline $\mathrm{Ga}$ & 18.6 & 0.74 & 14.8 & 0.632 & -20.26 & 17.2 & 1.97 & -7.29 & 17.1 & 1.5 & 16.3 & 0.56 & -4.89 & 16.2 & 1.31 & -5.22 \\
\hline $\mathrm{Rb}$ & 301 & 12 & 309 & 13.9 & 2.56 & 288 & 11.3 & -4.18 & 67.3 & 7.3 & 73.9 & 3.1 & 9.86 & 72.6 & 3.1 & 7.90 \\
\hline $\mathrm{Sr}$ & 17.9 & 3.5 & 15.7 & 0.723 & -12.22 & 16.7 & 0.788 & -6.56 & 379 & 29 & 362 & 11 & -4.40 & 368 & 9.69 & -2.80 \\
\hline $\mathrm{Y}$ & 86.5 & 8 & 72.7 & 5.16 & -15.97 & 80.5 & 3.27 & -6.94 & 17.3 & 1.5 & 15.2 & 0.61 & -11.86 & 15.3 & 0.706 & -11.33 \\
\hline $\mathrm{Zr}$ & 97.6 & 10.8 & 92.2 & 17.3 & -5.58 & 96.3 & 24.3 & -1.35 & 144 & 12 & 143 & 16.3 & -1.01 & 153 & 4.78 & 6.35 \\
\hline $\mathrm{Nb}$ & 14.7 & 1.4 & 14.1 & 1.12 & -4.14 & 15.3 & 1.22 & 4.19 & 5.88 & 0.85 & 6.27 & 0.701 & 6.67 & 6.28 & 0.486 & 6.73 \\
\hline Mo & 0.37 & 0.16 & 0.213 & 0.0835 & -42.47 & 0.41 & 0.239 & 10.80 & 0.45 & 0.085 & 0.204 & 0.0508 & -54.74 & 0.532 & 0.112 & 18.20 \\
\hline $\mathrm{Cs}$ & 6.79 & 0.088 & 7.59 & 0.292 & 11.80 & 7.13 & 0.688 & 4.94 & 1.78 & 0.22 & 2.19 & 0.0804 & 22.80 & 2.13 & 0.118 & 19.70 \\
\hline $\mathrm{Ba}$ & 58 & 30 & 54.3 & 2.31 & -6.41 & 58.9 & 3.07 & 1.48 & 466 & 44 & 456 & 16.8 & -2.09 & 459 & 9.05 & -1.55 \\
\hline $\mathrm{La}$ & 19.9 & 2.6 & 16.8 & 1.31 & -15.38 & 19.1 & 0.731 & -4.11 & 20.6 & 2.2 & 21.1 & 2.86 & 2.57 & 20.9 & 0.624 & 1.32 \\
\hline $\mathrm{Ce}$ & 48.3 & 5.3 & 43.2 & 2.72 & -10.49 & 48.3 & 2.2 & 0.09 & 40.3 & 4.8 & 42.7 & 4.47 & 5.88 & 42.2 & 1.43 & 4.61 \\
\hline $\operatorname{Pr}$ & 6.2 & 1.15 & 5.3 & 0.253 & -14.47 & 5.97 & 0.375 & -3.72 & 4.7 & 1.24 & 4.69 & 0.384 & -0.20 & 4.62 & 0.183 & -1.66 \\
\hline $\mathrm{Nd}$ & 26.4 & 5.2 & 21.6 & 1.34 & -18.34 & 25.2 & 1.88 & -4.52 & 17.2 & 1.8 & 16.8 & 1.01 & -2.11 & 17.5 & 0.987 & 2.01 \\
\hline $\mathrm{Sm}$ & 7.78 & 1.1 & 6.96 & 0.409 & -10.51 & 7.81 & 0.793 & 0.41 & 3.39 & 0.44 & 3.38 & 0.341 & -0.24 & 3.2 & 0.33 & -5.61 \\
\hline $\mathrm{Eu}$ & 0.1 & 0.044 & 0.0858 & 0.0155 & -14.20 & 0.14 & 0.0695 & 39.60 & 0.9 & 0.077 & 0.793 & 0.0462 & -11.85 & 0.833 & 0.0538 & -7.49 \\
\hline $\mathrm{Gd}$ & 8.01 & - & 8.37 & 0.498 & 4.48 & 9.48 & 0.891 & 18.40 & 2.92 & 0.28 & 3 & 0.221 & 2.80 & 2.9 & 0.24 & -0.67 \\
\hline $\mathrm{Tb}$ & 1.62 & 0.32 & 1.54 & 0.084 & -5.20 & 1.77 & 0.145 & 9.45 & 0.46 & 0.05 & 0.412 & 0.0265 & -10.48 & 0.39 & 0.026 & -15.15 \\
\hline Dy & 10.5 & 2.3 & 11.2 & 0.758 & 6.67 & 12.3 & 0.794 & 16.90 & 2.59 & 0.53 & 2.65 & 0.138 & 2.43 & 2.63 & 0.178 & 1.48 \\
\hline Но & 2.9 & - & 2.35 & 0.134 & -19.00 & 2.67 & 0.184 & -7.82 & 0.38 & 0.16 & 0.528 & 0.0222 & 38.80 & 0.553 & 0.0487 & 45.50 \\
\hline $\mathrm{Er}$ & 6.04 & 1.88 & 7.26 & 0.603 & 20.30 & 8.18 & 0.477 & 35.50 & 1.52 & 0.36 & 1.6 & 0.105 & 5.53 & 1.62 & 0.083 & 6.59 \\
\hline $\mathrm{Tm}$ & 1.16 & 0.74 & 1.13 & 0.0658 & -2.79 & 1.29 & 0.206 & 11.10 & 0.24 & 0.048 & 0.237 & 0.0187 & -1.19 & 0.244 & 0.0279 & 1.46 \\
\hline $\mathrm{Yb}$ & 6.85 & 2.15 & 7.81 & 0.656 & 14.00 & 8.99 & 1.26 & 31.20 & 1.77 & 0.35 & 1.7 & 0.0812 & -4.02 & 1.65 & 0.163 & -6.97 \\
\hline $\mathrm{Lu}$ & 1.22 & 0.14 & 1.11 & 0.0839 & -9.22 & 1.25 & 0.125 & 2.80 & 0.26 & 0.049 & 0.262 & 0.0183 & 0.87 & 0.272 & 0.0324 & 4.78 \\
\hline $\mathrm{Hf}$ & 4.73 & 1.3 & 4.56 & 1.23 & -3.54 & 5.02 & 2 & 6.08 & 4.29 & 0.41 & 3.94 & 0.363 & -8.07 & 4.2 & 0.289 & -2.07 \\
\hline $\mathrm{Ta}$ & 2.25 & - & 1.95 & 0.164 & -13.16 & 2.36 & 0.234 & 4.69 & 0.7 & 0.11 & 0.552 & 0.046 & -21.13 & 0.605 & 0.09 & -13.56 \\
\hline $\mathrm{Pb}$ & 31.5 & 4.1 & 29.8 & 3.47 & -5.30 & - & - & - & 11.7 & 1.4 & 12.6 & 0.649 & 7.30 & - & - & - \\
\hline Th & 31.6 & 2.7 & 28.2 & 4.51 & -10.77 & 32 & 1.85 & 1.17 & 8.28 & 0.65 & 8.06 & 2.86 & -2.67 & 8.17 & 0.409 & -1.34 \\
\hline $\mathrm{U}$ & 11.3 & 1.6 & 11 & 0.866 & -2.60 & 10.1 & 0.512 & -10.81 & 2.21 & 0.41 & 2.39 & 0.528 & 8.35 & 2.34 & 0.104 & 5.86 \\
\hline
\end{tabular}


Chapter 3.1 Comparison of Ultrafine Powder Pellet and Flux-free Fusion Glass for Bulk Analysis of Granitic Rock Samples by Laser Ablation-Inductively Coupled Plasma-Mass Spectrometry

The milling time should be long enough to generate ultrafine powders, while too long milling time will increase the abrasion of agate materials. Our experiments to optimize the milling time in order to produce a sufficiently fine powder but to avoid excessive abrasion of agate materials suggested $45 \mathrm{~min}$ as the compromise milling time, which was used in all subsequent milling procedures (Fig.2b).
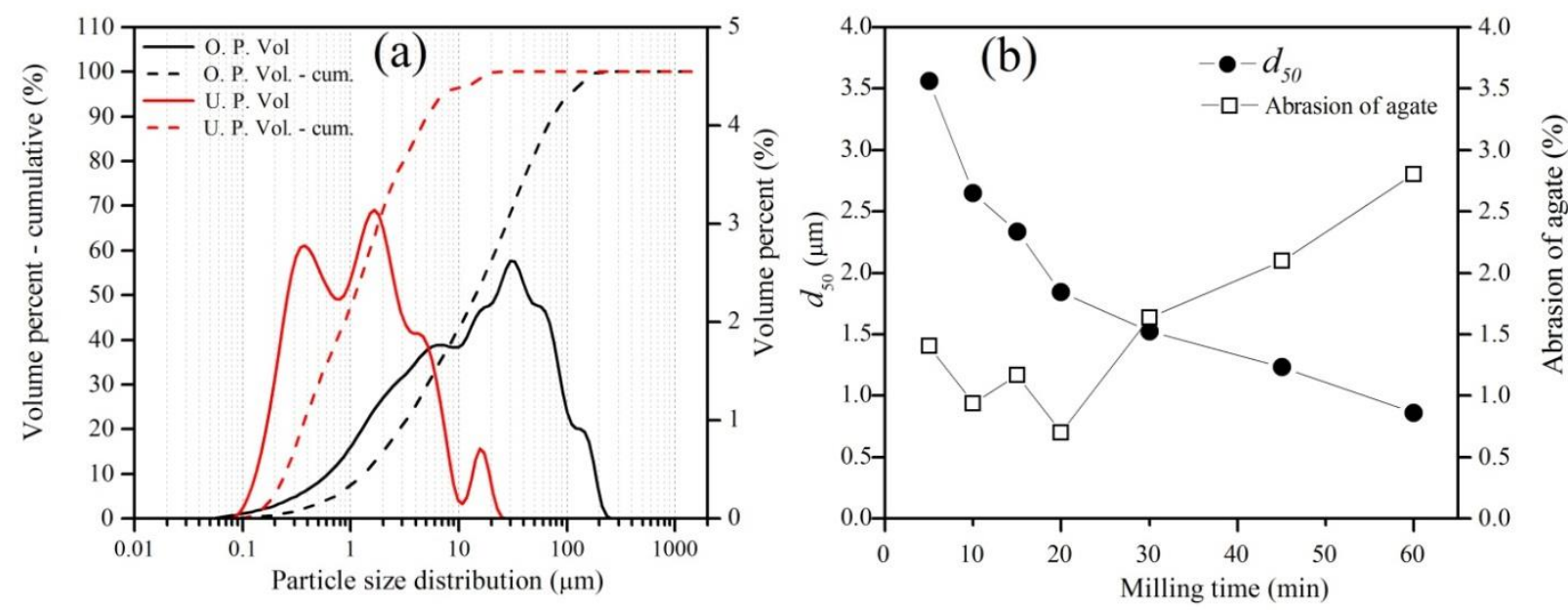

Fig.2 (a) grain size distribution of original and wet-milled granitic powder (G1RF) at a milling time of 45 min. (b) the $\mathrm{d}_{50}(\mu \mathrm{m})$ and the abrasion $(\%)$ of agate as function of milling time. "O.P" represents original powders. "U.P." represents the ultrafine powders. The abrasion of agate ball is given as wt \% relative the mass of the original rock powders.

The particles were characterized as $\mathrm{d}_{50}=1.2 \mu \mathrm{m}, \mathrm{d}_{90}=5.5 \mu \mathrm{m}$ (Fig.2), which is comparable to previous results $\left(\mathrm{d}_{50}=1.4 \mu \mathrm{m}, \mathrm{d}_{90}=7.9 \mu \mathrm{m}\right)($ Garbe-Schönberg and Müller 2014). The slightly smaller grain size in our powders may be due to the use of the smaller $5 \mathrm{~mm}$ balls since finer particles are generally obtained with smaller balls. The grain size distribution curve of the ultrafine powder (Fig.2a) shows polymodal behavior with three modal values at $0.35 \mu \mathrm{m}, 1.5 \mathrm{um}$ and $15 \mu \mathrm{m}$ in our powders. To further characterize the according to particles, we conducted the SEM imaging (Appendix S2) and EMPA elemental mapping (Fig.3). The results show that the particles are significantly decreased after wet-milling compared to the original powders (Appendix S2). EMPA mapping shows that some larger particle (up to $15 \mu \mathrm{m}$ ) survived the wet-milling procedure (Fig.3). Based on the mineral chemistry, these large particles were identified as biotite. These sheet silicates might be extremely sticky when milled together with more isometric grains and therefore hard to decrease in grain size. 
Chapter 3.1 Comparison of Ultrafine Powder Pellet and Flux-free Fusion Glass for Bulk Analysis of Granitic Rock Samples by Laser Ablation-Inductively Coupled Plasma-Mass Spectrometry

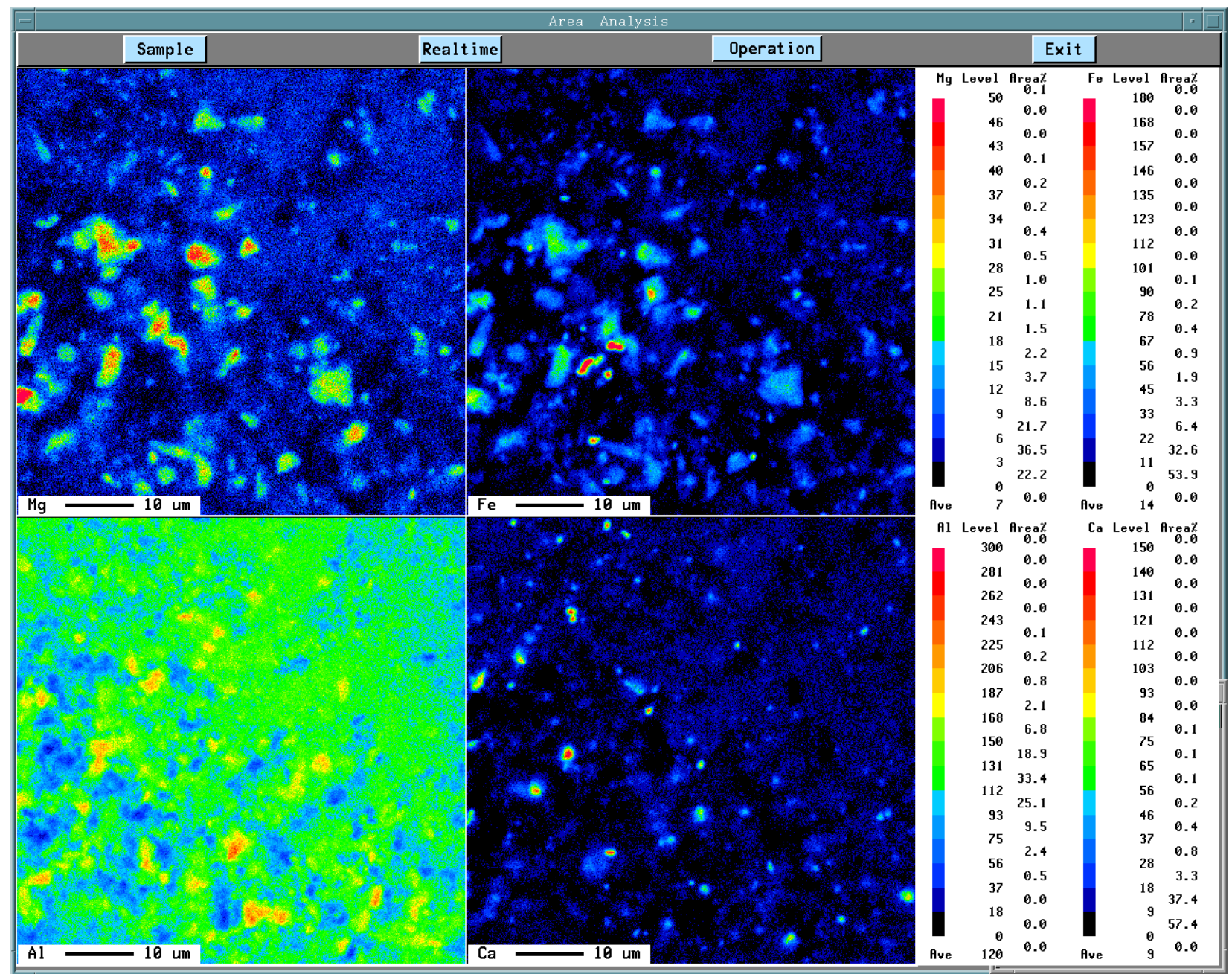

Fig.3 EMPA mapping image of ultra-fine powder pellets (G1RF)

The contaminations during the wet-milling process mainly stem from two sources: a. the addition of high-purity water, b. the abrasion of agate materials. Peters and Pettke (2017) systematically investigated the contaminations caused by the addition of high-purity water and demonstrated that considerable contaminations of $\mathrm{B}, \mathrm{Cu}$, and $\mathrm{Zn}$ were introduced by the high-purity water. These elements should be carefully checked when evaluating the LA-ICP-MS analytical data of powder pellets produced by wet milling. The same study found that the abrasion of agate balls should be insignificant. However, this is not consistent with our results, which show that around 2-3\% agate materials relative to $2.78 \mathrm{~g}$ sample are introduced into the original samples during the wet-milling process (data not shown). Thus, the potential contaminations caused by the agate balls should be considered. We analyzed the major and trace element contents in the agate balls by LA-ICP-MS (calibrated with NIST610). The results illustrate that the agate balls are two magnitudes lower in all 
Chapter 3.1 Comparison of Ultrafine Powder Pellet and Flux-free Fusion Glass for Bulk Analysis of Granitic Rock Samples by Laser Ablation-Inductively Coupled Plasma-Mass Spectrometry

chemical components relative to the granite samples (GSP-2 and GBW07111), except for elements Si, $\mathrm{B}, \mathrm{Cu}, \mathrm{Sb}$ and $\mathrm{Tl}$ (Appendix S3), which reveals that the agate balls are "clean" compared to the granite samples. The addition of abraded agate materials will dilute the concentrations in the sample powders by around $3 \%$.

\subsubsection{Optimization of flux-free fusion protocol}

To produce homogeneous glass from granitic samples without adding any flux is challenging due to the difficulty of completely dissolving refractory minerals (e.g. zircon) and due to slow chemical diffusion and homogenization in high viscosity melts. Previous results (He et al. 2016) indicate that complete dissolution of zircon in granitic samples melt did not occur even after 30 min of fusion at $1600{ }^{\circ} \mathrm{C}$. Temperature lower than $1600{ }^{\circ} \mathrm{C}$ is thus insufficient to dissolve refractory minerals, while temperature higher than $1600{ }^{\circ} \mathrm{C}$ would result in the heavy loss of highly volatile elements (Fedorowich et al. 1993, Stoll et al. 2008). Therefore in this study, the melting temperature was fixed at $1600{ }^{\circ} \mathrm{C}$. GBW 07103 and JG-2 were used to investigate glass homogeneity and the behavior of volatile elements; hence it is possible to directly compare the data from fusion experiment with reference values.

Homogeneity is a crucial parameter for the bulk analysis using any microanalytical technique. Chemical diffusion in high viscosity melt is considered as one of the key factors controlling the glass homogeneity. Considering the slow chemical diffusion in granitic melts, an increase of melting time (Zhang et al. 2016) or a re-grinding step (Reid et al. 1999) are necessary to obtain homogeneous glasses. Here we carried out experiments to understand how the glass homogeneity is improved with increasing melting time (from 1 to 24 hours). The results illustrate that the glass homogeneity was slightly improved with the increase of melting time from 1 to $4 \mathrm{~h}$, however, this slight improvement is still not sufficient for achieving representative sampling for LA-ICP-MS, especially with less number of analyses (e.g. n=3) (Fig.4). Further increase of melting time is not recommended since it would result in the increased depletion of highly volatile elements (Fig.5). Our experience illustrates that homogeneous glasses from granitic samples could not be produced even after 24 hours of melting at $1600{ }^{\circ} \mathrm{C}$ without grinding. This demonstrated the slow chemicals diffusion in granitic melts, especially for these high valence elements Sr, Y and Zr. Figure 4 reveals that the re-grinding step (e.g. 
Chapter 3.1 Comparison of Ultrafine Powder Pellet and Flux-free Fusion Glass for Bulk Analysis of Granitic Rock Samples by Laser Ablation-Inductively Coupled Plasma-Mass Spectrometry

mechanical homogenization) significantly improved the homogeneity of the glass at a short melting time $(<2 \mathrm{~h})$.

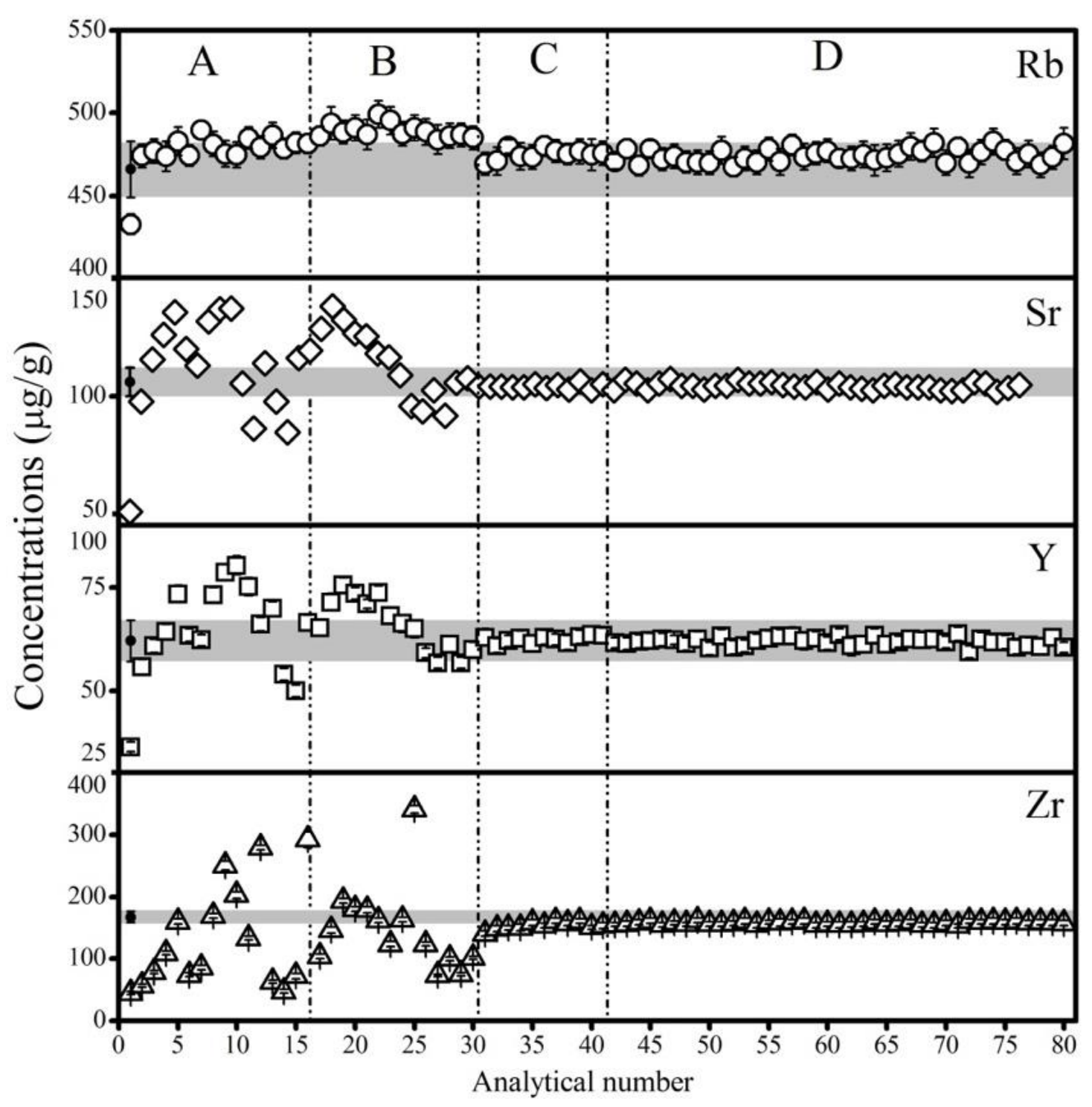

Fig.4 Homogeneity assessment for the glasses (GBW07103) produced at different fusion conditions, A. $1 \mathrm{~h}$ melting without grinding, B. $4 \mathrm{~h}$ melting without grinding, C. $2 \mathrm{~h}$ melting with one grinding, D. $4 \mathrm{~h}$ melting with two grindings. The results are quantified by using $\mathrm{Al}$ as internal standard and NIST610 as external reference material. Assessment of the glass homogeneity is based on the variability of $\mathrm{Rb}, \mathrm{Sr}, \mathrm{Y}$ and $\mathrm{Zr}$ concentrations. The error bars (2s) are derived from single spot analysis and are generally smaller than symbol sizes. Grey zone represents the reference values with $95 \%$ confidence. 


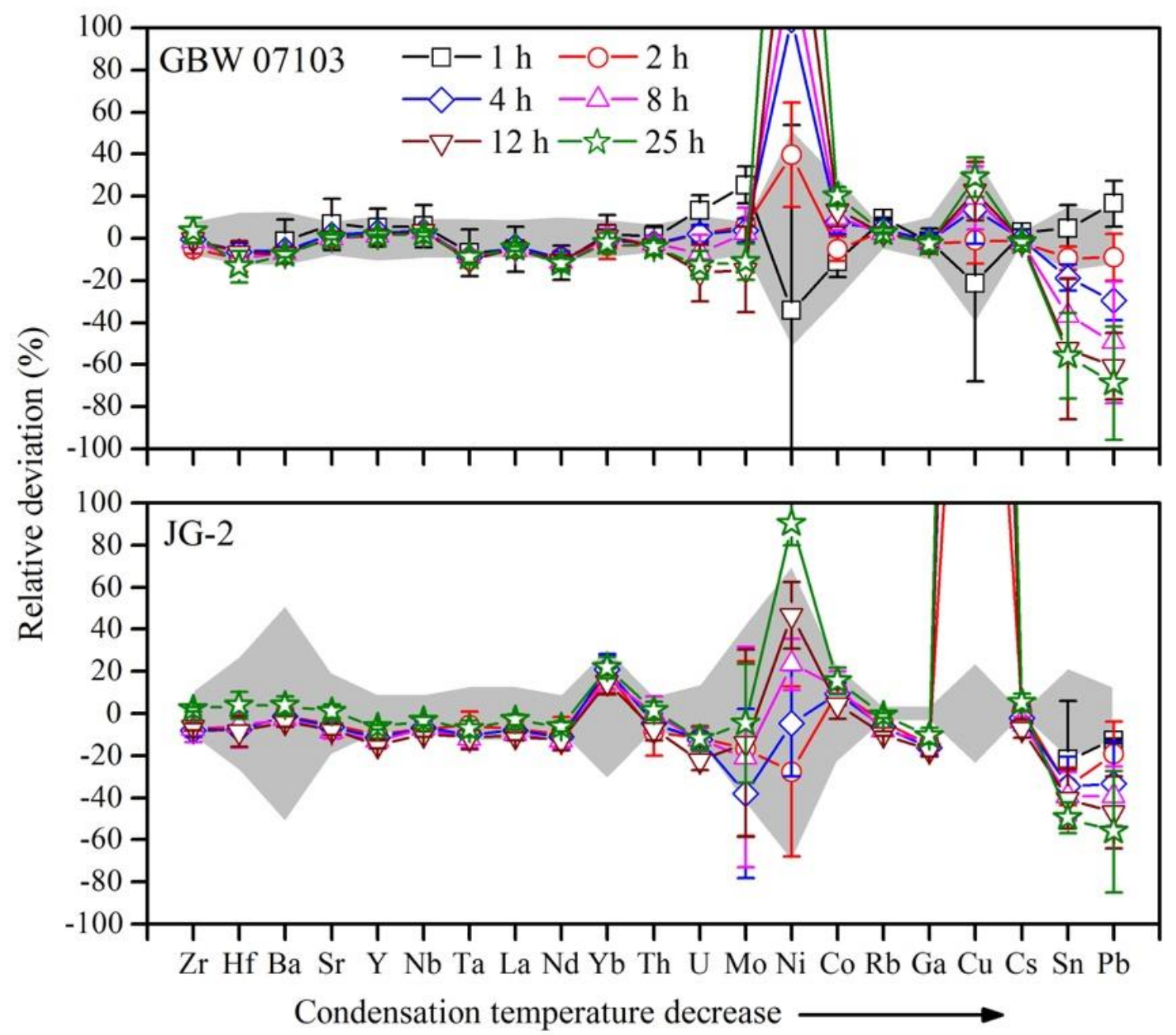

Fig.5 The deviation of trace element concentrations in fusion glasses relative to certified values. Six different melting conditions (including $1 \mathrm{~h}, 2 \mathrm{~h}, 4 \mathrm{~h}, 8 \mathrm{~h}, 12 \mathrm{~h}$ and $25 \mathrm{~h}$ ) were used to investigate the behavior of volatile elements. Elements are arranged according their volatility based on their condensation temperatures (from Wasson 1985). The results are quantified by using $\mathrm{Al}$ as internal standard and NIST610 as external reference material. Some data of one hour melting condition are not plotted due to highly heterogeneity of glasses. 


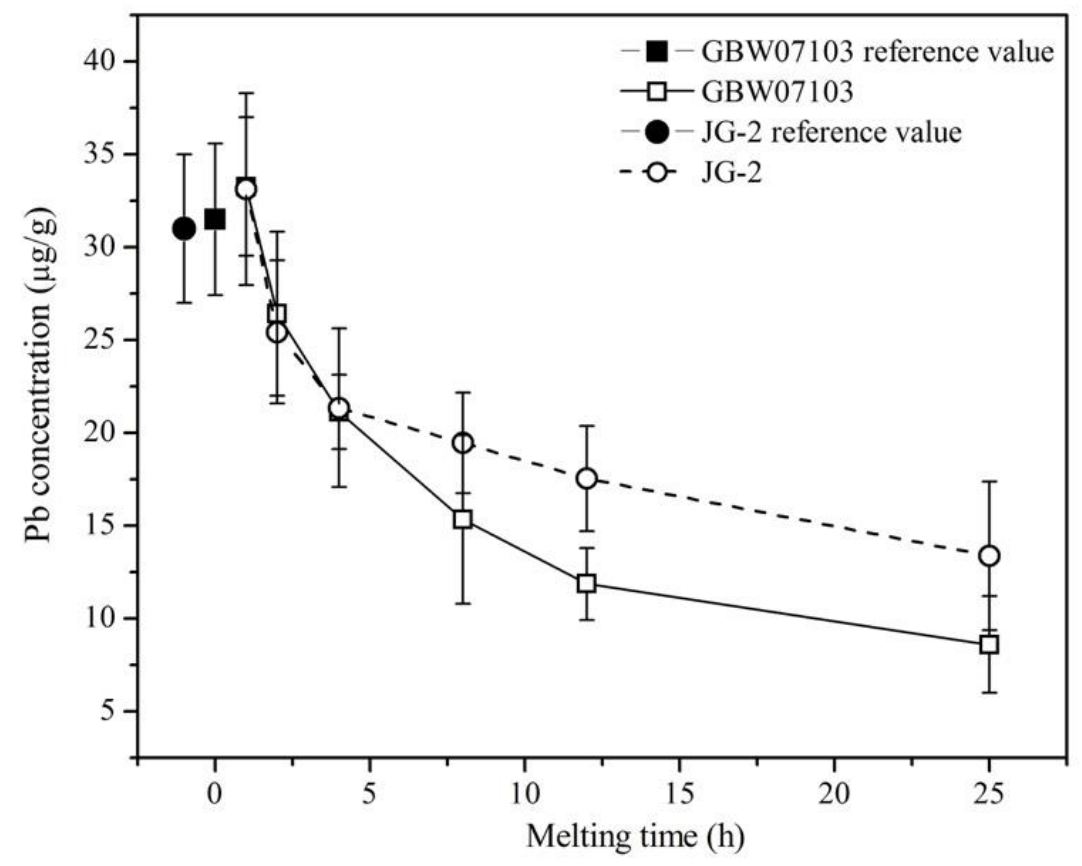

Fig.6 Depletion of volatile $\mathrm{Pb}$ in GBW 07103 and JG-2 with increase of melting time.

The depletion of highly volatile elements (e.g. Pb) during fusion at a high temperature (e.g. $1600{ }^{\circ} \mathrm{C}$ ) was reported in previous studies (Fedorowich et al. 1993, Stoll et al. 2008, Zhang et al. 2016). In this study, we used an open platinum crucible covered with a lid; hence the depletions of highly volatile elements are expected. Figure 5 shows the relative deviation of trace element concentration to certified values in fusion glasses at the different melting time (1 h, $2 \mathrm{~h}, 4 \mathrm{~h}, 8 \mathrm{~h}, 12 \mathrm{~h}$ and $25 \mathrm{~h})$. Elements are arranged according to their volatility based on the condensation temperatures at a pressure of $10^{-4}$ bar (Wasson 1985). The data illustrate that the highly volatile elements (such as $\mathrm{Sn}$ and $\mathrm{Pb}$ ) are depleted with increasing melting time, up to more than $60 \%$ at $25 \mathrm{~h}$ (Fig. 5). Less volatile elements such as $\mathrm{Rb}$ and $\mathrm{Cs}$ were not significantly depleted using our fusion technique. Figure 6 reveals the variation of $\mathrm{Pb}$ concentration in GBW 07103 and JG-2 as a function of melting time. The relative stronger depletion of $\mathrm{Pb}$ in GBW 07103 compared to JG-2 may be related to the lower viscosity of GBW 07103 melt. The Pb concentration decreases towards the melt surface (Fig.7), which is probably caused by the usage of an open platinum crucible; therefore the sampling position for highly volatile elements should preferably be at the glass in the bottom of the crucible. The increase of $\mathrm{Ni}$ concentration with melting time is probably caused by contaminations from the platinum crucible. 
Chapter 3.1 Comparison of Ultrafine Powder Pellet and Flux-free Fusion Glass for Bulk Analysis of Granitic Rock Samples by Laser Ablation-Inductively Coupled Plasma-Mass Spectrometry

Previous studies reported that the depletion of highly volatile elements can occur in a matter of tens of second. In this study, significant depletion of highly volatile elements occurred after two hours of fusion at $1600{ }^{\circ} \mathrm{C}$. This could be interpreted in two ways. First, large amounts of samples (several grams) were used in this studies, thus the depletion derived by the diffusion will be less compared to previous studies that produced small glass beads from milligram amounts of powders of mafic rocks. Another explanation could be that granitic melts investigated here have higher viscosity compared basaltic materials, which would limit the diffusion derived volatile depletion from the melt.
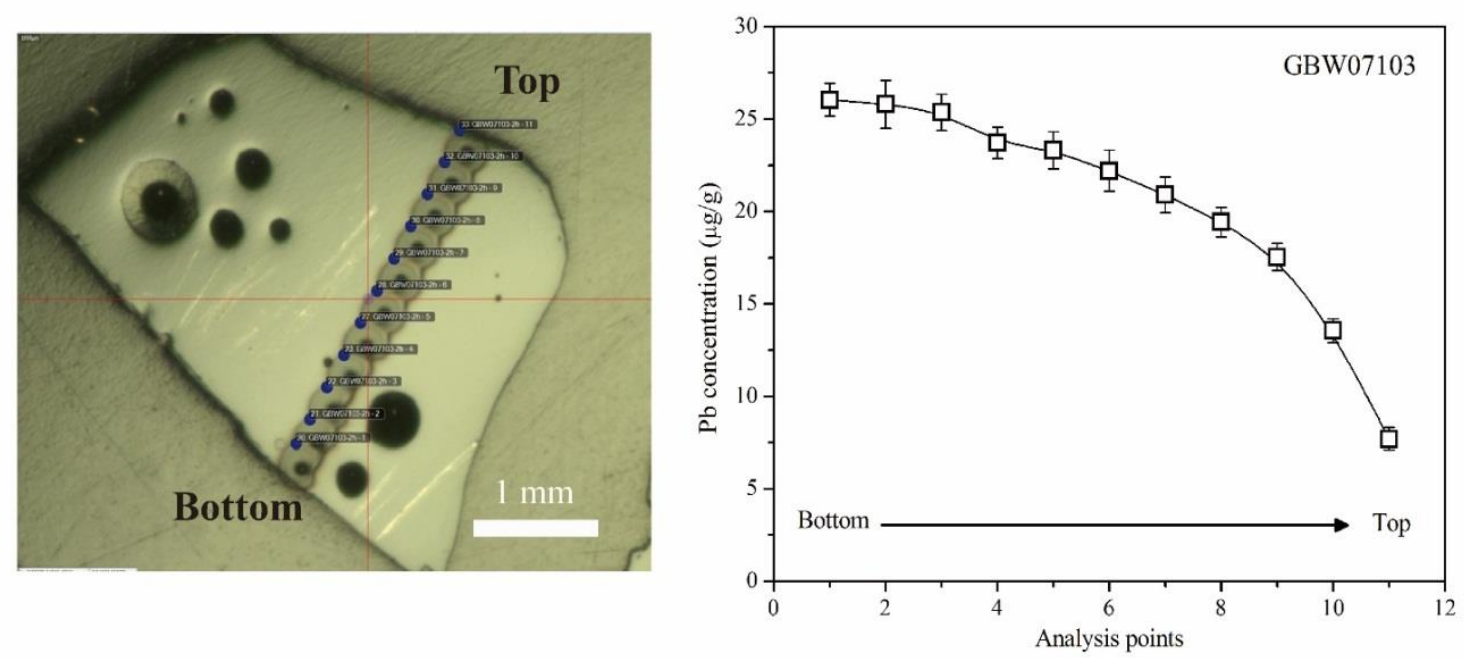

Fig.7 Gradient of decreasing $\mathrm{Pb}$ towards the melt surface

Our procedure for making homogeneous granitic glasses $\left(160{ }^{\circ} \mathrm{C}, 2 \mathrm{~h}\right.$ melting, 15 min re-grinding of the original glass) represents a compromise, as the glasses are homogeneous enough for representative sampling by LA-ICP-MS, and at the same time, highly volatile elements are not significantly depleted. Due to the higher $\mathrm{SiO} 2$ content, homogeneous glasses were not achieved for JG-2, thus the melting condition $\left(1600{ }^{\circ} \mathrm{C}, 2 \mathrm{~h}\right.$ melting, 2 times 15 min re-grinding) was applied for JG-2.

\subsubsection{Comparison of ablation behaviors}

Ablation behaviors significantly affect the quality of LA-ICP-MS analyses. Here the ablation rates and mass response of ultrafine powder pellets and the fusion glasses are compared in Fig.8 and Fig.9. NIST glasses, including dark NIST610, blue NIST612, and transparent NIST614, had similar ablation rates, which demonstrate that the $193 \mathrm{~nm}$ laser absorption efficiency is independent of the glass transparency. That is consistent with previous results (Horn et al. 2001). Systematically lower ablation 
Chapter 3.1 Comparison of Ultrafine Powder Pellet and Flux-free Fusion Glass for Bulk Analysis of Granitic Rock Samples by Laser Ablation-Inductively Coupled Plasma-Mass Spectrometry

rates were observed for geological glass compared to NIST glasses. Hu et al. (2011b) revealed similar results and reported that the ablation rate of NIST610 was 1.5 times higher than GSE-1G. The ablation rate of JG-2 was slightly lower than other geological glasses.

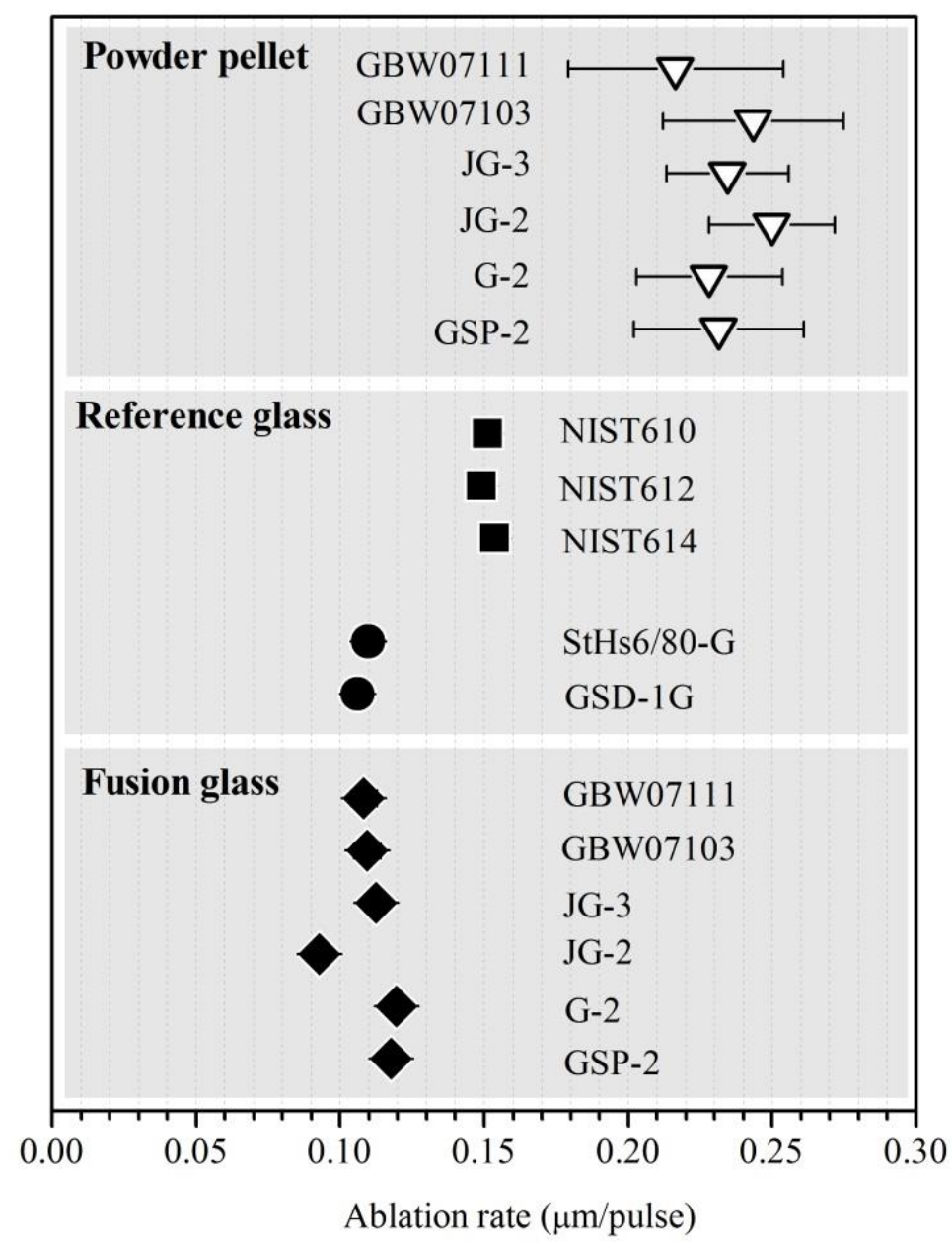

Fig.8 Ablation rates of reference materials (NIST glasses, StHs6/80-G and GSD-1G), fusion glasses and powder pellets. The error bars are derived from eight analyses

To understand the different ablation rates between geological glasses, a further study needs to be carried out. The powder pellets had relatively large ablation rates probably due to the low mechanical resistance of powder pellets.

Figure 9 reveals the mass response of La in the fusion glasses and powder pellets. To compare, the mass response of NIST610, StHs6/80-G, and GSD-1G were also plotted. The mass response of NIST610 was around 1.5 times higher than in geological glass. Considering 1.5 times higher ablation rates of NIST610, we conclude that the absolute ablated mass amount of NIST610 is 1.5 times higher 
Chapter 3.1 Comparison of Ultrafine Powder Pellet and Flux-free Fusion Glass for Bulk Analysis of Granitic Rock Samples by Laser Ablation-Inductively Coupled Plasma-Mass Spectrometry

than geological glasses. The reason for this difference is unclear but should be related to the matrix effect during ablation. Compared to glasses, powder pellets have a relatively low mass response, which is probably related to the lower density of powder pellets or the incomplete ionization of larger particles in the ICP. Ablation yields vary by as much as $100 \%$ among NIST glasses, geological glasses, and powder pellets, thus the internal standardization with a known internal standard is necessary to correct the difference in ablation efficiency, especially when non-matrix matched calibration is applied.

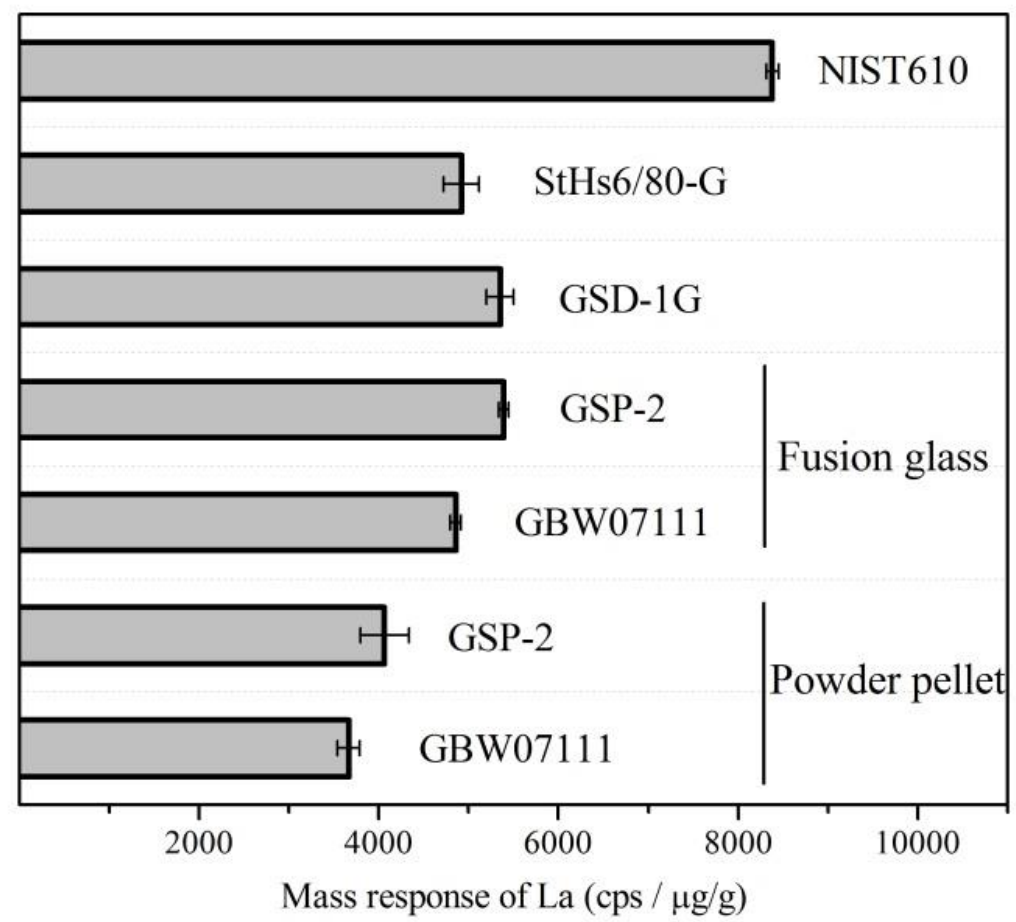

Fig.9 ICP mass response of La for reference materials (NIST610, StHs6/80-G and GSD-1G), fusion glass (GSP-2, GBW07103) as well as powder pellets (GSP-2, GBW07103). The signal intensities are corrected by the isotopic abundance.

\subsubsection{Comparison of analytical precision}

The analytical precision of the LA-ICP-MS data is highly dependent on the quality of powder pellets and fusion glasses, in particular with respect to their chemical homogeneity (Hu et al. 2011a, Yang et al. 2012), and is also affected by counting statistics (Gao et al. 2002, Hu et al. 2009, Wu et al. 2016). To evaluate the analytical precision of powder pellets and fusion glasses, we took MPI-DING glasses as reference. MPI-DING glasses have been intensively investigated in previous studies (Jochum et al. 
Chapter 3.1 Comparison of Ultrafine Powder Pellet and Flux-free Fusion Glass for Bulk Analysis of Granitic Rock Samples by Laser Ablation-Inductively Coupled Plasma-Mass Spectrometry

2000, Jochum et al. 2006) and widely accepted as homogeneous materials for LA-ICP-MS calibration.

Figure 10 illustrates that there is - as expected - a significant negative log-linear correlation between elemental concentration and their RSD for MPI-DING glasses. The trend follows the Poisson counting uncertainty, which reveals that the analytical precision is derived from the original analysis (counting statistics). Our data further demonstrate the homogeneity of MPI-DING glasses. The analytical precisions given as one-time RSD is less than $10 \%$ for most elements with concentrations higher than $0.1 \mu \mathrm{g} / \mathrm{g}$, which is approving acceptable for trace element determinations in geological samples.

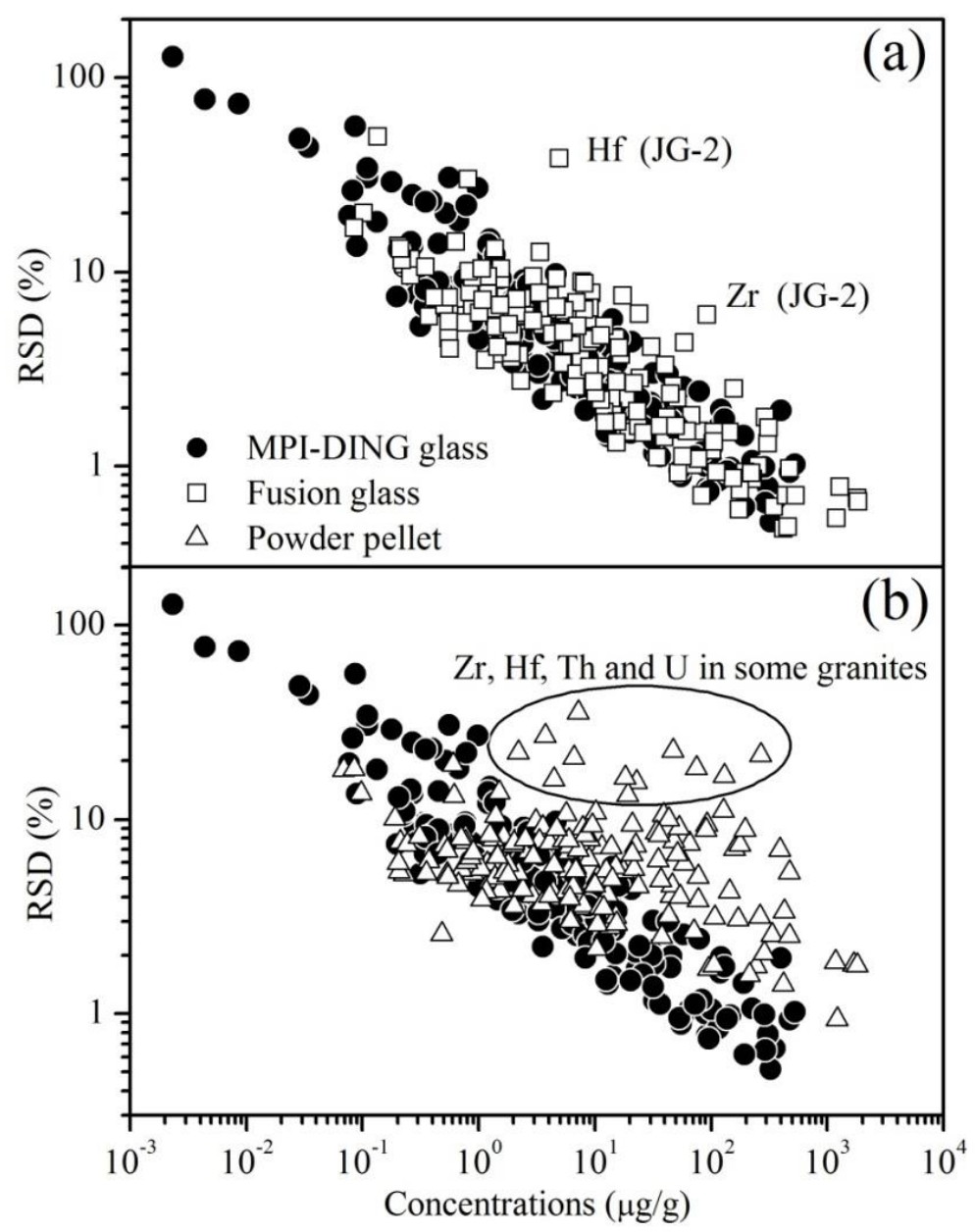

Fig.10 LA-ICP-MS analytical precisions of (a) flux-free fusion glass and (b) ultrafine powder pellet. The analytical precisions are given as one RSD $(n=10)$. Granitic samples include GSP-2, G-2, GBW07103, GBWO7111, JG-2 and JG-3. Elements plotted here are included Sc, V, Co, Rb, Sr, Y, Zr, Nb, Cs, Ba, La, Ce, Pr, Nd, Sm, Eu, Gd, Tb, Dy, Ho, Er, Tm, Yb, Lu, Hf, Ta, Th and U. 
Chapter 3.1 Comparison of Ultrafine Powder Pellet and Flux-free Fusion Glass for Bulk Analysis of Granitic Rock Samples by Laser Ablation-Inductively Coupled Plasma-Mass Spectrometry

Figure 10.a reveals that the analytical precisions of fusion glasses produced with our technique are similar to that of MPI-DING glasses, except for $\mathrm{Hf}$ and $\mathrm{Zr}$ in JG-2. The relative large RSD of Hf and $\mathrm{Zr}$ in JG-2 are probably caused by their heterogeneous distribution. Due to the higher $\mathrm{SiO}_{2}$ content $(76.8 \% \mathrm{~m} / \mathrm{m})$ in $\mathrm{JG}-2$, Hf and $\mathrm{Zr}$ concentrations were not homogenized as in the other glasses, even with $4 \mathrm{~h}$ melting at $1600{ }^{\circ} \mathrm{C}$ and two times of grinding. In contrast, the analytical precision of ultrafine powder pellets is generally worse compared to fusion glasses. The relatively larger RSD for ultrafine powder pellets are probably ascribed to the remaining larger crystal fragments (up to $15 \mu \mathrm{m}$ ). This interpretation is also supported with transient signals generated during the laser ablation (Appendix S4). Previous results (Garbe-Schönberg and Müller 2014) revealed that the analytical precision of ultrafine powder pellets from basaltic rocks is in a range of $0.1-5 \%$, which is better than that from granitic rocks (1-15\%). Figure 10.b shows that $\mathrm{Zr}$, Hf, Th, and $\mathrm{U}$ in some granites have large RSD (10-30\%). These results are comparable to the data (Hf: 17\%, Th: 16\% in GA) in the ultrafine powder pellets for the GA reference material (Garbe-Schönberg and Müller 2014). Several elements have even relatively larger RSD (>15\%), however, in our study, most of the elements with the content range from 0.1 to $1000 \mu \mathrm{g} / \mathrm{g}$ have RSD below $10 \%$.

\subsubsection{Evaluation of analytical accuracy}

\subsubsection{Calibration with NIST610, GSD-1G, and StHs6/80-G}

A large number of publications covering trace element determination with LA-ICP-MS demonstrated that the non-matrix matched calibration is the crucial factor influencing the analytical accuracy. Liu et al. (2008) illustrated that USGS glasses as calibration materials are better than NIST glass for the quantitative analysis of MPI-DING glasses. Garbe-Schönberg and Müller (2014) indicated that after initial non-matrix matched calibration with NIST glasses, a second calibration step with matrix-matched standards is obligatory to obtain accurate results for powder pellets. Our ablation behavior data also suggest that there is a matrix effect in the ablation process (Fig. 8, 9). Thus, the analytical accuracy of powder pellets and fusion glasses obtained from different calibration standards (including NIST610, GSD-1G, and StHs6/80-G) needs to be evaluated (Fig.11).

The downhole fractionation induced by laser ablation is one of the factors affecting the LA-ICP-MS accuracy. In our study, a relatively low laser energy density (around $5 \mathrm{~J} / \mathrm{cm}^{-2}$ ) and short ablation time 
Chapter 3.1 Comparison of Ultrafine Powder Pellet and Flux-free Fusion Glass for Bulk Analysis of Granitic Rock Samples by Laser Ablation-Inductively Coupled Plasma-Mass Spectrometry

(35 s) were used to keep the ratio between ablation depth and spot size less than one. Under these conditions, the down-hole fractionations in reference glasses, fusion glasses, and powder pellets are insignificant. Results of most elements calibrated with NIST610, GSD-1G, and StHs6/80-G are within analytical precision. The slight difference can be ascribed to the uncertainties of certified values. Therefore any matrix effects from the ablation process are effectively corrected with a known internal standard. However, for elements with extremely low concentrations (for example Mg, K in NIST 610) or some elements with large uncertainty (Mo in StHs6/80-G) in calibrated reference materials, the data calibrated with this reference material results in unreliable values for those elements.

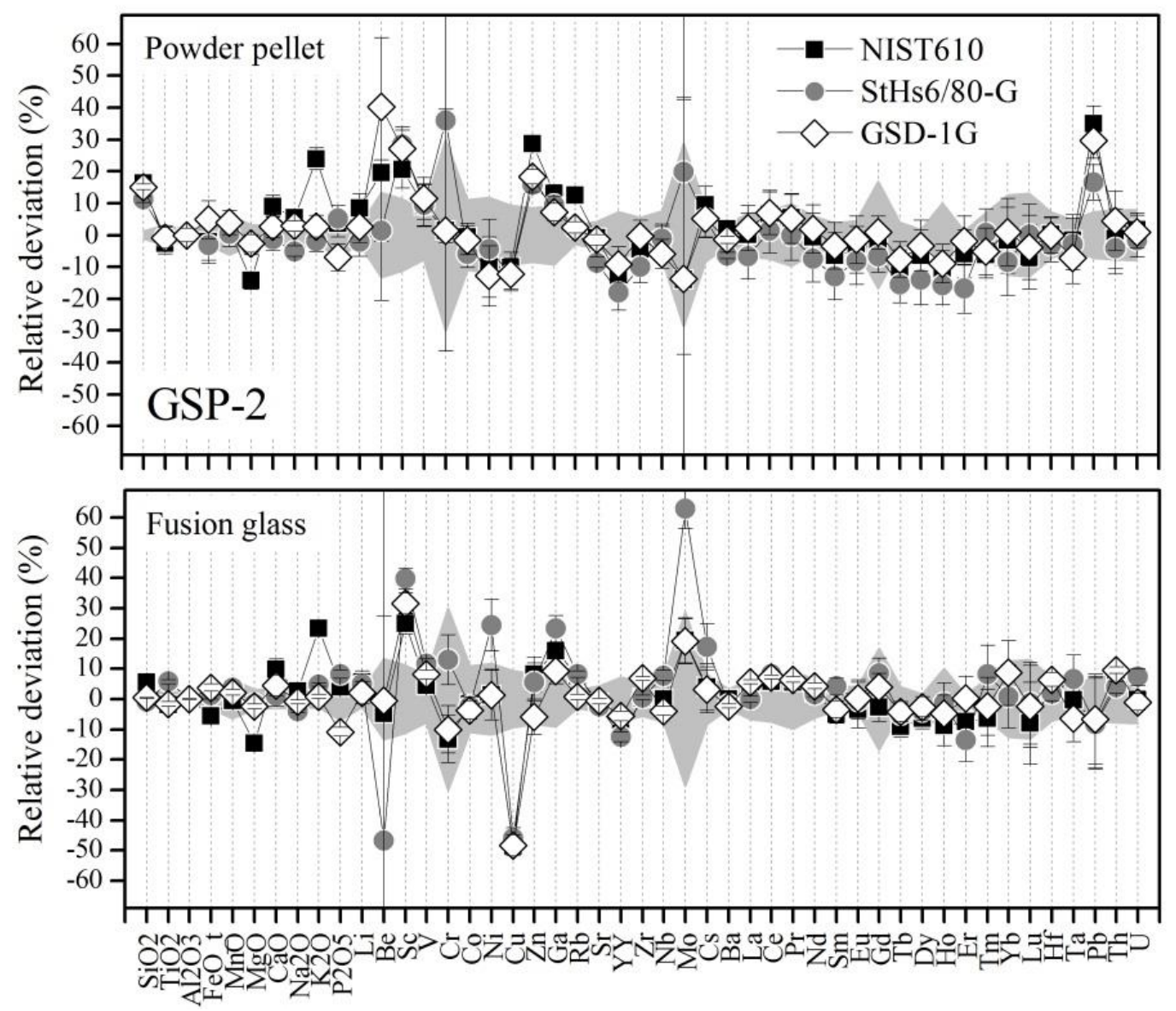

Fig.11 Evaluation of analytical accuracy obtained from three calibration materials (NIST610, StHs6/80-G and GSD-1G) for powder pellet and fusion glasses. The sample shown here is GSP-2. The grey zone represents the uncertainty of certified values (95\% confidence). Error bars are derived from eight repeated single analysis. 
Chapter 3.1 Comparison of Ultrafine Powder Pellet and Flux-free Fusion Glass for Bulk Analysis of Granitic Rock Samples by Laser Ablation-Inductively Coupled Plasma-Mass Spectrometry

4.5.2 Evaluation of LA-ICP-MS data from powder pellet and fusion glass

To avoid the potential unreliable values for some elements with single calibration material, the LA-ICP-MS data are compiled from the dataset obtained from NIST610, StHs6/80-G, and GSD-1G as external reference materials. The analytical data calibrated with an extremely low content element in reference standards were excluded from the data complication. The results are shown in Table 3 and Fig.12. Figure 12 shows that the data of granite samples obtained from powder pellets and fusion glass are similar, and generally matched well with reference values within $95 \%$ confidence, except for JG-2, which demonstrates that the LA-ICP-MS accuracies obtained from ultrafine powder pellets and the fusion glasses are comparable. The significant difference of data in JG-2 between powder pellets and fusion glass may be ascribed to the heterogeneity of original powders. Systematically lower values of Gd, Tb, and Dy for both ultrafine power pellet and fusion glass were observed in G-2. Considering this phenomenon only occurred in G-2, we conclude that the reference values of Gd, Tb, and Dy are probably imprecise. In summary, the LA-ICP-MS data obtained either from powder pellets or fusion glasses matched well with certified values within reference uncertainty. This demonstrates that our optimized techniques (ultrafine powder pellets and fusion glasses) are applicable for chemical analysis of granitic samples by LA-ICP-MS.

\Fig.12 Evaluation of LA-ICP-MS data obtained from ultrafine powder pellet and flux-free fusion glass. The LA-ICP-MS data are compiled from the dataset of NIST610, StHs6/80-G and GSD-1G as calibration materials. The grey zone represents the uncertainty of certified values (95\% confidence). The error bars are derived from different number repeated single analysis $(n=8-76)$ 

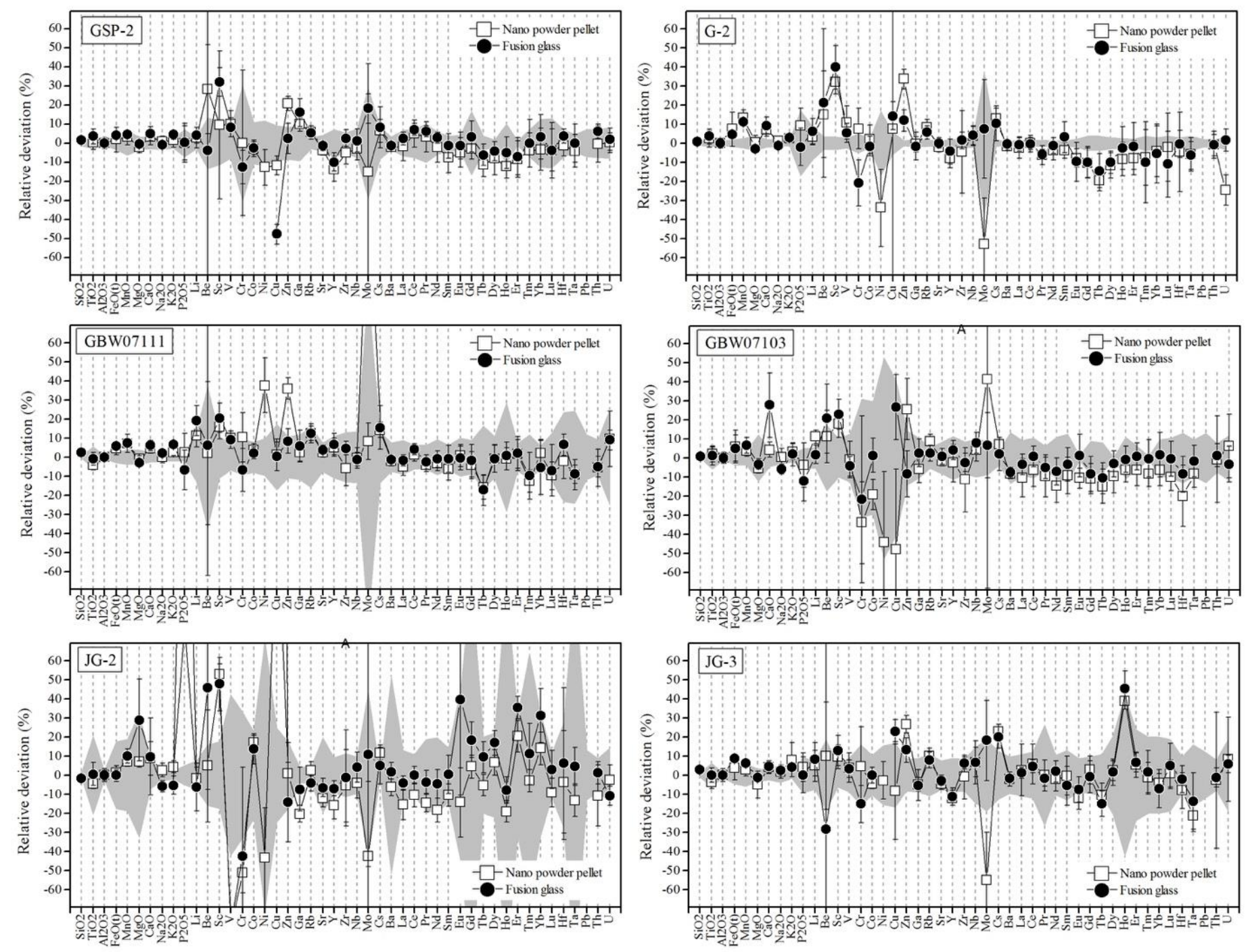


\subsubsection{Conclusions}

The wet-milling parameters specified to granitic samples for generating ultrafine powders was optimized. The ultrafine powders particles were characterized as $\mathrm{d}_{50}=1.2 \mu \mathrm{m}, \mathrm{d}_{50}=5.5$ $\mu \mathrm{m}$. Contaminations mainly stem from two sources: agate abrasion and the addition of ultrapure water. The results illustrate that the abrasion of agate balls was around 2-3\% relative to the original powder mass, however, the chemical composition is "clean" to rock powders.

A flux-free fusion protocol (fusion-grinding-fusion) was proposed to produce homogeneous glasses from granitic samples. The results illustrate that the re-grinding step is essential to obtain homogeneous glass. Highly volatile elements (e.g. $\mathrm{Pb}$ ) were depleted during fusion. Our procedure for making homogeneous glasses $\left(1600{ }^{\circ} \mathrm{C}, 2 \mathrm{~h}\right.$ melting, 15 min re-grinding) represents a compromise, as the glass is homogeneous enough for representative sampling by LA-ICP-MS, and at the same time, the highly volatile elements are not significantly depleted.

Laser ablation rates of ultrafine powder pellets and flux-free fusion glasses were investigated. The data reveal that laser ablation rates are specified for individual substrates (reference glasses, fusion glasses as well as powder pellets), which demonstrate that the matrix effects exist in laser ablation. Analytical precision of fusion glasses is comparable to MPI-DING glasses, while the precision of powder pellets is slightly worse, which might be ascribed to the remaining larger crystal fragments. This interpretation is supported by the less stability of the LA-ICP-MS transient signals. Analytical accuracy of both ultrafine powder pellets and flux-free fusion glasses is approvingly acceptable. The preparation methods by using ultrafine powder pellets and fusion glass are practical for bulk analysis of granitic samples with LA-ICP-MS. 


\section{Acknowledgments}

We thank Dr. Gerald Hartmann for the help in preparing powder pellet and to Dr. Andreas Kronz for the supervision of EPMA elemental mapping and SEM imaging. Financial support by the China Scholarship Committee (Nr. 201306410007) is greatly acknowledged.

\section{References}

Eggins S.M. (2003) Laser ablation ICP - MS analysis of geological materials prepared as lithium borate glasses Geostandards and Geoanalytical Research, 27, 147-162.

Eggins S.M., Kinsley L.P.J. and Shelley J.M.G. (1998) Deposition and element fractionation processes during atmospheric pressure laser sampling for analysis by ICP-MS Applied Surface Science, 127-129, 278-286.

Fedorowich J., Richards J., Jain J., Kerrich R. and Fan J. (1993) A rapid method for REE and trace element analysis using laser sampling ICP-MS on direct fusion whole-rock glasses Chemical Geology, 106, 229-249.

Frost B.R., Barnes C.G., Collins W.J., Arculus R.J., Ellis D.J. and Frost C.D. (2001) A geochemical classification for granitic rocks Journal of Petrology, 42, 2033-2048.

Gao S., Liu X.M., Yuan H.L., Hattendorf B., Günther D., Chen L. and Hu S.H. (2002) Determination of Forty Two Major and Trace Elements in USGS and NIST SRM Glasses by Laser Ablation-Inductively Coupled Plasma-Mass Spectrometry Geostandards Newsletter, 26, 181-196. 
Garbe-Schönberg D. and Müller S. (2014) Nano-particulate pressed powder tablets for LA-ICP-MS Journal of Analytical Atomic Spectrometry, 29 990-1000.

Gray A.L. (1985) Solid sample introduction by laser ablation for inductively coupled plasma source mass spectrometry Analyst, 110, 551-556.

He Z., Huang F., Yu H., Xiao Y., Wang F., Li Q., Xia Y. and Zhang X. (2016) A Flux-Free Fusion Technique for Rapid Determination of Major and Trace Elements in Silicate Rocks by LA-ICP-MS Geostandards and Geoanalytical Research, 40, 5-21.

Horn I., Guillong M. and Günther D. (2001) Wavelength dependent ablation rates for metals and silicate glasses using homogenized laser beam profiles - implications for LA-ICP-MS Applied Surface Science, 182, 91-102.

Hu M.Y., Fan X.T., Stoll B., Kuzmin D., Liu Y., Liu Y.S., Sun W.D., Wang G., Zhan X.C. and Jochum K.P. (2011a) Preliminary Characterisation of New Reference Materials for Microanalysis: Chinese Geological Standard Glasses CGSG-1, CGSG-2, CGSG-4 and CGSG-5 Geostandards and Geoanalytical Research, 35, 235-251.

Hu Z., Gao S., Liu Y., Xu J., Hu S. and Chen H. (2008) Niobium and tantalum concentrations in NIST SRM 610 revisited Geostandards and Geoanalytical Research, 32, 347-360.

Hu Z., Liu Y., Li M., Gao S. and Zhao L. (2009) Results for Rarely Determined Elements in MPI - DING, USGS and NIST SRM Glasses Using Laser Ablation ICP - MS Geostandards and Geoanalytical Research, 33, 319-335.

Hu Z.C., Liu Y.S., Chen L., Zhou L.A., Li M., Zong K.Q., Zhu L.Y. and Gao S. (2011b) Contrasting matrix induced elemental fractionation in NIST SRM and rock glasses during laser ablation ICP-MS analysis at high spatial resolution Journal of Analytical Atomic 
Spectrometry, 26, 425-430.

Imai N., TERASHIMA S., ITOH S. and ANDO A. (1995) 1994 compilation of analytical data for minor and trace elements in seventeen GSJ geochemical reference samples,"Igneous rock series" Geostandards and Geoanalytical Research, 19, 135-213.

Jarvis K.E. and Williams J.G. (1993) Laser ablation inductively coupled plasma mass spectrometry (LA-ICP-MS): a rapid technique for the direct, quantitative determination of major, trace and rare-earth elements in geological samples Chemical Geology, 106, 251-262.

Jochum K.P., Dingwell D.B., Rocholl A., Stoll B., Hofmann A.W., Becker S., Besmehn A., Bessette D., Dietze H.J. and Dulski P. (2000)

The Preparation and Preliminary Characterisation of Eight Geological MPI - DING Reference Glasses for In - Situ Microanalysis Geostandards Newsletter, 24, 87-133.

Jochum K.P., Stoll B., Herwig K., Willbold M., Hofmann A.W., Amini M., Aarburg S., Abouchami W., Hellebrand E., Mocek B., Raczek I., Stracke A., Alard O., Bouman C., Becker S., Dücking M., Brätz H., Klemd R., de Bruin D., Canil D., Cornell D., de Hoog C.-J., Dalpé C., Danyushevsky L., Eisenhauer A., Gao Y., Snow J.E., Groschopf N., Günther D., Latkoczy C., Guillong M., Hauri E.H., Höfer H.E., Lahaye Y., Horz K., Jacob D.E., Kasemann S.A., Kent A.J.R., Ludwig T., Zack T., Mason P.R.D., Meixner A., Rosner M., Misawa K., Nash B.P., Pfänder J., Premo W.R., Sun W.D., Tiepolo M., Vannucci R., Vennemann T., Wayne D. and Woodhead J.D. (2006) MPI-DING reference glasses for in situ microanalysis: New reference values for element concentrations and isotope ratios Geochemistry, Geophysics, Geosystems, 7, 1-44.

Jochum K.P., Weis U., Schwager B., Stoll B., Wilson S.A., Haug G.H., Andreae M.O. and Enzweiler J. (2016) Reference values following ISO guidelines for frequently requested rock reference materials Geostandards and Geoanalytical Research, 40(3), 333-350. 
Jochum K.P., Weis U., Stoll B., Kuzmin D., Yang Q.C., Raczek I., Jacob D.E., Stracke A.,

Birbaum K., Frick D.A., Günther D. and Enzweiler J. (2011) Determination of Reference

Values for NIST SRM 610-617 Glasses Following ISO Guidelines Geostandards and Geoanalytical Research, 35, 397-429.

Johnson D., Hooper P. and Conrey R. (1999) XRF analysis of rocks and minerals for major and trace elements on a single low dilution Li-tetraborate fused bead. Advances in X-ray Analysis, 41, 843-867.

Klemm W. and Bombach G. (2001) A simple method of target preparation for the bulk analysis of powder samples by laser ablation inductively coupled plasma mass spectrometry (LA-ICP-MS) Fresenius' Journal of Analytical Chemistry, 370, 641-646.

Koch J. and Günther D. (2011) Review of the State-of-the-Art of Laser Ablation Inductively Coupled Plasma Mass Spectrometry Applied Spectroscopy, 65, 155A-162A.

Kurosawa M., Shima K., Ishii S. and Sasa K. (2006) Trace Element Analysis of Fused Whole - Rock Glasses by Laser Ablation-ICP-MS and PIXE Geostandards and Geoanalytical Research, 30, 17-30.

Liu Y.S., Hu Z.C., Gao S., Gunther D., Xu J., Gao C.G. and Chen H.H. (2008) In situ analysis of major and trace elements of anhydrous minerals by LA-ICP-MS without applying an internal standard Chemical Geology, 257, 34-43.

Liu Y.S., Hu Z.C., Li M. and Gao S. (2013) Applications of LA-ICP-MS in the elemental analyses of geological samples Chinese Science Bulletin, 58, 3863-3878.

Müller W., Shelley M., Miller P. and Broude S. (2009) Initial performance metrics of a new 
Chapter 3.1 Comparison of Ultrafine Powder Pellet and Flux-free Fusion Glass for Bulk Analysis of Granitic Rock Samples by Laser Ablation-Inductively Coupled Plasma-Mass Spectrometry

custom-designed ArF excimer LA-ICPMS system coupled to a two-volume laser-ablation cell Journal of Analytical Atomic Spectrometry, 24, 209-214.

Mukherjee P.K., Khanna P.P. and Saini N.K. (2014) Rapid Determination of Trace and Ultra Trace Level Elements in Diverse Silicate Rocks in Pressed Powder Pellet Targets by LA-ICP-MS using a Matrix-Independent Protocol Geostandards and Geoanalytical Research, $38,363-379$.

Norman M., Pearson N., Sharma A. and Griffin W. (1996) Quantitative analysis of trace elements in geological materials by laser ablation ICPMS: instrumental operating conditions and calibration values of NIST glasses Geostandards Newsletter, 20, 247-261.

Park C.-S., Shin H.S., Oh H., Cho H. and Cheong A.C.-s. (2016) Trace element analysis of whole-rock glass beads of geological reference materials by Nd: YAG UV $213 \mathrm{~nm}$ LA-ICP-MS Journal of Analytical Science and Technology, 7, 1-8.

Paton C., Hellstrom J., Paul B., Woodhead J. and Hergt J. (2011) Iolite: Freeware for the visualisation and processing of mass spectrometric data Journal of Analytical Atomic Spectrometry, 26, 2508-2518.

Pearce J.A., Harris N.B. and Tindle A.G. (1984) Trace element discrimination diagrams for the tectonic interpretation of granitic rocks Journal of Petrology, 25, 956-983.

Peters D. and Pettke T. (2017) Evaluation of Major to Ultra Trace Element Bulk Rock Chemical Analysis of Nanoparticulate Pressed Powder Pellets by LA - ICP - MS Geostandards and Geoanalytical Research, 41, 5-28.

Pretorius W., Weis D., Williams G., Hanano D., Kieffer B. and Scoates J. (2006) Complete 
Trace Elemental Characterisation of Granitoid (USGS G-2, GSP-2) Reference Materials by High Resolution Inductively Coupled Plasma - Mass Spectrometry Geostandards and Geoanalytical Research, 30, 39-54.

Raczek I., Stoll B., Hofmann A.W. and Peter Jochum K. (2001) High-Precision Trace Element Data for the USGS Reference Materials BCR-1, BCR-2, BHVO-1, BHVO-2, AGV-1, AGV-2, DTS-1, DTS-2, GSP-1 and GSP-2 by ID-TIMS and MIC-SSMS Geostandards and Geoanalytical Research, 25, 77-86.

Reid J.E., Horn I., Longerich H.P., Forsythe L. and Jenner G.A. (1999) Determination of Zr and Hf in a Flux-Free Fusion of Whole Rock Samples using Laser Ablation-Inductively Coupled Plasma-Mass Spectrometry (LA-ICP-MS) with Isotope Dilution Calibration Geostandards and Geoanalytical Research, 23, 149-155.

Russo R.E., Mao X.L., Liu H.C., Gonzalez J. and Mao S.S. (2002) Laser ablation in analytical chemistry - a review Talanta, 57, 425-451.

Stoll B., Jochum K.P., Herwig K., Amini M., Flanz M., Kreuzburg B., Kuzmin D., Willbold M. and Enzweiler J. (2008) An automated iridium-strip heater for LA-ICP-MS bulk analysis of geological samples Geostandards and Geoanalytical Research, 32, 5-26.

Sylvester P. (2001) Trace element analysis of fused whole rock glasses by laser ablation ICPMS Laser-Ablation-ICPMS in the Earth Sciences: Principles and Applications. Mineralogical Association of Canada Short Course Series, 29, 147-162.

Sylvester P.J. and Jackson S.E. (2016) A Brief History of Laser Ablation Inductively Coupled Plasma Mass Spectrometry (LA-ICP-MS) Elements, 12, 307-310.

Tabersky D., Luechinger N.A., Rossier M., Reusser E., Hametner K., Aeschlimann B., Frick 
D.A., Halim S.C., Thompson J., Danyushevsky L. and Gunther D. (2014) Development and characterization of custom-engineered and compacted nanoparticles as calibration materials for quantification using LA-ICP-MS Journal of Analytical Atomic Spectrometry, 29(6), 955-962.

Tamura A., Akizawa N., Otsuka R., Kanayama K., Python M., Morishita T. and Arai S. (2015) Measurement of whole-rock trace-element composition by flux-free fused glass and LA-ICP-MS: evaluation of simple and rapid routine work Geochemical Journal, 49, 243-258.

Wasson J.T. (1985) Meteorites: their record of early solar-system history. W. H. Freeman and Co., New York, NY, 274pp.

Whalen J.B., Currie K.L. and Chappell B.W. (1987) A-type granites: geochemical characteristics, discrimination, and petrogenesis Contributions to Mineralogy and Petrology, 95, 407-419.

Wilson S. (1998) Data compilation for USGS reference material GSP-2, Granodiorite, Silver Plume, Colorado US Geological Survey Open-File Report, 11-12.

Wu S., Wang Y., Zhan X., Kronz A., Simon K., Xu C. and Tian H. (2016) Study on the Elemental Fractionation Effect of CGSG Reference Materials and the Related Within-Unit Homogeneity of Major and Trace Elements Rock and Mineral Analysis, 35, 612-620.

Yang Q.C., Jochum K.P., Stoll B., Weis U., Kuzmin D., Wiedenbeck M., Traub H. and Andreae M.O. (2012) BAM-S005 Type A and B: New Silicate Reference Glasses for Microanalysis Geostandards and Geoanalytical Research, 36, 301-313.

Yu Z., Norman M.D. and Robinson P. (2003) Major and Trace Element Analysis of Silicate Rocks by XRF and Laser Ablation ICP-MS Using Lithium Borate Fused Glasses: Matrix 
Chapter 3.1 Comparison of Ultrafine Powder Pellet and Flux-free Fusion Glass for Bulk Analysis of Granitic Rock Samples by Laser Ablation-Inductively Coupled Plasma-Mass Spectrometry

Effects, Instrument Response and Results for International Reference Materials Geostandards and Geoanalytical Research, 27, 67-89.

Yu Z., Robinson P. and McGoldrick P. (2001) An Evaluation of Methods for the Chemical Decomposition of Geological Materials for Trace Element Determination using ICP - MS Geostandards and Geoanalytical Research, 25, 199-217.

Yu Z., Robinson P., Townsend A.T., Mnker C. and Crawford A.J. (2000) Determination of High Field Strength Elements, Rb, Sr, Mo, Sb, Cs, Tl and Bi at ng g- 1 Levels in Geological Reference Materials by Magnetic Sector ICP-MS after HF/HClO4 High-Pressure Digestion Geostandards and Geoanalytical Research, 24, 39-50.

Zhang C., Hu Z., Zhang W., Liu Y., Zong K., Li M., Chen H. and Hu S. (2016) A green and fast laser fusion technique for bulk silicate rock analysis by laser ablation ICP-MS Analytical Chemistry, 88, 10088-10094.

Zhu L.Y., Liu Y.S., Hu Z.C., Hu Q.H., Tong X.R., Zong K.Q., Chen H.H. and Gao S. (2013) Simultaneous Determination of Major and Trace Elements in Fused Volcanic Rock Powders Using a Hermetic Vessel Heater and LA-ICP-MS Geostandards and Geoanalytical Research, 37, 207-229. 
Chapter 3.2 Signal enhancement in LA-ICP-MS analysis by guard

electrode and the addition of nitrogen and hydrogen into carrier gas:

\section{A perspective from experiment}

Shitou Wu*, Klaus Simon

Geowissenschaftliches Zentrum, Göttingen Universität, Göttingen 37077, Germany

Corresponding author E-mail address: wushitou111@hotmail.com

Fax: +49 $55139-23982$ 
Abstract: The signal enhancement effects using guard electrode and the addition of small amount nitrogen $\left(\mathrm{N}_{2}\right)$ and hydrogen $\left(\mathrm{H}_{2}\right)$ into the carrier gas flow $(\mathrm{Ar}+\mathrm{He})$ of the Ar plasma in laser ablation inductively coupled plasma mass spectrometry (LA-ICP-MS) is presented. The results illustrate that signal intensity of 54 investigated elements is enhanced up to 6 folds by using guard electrode compared to without guard electrode, which is related to an increase in the ion density that was induced by the shrinkage of the whole plasma due to the guard electrode. Guard electrode shifts the ionization zone backward to sample cone that needs a larger carrier gas flow to compensate. A small amount of $\mathrm{H}_{2}$ decreases the signal sensitivity in GE-off mode (with guard electrode), while slightly enhances the sensitivity in GE-on mode (without guard electrode). A small amount of $\mathrm{N}_{2}$ shifts the ionization zone backward to the sample cone in both of GE-off and GE-on modes. The results illustrate that those six modes (GE-off, GE-off-N $\mathrm{N}_{2}$, GE-off- $\mathrm{H}_{2}$, GE-on, GE-on-N 2 , GE-on- $\mathrm{H}_{2}$ ) produced very similar analytical data. The GE-on- $\mathrm{N}_{2}$ with $\left(2 \mathrm{ml} \mathrm{min}^{-1}\right)$ is the best instrument conditions for routine multiple trace element analysis.

Keywords: Laser ablation-inductively coupled plasma-mass spectrometry; Signal enhancement; Guard electrode; Molecular gas 


\subsubsection{Introduction}

Laser ablation-inductively coupled plasma-mass spectrometry (LA-ICP-MS) as a prominent in situ technique has been widely used in analytical geochemistry (Koch and Günther, 2011; Liu et al., 2013; Russo et al., 2013). Its applications cover trace element (Gagnon et al., 2008; Garbe-Schönberg and Müller, 2014; Liu et al., 2008; Stoll et al., 2008) and isotopic ratio determination (Kaiyun et al., 2014; Tong et al., 2015) in various samples (glass, mineral, fluid/melted inclusion, etc.) for diverse purposes, which include 2D/3D elemental mapping (Raimondo et al., 2017; Ubide et al., 2015), depth profile analysis (Mank and Mason, 1999), geochronology (Guillong et al., 2016; Jackson et al., 2004; Spandler et al., 2016; Yuan et al., 2004; Zack and Hogmalm, 2016; Zack et al., 2011) as well as geothermometer research (Cruz - Uribe et al., 2016; Flem et al., 2002). In recent years, with the requirement of elemental analysis at high spatial resolution $(<10 \mu \mathrm{m})$ by LA-ICP-MS (Macholdt et al., 2015; Petrelli et al., 2016; Tomlinson et al., 2010; Wu et al., 2016), signal enhancement for achieving greater sensitivity is drawing more attention. Signal enhancement in LA-ICP-MS analysis mainly involves four aspects, (1) addition of small amount molecular gas (e.g., $\mathrm{H}_{2}$, $\mathrm{N}_{2}$, and $\mathrm{O}_{2}$ ) into carrier gas (Durrant, 1994; M. Guillong and Heinrich, 2007; ZC Hu et al., 2008; Kosler et al., 2014); (2) modification of sample and skimmer cones (Latkoczy and Günther, 2002; Xu et al., 2015); (3) improving vacuum system (Kimura et al., 2013) and (4) using guard electrode (Appelblad et al., 2000; Tong et al.,, 2015). Among these approaches, the addition of small amount molecular gas into carrier gas and using guard electrode are commonly accepted due to their simplicity and feasibility.

Signal enhancement by the addition of small amount molecular gas (e.g. $\mathrm{N}_{2}, \mathrm{H}_{2}$, and $\mathrm{O}_{2}$ ) into plasma has been proved in previous studies. Small amount $\mathrm{N}_{2}$ added into carrier gas could improve the energy transfer from the plasma towards the injected species, and small amount $\mathrm{H}_{2}$ added into carrier gas could lead to a higher electron density in plasma. Durrant (1994) investigated the effect of $\mathrm{N}_{2}$ in argon plasma and demonstrated that the introduction of small amount $\mathrm{N}_{2}$ increased the signal sensitivity and consequently reduced oxide to metal ratios $\left(\mathrm{MO}^{+} / \mathrm{M}^{+}\right)$. A similar phenomenon has been reported by Hu et al. (2008), who illustrated that 
the signal enhancement is related to the better energy transfer of $\mathrm{Ar}-\mathrm{N}_{2}$ plasma. However, other studies showed opposite phenomena. Tong et al. (2015) reported that no significant sensitivity enhancement was achieved for $\mathrm{Sr}$ by bleeding $\mathrm{N}_{2}$ into the plasma. Fu et al. (2016) investigated the addition of $\mathrm{N}_{2}$ on the performance of in situ $\mathrm{S}$ isotope analyses and demonstrated that the signal intensity of $\mathrm{S}$ was not increased with the addition of $\mathrm{N}_{2}$, but the related polyatomic interferences of $\mathrm{OO}, \mathrm{SH}, \mathrm{OOH}$ were significantly reduced, and the mass bias stability zone has been enlarged. The enhancement effect of the addition of $\mathrm{H}_{2}$ into plasma has also been debated in previous studies. Guillong and Heinrich (2007) reported that the addition of 4-9 $\mathrm{ml} \mathrm{min}^{-1}$ of $\mathrm{H}_{2}$ to the helium carrier gas flow increased the sensitivity for most of 47 investigated elements by a factor of 2-4, while Durrant (1993) indicated that little benefit had been found with the addition of $\mathrm{H}_{2}$ in either solution nebulization ICP-MS or LA-ICP-MS. Those observations demonstrated that the signal enhancement with the addition of $\mathrm{N}_{2}$ or $\mathrm{H}_{2}$ into plasma might be influenced by the difference of ICP-MS setups, which include torch design, sampling interface, etc. Previous studies are mainly focused on quadruple ICP-MS, and little data has been reported with the sector field ICP-MS, which is nowadays widely installed in LA-ICP-MS laboratory.

Guard electrode is inserted between the torch and load coil to diminish the plasma offset potential. The offset potential between plasma and sample cone may lead a serious secondary ion discharge effect and increase kinetic energy spread. Compared to the addition of a small amount of molecular gas for signal enhancement, the guard electrode is convenient and does not introduce any extra matrix, but it is related to stronger polyatomic ion interferences (Tong et al., 2015) and oxide formation (Appelblad et al., 2000). Appelblad et al. (2000) systematically investigated the performance characteristics of the guard electrode in solution nebulization ICP-MS and reported that using the guard electrode (grounded torch) could lead to a signal enhancement, but with more oxide formations and severe non-spectral interference matrix effects. Becker and Dietze (1999) reported that the usage of guard electrode yielded an increase in sensitivity up to a factor of 5 with Meinhard, MicroMist microconcentric, and ultrasonic nebulizers. Chen et al. (2015) documented that the sensitivity of 39 investigated 
isotopes was increased by a factor of 17 58 using a guard electrode and both $\mathrm{CeO}^{+} / \mathrm{Ce}^{+}$and $\mathrm{Ce}^{2+} / \mathrm{Ce}^{+}$ratios were suppressed with the addition of $4 \%$ ethanol. Guard electrode has merits including diminished plasma offset potentials, thus reducing kinetic energy spread and leading to higher ion transmission efficiency. Xu et al. (2015) illustrated that the influence of a guard electrode on the sensitivity of $\mathrm{Sr}$ was minimal, but became significant in the presence of $\mathrm{N}_{2}$. These previous studies mainly focused on solution nebulization ICP-MS. However, a systematic study of signal enhancement of a guard electrode in LA-ICP-MS is still lacking.

In this study, we investigated the effects of the addition of $\mathrm{N}_{2}$ and $\mathrm{H}_{2}$ in combination with the guard electrode on the signal intensities of elements from ${ }^{7} \mathrm{Li}$ to ${ }^{238} \mathrm{U}$, oxide yields and doubly charged ion yields in LA-ICP-MS. To gain insight into the mechanisms of signal intensity change, the spatial profiles of ICP ion distribution were made in the presence or absence of $\mathrm{N}_{2}$ and $\mathrm{H}_{2}$ in combination with the guard electrode. The analytical accuracy and precision at different modes based on six glass reference materials were evaluated.

\subsubsection{Experiment}

\subsubsection{Samples and reagents}

To investigating the analytical accuracy and precision, glass reference materials including NIST610, StHs6/80-G, GSD-1G, BCR-2G, KL2-G, and GOR132-G were used. These glasses are mostly acceptable as LA-ICP-MS calibration materials and well characterized in previous studies (Jochum et al., 2000; Jochum et al., 2006; Jochum et al., 2011; Pearce et al., 1997). The reference values and their uncertainty are cited from literature (Jochum et al., 2006; Jochum et al., 2011) and GeoReM database (Jochum et al., 2005) (http://georem.mpch -mainz.gwdg.de/). Hydrogen ( $\mathrm{H}_{2}$, purity 5.0, Air Products Company), nitrogen ( $\mathrm{N}_{2}$, purity 5.0, Linde Company) were employed as addition gases. The additional gas flow is controlled by a mass flow controller with a range up to $20 \mathrm{ml} \mathrm{min}^{-1}$. 


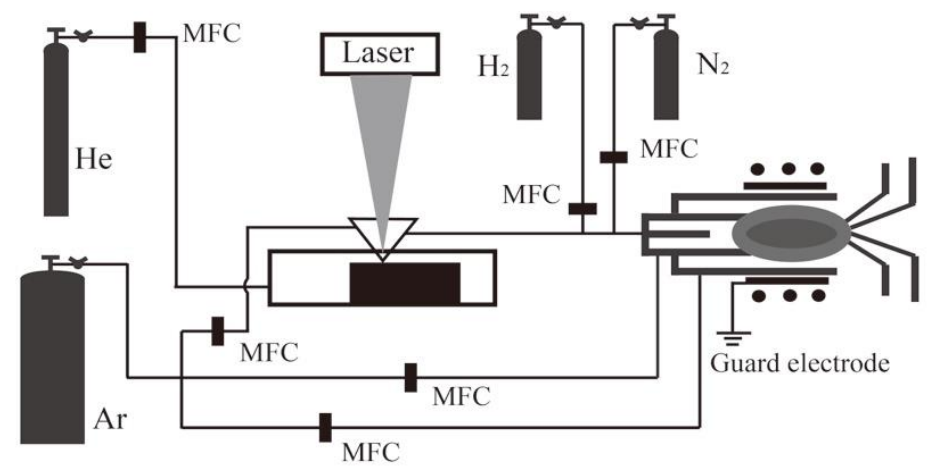

Fig. 1 Schematic set-up of the laser ablation ICP-MS system for the addition of small amounts of nitrogen and hydrogen. MFC denotes mass flow controller.

Table1 LA-ICP-MS instrumentation

\section{Laser ablation system}

Model \& type

Ablation cell \& volume

Laser wavelength

Pulse width

Energy density/fluence

Repetition rate

Laser ablation system

Spot size

Ablation rate

Sampling mode/pattern

Ablation gas flow (He)

Addition gas

Ablation duration

\section{ICP-MS Instrument}

Model \& type

RF Power

Guard electrode

Sample cone

Skimmer cone

Coolant gas flow (Ar)

Auxiliary gas flow (Ar)

Make-up gas flow (Ar)

Scan mode

Segment duration

Detection system

Resolution $(\mathrm{M} / \Delta \mathrm{M})$
Resonetics resolution M-50

Laurin Technics 155, aerosol dispersion volume $<1 \mathrm{~cm}^{3}$

$193 \mathrm{~nm}$

$20 \mathrm{~ns}$

$\sim 3 \mathrm{~J} \mathrm{~cm}^{-2}$

$5 \mathrm{~Hz}$

$50 \mu \mathrm{m}$

$\sim 120 \mathrm{~nm}$ per pulse for NIST glass, $\sim 80 \mathrm{~nm}$ per pulse for MPI-DING

Single hole drilling, two cleaning pulses

$0.00 \sim 1.00 \mathrm{l} / \mathrm{min}$

$\mathrm{H}_{2}$ and $\mathrm{N}_{2}$ in a range of $0.0 \sim 5.0 \mathrm{ml} / \mathrm{min}$

20 seconds

Thermo Element 2 SF-ICP-MS

$1400 \mathrm{~W}$

Floated and grounded $(\mathrm{Pt})$

Ni 54605

Ni $543540.8 \mathrm{H}$

$15.00 \mathrm{l} / \mathrm{min}$

$1.00 \mathrm{l} / \mathrm{min}$

$0.30 \sim 1.15 \mathrm{l} / \mathrm{min}$ (mixed with He inside ablation cell funnel)

E-scan

$10 \mathrm{~ms}$ for all scanned isotopes

Single detector double mode SEM, counting, analog

Low (around 300)

$1 \mathrm{~s}$ 


\subsubsection{Instrumentation}

Experiments were carried out using an Element 2 sector field ICP-MS instrument (Thermo Scientific, USA) coupled to a $193 \mathrm{~nm}$ ArF excimer laser ablation system (RESOlution M-50, Australian Scientific Instruments, Australia). Helium (He) was used as ablation environment gas. Detailed information of the ablation cell and schematic gas connection have been described in the literature (Müller et al., 2009). Some important instrumental parameters are summarized in Table 1.

\subsubsection{Results and discussion}

\subsubsection{Optimization of Ar and $\mathrm{He}$}

Argon (Ar) was first selected as the carrier gas to carry the ablation aerosol into ICP. Later Eggins et al. (1998) and Günther and Heinrich (1999) demonstrated that the sensitivity was improved when Helium (He) was used as carrier gas. He is a lighter gas compared to Ar. Thus it could enhance transport of ablation aerosols to the ICP, thereby increases the signal sensitivity. However, the relatively large first ionization potential of $\mathrm{He}(\mathrm{He}: 24.587 \mathrm{eV}$, Ar: $15.759 \mathrm{eV}$ ) could result in a decrease of electron density and temperature in plasma. Here, we conducted an experiment with changing both He and Ar gas flow rates to understand their influence on the plasma. The experiment was carried out at $1400 \mathrm{~W}$ power and without using guard electrode and without the addition of $\mathrm{N}_{2}$ and $\mathrm{H}_{2} \cdot{ }^{88} \mathrm{Sr}$ were used for signal monitoring and ${ }^{88} \mathrm{Sr}$ background, $\mathrm{ThO} / \mathrm{Th}$, and $\mathrm{U} / \mathrm{Th}$ were used for the evaluation of plasma conditions.

The results show that the sum of He and Ar gas flow rate is a crucial factor influencing the signal intensity (Fig.2), which illustrates that a sufficient gas flow rate needs to push the ionization zone to the optimum position towards sample cone. Figure 2 shows that around $100 \mathrm{ml} \mathrm{min}{ }^{-1} \mathrm{He}$ flow rate is enough to carry almost all ablation aerosols. He flow rate seems more sensitive compared to Ar, especially when the gas flow of He exceed $600 \mathrm{ml} \mathrm{min}^{-1}$. That is supposedly related to a reduction in electron density and cooling of the plasma due to the higher ionization potential of He. Figure 2 illustrates that the background intensity of $\mathrm{Sr}$ is related to the sum of $\mathrm{He}$ and Ar gas flow. The higher He and Ar gas flow lead to a lower 
background intensity. The $\mathrm{ThO} / \mathrm{Th}$ ratio is increased with the increase of He and Ar flow rate. That is ascribed to the cooling effects of the plasma. The Higher gas flow could reduce the plasma temperature, and thus increase the oxide production rates. U/Th ratio is used for monitoring the plasma conditions. $\mathrm{U}$ and Th have similar physicochemical properties, and the concentrations of U and Th in NIST610 are almost identical. The higher gas flow resulted in a plasma condition with more ionized $\mathrm{U}$ than $\mathrm{Th}$.

The gas flow of He in the range of 350 to $650 \mathrm{ml} \mathrm{min}-1$ and Ar in the range of 800 to $950 \mathrm{ml}$ min-1 represents the optimum gas conditions, in which a high signal intensity is achieved with low background signal and $\mathrm{ThO} / \mathrm{Th}$ (lower than $0.1 \%$ ), and with the $\mathrm{U} / \mathrm{Th}$ kept in the range of 0.95 to 1.05 .
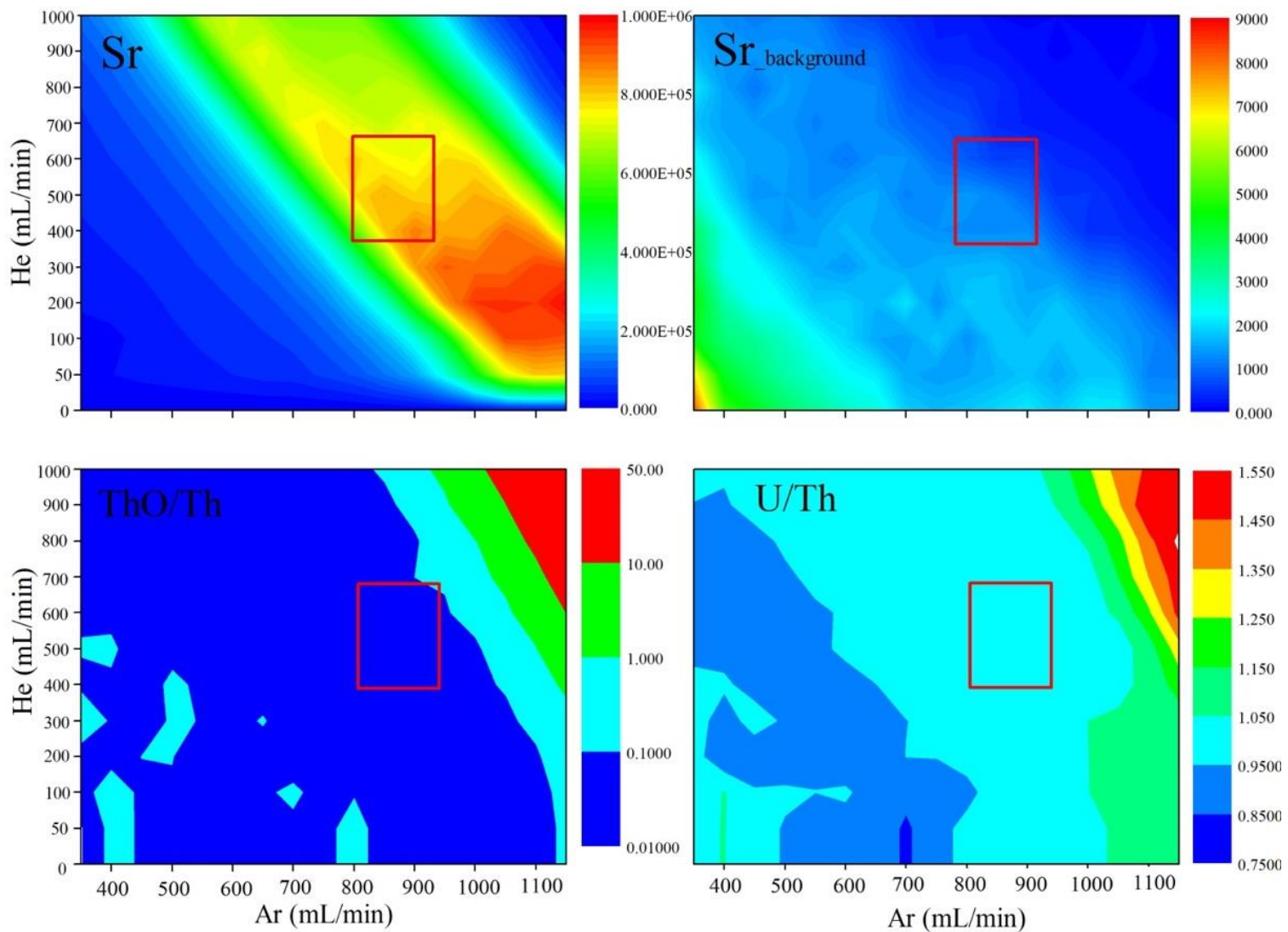

Fig.2 plasma conditions with the change of $\mathrm{He}$ and Ar gas flow rate by monitoring Sr signal intensity, $\mathrm{Sr}$ background intensity, $\mathrm{ThO} / \mathrm{Th}$ and $\mathrm{U} / \mathrm{Th}$. The experiment was carried out at 1400W power and without using guard electrode and without the addition of N2 and H2. The solid line box marked with red color represents the optimal gas flow rates. 


\subsubsection{Effect on sensitivity}

In previous studies, guard electrode has been proved to lead to a signal enhancement (Appelblad et al., 2000; Tong et al., 2015). Here, the Sr signal intensity and its background were used as monitors to investigate the effect of guard electrode. Ar flow rate was optimized at a range of $500 \sim 1150 \mathrm{ml} \mathrm{min}{ }^{-1}$ on the condition that He flow was fixed at $500 \mathrm{ml} \mathrm{min}^{-1}$ in GE-off (without using guard electrode) and GE-on (with using guard electrode) modes. Results are shown in Fig.3. A fixed He gas flow to make the ablation aerosol into ICP as constant. Figure 3 illustrates that guard electrode shifted the ionization zone backward to sample cone which needs a larger carrier gas flow to compensate. The signal sensitivity has been enhanced around four folders in GE-on mode; however, the corresponding background also increased. This demonstrates that the signal enhancement of guard electrode is probably related to the shrinkage of the whole plasma.
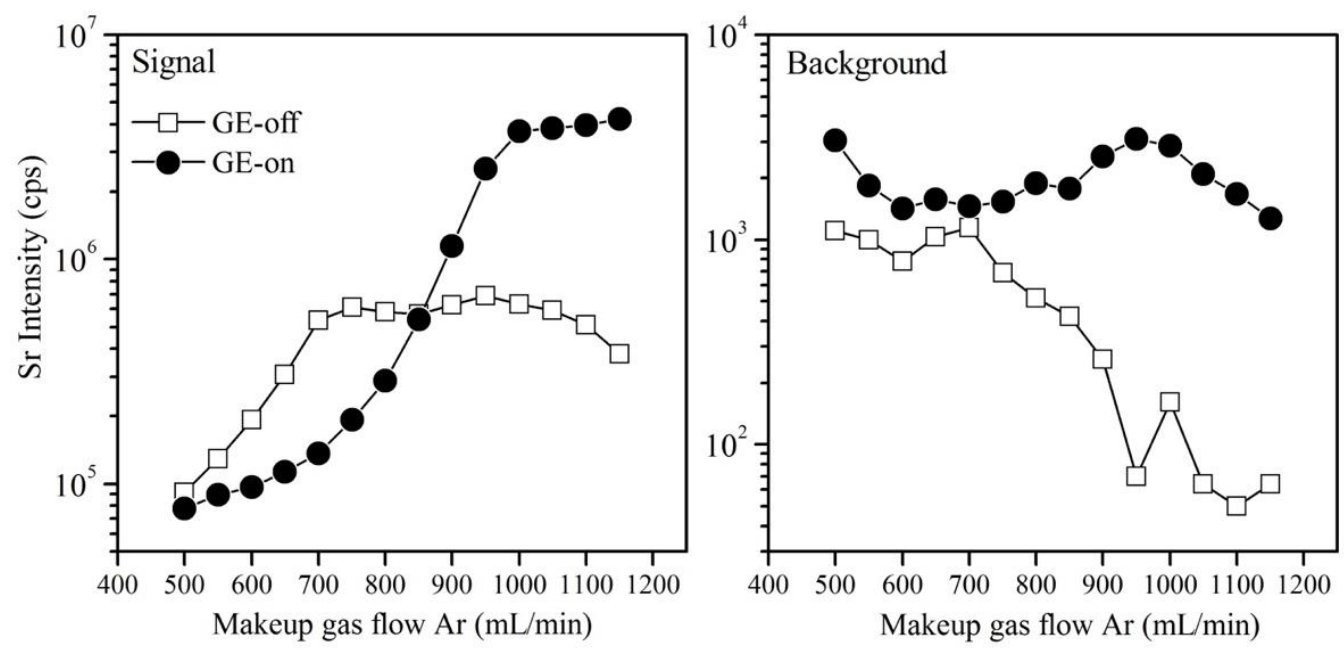

Fig.3 Variations of Sr signal and background intensity with the change of Ar gas flow rate in a range of $500 \sim 1150 \mathrm{ml} \mathrm{min}{ }^{-1}$. He gas flow rate is fixed as $500 \mathrm{ml} \mathrm{min}^{-1}$

The influence of the addition of $\mathrm{N}_{2}$ and $\mathrm{H}_{2}$ in combination with guard electrode was investigated. Figure 4 shows the intensity of $\mathrm{La}$ at the experiment conditions of no $\mathrm{N}_{2}$ and $\mathrm{H}_{2}$,

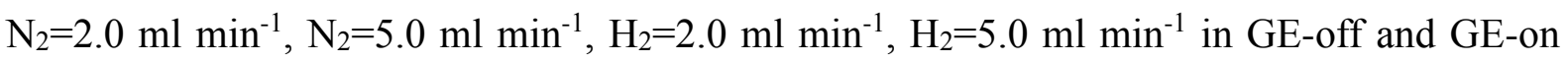
modes. The results illustrated the addition of $\mathrm{H}_{2}$ decreased the signal sensitivity in GE-off mode, while slightly enhanced the sensitivity in GE-on mode. The degree of reduction is 
negatively correlated to the amount of $\mathrm{H}_{2}$. This observation is inconsistent with what Guillong and Heinrich (2007) reported. To further know the mechanism, we plotted the suppression factor of background and signal intensity of $\mathrm{Na}, \mathrm{Ti}$, and $\mathrm{Mn}$. The results are shown in Fig.5. The results illustrate that the suppression factor of background is larger than that of intensity. The mechanism behind this is unclear and needs further studies. A small amount of $\mathrm{N}_{2}$ shifted the ionization zone backward to the sample cone in GE-on and GE-off modes. A slight enhancement is found in GE-off mode, while no significant enhancement is observed in GE-on mode. The platform is wider with the addition of $\mathrm{N}_{2}$. To further investigate the signal enhancement in different modes concerning all elements, we plotted the signal enhancement of six modes (Fig.5). Figure 5 illustrates that GE-on with the addition of $\mathrm{N}_{2}$ is the best conditions, which have a 5-7 folders enhancement for all elements.
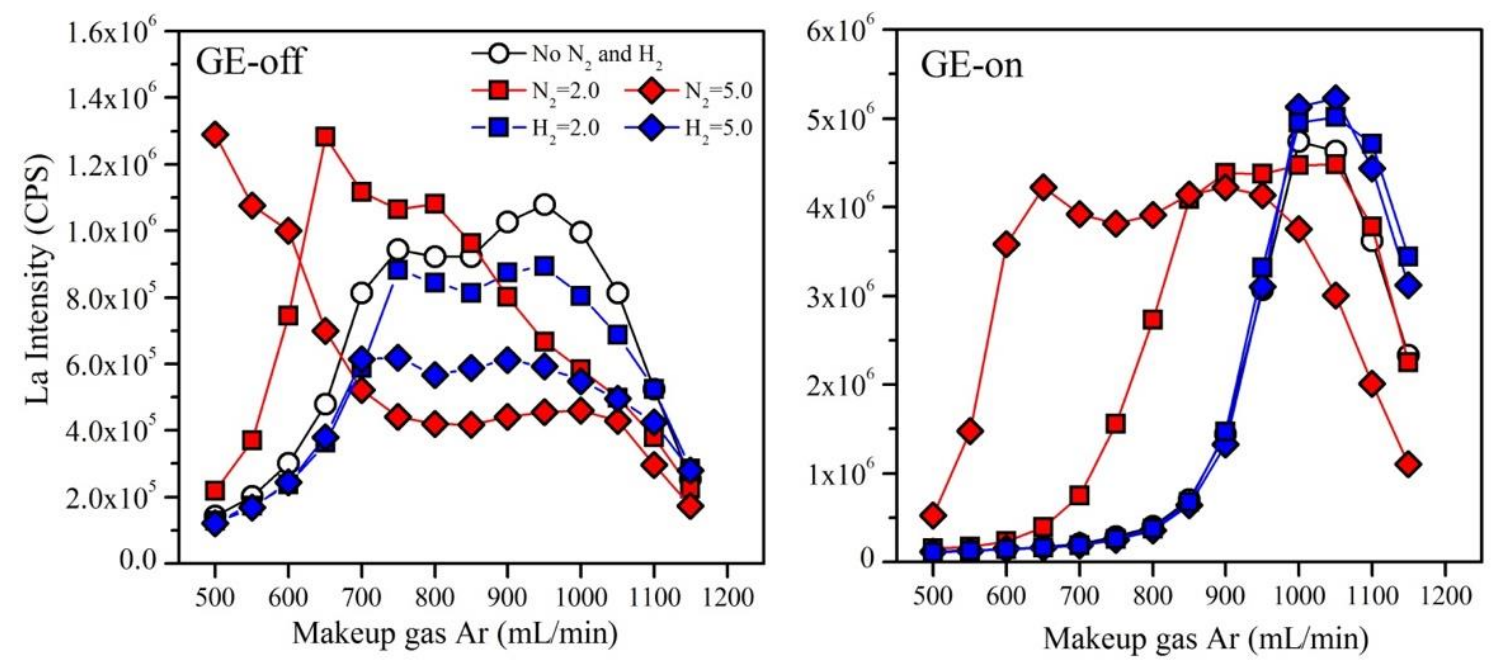

Fig.4 Signal intensity of La as a function of makeup gas (central channel gas) flow rate in the presence of absence of $\mathrm{N}_{2}$ and $\mathrm{H}_{2}$ with and without the guard electrode. The addition of $\mathrm{H}_{2}$

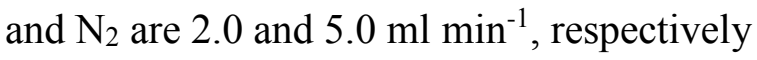




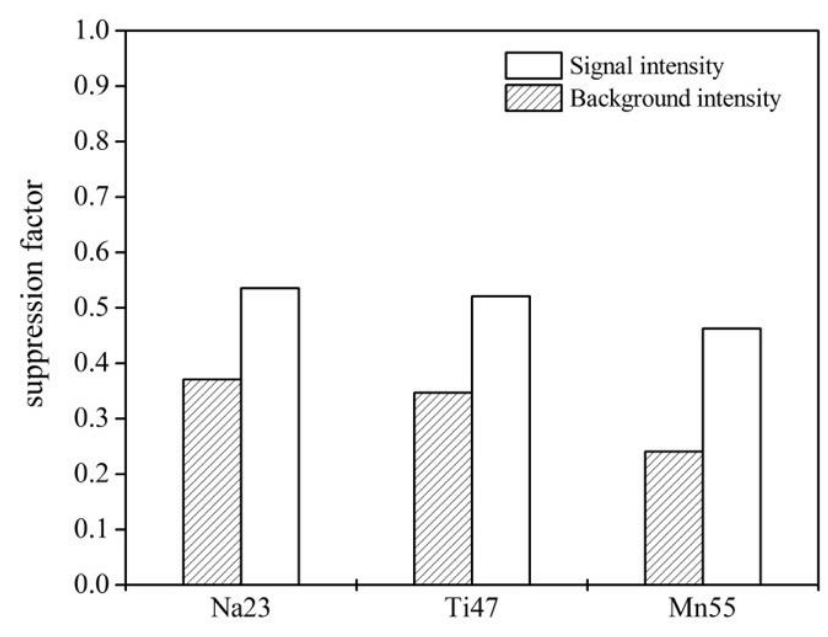

Fig.5 Suppression factors of background and signal intensity of $\mathrm{Na}$, Ti, and $\mathrm{Mn}$ in GE-off- $\mathrm{H}_{2}$ modes

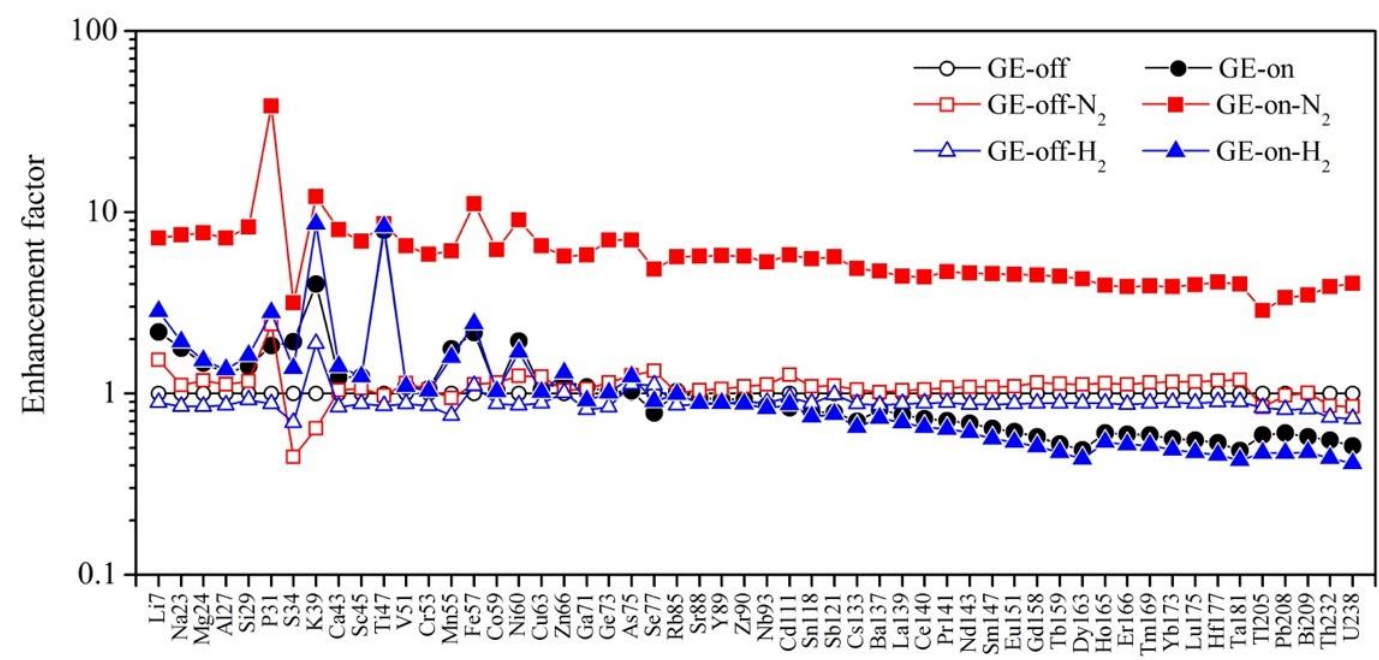

Fig.6 Sensitivity enhancement factors of 54 elements in six modes including GE-off, GE-off- $\mathrm{H}_{2}$, GE-off- $\mathrm{N}_{2}$, GE-off, GE-off- $\mathrm{H}_{2}$, and GE-off- $\mathrm{N}_{2}$. The enhancement factors are calculated by dividing the intensity from other five modes to the GE-off mode

\subsubsection{Effects on oxide and doubly charged ions}

Spectral interferences caused by oxides (such as ${ }^{135} \mathrm{Ba}^{16} \mathrm{O}->{ }^{151} \mathrm{Eu}$ ) and doubly charged ions (such as ${ }^{138} \mathrm{Ba}^{2+}-^{69} \mathrm{Ga}^{+}$) are of particular importance for LA-ICP-MS and frequently affect the analytical accuracy. ${ }^{232} \mathrm{Th}$ and ${ }^{43} \mathrm{Ca}$ were selected for the investigation of oxide (ThO/Th), and doubly charged ion $\left(\mathrm{Ca}^{2+} / \mathrm{Ca}^{+}\right)$yields. Results are shown in Fig.6 and Fig.7. All signals were corrected for the gas background. 

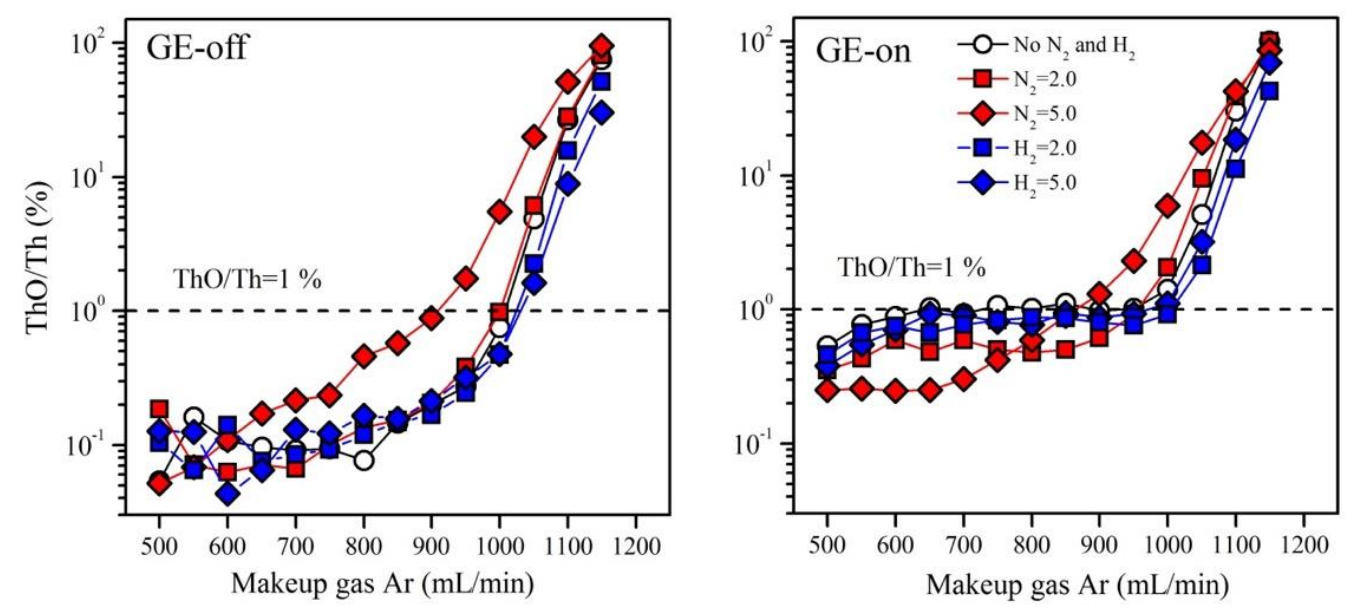

Fig.7 Effect of makeup gas flow rate on $\mathrm{ThO}^{+} / \mathrm{Th}^{+}$ratio at $\mathrm{rf}$ powers of $1400 \mathrm{~W}$ for the GE-off and GE-on modes with and without the addition of $\mathrm{N}_{2}$ and $\mathrm{H}_{2}$. The dashed lines show the $\mathrm{ThO}^{+} / \mathrm{Th}^{+}$ratio equal to $1 \%$.

As shown in Fig.7, the oxide production rate increases with the growth of carrier gas flow rate in GE-on and GE-off modes with the addition of $\mathrm{N}_{2}$ and $\mathrm{H}_{2}$, which is consistent with previous observations (Liu et al., 2014; Nonose et al., 1994; Segura et al., 2003). Oxide production rate grew slower with Ar gas flow less than $1000 \mathrm{ml} \mathrm{min}^{-1}$, while it grew rapidly when the carrier gas flow rate increase to $1000 \mathrm{ml} \mathrm{min}$. This phenomenon could be explained by the cooling effects of plasma with the increase of gas flow rate. In general, oxide yields in GE-on mode are greater than that of the GE-off mode in the addition of $\mathrm{N}_{2}$ and $\mathrm{H}_{2}$, which means oxide formation in GE-on mode may be enhanced at the plasma-interface (Liu et al., 2014) due to the shrinkage effects. Further studies need to be conducted to understand oxide formation in GE-on mode. Oxide yields should be observed with more attention when conducting the gas tuning in GE-on mode.

The addition of $\mathrm{N}_{2}$ increases the oxide yields in a GE-off mode in the range of Ar gas flow $500 \sim 1150 \mathrm{ml} \mathrm{min}^{-1}$. That does not support the interpretation that the addition of $\mathrm{N}_{2}$ improves the energy transfer effects of plasma, at least not in the GE-off mode. The effect of the addition of $\mathrm{N}_{2}$ in GE-on mode is different from that in GE-off mode. The oxide yield is suppressed with the addition of $\mathrm{N}_{2}$ at the Ar gas flow rate smaller than $900 \mathrm{ml} \mathrm{min}^{-1}$, while 
enhanced when the Ar gas flow rate increase to $900 \mathrm{ml} \mathrm{min}^{-1}$. This result is consistent with what $\mathrm{Hu}$ et al. reported (ZC Hu et al.,, 2008). The improvement of energy transfer effects of plasma with the addition of $\mathrm{N}_{2}$ could not explain the underlying mechanism, which needs a further investigation. The addition of $\mathrm{H}_{2}$ suppresses the oxide yields in both GE-off and GE-on modes, which is probably caused by the formation of $\mathrm{OH}^{+}$, which decreases other oxides production rates.
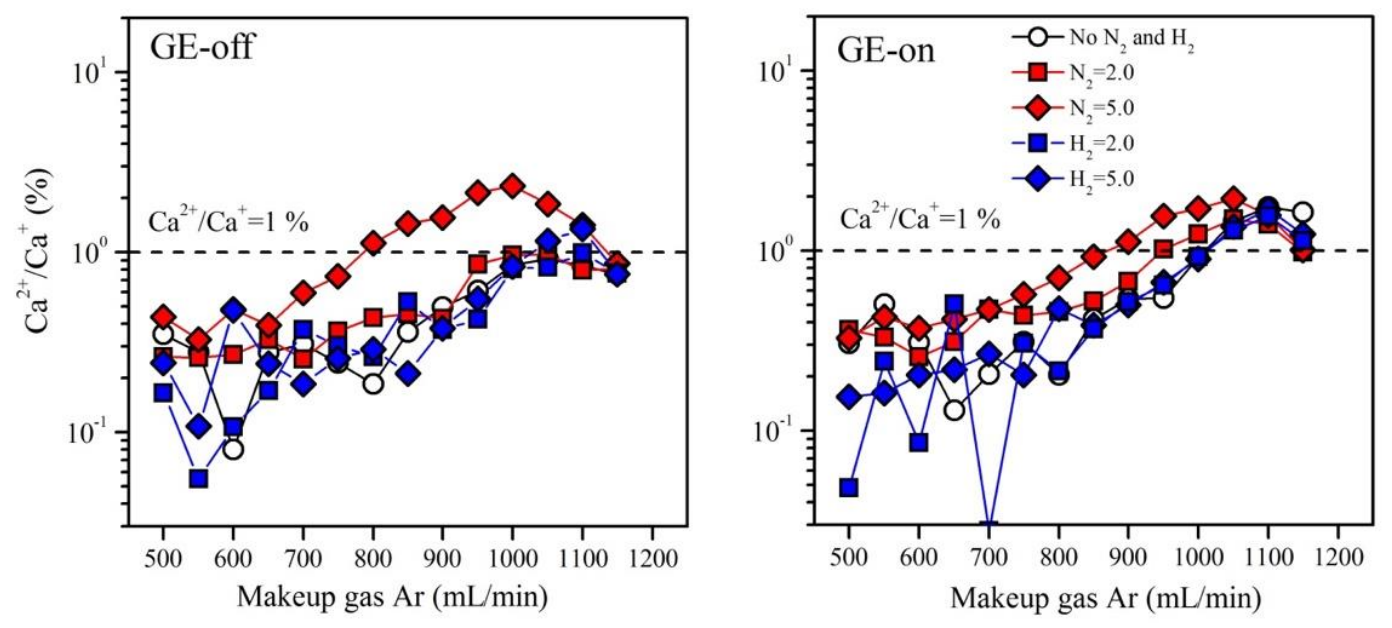

Fig.8 Effect of makeup gas flow rate on the $\mathrm{Ca}^{2+} / \mathrm{Ca}^{+}$ratio at $\mathrm{rf}$ powers of $1400 \mathrm{~W}$ for the GE-off and GE-on modes with and without the addition of $\mathrm{N}_{2}$ and $\mathrm{H}_{2}$. The dashed lines show the $\mathrm{Ca}^{2+} / \mathrm{Ca}^{+}$ratio equal to $1 \%$.

As demonstrated Fig.7, the $\mathrm{Ca}^{2+} / \mathrm{Ca}^{+}$ratio increased until reaching a plateau with the increase of makeup gas flow rate in GE-off and GE-on modes, while the $\mathrm{Ca}^{2+} / \mathrm{Ca}^{+}$ratio was slightly larger in GE-on compared with GE-off. Doubly charged ions are mostly formed by the secondary discharge (Sakata and Kawabata, 1994). Using guard electrode could reduce such secondary discharge effects. Thereby the formation of doubly charged ions may not be caused by the secondary discharge, at least not in a sector field ICP-MS. Alternatively, higher production of $\mathrm{Ca}^{2+} / \mathrm{Ca}^{+}$ratios and its relation to the makeup gas flow may be linked to the ICP conditions. The addition of $\mathrm{N}_{2}$ in general increases the doubly charged ions production in both GE-off and GE-on modes, while the addition of $\mathrm{H}_{2}$ almost does not influence the doubly charged ions production. Thus the doubly charged ions should be paid more attention when conducting the gas tuning in GE-on mode with the addition of $\mathrm{N}_{2}$. 
Chapter 3.2 Signal enhancement in LA-ICP-MS analysis by guard electrode and the addition of nitrogen and hydrogen into carrier gas: A perspective from experiment

\subsubsection{Spatial profile of the ion distributions}

ICP ion spatial profiling not only provides information on the energy and time needed to form a given ion but also provide valuable insight into the predominant ionization mechanism in the ICP (Holliday and Beauchemin, 2004).
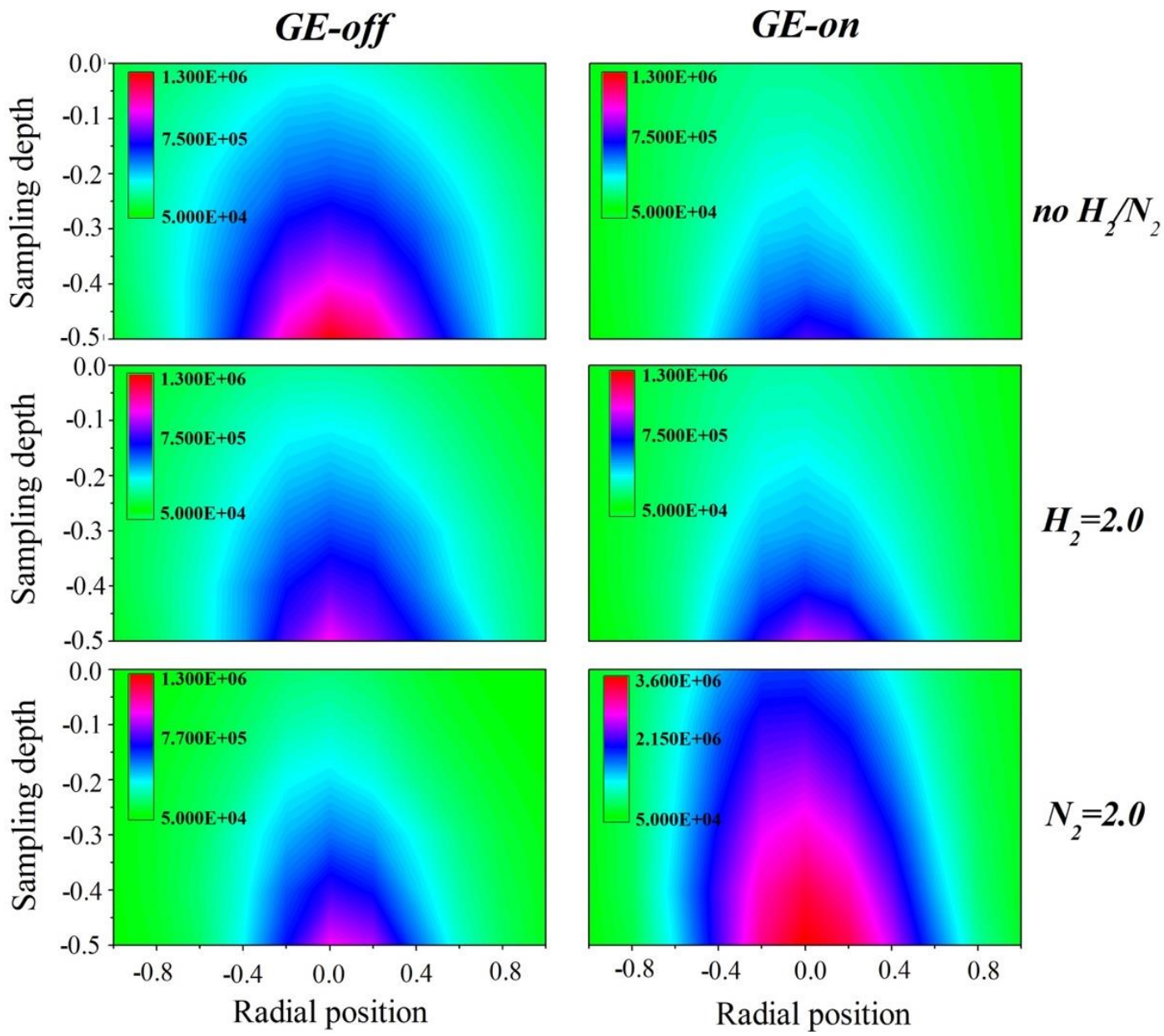

Fig.9 Radial and axial intensity distributions for La at $1400 \mathrm{~W}$ power, Ar gas flow $850 \mathrm{ml}$

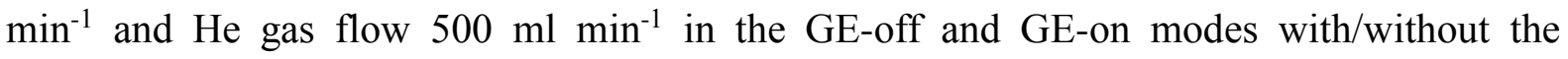
addition of $\mathrm{H}_{2}$ and $\mathrm{N}_{2}$. The addition of $\mathrm{H}_{2}$ and $\mathrm{N}_{2}$ are 2.0 and $5.0 \mathrm{ml} \mathrm{min}{ }^{-1}$, respectively.

The results illustrate that the guard electrode shifts the ionization zone backward to the sample cone. Therefore a larger carrier gas flow is needed for compensation. The shrinkage effect was observed in GE-on compared to GE-off mode without the addition of $\mathrm{N}_{2}$ and $\mathrm{H}_{2}$, which could be interpreted as the signal enhancement effect by using guard electrode. The axial ion distribution is expected to be a convolution between radial diffusion and 
vaporization of the aerosol particles following the flow of the carrier gas inside the central channel of the ICP. The guard electrode diminishes the plasma offset potential between plasma and sample cone, thus reducing the kinetic energy spread. The reduced ions kinetic energy spread would lead to a reduced ions diffusion in radial of plasma, thereby improve the ions transmission efficiency to sampling cone. This shrinkage effect will enhance the background intensity simultaneously, thus the signal enhancement effect by using guard electrode may not improve the signal/background ratio for these low mass element (having significant background intensity). The backgrounds of the high mass element are almost undetectable in both GE-off and GE-on modes, so that the signal enchantment is useful for the ultralow concentration high mass element in geological samples, especially for the improvement of precision.

The addition of $\mathrm{N}_{2}$ and $\mathrm{H}_{2}$ in GE-off mode leads to the shrinkage of ions distribution in plasma, while makes the enlarged ions distribution in GE-on mode, especially for the addition of $\mathrm{N}_{2}$. Xu et al. (2015) reported similar results and illustrated that the influence of a guard electrode on the sensitivity of Sr was minimal, but became significant in the presence of $\mathrm{N}_{2}$. These results demonstrate that the guard electrode plays a crucial role for the change of plasma with addition of $\mathrm{N}_{2}$ and $\mathrm{H}_{2}$, which needs a further investigation to understand the underlying mechanism.

\subsubsection{Effects on analytical accuracy}

The analytical accuracy of six modes (including GE-off, GE-off- $\mathrm{H}_{2}$, GE-off-N 2 , GE-off, GE-off- $\mathrm{H}_{2}$, and GE-off-N $\mathrm{N}_{2}$ ) is evaluated by analysis of StHs6/80-G, BCR-2G., and KL-2G. Results were calculated based on NIST610 as reference material and Ca as internal standard (Fig.10). The Relative Deviation (\%) is defined as [(elements $\left.)_{m}-(\text { elements })_{\text {ref }}\right] /(\text { elements })_{\text {ref }}$ $\times 100$. Fig. 10 illustrates that data obtained from six modes are substantially identical, except $\mathrm{K}$ and $\mathrm{P}$, which suffer $\mathrm{H}$ or $\mathrm{N}$ based interferences, and almost all elements match well with the reference values within $10 \%$ error at six modes. That demonstrates that the guard electrode and the addition of $\mathrm{N}_{2}$ and $\mathrm{H}_{2}$ are not affecting the analytical accuracy. It is worth 
Chapter 3.2 Signal enhancement in LA-ICP-MS analysis by guard electrode and the addition of nitrogen and hydrogen into carrier gas: A perspective from experiment

noting that results of major elements including $\mathrm{MgO}$ and $\mathrm{K}_{2} \mathrm{O}$ have a system deviation based on NIST610 as a calibration material for all sis modes. This is probably caused by the very low content of these two elements in NIST610, which leads to larger uncertainties when conducting single point calibration. This suggests that a certain matrix effect of major elements may exist between NIST610 and geological glasses.
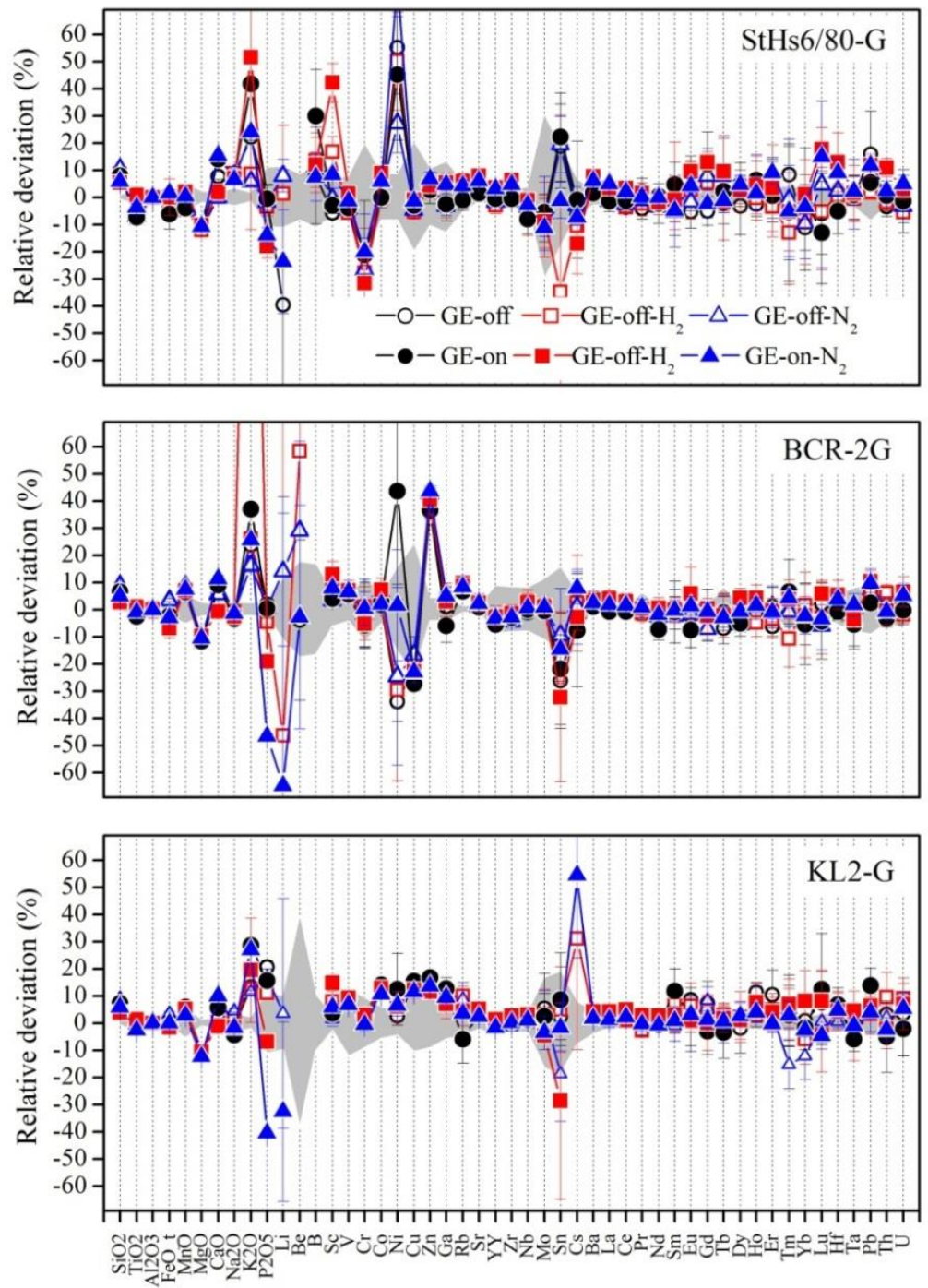

Fig.10 Comparison of reference values and the analytical results of StHs6/80-G, BCR-2G, and KL2-G obtained from six modes including GE-off, GE-off- $\mathrm{H}_{2}$, GE-off-N 2 , GE-off, GE-off- $\mathrm{H}_{2}$ and GE-off- $\mathrm{N}_{2}$

Analytical precision is an especially important factor for evaluating the quality of analytical data. Considering the significant enhancement at GE-on- $\mathrm{N}_{2}$ mode compared to GE-off mode, the analytical precision obtained from these two modes was assessed. The data have been 
Chapter 3.2 Signal enhancement in LA-ICP-MS analysis by guard electrode and the addition of nitrogen and hydrogen into carrier gas: A perspective from experiment

achieved by using NIST610 as reference materials and Ca as an internal standard. Multiple analysis ( $\mathrm{n}=5$ ) of MPI-DING reference materials (StHs6/80-G, ATHO-G, T1-G, ML3B-G, KL2-G, GOR132-G, and GOR128-G) was conducted. The laser spot size is $50 \mu \mathrm{m}$. Analytical precision is expressed as one time the relative standard deviation (\%RSD, $\mathrm{n}=12)$. Figure 11 shows the relationship between analytical precision and concentration in logarithmic scale for GE-on and GE-off modes.

Figure11 indicates that analytical precision is dominated by Poisson counting statistics uncertainty (Gao et al., 2002; $\mathrm{Hu}$ et al., 2009). And thus further demonstrates well homogeneity of the MPI-DING glass. A better improvement of analytical precisions by a factor of two was obtained at GE-on- $\mathrm{N}_{2}$ mode, which could be explained by the signal enhancement effects. Signal enhancement effects at GE-on- $\mathrm{N}_{2}$ mode lead to an improved sensitivity, thus reduces the uncertainty derived from Poisson counting statistics. Figure 11 shows that the analytical precision for all REEs at GE-on mode is less than $10 \%$ when the concentration is down to $0.1 \mu \mathrm{g} / \mathrm{g}$.

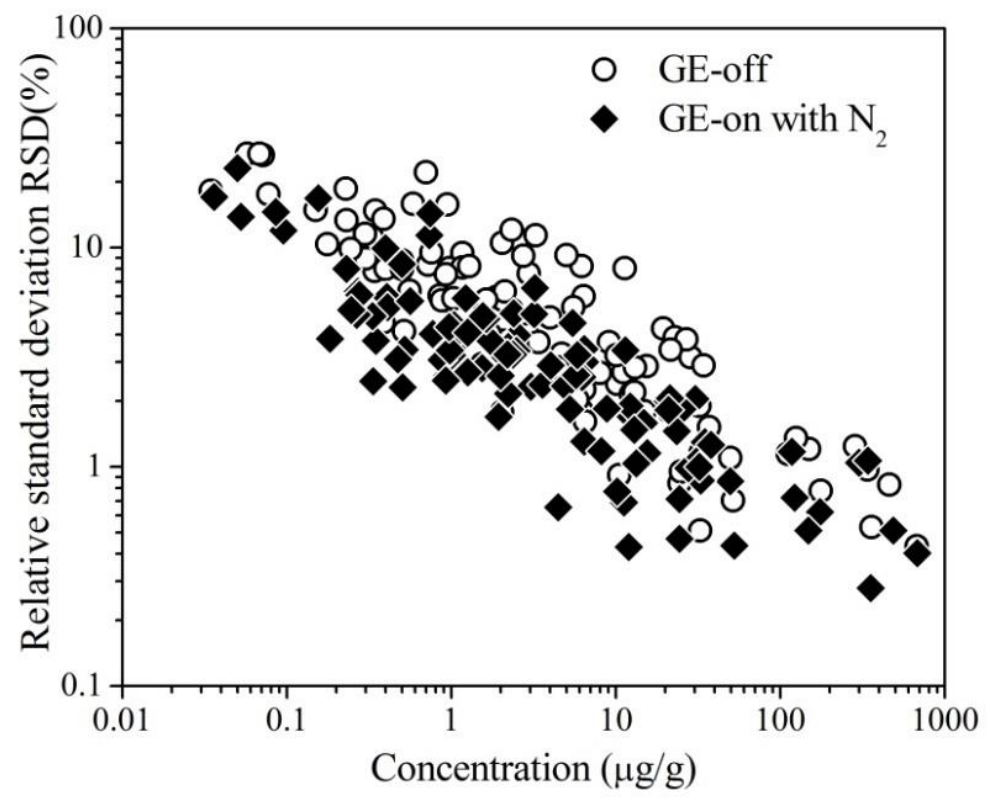

Fig.11 Concentrations versus RSD (\%) of the selected elements in MPI-DING glasses in GE-off and GE-on- $\mathrm{N}_{2}$ modes. The selected elements include $\mathrm{Sc}, \mathrm{Ga}, \mathrm{Rb}, \mathrm{Sr}, \mathrm{Y}, \mathrm{Zr}, \mathrm{Nb}, \mathrm{Cs}, \mathrm{Ba}$, La, Ce, Pr, Nd, Sm, Eu, Gd, Tb, Dy, Ho, Er, Tm, Yb, Lu, Hf, Ta, Th, U. The laser spot size is $50 \mu \mathrm{m}$. 


\subsubsection{Concluding remarks}

In this study, we investigated the signal enhancement effects by the addition of $\mathrm{N}_{2}$ and $\mathrm{H}_{2}$ in combination with the guard electrode on 54 elements from ${ }^{7} \mathrm{Li}$ to ${ }^{238} \mathrm{U}$, as well as oxide yields and doubly charged ion yields in LA-ICP-MS. The spatial profiles of ICP ion distribution are collected in the presence or absence of $\mathrm{N}_{2}$ and $\mathrm{H}_{2}$ in combination with the guard electrode. The analytical accuracy and precision at different modes based on six glass reference materials are evaluated. Several conclusions are made as follows,

1. Signal intensity of 54 investigated elements is enhanced up to 6 times by using guard electrode compared to without using guard electrode, which is related to the increase of ion density that is induced by the shrinkage of the whole plasma due to guard electrode.

2. Guard electrode shifts the ionization zone backward to sample cone that needs a larger carrier gas flow to compensate.

3. Small amounts of $\mathrm{H}_{2}$ decrease the signal sensitivity without using the guard electrode, while slightly enhance the sensitivity with the guard electrode.

4. Small amounts of $\mathrm{N}_{2}$ shift the ionization zone backward to the sample cone with and without the guard electrode.

5. Those six modes including GE-off, GE-off- $\mathrm{N}_{2}, \mathrm{GE}-$ off- $\mathrm{H}_{2}, \mathrm{GE}-\mathrm{on}, \mathrm{GE}-\mathrm{on}-\mathrm{N}_{2}, \mathrm{GE}-\mathrm{on}-\mathrm{H}_{2}$ ) produce very similar analytical data.

6. The GE-on- $\mathrm{N}_{2}\left(2 \mathrm{ml} \mathrm{min}{ }^{-1}\right)$ is the best instrument conditions for routine multiple trace element analysis. 


\section{References}

Appelblad PK, Rodushkin I, Baxter DC (2000) The use of Pt guard electrode in inductively coupled plasma sector field mass spectrometry: advantages and limitations. J. Anal. At. Spectrom. 15(4): 359-364

Becker JS, Dietze H-J (1999) Application of double-focusing sector field ICP mass spectrometry with shielded torch using different nebulizers for ultratrace and precise isotope analysis of long-lived radionuclides. J. Anal. At. Spectrom. 14(9): 1493-1500

Chen T, Hu Z, Liu S, Liu Y, Gao S, Li M, Zong K, Chen H, Hu S (2015) Improved performance of a shielded torch using ethanol in inductively coupled plasma-sector field mass spectrometry. Spectrochimica Acta Part B: Atomic Spectroscopy. 106: 36-44

Cruz-Uribe AM, Mertz-Kraus R, Zack T, Feineman MD, Woods G, Jacob DE (2016) A New LA-ICP-MS Method for Ti in Quartz: Implications and Application to High Pressure RutileQuartz Veins from the Czech Erzgebirge. Geostand. Geoanal. Res. 41(1): 29-40

Durrant SF (1993) Alternatives to all-argon plasmas in inductively coupled plasma mass spectrometry (ICP-MS): an overview. Fresenius' Journal of Analytical Chemistry. 347(10): 389-392

Durrant SF (1994) Feasibility of improvement in analytical performance in laser ablation inductively coupled plasma-mass spectrometry (LA-ICP-MS) by addition of nitrogen to the argon plasma. Fresenius' Journal of Analytical Chemistry. 349(10): 768-771

Eggins SM, Kinsley LPJ, Shelley JMG (1998) Deposition and element fractionation processes during atmospheric pressure laser sampling for analysis by ICP-MS. Appl. Surf. Sci. 127-129: 278-286 
Flem B, Larsen RB, Grimstvedt A, Mansfeld J (2002) In situ analysis of trace elements in quartz by using laser ablation inductively coupled plasma mass spectrometry. Chem. Geol. 182(2-4): $237-247$

Fu J, Hu Z, Zhang W, Yang L, Liu Y, Li M, Zong K, Gao S, Hu S (2016) In situ sulfur isotopes ( $\delta 34 \mathrm{~S}$ and $\delta 33 \mathrm{~S}$ ) analyses in sulfides and elemental sulfur using high sensitivity cones combined with the addition of nitrogen by laser ablation MC-ICP-MS. Anal. Chim. Acta. 911: 14-26

Günther D, Heinrich CA (1999) Comparison of the ablation behaviour of $266 \mathrm{~nm} \mathrm{Nd:} \mathrm{YAG}$ and $193 \mathrm{~nm}$ ArF excimer lasers for LA-ICP-MS analysis. J. Anal. At. Spectrom. 14(9): $1369-1374$

Gagnon JE, Fryer BJ, Samson IM, Williams-Jones AE (2008) Quantitative analysis of silicate certified reference materials by LA-ICPMS with and without an internal standard. J. Anal. At. Spectrom. 23(11): 1529-1537

Gao S, Liu XM, Yuan HL, Hattendorf B, Günther D, Chen L, Hu SH (2002) Determination of Forty Two Major and Trace Elements in USGS and NIST SRM Glasses by Laser Ablation-Inductively Coupled Plasma-Mass Spectrometry. Geostandards Newsletter. 26(2): $181-196$

Garbe-Schönberg D, Müller S (2014) Nano-particulate pressed powder tablets for LA-ICP-MS. J. Anal. At. Spectrom. 29 (6): 990-1000

Guillong M, Heinrich CA (2007) Sensitivity enhancement in laser ablation ICP-MS using small amounts of hydrogen in the carrier gas. J. Anal. At. Spectrom. 22(12): 1488-1494

Guillong M, Sliwinski JT, Schmitt A, Forni F, Bachmann O (2016) U-Th Zircon Dating by 
Chapter 3.2 Signal enhancement in LA-ICP-MS analysis by guard electrode and the addition of nitrogen and hydrogen into carrier gas: A perspective from experiment

Laser Ablation Single Collector Inductively Coupled Plasma-Mass Spectrometry (LA-ICPMS). Geostand. Geoanal. Res. 40(3): 377-387

Holliday AE, Beauchemin D (2004) Spatial profiling of analyte signal intensities in inductively coupled plasma mass spectrometry. Spectrochimica Acta Part B: Atomic Spectroscopy. 59(3): 291-311

Hu Z, Liu Y, Li M, Gao S, Zhao L (2009) Results for Rarely Determined Elements in MPIDING, USGS and NIST SRM Glasses Using Laser Ablation ICP-MS. Geostand. Geoanal. Res. 33(3): 319-335

Hu ZC, Gao S, Liu YS, Hu SH, Chen HH, Yuan HL (2008) Signal enhancement in laser ablation ICP-MS by addition of nitrogen in the central channel gas. J. Anal. At. Spectrom. 23(8): 1093-1101

J. G. Mank A, R. D. Mason P (1999) A critical assessment of laser ablation ICP-MS as an analytical tool for depth analysis in silica-based glass samples. J. Anal. At. Spectrom. 14(8): $1143-1153$

Jackson SE, Pearson NJ, Griffin WL, Belousova EA (2004) The application of laser ablation-inductively coupled plasma-mass spectrometry to in situ U-Pb zircon geochronology. Chem. Geol. 211(1): 47-69

Jochum KP, Dingwell DB, Rocholl A, Stoll B, Hofmann AW, Becker S, Besmehn A, Bessette D, Dietze HJ, Dulski P (2000) The Preparation and Preliminary Characterisation of Eight Geological MPI-DING Reference Glasses for In-Situ Microanalysis. Geostandards Newsletter. 24(1): 87-133

Jochum KP, Nohl U, Herwig K, Lammel E, Stoll B, Hofmann AW (2005) GeoReM: a new 
Chapter 3.2 Signal enhancement in LA-ICP-MS analysis by guard electrode and the addition of nitrogen and hydrogen into carrier gas: A perspective from experiment

geochemical database for reference materials and isotopic standards. Geostand. Geoanal. Res. 29(3): $333-338$

Jochum KP, Stoll B, Herwig K, Willbold M, Hofmann AW, Amini M, Aarburg S, Abouchami W, Hellebrand E, Mocek B, Raczek I, Stracke A, Alard O, Bouman C, Becker S, Dücking M, Brätz H, Klemd R, de Bruin D, Canil D, Cornell D, de Hoog C-J, Dalpé C, Danyushevsky L, Eisenhauer A, Gao Y, Snow JE, Groschopf N, Günther D, Latkoczy C, Guillong M, Hauri EH, Höfer HE, Lahaye Y, Horz K, Jacob DE, Kasemann SA, Kent AJR, Ludwig T, Zack T, Mason PRD, Meixner A, Rosner M, Misawa K, Nash BP, Pfänder J, Premo WR, Sun WD, Tiepolo M, Vannucci R, Vennemann T, Wayne D, Woodhead JD (2006) MPI-DING reference glasses for in situ microanalysis: New reference values for element concentrations and isotope ratios. Geochemistry, Geophysics, Geosystems. 7(2): 1-44

Jochum KP, Weis U, Stoll B, Kuzmin D, Yang QC, Raczek I, Jacob DE, Stracke A, Birbaum K, Frick DA, Günther D, Enzweiler J (2011) Determination of Reference Values for NIST SRM 610-617 Glasses Following ISO Guidelines. Geostand. Geoanal. Res. 35(4): 397-429

Kaiyun C, Honglin Y, Zhian B, Chunlei Z, Mengning D (2014) Precise and Accurate In Situ Determination of Lead Isotope Ratios in NIST, USGS, MPI-DING and CGSG Glass Reference Materials using Femtosecond Laser Ablation MC-ICP-MS. Geostand. Geoanal. Res. 38(1): 5-21

Kimura J-I, Chang Q, Kawabata H (2013) Standardless determination of Nd isotope ratios in glasses and minerals using laser-ablation multiple-collector inductively coupled plasma mass spectrometry with a low-oxide molecular yield interface setup. J. Anal. At. Spectrom. 28(9): $1522-1529$

Koch J, Günther D (2011) Review of the State-of-the-Art of Laser Ablation Inductively Coupled Plasma Mass Spectrometry. Appl. Spectrosc. 65(5): 155A-162A 
Kosler J, Jackson S, Yang Z, Wirth R (2014) Effect of oxygen in sample carrier gas on laser-induced elemental fractionation in $\mathrm{U}-\mathrm{Th}-\mathrm{Pb}$ zircon dating by laser ablation ICP-MS. J. Anal. At. Spectrom. 29: 832-840

Latkoczy C, Günther D (2002) Enhanced sensitivity in inductively coupled plasma sector field mass spectrometry for direct solid analysis using laser ablation (LA-ICP-SFMS). J. Anal. At. Spectrom. 17(10): 1264-1270

Liu S, Hu Z, Gunther D, Ye Y, Liu Y, Gao S, Hu S (2014) Signal enhancement in laser ablation inductively coupled plasma-mass spectrometry using water and/or ethanol vapor in combination with a shielded torch. J. Anal. At. Spectrom. 29(3): 536-544

Liu YS, Hu ZC, Gao S, Gunther D, Xu J, Gao CG, Chen HH (2008) In situ analysis of major and trace elements of anhydrous minerals by LA-ICP-MS without applying an internal standard. Chem. Geol. 257(1-2): 34-43

Liu YS, Hu ZC, Li M, Gao S (2013) Applications of LA-ICP-MS in the elemental analyses of geological samples. Chinese Science Bulletin. 58(32): 3863-3878

Müller W, Shelley M, Miller P, Broude S (2009) Initial performance metrics of a new custom-designed ArF excimer LA-ICPMS system coupled to a two-volume laser-ablation cell. J. Anal. At. Spectrom. 24(2): 209-214

Macholdt DS, Jochum KP, Pöhlker C, Stoll B, Weis U, Weber B, Müller M, Kappl M, Buhre S, Kilcoyne ALD, Weigand M, Scholz D, Al-Amri AM, Andreae MO (2015) Microanalytical methods for in-situ high-resolution analysis of rock varnish at the micrometer to nanometer scale. Chem. Geol. 411: 57-68

Nonose NS, Matsuda N, Fudagawa N, Kubota M (1994) Some characteristics of polyatomic 
Chapter 3.2 Signal enhancement in LA-ICP-MS analysis by guard electrode and the addition of nitrogen and hydrogen into carrier gas: A perspective from experiment

ion spectra in inductively coupled plasma mass spectrometry. Spectrochimica Acta Part B: Atomic Spectroscopy. 49(10): 955-974

Pearce NJG, Perkins WT, Westgate JA, Gorton MP, Jackson SE, Neal CR, Chenery SP (1997) A Compilation of New and Published Major and Trace Element Data for NIST SRM 610 and NIST SRM 612 Glass Reference Materials. Geostandards Newsletter. 21(1): 115-144

Petrelli M, Laeger K, Perugini D (2016) High spatial resolution trace element determination of geological samples by laser ablation quadrupole plasma mass spectrometry: implications for glass analysis in volcanic products. Geosciences Journal. 20(6): 851-863

Raimondo T, Payne J, Wade B, Lanari P, Clark C, Hand M (2017) Trace element mapping by LA-ICP-MS: assessing geochemical mobility in garnet. Contributions to Mineralogy and Petrology. 172(4): DOI 10.1007/s00410-00017-01339-z

Russo RE, Mao X, Gonzalez JJ, Zorba V, Yoo J (2013) Laser Ablation in Analytical Chemistry. Analytical Chemistry. 85(13): 6162-6177

Sakata Ki, Kawabata K (1994) Reduction of fundamental polyatomic ions in inductively coupled plasma mass spectrometry. Spectrochimica Acta Part B: Atomic Spectroscopy. 49(10): 1027-1038

Segura M, Madrid Y, Camara C (2003) Elimination of calcium and argon interferences in iron determination by ICP-MS using desferrioxamine chelating agent immobilized in sol-gel and cold plasma conditions. J. Anal. At. Spectrom. 18(9): 1103-1108

Spandler C, Hammerli J, Sha P, Hilbert-Wolf H, Hu Y, Roberts E, Schmitz M (2016) MKED1: A new titanite standard for in situ analysis of $\mathrm{Sm}-\mathrm{Nd}$ isotopes and $\mathrm{U}-\mathrm{Pb}$ geochronology. Chem. Geol. 425: 110-126 
Stoll B, Jochum KP, Herwig K, Amini M, Flanz M, Kreuzburg B, Kuzmin D, Willbold M, Enzweiler J (2008) An automated iridium-strip heater for LA-ICP-MS bulk analysis of geological samples. Geostand. Geoanal. Res. 32(1): 5-26

Tomlinson EL, Thordarson T, Müller W, Thirlwall M, Menzies MA (2010) Microanalysis of tephra by LA-ICP-MS - Strategies, advantages and limitations assessed using the Thorsmörk ignimbrite (Southern Iceland). Chem. Geol. 279(3): 73-89

Tong X, Liu Y, Hu Z, Chen H, Zhou L, Hu Q, Xu R, Deng L, Chen C, Yang L, Gao S (2015) Accurate Determination of Sr Isotopic Compositions in Clinopyroxene and Silicate Glasses by LA-MC-ICP-MS. Geostand. Geoanal. Res. 40(1): 85-99

Ubide T, McKenna CA, Chew DM, Kamber BS (2015) High-resolution LA-ICP-MS trace element mapping of igneous minerals: In search of magma histories. Chem. Geol. 409: $157-168$

Wu S, Wang Y, Xu C, Yuan J (2016) Elemental Fractionation Studies of 193 nm ArF Excimer Laser Ablation System at High Spatial Resolution Mode. Chinese Journal of Analytical Chemistry. 44(7): 1035-1041

Xu L, Hu Z, Zhang W, Yang L, Liu Y, Gao S, Luo T, Hu SH (2015) In situ Nd isotope analyses in geological materials with signal enhancement and non-linear mass dependent fractionation reduction using laser ablation MC-ICP-MS. J. Anal. At. Spectrom. 30(1): $232-244$

Yuan HL, Gao S, Liu XM, Li HM, Gunther D, Wu FY (2004) Accurate U-Pb age and trace element determinations of zircon by laser ablation-inductively coupled plasma-mass 
Chapter 3.2 Signal enhancement in LA-ICP-MS analysis by guard electrode and the addition of nitrogen and hydrogen into carrier gas: A perspective from experiment

spectrometry. Geostand. Geoanal. Res. 28(3): 353-370

Zack T, Hogmalm KJ (2016) Laser ablation $\mathrm{Rb} / \mathrm{Sr}$ dating by online chemical separation of $\mathrm{Rb}$ and $\mathrm{Sr}$ in an oxygen-filled reaction cell. Chem. Geol. 437: 120-133

Zack T, Stockli DF, Luvizotto GL, Barth MG, Belousova E, Wolfe MR, Hinton RW (2011) In situ $\mathrm{U}-\mathrm{Pb}$ rutile dating by $\mathrm{LA}-\mathrm{ICP}-\mathrm{MS}: 208 \mathrm{~Pb}$ correction and prospects for geological applications. Contributions to Mineralogy and Petrology. 162(3): 515-530 
Chapter 4 Pushing the LA-ICP-MS analytical capability to sub$\mu \mathrm{m}$ scale spatial resolution: Elemental fractionation studies and ablation behavior investigations

\section{Chapter 4.1 Elemental Fractionation Studies of 193 nm ArF Excimer}

\section{Laser Ablation System at High Spatial Resolution}

Shitou $\mathrm{Wu}^{12,3}$, Yaping Wang ${ }^{1 *}$, Chunxue $\mathrm{Xu}^{1}$, Jihai Yuan ${ }^{1}$

${ }^{1}$ (National Research Center for Geoanalysis, Beijing 100037)

${ }^{2}$ (School of Earth Science, China University of Geosciences (Wuhan), Hubei Wuhan 430074)

${ }^{3}$ (Geoscience Center Göttingen, University of Göttingen, Göttingen, 37077, Germany)

\section{Graphical abstract}

The limit of detection, mass load effect, downhole induced fractionation and matrix effect of $193 \mathrm{~nm}$ ArF excimer laser at high spatial resolution $(<15 \mu \mathrm{m})$ were symmetrically investigated. The results showed that the downhole induced fractionation was negligible when the ratio of ablation depth to spot size was smaller than 1:1.

\section{Downhole induced fractionation}
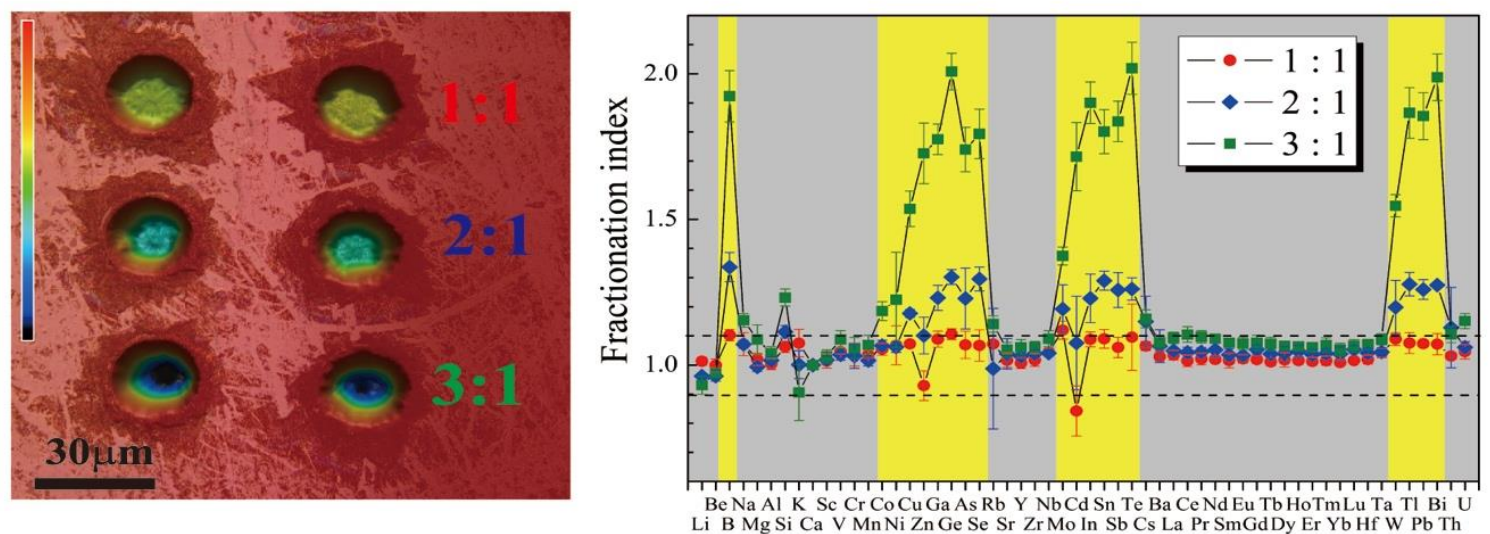


\begin{abstract}
Limits of detection (LODs), mass load effect, downhole induced fractionation and matrix effect of $193 \mathrm{~nm} \mathrm{ArF} \mathrm{excimer} \mathrm{laser} \mathrm{ablation} \mathrm{system} \mathrm{at} \mathrm{high} \mathrm{spatial} \mathrm{resolution} \mathrm{were}$ systematically investigated. Trace elements in GSD-1G, StHs6/80-G, and NIST612 were analyzed at $10 \mu \mathrm{m}$ spot size. The results showed that LODs decreased with increasing ablation diameter. LODs of some trace elements were in a range of $1 \sim 10 \mu \mathrm{g} / \mathrm{g}$ at $7 \mu \mathrm{m}$ spot size. Mass load effect was negatively correlated with corresponding oxide melting temperature, while positively correlated with elemental $1^{\text {st }}$ ionization potential. Downhole fractionation was negligible when the ratio of ablation depth versus spot size was smaller than 1:1. Matrix effect based on elemental pair method showed that there were no significant changes between spot sizes of $50 \mu \mathrm{m}$ and $10 \mu \mathrm{m}$ among investigated reference materials (NIST610, GSD-1G, ATHO-G, and StHs6/80-G). Based on NIAT610 as external standard and Ca as an internal standard, the analytical results of 36 trace elements in GSD-1G, StHs6/80-G and NIST612 at $10 \mu \mathrm{m}$ spot size matched well with the reference value. Generally, $10 \mu \mathrm{m}$ spatial resolution could satisfy the requirements of trace element analysis.
\end{abstract}

Keywords: LA-ICP-MS; Micro-analysis; Elemental fractionation; Matrix effects; Spatial resolution analysis. 


\subsubsection{Introduction}

Recently with increasing demands of elemental analysis at sub-micron scale such as mineral elemental mapping (Ubide et al., 2015), zonation mineral analysis (Gerdes and Zeh, 2009), diffusion studies (Selby and Creaser, 2004), measuring of ultra-small environmental tracers (tephra (Tomlinson et al., 2010), rock varnish (Macholdt et al., 2015) and fish otoliths (Sanborn and Telmer, 2003) et al.), high spatial resolution analysis is drawing more and more attention. Laser ablation-inductively coupled plasma mass spectrometry (LA-ICP-M) as a prominent microanalysis technique has been widely used for measurement of elements and isotopic ratios in analytical geochemistry (Garbe-Schönberg and Müller, 2014; Koch and Günther, 2011; Liu et al., 2013). Advantages of this approach include high spatial resolution, low limit of detection (LOD) and rapid throughput. Elemental fractionation is one of the main bottlenecks restricting applications of this technique (Zhang et al., 2016). $193 \mathrm{~nm}$ ArF excimer laser offers qualities like low cost and good stability (Müller et al., 2009), thus it occupies a large proportion in the LA-ICP-MS laboratory. Elemental fractionation study of $193 \mathrm{~nm}$ ArF excimer laser at high spatial resolution is necessary and of significance.

Challenges faced by LA-ICP-MS at high spatial resolution $(<15 \mu \mathrm{m})$ include low sensitivity, larger uncertainty, difficulty in focusing and serious elemental fractionation. Fan et al (Fan et al., 2015) reported that the ablation pits were exhibited as ellipse shape at $7 \mu \mathrm{m}$ spot size, which may due to laser defocus during the ablation process. Elemental fractionation mainly stems from three aspects (Zhang et al., 2016), (a) thermal and downhole effects during ablation (Guillong et al., 2003; Machida et al., 2015; Yuan et al., 2012), (b) particles transmission and losses during transportation (Garcia et al., 2007; Koch et al., 2008), (c) mass load effects during the ionization process in ICP (Fietzke and Frische, 2016). The smaller ablation diameter and less amount of ablation materials at high spatial resolution lead to a more serious downhole fractionation and mass load effect. Fryer et al. (1995) reported the fractionation index (FI) to quantify the elemental fraction. That is defined as the ratio of the total counts determined at the second half of measurement to the first half and normalized to 
internal element Ca. Kroslakova and Günther (2007) showed that an increase of the mass load of the ICP by a factor of 16 led to a decrease in intensity ratios (e.g., $\mathrm{Cu} / \mathrm{Ca}$ ) by a percentage up to 25. Current studies related with elemental fractionation at high spatial resolution are rare (Fricker et al., 2011; Hu et al., 2011; Li et al., 2015).

By using international reference materials and based on previous literature ( $\mathrm{Li}$ et al., 2015; Tomlinson et al., 2010), the LOD, mass load effect, downhole induced fractionation and matrix effects of $193 \mathrm{~nm}$ ArF excimer laser ablation system at high spatial resolution was investigated in this study. Trace elements in GSD-1G, StHs6/80-G, and NIST612 were measured at $10 \mu \mathrm{m}$ spots size.

\subsubsection{Experimental}

\subsubsection{Instrumentation}

All experiments were conducted on an ELEMENT 2 sector field ICP-MS (Thermo Scientific, USA) in combination with a $193 \mathrm{~nm}$ ArF excimer laser ablation system (RESOlution M-50, Australian Scientific Instruments, Australia) at LA-ICP-MS Laboratory, University of Göttingen. Details of instrument operating conditions are summarized in Table 1. The torch position and carrier gas flow was optimized by using NIST612 as the calibration materials to obtain the maximum signals of ${ }^{7} \mathrm{Li} 、{ }^{139} \mathrm{La}$ and ${ }^{232} \mathrm{Th}$ while keeping $\mathrm{U} / \mathrm{Th}$ ratio close to 1 and $\mathrm{ThO} / \mathrm{Th}$ and $\mathrm{Ca}^{2+} / \mathrm{Ca}^{+}$lower than $0.5 \% .77$ isotopes were scanned in a mass range from ${ }^{7} \mathrm{Li}$ to ${ }^{238} \mathrm{U}$ in one second.

Table1 Operation conditions of LA-ICP-MS system

\begin{tabular}{cccc}
\hline & Laser ablation system & \multicolumn{2}{c}{ ICP-MS } \\
\hline Laser type & Excimer & ICP-MS & Element 2 \\
wavelength & $193 \mathrm{~nm}$ & RF Power & $1500 \mathrm{~W}$ \\
Pulse time & $20 \mathrm{~ns}$ & Guard electrode & Grounded \\
Energy density & $\sim 3.0 \mathrm{~J} / \mathrm{cm}^{2}$ & Coolant gas flow & $15.00 \mathrm{~L} / \mathrm{min}$ \\
Frequency & $5 \mathrm{~Hz}, 10 \mathrm{~Hz}$ & Auxiliary gas flow & $1.00 \mathrm{~L} / \mathrm{min}$ \\
Ablation cell & Laurin Technic S-155 & Sampling gas flow & $0.85 \mathrm{~L} / \mathrm{min}$ \\
Spot size & $7 \mu \mathrm{m}, 10 \mu \mathrm{m}, 15 \mu \mathrm{m}, 33 \mu \mathrm{m}, 50 \mu \mathrm{m}, 75 \mu \mathrm{m}$ & Segment duration & $10 \mathrm{~ms}$ \\
Ablation gas flow & $0.45 \mathrm{~L} / \mathrm{min}$ & Detector & Counting and analog \\
Ablation time & $35 \mathrm{~s}, 180 \mathrm{~s}$ & Resolution $(\mathrm{M} / \Delta \mathrm{M})$ & Low $(\sim 300)$ \\
\hline
\end{tabular}




\subsubsection{Samples}

Samples used in this study are international reference materials. That includes NIST 610, NIST 612, GSD-1G, StHs6/80-G and ATHO-G. Reference values and uncertainties are cited from literatures (Jochum et al., 2006; Jochum et al., 2011) and GeoReM database (http://georem.mpch- mainz.gwdg.de/). Samples were polished to $1 \mu \mathrm{m}$, cleaned with ethanol in the ultrasonic machine and dried with $\mathrm{N}_{2}$ before measurement.

\subsubsection{Data acquisition and evaluation}

Two ablation modes including discrete spot and line scanning were used for data collection. Ablation times are $180 \mathrm{~s}$ and $35 \mathrm{~s}$ for downhole fractionation and matrix effect studies at discrete mode. Ablation frequency was set as $5 \mathrm{~Hz}$. Backgrounds were recorded in 20 s before and after ablation. Line scanning speed and ablation frequency were $15 \mu \mathrm{m} / \mathrm{s}$ and $10 \mathrm{~Hz}$ respectively at line scanning mode. Ablation time is $35 \mathrm{~s}$.

Data reduction was conducted in Iolite 3.0 and Microsoft Excel. Iolite3.0 was used for instrument drift correction and data normalization. Elemental fractionation data processing was based on Excel.

\subsubsection{Results and discussion}

\subsubsection{Limits of detection}

The limit of detection (LODs) is closely related to ablation parameters such as spot size, energy density, and frequency. In general, more ablated materials will lead to better LODs. Thus LODs at high spatial resolution $(<15 \mu \mathrm{m})$ is higher than normal mode $(>50 \mu \mathrm{m})$. Fig. 1 shows the LODs of 46 elements at different spot size including $7 \mu \mathrm{m}, 10 \mu \mathrm{m}, 15 \mu \mathrm{m}, 33 \mu \mathrm{m}$, $50 \mu \mathrm{m}$ and $75 \mu \mathrm{m}$. The LODs were calculated based on NIST612. LODs increase with decreasing ablation diameters (Fig.1). Most elements LODs are lower than $0.01 \mu \mathrm{g} / \mathrm{g}$ at $50 \mu \mathrm{m}$ spot size, in a range of $0.1 \sim 1 \mu \mathrm{g} / \mathrm{g}$ at $10 \mu \mathrm{m}$ spot size and in a range of $1 \sim 10 \mu \mathrm{g} / \mathrm{g}$ at $7 \mu \mathrm{m}$ spot size. Too high LODs at $7 \mu \mathrm{m}$ spot size make this technique not well suited for low-level elemental analysis. Various authors (Chen et al., 2015; Guillong and Heinrich, 2007; Hu et al., 
Chapter 4.1 Elemental Fractionation Studies of 193 nm ArF Excimer Laser Ablation System at High Spatial Resolution

2008) reported that by introducing $\mathrm{H}_{2}, \mathrm{~N}_{2}$ into ICP and using guard electrode to increase sensitivity, simultaneous analysis of trace elements at high spatial resolution with LA-ICP-MS could be conducted.

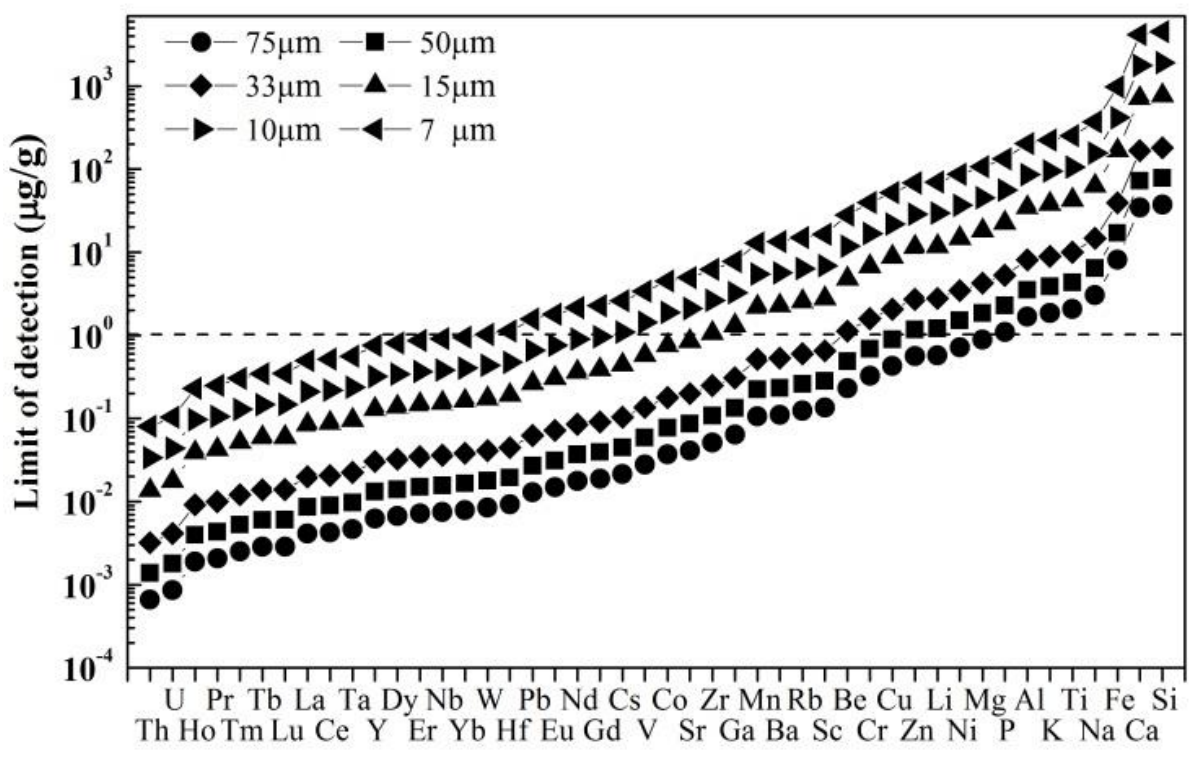

Fig.1 The limit of detection of LA-ICP-MS system at different spot sizes

\subsubsection{Mass load effect}

Mass load effect has been defined as an increase in ICP mass load led a change in intensity ratio. $\mathrm{Li}$ et al. (2015) reported a gradual decrease in $\mathrm{Cu} / \mathrm{Ca}, \mathrm{Zn} / \mathrm{Ca}$ and $\mathrm{Pb} / \mathrm{Ca}$ up to $20 \%$ with an increase in ICP mass load by using a $193 \mathrm{~nm}$ ArF excimer laser ablation system, however, the author did not study spot size lower than $16 \mu \mathrm{m}$. To eliminate laser induced elemental fractionation effects, all data were collected at line scanning mode. Different spots sizes $(7 \mu \mathrm{m}$, $10 \mu \mathrm{m}, 15 \mu \mathrm{m}, 33 \mu \mathrm{m}, 50 \mu \mathrm{m}$, and $75 \mu \mathrm{m})$ represent distinct mass loads introduced into plasma.

Elemental signals were first normalized to ${ }^{43} \mathrm{Ca}$. Mass load induced fractionation index (FI) is defined as the ratio of element/Ca in certain spot size to $75 \mu \mathrm{m}$. Selecting element/Ca in $75 \mu \mathrm{m}$ as denominator could eliminate signal uncertainty due to a small spot size (like $7 \mu \mathrm{m}$ ). Fig.2 shows FI of selected elements as a function of ICP mass load. FI of refractory lithophile elements is nearly independent of mass load, while for volatile elements like $\mathrm{Cu}, \mathrm{Ga}, \mathrm{Mo}, \mathrm{Cs}$ and $\mathrm{Pb}, \mathrm{FI}$ increased with mass load decreasing. With a decrease in ICP mass load down to 
19.36\% (33 $\mu \mathrm{m}$ spot size), the FI of volatile elements did not essentially change and gradually increased in a range of $4.00 \%-0.87 \%(15 \sim 7 \mu \mathrm{m})$. When down to $0.87 \%(7 \mu \mathrm{m})$, the FI increased to a range of 1.15 1.35. The results illustrated that ionization process of ablation materials in ICP was not completely conducted (Kroslakova and Günther, 2007). Volatile elements were ionized more completely than $\mathrm{Ca}$ with decreasing ICP mass load.

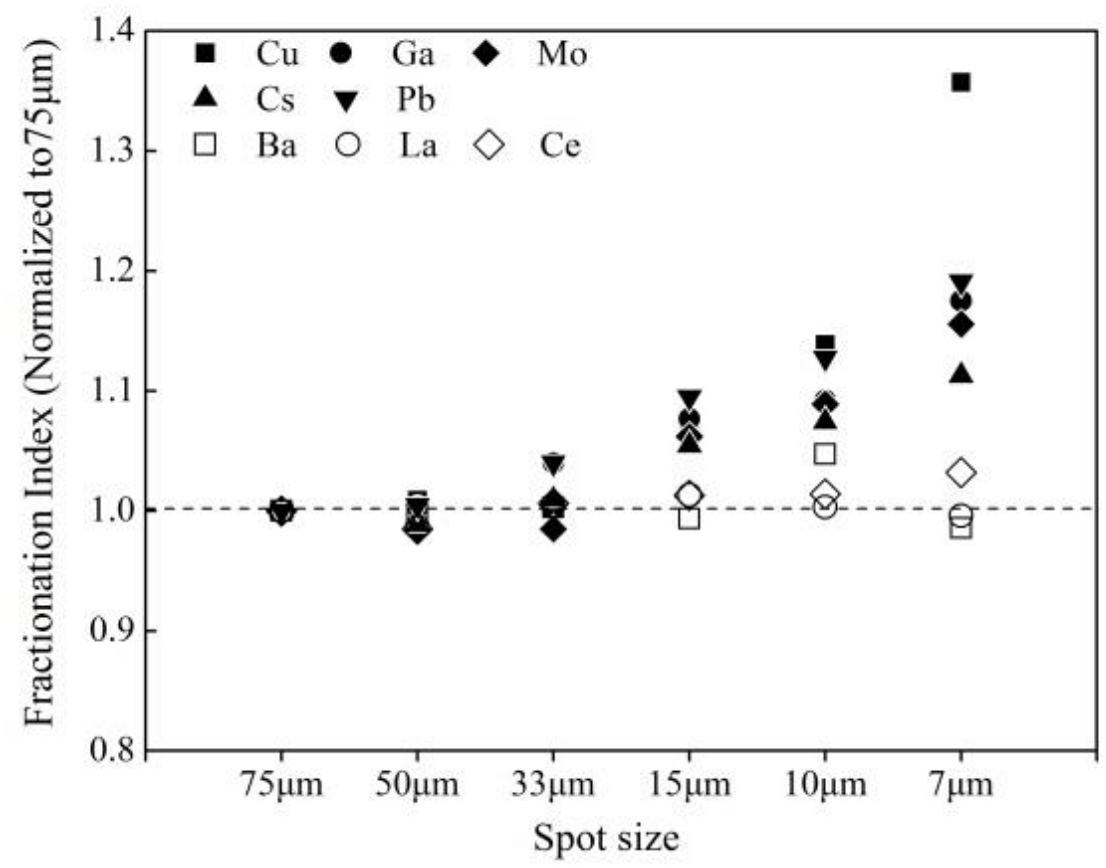

Fig.2 Fractionation index of selected elements as a function of ICP mass load

Further studies show that mass load effects are controlled by oxide melting point and $1^{\text {st }}$ ionization potential (Ho et al., 2015). Fig.2 illustrates FI was negatively correlated with corresponding oxide melting temperature, which indicated that elements with lower oxide melting temperature may be more easily "atomized". FI was positively correlated with elemental $1^{\text {st }}$ ionization potential that indicated that the ionization efficiency improved, especially for those elements with higher $1^{\text {st }}$ ionization potential. 

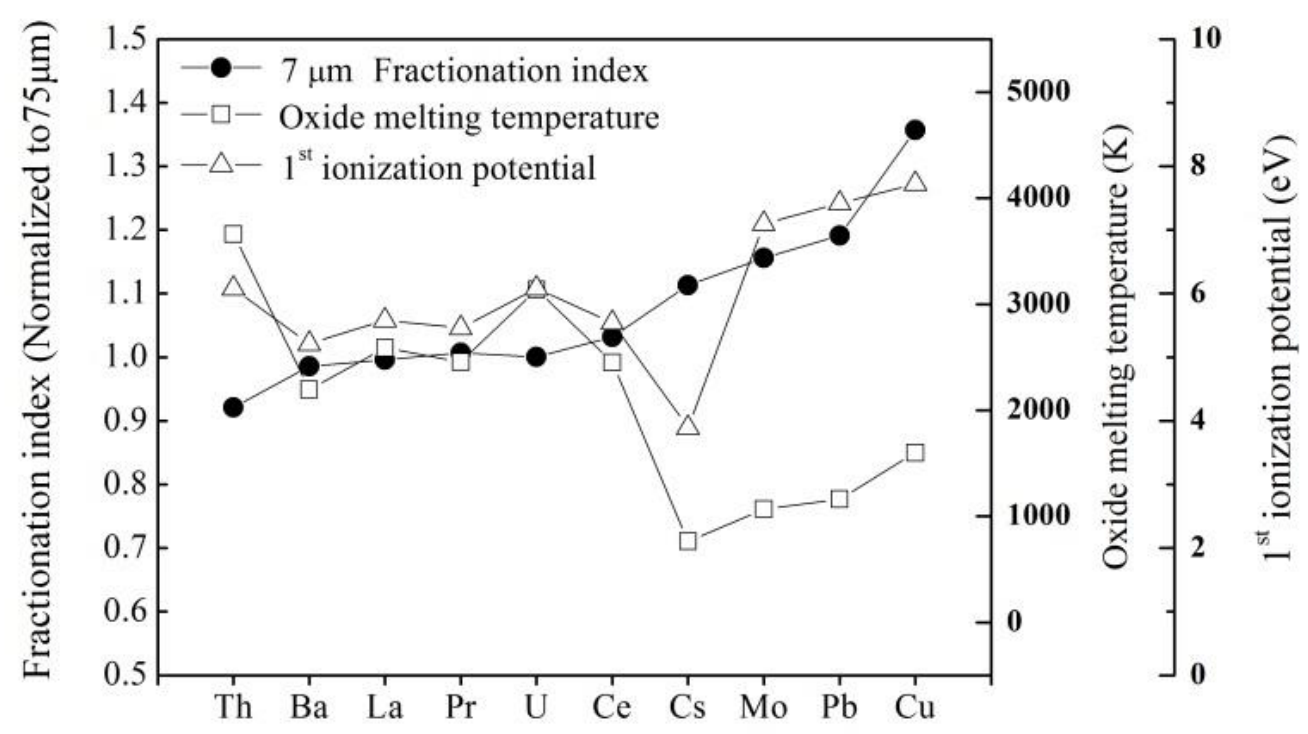

Fig.3 The fractionation index ( $7 \mu \mathrm{m}$ spot size) as function of oxide melting temperature and $1^{\text {st }}$ ionization potential

\subsubsection{Downhole induced fractionation}

Downhole induced fractionation has always been a key research topic for this technique (Borisov et al., 2000; Eggins et al., 1998; Mank and Mason, 1999). That directly affects the accuracy of analysis results (Kuhn and Günther, 2003; Longerich et al., 1996; Luo et al., 2015). More serious fractionation was found at high spatial resolution mode due to the small ablation diameter. Ensuring sufficient signal intensity as well as the smallest possible ablation diameter, $23 \mu \mathrm{m}$ spot diameter was selected to investigate the downhole fractionation.

The depth and diameter of ablation pits were measured by an optical microscope (Leica DMRX). The results showed that the ablation rate is $0.11 \mu \mathrm{m} /$ pulse under a $3.0 \mathrm{~J} / \mathrm{cm}^{2}$ laser energy density. The depth of ablation pits is $99 \mu \mathrm{m}$ after a continuous 180 s ablation without considering defocus effects during the ablation processing. Fig. 4 shows the ratio of elemental intensity to ${ }^{43} \mathrm{Ca}$ as a function of ablation depth/spot size. $\mathrm{Cu} / \mathrm{Ca}$ and $\mathrm{Zn} / \mathrm{Ca}$ increased with increasing ablation depth / spot size, while the $\mathrm{Sc} / \mathrm{Ca}$ and $\mathrm{Ce} / \mathrm{Ca}$ remain constant. Downhole induced fractionation index was defined as Fryer et al. (1995) reported. Fig.5 shows the FI of 
46 elements at distinct ablation depth/spot size (1:1,2:1 and 3:1). The results illustrated FI of most elements are in a range of 0.9-1.1 under ablated depth/spot size (1:1). With the increase of ablation depth / spot size to 3:1, FI of volatile elements (B), chalcophile elements $(\mathrm{Cu}, \mathrm{Zn}$, and $\mathrm{Ga}$ et al.), and siderophile elements (Co, $\mathrm{Ni}$ and $\mathrm{W}$ et al.) increased significantly, however, FI of the lithophile elements (Sc, Sr and REEs et al.) still remained constant. These results are consistent with previous studies (Fryer et al., 1995; Hu et al., 2011; Mank and Mason, 1999). Fractionation effects are negligible when the ablation depth/spot size is smaller than $1: 1$, although the downhole fractionation turns to be serious with the increase of ablation time,

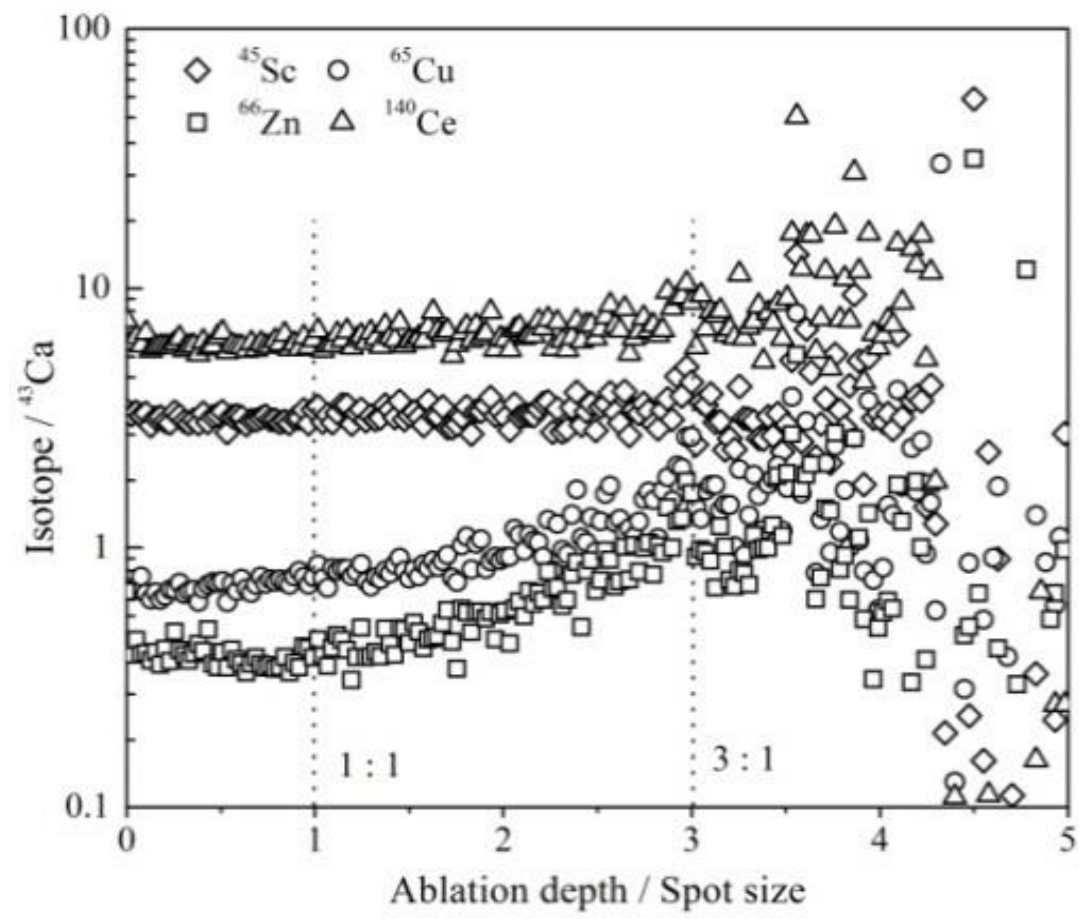

Fig.4 The ratio of elements intensity to ${ }^{43} \mathrm{Ca}$ as a function of ablation depth/spot size

Table 2 shows a correlation between spot size and ablated time which represents ablation depth/spot size equal to 1:1 at our laser parameters in this study. The downhole fractionation effect could be ignorable when the ablation duration is smaller than $18 \mathrm{~s}$ under $10 \mu \mathrm{m}$ spot size. 


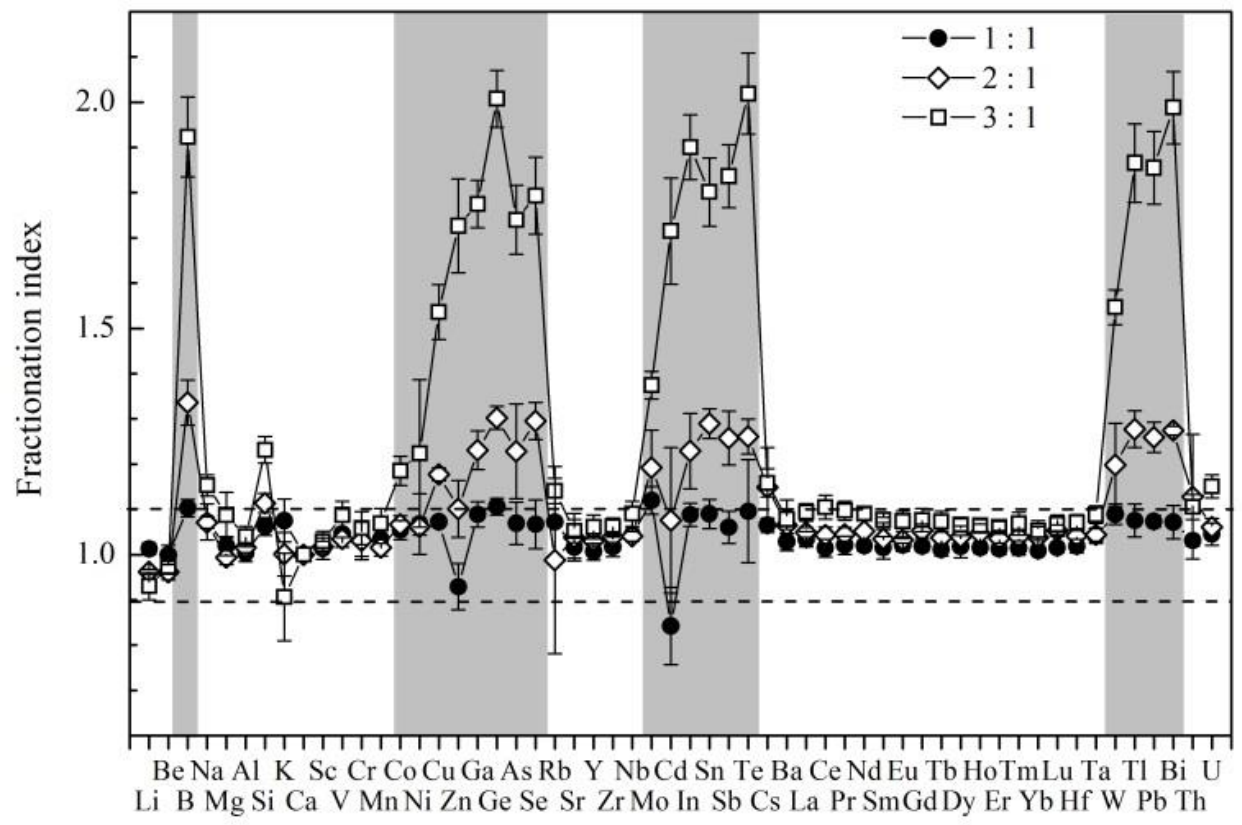

Fig.5 The fractionation index of different elements at ratio of ablated depth to spot size (1:1, $2: 1$ and $3: 1)$

Table 2 Correlation of spot size and ablated time

\begin{tabular}{cc}
\hline Spot size $(\mu \mathrm{m})$ & Ablation time $(\mathrm{s})$ \\
\hline 75 & 136 \\
50 & 91 \\
33 & 60 \\
23 & 42 \\
15 & 27 \\
10 & 18 \\
7 & 13 \\
\hline
\end{tabular}

\subsubsection{Matrix effect}

Matrix effect between reference materials and samples is one of the main factors influencing the accuracy of LA-ICP-MS analytical results, especially when the non-matrix matched reference materials are applied for calibration (Hu et al., 2011; Jochum et al., 2014; Yuan et al., 2011). Yuan et al. (2015) proposed a method to quantify the matrix effects between reference materials and samples. That is described by the degree of the linear correlation coefficient $\left(\mathrm{R}^{2}\right)$ and relative standard deviation (RSD) of relative sensitivity factors (RSF). RSF is the ratio of intensity to the concentration of determined elements normalized to the 
Chapter 4.1 Elemental Fractionation Studies of 193 nm ArF Excimer Laser Ablation System at High Spatial Resolution

internal standard element. Matrix effects of NIST 610, GSD-1G, ATHO-G and StHs6/80-G were investigated based on $\mathrm{Ca}$ as an internal standard at a spot size of $50 \mu \mathrm{m}$ and $10 \mu \mathrm{m}$. Nine elements from lower to high mass were selected for this study. Results are showed in Fig.3.

Table 3 Linear correlation coefficient $\left(\mathrm{R}^{2}\right)$ and the relative sensitivity factors (RSD) of relative sensitivity factor (RSF) of different elements at $50 \mu \mathrm{m}$ and $10 \mu \mathrm{m}$ spot sizes

\begin{tabular}{ccccc}
\hline Spot size & \multicolumn{2}{c}{$50 \mu \mathrm{m}$} & \multicolumn{2}{c}{$10 \mu \mathrm{m}$} \\
Elements & $\mathrm{R}^{2}$ & $\mathrm{RSD}(\%)$ & $\mathrm{R}^{2}$ & $\mathrm{RSD}(\%)$ \\
\hline $\mathrm{Al}$ & 0.9996 & 2.58 & 0.9999 & 4.54 \\
$\mathrm{Mn}$ & 1.0000 & 2.50 & 0.9997 & 4.22 \\
$\mathrm{Zn}$ & 0.9980 & 3.87 & 0.9936 & 8.58 \\
$\mathrm{Sr}$ & 0.9995 & 1.45 & 0.9991 & 1.52 \\
$\mathrm{Zr}$ & 0.9998 & 4.13 & 1.0000 & 3.23 \\
$\mathrm{Ba}$ & 1.0000 & 1.45 & 0.9998 & 4.34 \\
$\mathrm{Ce}$ & 0.9999 & 1.75 & 0.9995 & 2.09 \\
$\mathrm{~Pb}$ & 0.9999 & 4.19 & 0.9937 & 12.48 \\
$\mathrm{U}$ & 1.0000 & 1.87 & 0.9999 & 6.32 \\
\hline
\end{tabular}

RSD at $10 \mu \mathrm{m}$ is larger than at $50 \mu \mathrm{m}$, which is mostly due to the larger measurement uncertainty at $10 \mu \mathrm{m}$ and not caused by the matrix effects. Most $\mathrm{R}^{2}$ of linear correlation coefficients at pot size of $50 \mu \mathrm{m}$ and $10 \mu \mathrm{m}$ show no essential difference, which indicates that the matrix effects are almost same at a spot size of $50 \mu \mathrm{m}$ and $10 \mu \mathrm{m}$.

\subsubsection{Measurement of GSD-1G, StHs6/80-G, and NIST612 at $10 \mu \mathrm{m}$ spot size}

Trace elements of GSD-1G, StHs6/80-G, and NIST612 were measured at $10 \mu \mathrm{m}$ spot size. Laser parameters were energy density $\left(3.0 \mathrm{~J} / \mathrm{cm}^{2}\right)$, ablation frequency $(5 \mathrm{~Hz})$ and ablation duration (18s). The calibration was based on NST610 as reference materials and Ca as the internal standard. Results are shown in Fig.4. 
Table 3 Results of GSD-1G, StHs6/80-G, and NIST612 at spot size of $10 \mu \mathrm{m}$

\begin{tabular}{|c|c|c|c|c|c|c|}
\hline \multirow[b]{2}{*}{ Elements } & \multicolumn{2}{|c|}{ GSD-1G } & \multicolumn{2}{|c|}{ StHs6/80-G } & \multicolumn{2}{|c|}{ NIST612 } \\
\hline & $\begin{array}{c}\text { Certified value } \pm \\
\text { Uncertainty }(2 \sigma), \\
\mu \mathrm{g} / \mathrm{g}\end{array}$ & $\begin{array}{c}\text { Concentration } \pm 2 \mathrm{~s}, \\
\mu \mathrm{g} / \mathrm{g}\end{array}$ & $\begin{array}{c}\text { Certified value } \pm \\
\text { Uncertainty }(2 \sigma), \\
\mu \mathrm{g} / \mathrm{g}\end{array}$ & $\begin{array}{c}\text { Concentration } \pm 2 \mathrm{~s}, \\
\mu \mathrm{g} / \mathrm{g}\end{array}$ & $\begin{array}{c}\text { Certified value } \pm \\
\text { Uncertainty }(2 \sigma), \\
\mu \mathrm{g} / \mathrm{g}\end{array}$ & $\begin{array}{c}\text { Concentration } \pm 2 \mathrm{~s}, \\
\mu \mathrm{g} / \mathrm{g}\end{array}$ \\
\hline $\mathrm{Li}$ & $43.0 \pm 6.00$ & - & $20.7 \pm 2.30$ & - & $40.2 \pm 1.30$ & - \\
\hline $\mathrm{Be}$ & $46.0 \pm 5.00$ & - & $1.20 \pm 0.100$ & - & $37.5 \pm 1.50$ & - \\
\hline B & $50.0 \pm 20.0$ & - & $11.8 \pm 1.30$ & - & $34.3 \pm 1.70$ & - \\
\hline $\mathrm{Sc}$ & $52.0 \pm 2.00$ & $49.6 \pm 6.87$ & $11.5 \pm 0.800$ & - & $39.9 \pm 2.50$ & $41.3 \pm 4.64$ \\
\hline V & $44.0 \pm 2.00$ & $48.5 \pm 9.40$ & $90.3 \pm 6.70$ & $99.0 \pm 16.1$ & $38.8 \pm 1.20$ & $39.2 \pm 4.40$ \\
\hline $\mathrm{Cr}$ & $42.0 \pm 3.00$ & $68.5 \pm 14.3$ & $16.9 \pm 3.30$ & - & $36.4 \pm 1.50$ & - \\
\hline Co & $40.0 \pm 2.00$ & $51.4 \pm 9.98$ & $13.2 \pm 1.10$ & - & $35.5 \pm 1.00$ & $29.4 \pm 3.22$ \\
\hline $\mathrm{Ni}$ & $58.0 \pm 4.00$ & - & $13.2 \pm 1.10$ & - & $38.8 \pm 0.200$ & - \\
\hline $\mathrm{Cu}$ & $42.0 \pm 2.00$ & $57.3 \pm 18.5$ & $41.5 \pm 8.30$ & $55.3 \pm 15.6$ & $37.8 \pm 1.50$ & $35.7 \pm 14.6$ \\
\hline $\mathrm{Zn}$ & $54.0 \pm 2.00$ & - & $67.0 \pm 7.00$ & - & $39.1 \pm 1.70$ & - \\
\hline $\mathrm{Rb}$ & $37.3 \pm 0.400$ & $51.9 \pm 5.29$ & $30.7 \pm 1.70$ & $38.4 \pm 3.83$ & $31.4 \pm 0.400$ & $31.5 \pm 2.91$ \\
\hline $\mathrm{Sr}$ & $69.4 \pm 0.700$ & $73.7 \pm 12.3$ & $482 \pm 8.00$ & $504 \pm 78.7$ & $78.4 \pm 0.200$ & $77.2 \pm 4.98$ \\
\hline $\mathrm{Y}$ & $42.0 \pm 2.00$ & $40.1 \pm 4.61$ & $11.4 \pm 0.400$ & $10.6 \pm 3.39$ & $38.3 \pm 1.40$ & $40.8 \pm 5.61$ \\
\hline $\mathrm{Zr}$ & $42.0 \pm 2.00$ & - & $118 \pm 3.00$ & $110 \pm 26.1$ & $37.9 \pm 1.20$ & - \\
\hline $\mathrm{Nb}$ & $42.0 \pm 3.00$ & $48.1 \pm 5.57$ & $6.94 \pm 0.250$ & $7.53 \pm 2.09$ & $38.9 \pm 2.10$ & $38.0 \pm 2.97$ \\
\hline Cs & $32.0 \pm 2.00$ & $42.3 \pm 4.47$ & $1.75 \pm 0.110$ & - & $42.7 \pm 1.80$ & $41.9 \pm 2.12$ \\
\hline $\mathrm{Ba}$ & $67.0 \pm 1.00$ & $76.4 \pm 21.9$ & $298 \pm 9.00$ & $326 \pm 56.8$ & $39.3 \pm 0.900$ & $41.4 \pm 10.6$ \\
\hline $\mathrm{La}$ & $39.1 \pm 0.400$ & $39.3 \pm 4.99$ & $12.0 \pm 0.300$ & $11.8 \pm 1.68$ & $36.0 \pm 0.700$ & $36.6 \pm 3.91$ \\
\hline $\mathrm{Ce}$ & $41.4 \pm 0.400$ & $43.1 \pm 6.70$ & $26.1 \pm 0.700$ & $26.8 \pm 4.97$ & $38.4 \pm 0.700$ & $38.4 \pm 1.90$ \\
\hline $\operatorname{Pr}$ & $45.0 \pm 1.00$ & $47.2 \pm 5.77$ & $3.20 \pm 0.060$ & $3.26 \pm 1.39$ & $37.9 \pm 1.00$ & $38.6 \pm 4.51$ \\
\hline $\mathrm{Nd}$ & $44.7 \pm 0.500$ & $45.8 \pm 10.5$ & $13.0 \pm 0.300$ & $14.6 \pm 7.10$ & $35.5 \pm 0.700$ & $37.8 \pm 2.98$ \\
\hline $\mathrm{Sm}$ & $47.8 \pm 0.500$ & $48.3 \pm 8.45$ & $2.78 \pm 0.050$ & $2.82 \pm 1.49$ & $37.7 \pm 0.800$ & $36.5 \pm 9.55$ \\
\hline $\mathrm{Eu}$ & $41.0 \pm 2.00$ & $41.4 \pm 5.57$ & $0.953 \pm 0.022$ & - & $35.6 \pm 0.800$ & $35.6 \pm 5.10$ \\
\hline $\mathrm{Gd}$ & $50.7 \pm 0.500$ & $44.7 \pm 6.88$ & $2.59 \pm 0.090$ & - & $37.3 \pm 0.900$ & $40.01 \pm 4.12$ \\
\hline $\mathrm{Tb}$ & $47.0 \pm 2.00$ & $47.0 \pm 6.50$ & $0.371 \pm 0.011$ & - & $37.6 \pm 1.10$ & $37.9 \pm 3.60$ \\
\hline Dy & $51.2 \pm 0.500$ & $54.0 \pm 7.45$ & $2.22 \pm 0.060$ & - & $35.5 \pm 0.700$ & $35 \pm 6.13$ \\
\hline Ho & $49.0 \pm 2.00$ & $50.0 \pm 5.78$ & $0.420 \pm 0.011$ & $0.639 \pm 0.203$ & $38.3 \pm 0.800$ & $39.6 \pm 2.95$ \\
\hline $\mathrm{Er}$ & $40.1 \pm 0.400$ & $39.1 \pm 5.16$ & $1.18 \pm 0.040$ & - & $38.0 \pm 0.900$ & $43 \pm 5.39$ \\
\hline $\mathrm{Tm}$ & $49.0 \pm 2.00$ & $50.1 \pm 6.24$ & $0.172 \pm 0.007$ & - & $36.8 \pm 0.600$ & $38.4 \pm 3.31$ \\
\hline $\mathrm{Yb}$ & $50.9 \pm 0.500$ & $50.3 \pm 11.4$ & $1.13 \pm 0.030$ & - & $39.2 \pm 0.900$ & $41.4 \pm 7.73$ \\
\hline $\mathrm{Lu}$ & $51.5 \pm 0.500$ & $50.6 \pm 6.28$ & $0.168 \pm 0.006$ & - & $37.0 \pm 0.900$ & $37.5 \pm 3.58$ \\
\hline $\mathrm{Hf}$ & $39.0 \pm 2.00$ & $39.9 \pm 4.80$ & $3.07 \pm 0.090$ & $3.15 \pm 1.71$ & $36.7 \pm 1.20$ & $39.9 \pm 5.47$ \\
\hline $\mathrm{Ta}$ & $40.0 \pm 4.00$ & $44.5 \pm 3.56$ & $0.420 \pm 0.015$ & - & $37.6 \pm 1.90$ & $39.1 \pm 6.10$ \\
\hline $\mathrm{Pb}$ & $50.0 \pm 2.00$ & $65.7 \pm 7.53$ & $10.3 \pm 0.900$ & $12.9 \pm 3.42$ & $38.6 \pm 0.200$ & $39.3 \pm 4.90$ \\
\hline Th & $41.0 \pm 2.00$ & $43.2 \pm 5.65$ & $2.28 \pm 0.070$ & $2.26 \pm 0.972$ & $37.8 \pm 0.080$ & $39.1 \pm 3.24$ \\
\hline $\mathrm{U}$ & $41.0 \pm 2.00$ & $48.2 \pm 5.53$ & $1.01 \pm 0.040$ & $1.13 \pm 0.445$ & $37.4 \pm 0.080$ & $36.8 \pm 1.87$ \\
\hline
\end{tabular}

Note: "2s" represents two times of standard deviation $(n=8)$; "-" represents lower than the limit of detection. 
The calculated element concentrations are in general agreement with the reference value (Fig.4). However, a larger bias still exists for the elements concentrations of $\mathrm{Cr}, \mathrm{Cu}, \mathrm{Rb}$ and $\mathrm{Cs}$ in GSD-1G, Rb in StHs6/80-G and Co in NIST612. This phenomenon may be due to the larger analytical uncertainties at $10 \mu \mathrm{m}$ spot size. In general, the accurate measurement of trace elements could be conducted at $10 \mu \mathrm{m}$ spot size under our laser parameters.

\subsubsection{Conclusions}

In this study, the LODs, mass load effect, downhole induced fractionation and matrix effect of ArF excimer laser ablation system at high spatial resolution were systematically investigated. Results showed LODs is higher at smaller ablation diameters. LODs of most determined elements are better than $0.010 \mu \mathrm{g} / \mathrm{g}$ when spot size is larger than $50 \mu \mathrm{m}$, while at a range of $1 \sim 10 \mu \mathrm{g} / \mathrm{g}$ with spot size down to $7 \mu \mathrm{m}$. Mass load effect is closely related to the corresponding oxide melting temperature and $1^{\text {st }}$ ionization potential. Elements with low oxide melting temperature and higher $1^{\text {st }}$ ionization potential suffered more serious mass load effect. Downhole induced fractionation could be ignored when the ablation depth/spot size is smaller than 1:1. Matrix effect results indicated that the degree of matrix-related effects did not change from $50 \mu \mathrm{m}$ to $10 \mu \mathrm{m}$ spot size. Analytical results of trace elements in GSD-1G, StHs6/80-G, and NIST612 at $10 \mu \mathrm{m}$ are in general agreement with the reference values. A spatial resolution of $10 \mu \mathrm{m}$ is practical for accurate analysis of geological glasses. With the development of signal enhancement studies, the spatial resolution of LA-ICP-MS could be down to lower than $10 \mu \mathrm{m}$. 


\section{Reference}

Borisov OV, Mao X, Russo RE (2000) Effects of crater development on fractionation and signal intensity during laser ablation inductively coupled plasma mass spectrometry. Spectrochimica Acta Part B: Atomic Spectroscopy. 55(11): 1693-1704

Chen T, Hu Z, Liu S, Liu Y, Gao S, Li M, Zong K, Chen H, Hu S (2015) Improved performance of a shielded torch using ethanol in inductively coupled plasma-sector field mass spectrometry. Spectrochimica Acta Part B: Atomic Spectroscopy. 106: 36-44

Eggins SM, Kinsley LPJ, Shelley JMG (1998) Deposition and element fractionation processes during atmospheric pressure laser sampling for analysis by ICP-MS. Appl. Surf. Sci. 127-129: 278-286

Fan C, Zhan XC, Zeng P, Hu MY (2015) Multi-element Content Analysis of Rare Earth Fluorocarbonates from Bayan Obo Deposit by Laser Ablation-Inductively Coupled Plasma-Mass Spectrometry. Rock and Mineral Analysis. 34(6): 609-616

Fietzke J, Frische M (2016) Experimental evaluation of elemental behavior during LA-ICP-MS: influences of plasma conditions and limits of plasma robustness. J. Anal. At. Spectrom. 31(1): 234-244

Fricker MB, Kutscher D, Aeschlimann B, Frommer J, Dietiker R, Bettmer J, Günther D (2011) High spatial resolution trace element analysis by LA-ICP-MS using a novel ablation cell for multiple or large samples. International Journal of Mass Spectrometry. 307(1): 39-45

Fryer BJ, Jackson SE, Longerich HP (1995) The design, operation and role of the laser-ablation microprobe coupled with an inductively coupled plasma-mass spectrometer (LAM-ICP-MS) in the earth sciences. Canadian Mineralogist. 33: 303-312 
Garbe-Schönberg D, Müller S (2014) Nano-particulate pressed powder tablets for LA-ICP-MS. J. Anal. At. Spectrom. 29 (6): 990-1000

Garcia CC, Lindner H, Niemax K (2007) Transport efficiency in femtosecond laser ablation inductively coupled plasma mass spectrometry applying ablation cells with short and long washout times. Spectrochimica Acta Part B: Atomic Spectroscopy. 62(1): 13-19

Gerdes A, Zeh A (2009) Zircon formation versus zircon alteration - New insights from combined $\mathrm{U}-\mathrm{Pb}$ and $\mathrm{Lu}-\mathrm{Hf}$ in-situ LA-ICP-MS analyses, and consequences for the interpretation of Archean zircon from the Central Zone of the Limpopo Belt. Chem. Geol. 261(3): $230-243$

Guillong M, Heinrich CA (2007) Sensitivity enhancement in laser ablation ICP-MS using small amounts of hydrogen in the carrier gas. J. Anal. At. Spectrom. 22(12): 1488-1494

Guillong M, Horn I, Günther D (2003) A comparison of 266 nm, $213 \mathrm{~nm}$ and $193 \mathrm{~nm}$ produced from a single solid state Nd:YAG laser for laser ablation ICP-MS. J. Anal. At. Spectrom. 18(10): 1224-1230

Ho K-S, Lee W-W, Chan W-T (2015) Effects of ionization potential of an element and boiling point of the corresponding oxide on the sensitivity of ICP-MS. J. Anal. At. Spectrom. 30(10): 2066-2073

Hu ZC, Gao S, Liu YS, Hu SH, Chen HH, Yuan HL (2008) Signal enhancement in laser ablation ICP-MS by addition of nitrogen in the central channel gas. J. Anal. At. Spectrom. 23(8): 1093-1101 
Hu ZC, Liu YS, Chen L, Zhou LA, Li M, Zong KQ, Zhu LY, Gao S (2011) Contrasting matrix induced elemental fractionation in NIST SRM and rock glasses during laser ablation ICP-MS analysis at high spatial resolution. J. Anal. At. Spectrom. 26(2): 425-430

J. G. Mank A, R. D. Mason P (1999) A critical assessment of laser ablation ICP-MS as an analytical tool for depth analysis in silica-based glass samples. J. Anal. At. Spectrom. 14(8): $1143-1153$

Jochum KP, Stoll B, Herwig K, Willbold M, Hofmann AW, Amini M, Aarburg S, Abouchami W, Hellebrand E, Mocek B, Raczek I, Stracke A, Alard O, Bouman C, Becker S, Dücking M, Brätz H, Klemd R, de Bruin D, Canil D, Cornell D, de Hoog C-J, Dalpé C, Danyushevsky L, Eisenhauer A, Gao Y, Snow JE, Groschopf N, Günther D, Latkoczy C, Guillong M, Hauri EH, Höfer HE, Lahaye Y, Horz K, Jacob DE, Kasemann SA, Kent AJR, Ludwig T, Zack T, Mason PRD, Meixner A, Rosner M, Misawa K, Nash BP, Pfänder J, Premo WR, Sun WD, Tiepolo M, Vannucci R, Vennemann T, Wayne D, Woodhead JD (2006) MPI-DING reference glasses for in situ microanalysis: New reference values for element concentrations and isotope ratios. Geochemistry, Geophysics, Geosystems. 7(2): 1-44

Jochum KP, Stoll B, Weis U, Jacob DE, Mertz-Kraus R, Andreae MO (2014) Non-Matrix-Matched Calibration for the Multi-Element Analysis of Geological and Environmental Samples Using $200 \mathrm{~nm}$ Femtosecond LA-ICP-MS: A Comparison with Nanosecond Lasers. Geostand. Geoanal. Res. 38(3): 265-292

Jochum KP, Weis U, Stoll B, Kuzmin D, Yang QC, Raczek I, Jacob DE, Stracke A, Birbaum K, Frick DA, Günther D, Enzweiler J (2011) Determination of Reference Values for NIST SRM 610-617 Glasses Following ISO Guidelines. Geostand. Geoanal. Res. 35(4): 397-429

Koch J, Günther D (2011) Review of the State-of-the-Art of Laser Ablation Inductively Coupled Plasma Mass Spectrometry. Appl. Spectrosc. 65(5): 155A-162A 
Koch J, Wälle M, Dietiker R, Günther D (2008) Analysis of Laser-Produced Aerosols by Inductively Coupled Plasma Mass Spectrometry: Transport Phenomena and Elemental Fractionation. Analytical Chemistry. 80(4): 915-921

Kroslakova I, Günther D (2007) Elemental fractionation in laser ablation-inductively coupled plasma-mass spectrometry: evidence for mass load induced matrix effects in the ICP during ablation of a silicate glass. J. Anal. At. Spectrom. 22(1): 51-62

Kuhn H-R, Günther D (2003) Elemental fractionation studies in laser ablation inductively coupled plasma mass spectrometry on laser-induced brass aerosols. Analytical Chemistry. 75(4): $747-753$

Li Z, Hu Z, Liu Y, Gao S, Li M, Zong K, Chen H, Hu S (2015) Accurate determination of elements in silicate glass by nanosecond and femtosecond laser ablation ICP-MS at high spatial resolution. Chem. Geol. 400: 11-23

Liu YS, Hu ZC, Li M, Gao S (2013) Applications of LA-ICP-MS in the elemental analyses of geological samples. Chinese Science Bulletin. 58(32): 3863-3878

Longerich H, Günther D, Jackson S (1996) Elemental fractionation in laser ablation inductively coupled plasma mass spectrometry. Fresenius' journal of analytical chemistry. 355(5): 538-542

Luo T, Wang Y, Hu Z, Günther D, Liu Y, Gao S, Li M, Hu SH (2015) Further investigation into ICP-induced elemental fractionation in LA-ICP-MS using a local aerosol extraction strategy. J. Anal. At. Spectrom. 30(4): 941-949

Müller W, Shelley M, Miller P, Broude S (2009) Initial performance metrics of a new custom-designed ArF excimer LA-ICPMS system coupled to a two-volume laser-ablation cell. 
J. Anal. At. Spectrom. 24(2): 209-214

Machida R, Nakazawa T, Sakuraba Y, Fujiwara M, Furuta N (2015) Particle size-related elemental fractionation in laser ablation in liquid inductively coupled plasma mass spectrometry. J. Anal. At. Spectrom. 30(12): 2412-2419

Macholdt DS, Jochum KP, Pöhlker C, Stoll B, Weis U, Weber B, Müller M, Kappl M, Buhre S, Kilcoyne ALD, Weigand M, Scholz D, Al-Amri AM, Andreae MO (2015) Microanalytical methods for in-situ high-resolution analysis of rock varnish at the micrometer to nanometer scale. Chem. Geol. 411: 57-68

Sanborn M, Telmer K (2003) The spatial resolution of LA-ICP-MS line scans across heterogeneous materials such as fish otoliths and zoned minerals. J. Anal. At. Spectrom. 18(10): 1231-1237

Selby D, Creaser RA (2004) Macroscale NTIMS and microscale LA-MC-ICP-MS Re-Os isotopic analysis of molybdenite: Testing spatial restrictions for reliable Re-Os age determinations, and implications for the decoupling of Re and Os within molybdenite. Geochim. Cosmochim. Acta. 68(19): 3897-3908

Tomlinson EL, Thordarson T, Müller W, Thirlwall M, Menzies MA (2010) Microanalysis of tephra by LA-ICP-MS - Strategies, advantages and limitations assessed using the Thorsmörk ignimbrite (Southern Iceland). Chem. Geol. 279(3): 73-89

Ubide T, McKenna CA, Chew DM, Kamber BS (2015) High-resolution LA-ICP-MS trace element mapping of igneous minerals: In search of magma histories. Chem. Geol. 409: $157-168$

Yuan J, Zhan X, Hu M, Zhao L, Sun D (2015) Characterization of Matrix Effects in Microanalysis of Sulfide Minerals by Laser Ablation-Inductively Coupled Plasma-Mass 
Chapter 4.1 Elemental Fractionation Studies of 193 nm ArF Excimer Laser Ablation System at High Spatial Resolution

Spectrometry Based on An Element Pair Method. Spectroscopy and Spectral Analysis. 35(2): $512-518$

Yuan J, Zhan X, Sun D, Zhao L, Fan C, Kuai L, Hu M (2011) Investigationon Matrix Effects in Silicate Minerals by Laser Ablation-Inductively Coupled Plasma-Mass Spectrometry. Chinese Journal of Analytical Chemistry. 39(10): 1582-1588

Yuan J, Zhan X, Sun D, Zhao L, Fan C, Kuai L, Hu M (2012) Quantitative Analysis of Sulfide Minerals by Laser Ablation-Inductively Coupled Plasma-Mass Spectrometry Using Glass Reference Materials with Matrix Normalization Plus Sulfur Internal Standardization Calibration. Chinese Journal of Analytical Chemistry. 40(2): 201-207

Zhang S, He M, Yin Z, Zhu E, Hang W, Huang B (2016) Elemental fractionation and matrix effects in laser sampling based spectrometry. J. Anal. At. Spectrom. 31: 358-382 


\section{Chapter 4.2 Ablation Behaviors of 193nm ArF Excimer Laser for}

\section{Selected Sample Substrates}

Shitou $\mathrm{Wu}^{1}$,Chunxue $\mathrm{Xu}^{2 *}$, Klaus Simon ${ }^{1}$, Yilin Xiao ${ }^{3}$

(1.Geowissenschaftliches Zentrum, Göttingen Universität, Göttingen 37077, Germany;

2. National Research Center for Geoanalysis, Beijing 100037, China

2. School of Earth and Space Sciences, University of Science and Technology of China, Hefei Anhui 230026, China)

\section{Graphical abstract}

Ablation behaviors of 193 ArF excimer laser for silicate glasses, common minerals, and powder pellets were systematical investigated; Glasses and most of minerals have the controllable ablation behaviors, except quartz; Powder pellets have worse ablation behaviors, while their ablation behaviors could be improved either by increasing the tableting pressure or by decreasing the particle grain size; Ablation rate data of 43 different sample substrates were presented.

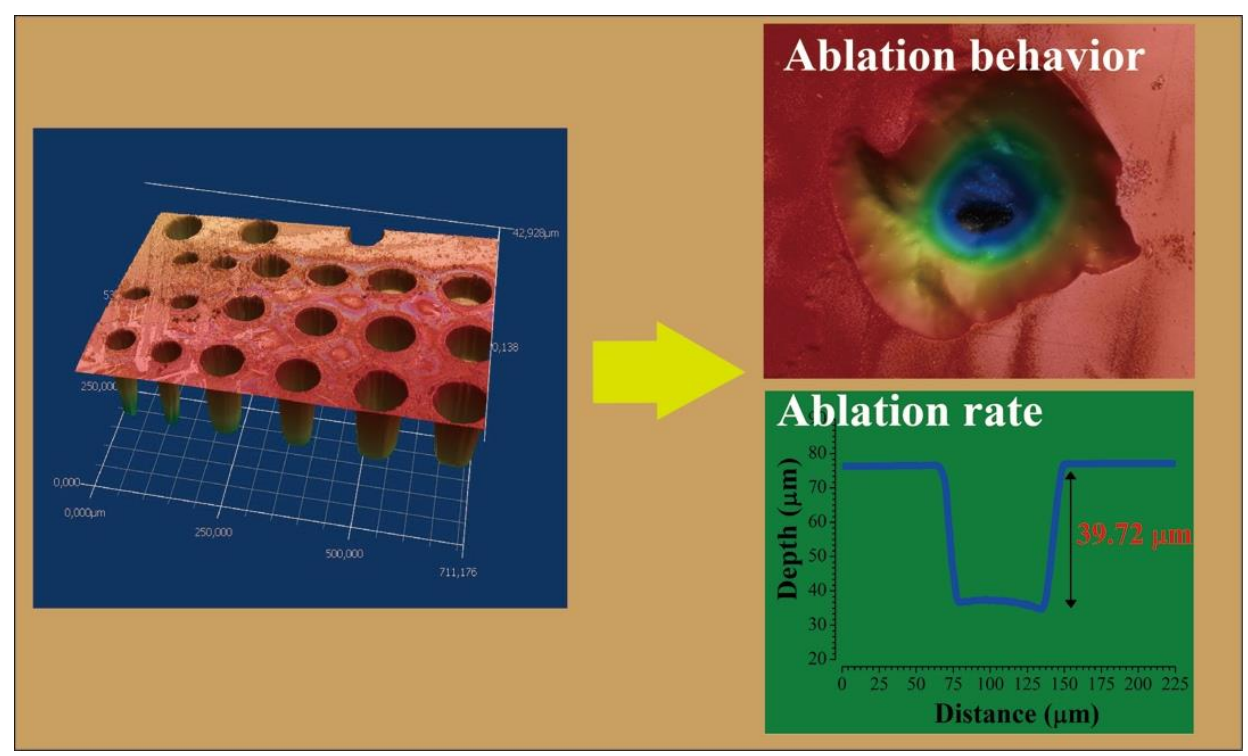




\begin{abstract}
Knowing laser ablation behaviors of different target materials is essential for selecting optimum laser parameters, choosing external reference materials, as well as for the assurance of data quality. In this study, ablation behaviors of 193 ArF excimer laser for silicate glasses, common minerals, and powder pellets were investigated. Ablation rates influenced by laser parameters (including spot size, energy density, and laser frequency) were evaluated. Topographic images of laser craters illustrate that glasses and most minerals have the controllable ablation behaviors, except quartz. The worse ablation behavior of quartz may be ascribed to the micro-fluid inclusions, which could result in the overheating effect in laser pits. Powder pellets have worse ablation behaviors compared to glass, while their ablation behaviors could be improved either by increasing the tableting pressure or by decreasing the particle grain size. Ablation rates gradually decrease when the ablation depth is 1.5 times larger than spot size. The maximum ablation depth could reach twice as much spot size at the experiment conditions (RESOlution M-50 laser system and energy density $3.0 \mathrm{~J} / \mathrm{cm}^{2}$ ). Ablation rates increase with the growth of laser energy density, while are not affected by the laser frequency $(2-20 \mathrm{~Hz})$. Ablation rates are specified to the individual sample substrates. In general, ablation rates of powder pellets are larger than glasses and minerals, carbonates and sulfides are greater than silicate minerals, NIST glasses are higher than geological glasses. Ablation rate data of 43 different sample substrates were presented, and these data could provide the reference for other laboratories.
\end{abstract}

Keywords: 193nm ArF laser; ablation behavior; ablation rate; energy density 


\subsubsection{Introduction}

Laser ablation-inductively coupled plasma-mass spectrometry (LA-ICP-MS) has been widely used for elemental- and isotopic-specific analyses in geochemistry (Liu et al., 2013; Russo et al., 2013), such as mineral microanalysis (Chew et al., 2014; Flem et al., 2002; Li et al., 2016; Stead et al., 2017), geochronology study (Li et al., 2016; Yang et al., 2014; Yuan et al., 2004; Zack et al., 2011), geothermal research (Audétat et al., 2015; Cruz - Uribe et al., 2016) as well as rock bulk analysis (He et al., 2016; Peters and Pettke, 2017). In the past decades, scientific questions associated with elemental fractionation (Hu et al., 2011; Tang et al., 2015; Wu et al., 2016), matrix effect (Jochum et al., 2014; Sylvester, 2008), quantification strategy (Jackson, 2008; Liu et al., 2008; Paton et al., 2010) as well as the development of reference materials (Audétat et al.,, 2015; Jochum et al., 2016; Klemme et al., 2008; Tabersky et al., 2014; Wu et al., 2015; Yang et al., 2012) have been investigated thoroughly, and significant improvement has been achieved. These studies promote the development as well as the application of LA-ICP-MS to a great extent. Laser ablation is one of the crucial components for this technique. A previous study (Horn et al., 2001) illustrated that the ablation behaviors are specified to the individual sample substrates. Investigating ablation behaviors for different substrates is of great significance (Borisov et al., 2000), such as (1) understanding the mechanism of elemental fractionation (Mank and Mason, 1999), (2) correction of ablation mass amount during each analysis, (3inter-laboratory comparison (Horstwood et al., 2016; Li et al., 2015), (4) applications in depth profile analysis (Horn et al.,, 2001), direct analysis in thin sections and high spatial analysis (Wu et al., 2016).

Ablation behaviors of some substrates using different wavelength laser (193nm, 213nm, 266nm) have been investigated in previous studies Günther and Heinrich (1999) studied the ablation behaviors of $193 \mathrm{~nm}$ and $266 \mathrm{~nm}$ lasers for NIST612 and demonstrated that ablation rates were nearly identical for both laser system at similar energy density, meanwhile illustrated that the aerosols ablated with $193 \mathrm{~nm}$ laser are finer than $266 \mathrm{~nm}$ laser. Jeffries et al. (1998) compared the ablation behaviors of $213 \mathrm{~nm}$ and $266 \mathrm{~nm}$ lasers and illustrated that 
ablation process is more controllable with a shorter wavelength laser. Borisov et al. (2000) investigated the ablation rates of a $266 \mathrm{~nm}$ laser which is influenced by the energy density and illustrated that ablation rates changed with the increase of energy density. Horn et al. (2001) reported the ablation rates of $193 \mathrm{~nm}$ and $266 \mathrm{~nm}$ lasers for NIST glasses and several metals. Kuhn et al. (2010) studied the ablation behaviors of NIST610 and 91500 (zircon reference material) using a $193 \mathrm{~nm}$ laser. The results illustrated that ablation rate of NIST610 is 1.5 times larger than zircon. The target materials investigated in previous studies are mainly NIST glasses, metals and zircon, and little data has been published for the substrates that include geological glasses, common minerals, and powder pellets. Those substrates are the common target materials for LA-ICP-MS in nowadays.

193nm ArF excimer laser provides advantages including high stability of output energy, high absorption efficiency and easy to maintain, and currently are widely installed in LA-ICP-MS laboratories. Therefore investigating ablation behaviors of $193 \mathrm{~nm}$ laser is of great significance. In this study, the ablation behaviors of $193 \mathrm{~nm}$ ArF laser for synthetic/geological glasses, common minerals, and powder pellets were investigated. Ablation behaviors were evaluated based on the topographic information of laser craters. Ablation rates influenced by laser parameters (including spot size, energy density, and laser frequency) were studied. The ablation rate data of 43 different individual substrates are presented.

\subsubsection{Experimental}

\subsubsection{Instrumentation}

The LA-ICP-MS and Laser Scanning Confocal Microscope (LSCM) were utilized for investigating ablation behaviors of $193 \mathrm{~nm}$ ArF laser. LA-ICP-MS was used for generating laser craters, and LSCM was employed for the collection of topographic images and the measurement of ablation depth. 


\subsection{LA-ICP-MS}

RESOlution M-50 ablation system (ASI, Australia) combined with an Element 2 sector field ICP-MS (ThermoScientific, USA) were used in this study. The operating parameters of LA-ICP-MS are summarized in Table 1. The laser was adjusted to the energy mode. Thus the output energy is constant. Due to the loss of laser energy during transmission in the optical path, we measured the energy density at the sample surface by using an energy measurement device (FieldMasIITM, Coherent Company, Germany). That can ensure the data accuracy of the laser energy density.

Table1 Operation conditions of LA-ICP-MS system

\begin{tabular}{|c|c|c|c|}
\hline \multicolumn{2}{|c|}{ LA-ICP-MS ablation system } & \multicolumn{2}{|c|}{ ICP-MS } \\
\hline Laser type & RESOlution M-50 ArF excimer & ICP-MS & Element 2 \\
\hline wavelength & $193 \mathrm{~nm}$ & RF power & $1500 \mathrm{~W}$ \\
\hline Pulse width & $20 \mathrm{~ns}$ & Guard electrode & Floated \\
\hline Energy density & $1.0 \sim 11.0 \mathrm{~J} / \mathrm{cm}^{2}$ & Coolant gas flow (Ar) & $15.00 \mathrm{~L} / \mathrm{min}$ \\
\hline Frequency & $2 \sim 20 \mathrm{~Hz}$ & Auxiliary gas flow (Ar) & $1.00 \mathrm{~L} / \mathrm{min}$ \\
\hline Ablation cell & Laurin Technic S-155 & Makeup gas flow (Ar) & $0.95 \mathrm{~L} / \mathrm{min}$ \\
\hline Spot size & $10 \sim 90 \mu \mathrm{m}$ & Segment duration & $10 \mathrm{~ms}$ \\
\hline Carrier gas flow (He) & $0.65 \mathrm{~L} / \mathrm{min}$ & Detector & Counting and analog \\
\hline Ablation time & $20 \sim 120 \mathrm{~s}$ & Resolution (M/ $\Delta \mathrm{M})$ & Low $(\sim 300)$ \\
\hline
\end{tabular}

\subsection{Laser Scanning Confocal Microscope}

Topographic images of laser craters were collected using the VK-X200 series Laser Scanning Confocal Microscope (Keyence Company, Germany). The LSCM configured with a $408 \mathrm{~nm}$ ultraviolet laser and a 16-bit photomultiplier and four objective lenses including $10 \times, 20 \times$, $50 \times$ and $150 \times$. All the topographic images were collected using $10 \times$ and $20 \times$ lenses. The topographic images were processed using the instrument software (MultiFileAnalyzer), which includes the visualization of topographic images and the measurement of ablation 
depth. The assessment process of a laser crater is shown in Fig.1. Figure 1 illustrates that the diameter of laser crater became smaller with the growth of ablation depth. The crater shape is like an inverted cone, which is probably caused by the laser dynamic defocusing with the growth of ablation depth (Horn et al., 2001).

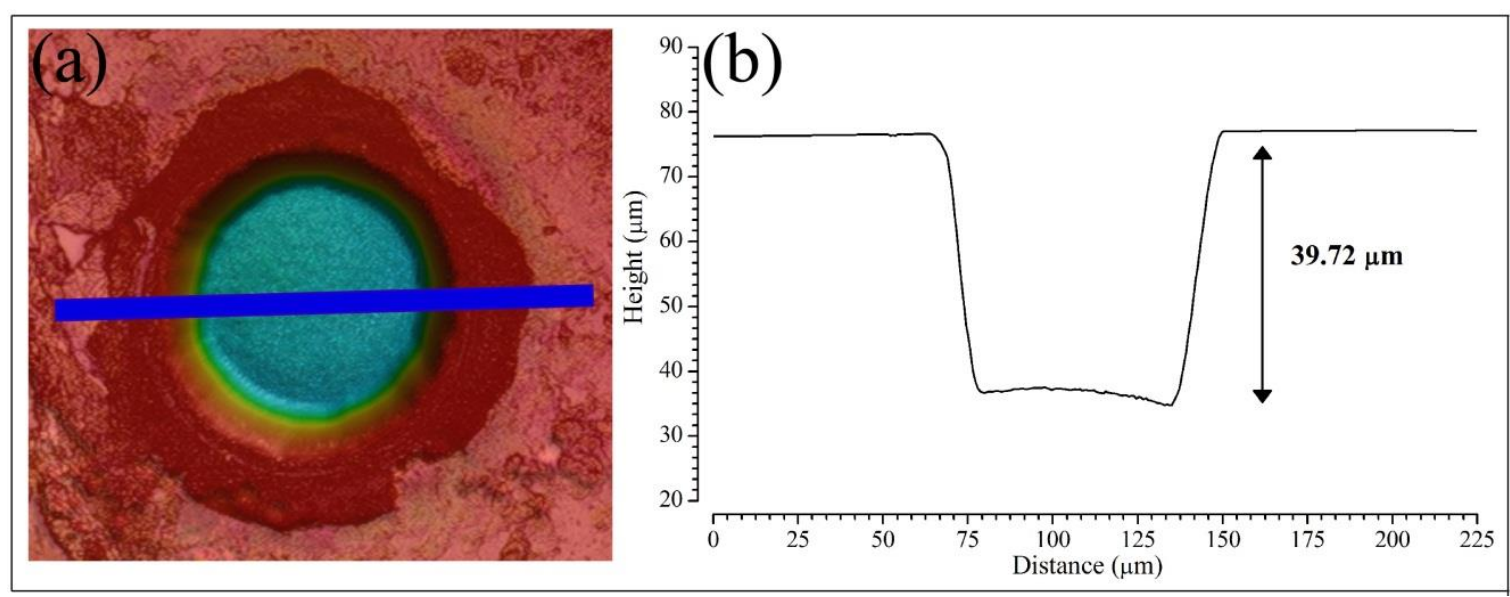

Fig.1 Assessment process of a laser crater generated during ablation procedure based on Laser Scanning Confocal Microscope. (a) 3D topographic image of a laser crater. (b) Trace of the topography along the line indicated in the 3D image. The material shown here is NIST 610.

\subsubsection{Samples}

The target materials for LA-ICP-MS mainly include synthetic/geological glasses, common minerals, and powder pellets. In this study, ablation behaviors of 43 individual sample substrates were investigated. That includes eighteen synthetic/geological glasses, twenty common minerals and five powder pellets. The synthetic/geological glasses are NIST, MPI-DING, UGSG, and CGSG glass reference materials.

Synthetic glass: NIST610, NIST612, NIST614, and GSD-1G;

Geological glass: StHs6/80-G, ATHO-G, T1-G, ML3B-G, KL2-G, GOR128-G, GOR132-G, BCR-2G, BHVO-2G, BIR-1G, CGSG-1, CGSG-2, CGSG-4, and CGSG-5;

Common mineral: Feldspar (plagioclase and sanidine), Garnet, Epidote, Scapolite, Amphibole, Zircon (91500, CJ-1 and Plešovice), Mica (biotite and muscovite), Sodalite, 
Ilmenite, Pyroxene, Chlorite, Olivine, Hematite, Apatite, Calcite, Stalagmite, Serpentine, Limestone, Pyrite and Quartz (Audétat et al., 2015);

Powder pellets: GBW07130, G1RF-85a, MACS-3, MASS-1, PB40-1.

Powder pellet GBW07130 was tableted at the pressure of $160 \mathrm{t}$, and the matrix powder is the powdered marble reference material GBW07130. G1RF-85a and PB40-1 are in-house powder standards. The matrixes are granite and picrate, respectively. The powders were milled to nanoscale $\left(d_{50}: 730 \mathrm{~nm}\right)$ by using a wet milling protocol, and then pressed to pellets at the pressure of $20 \mathrm{t}$. The detailed wet milling protocol is shown in the literature (Garbe-Schönberg and Müller, 2014).

\subsubsection{Results and discussion}

\subsubsection{Topographic images of laser craters}

The topographic information of laser generated cavities could be used for interpreting the quality of LA-ICP-MS data. Zhang et al. (2016) reported that the analytical precision of powder pellets was worse than glasses, which may be related to the less controllable ablation for powder pellets. In this study, the topographic images of laser craters in selected substrates were evaluated. The substrates include synthetic glass (NIST610), geological glass (BCR-2G), apatite, pyrite, quartz, and three powder pellets (MACS-3, GBW07130, and PB40-1). The topographic images are shown in Fig.2. 

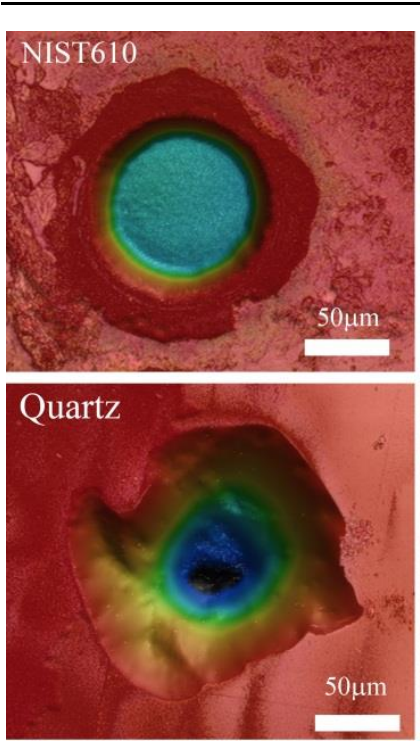
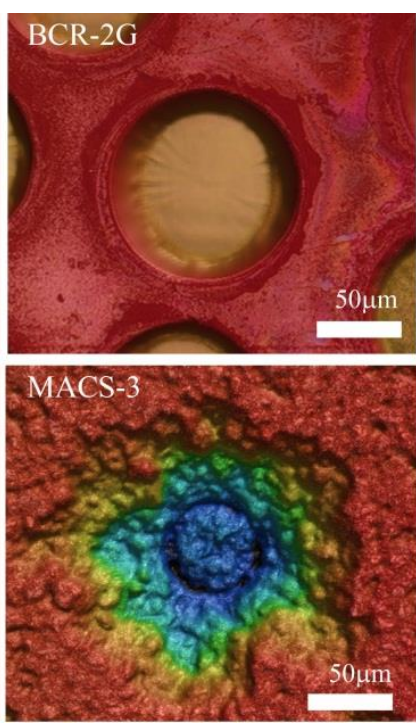
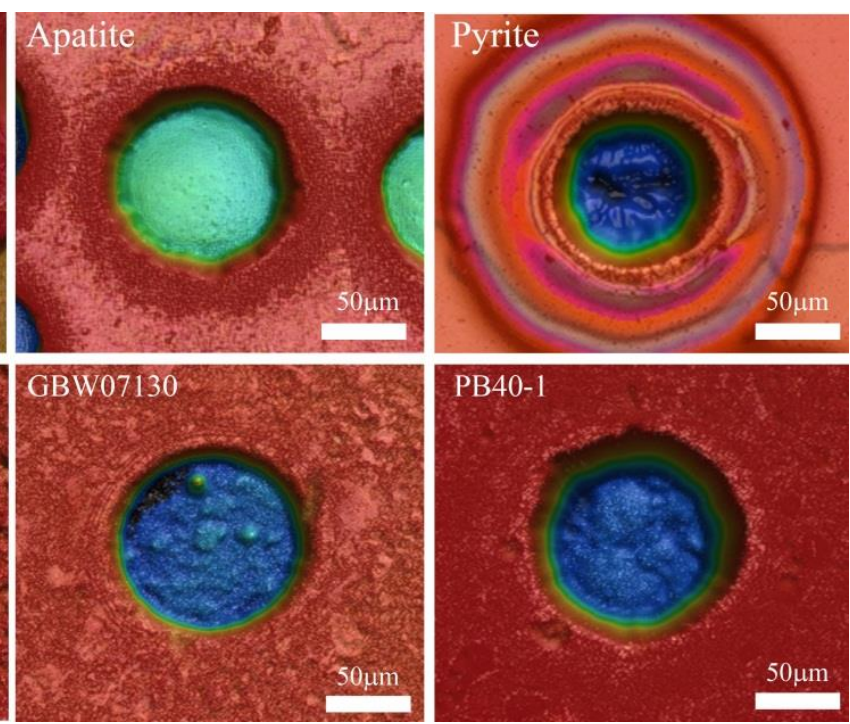

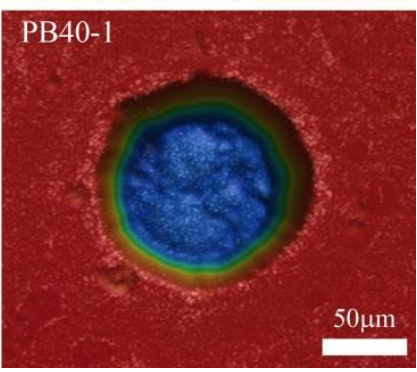

Fig. 2 Topographic images of laser craters in NIST610, BCR-2G, Apatite, Pyrite, Quartz,

MACS-3, GBW01730, and PB40-1. Ablation conditions are energy density $5.0 \mathrm{~J} \mathrm{~cm}^{-2}$ and spot size $75 \mu \mathrm{m}$.

Figure 2 illustrates that crater shapes of NIST610, BCR-2G, and Apatite are the well-regulated circles, which reveals that these three substrates have controllable ablation under $193 \mathrm{~nm}$ ArF laser. Crater edge of pyrite is slightly deformed, and the halo effect is significant, and melting effects occurred. This phenomenon could be interpreted by the less thermal conductivity of pyrite. Crater of quartz presents irregular as water chestnut, which infers the occurrence of splashing ablation. The splashing ablation may be caused by the micro-fluid inclusions, which could result in the overheating effect in laser pits. Jeffries et al. (1998) investigated the ablation behaviors of substrates which have less photon absorption (e.g. quartz and $\mathrm{MgF}_{2}$ ) and reported that these substrates had worse ablation behaviors. The splashing ablation occurred during ablating quartz may be caused by less photon absorption of quartz. MACS-3 has uncontrolled ablation behavior, which is related to the less adhesion of powder pellets. Zhang et al. (2016) illustrated that the analytical precision of powder pellets was worse than glasses. Contrarily, ablations of GBW07130 and PB40-1 are well controlled. GBW07130 was pressed at the pressure of 160 t. PB40-1 was nanoparticle powder pellet. The adhesion of powder pellets could be enhanced by increasing the tableting pressure or decreasing the particle grain size, thus improves the ablation behaviors 
(Garbe-Schönberg and Müller, 2014; Peters and Pettke, 2017). In summary, 193nm laser ablations of glasses and most minerals are well controlled, but not for quartz and powder pellets (standard powder pellets). However, ablation behaviors of powder pellets could be improved by increasing the tableting pressure or decreasing the particle grain size.

\subsubsection{Ablation rates influenced by laser parameters}

Ablation rates are highly influenced by the laser parameters (including spot size, energy density, and laser frequency). Here, we investigated the effect of spot size, energy density, and laser frequency.

\subsubsection{Ablation rates affected by spot size}

Recent years, more attention has been paid to high spatial resolution analysis using LA-ICP-MS (Wu et al., 2016), such as elemental mapping (Raimondo et al., 2017; Ubide et al., 2015), and the knowledge of ablation rates influenced by spot size could provide crucial information for these applications. In this study, the effects of spot size on the ablation rates were investigated. NIST610 was used as the subject sample. The results are illustrated in Fig.3.

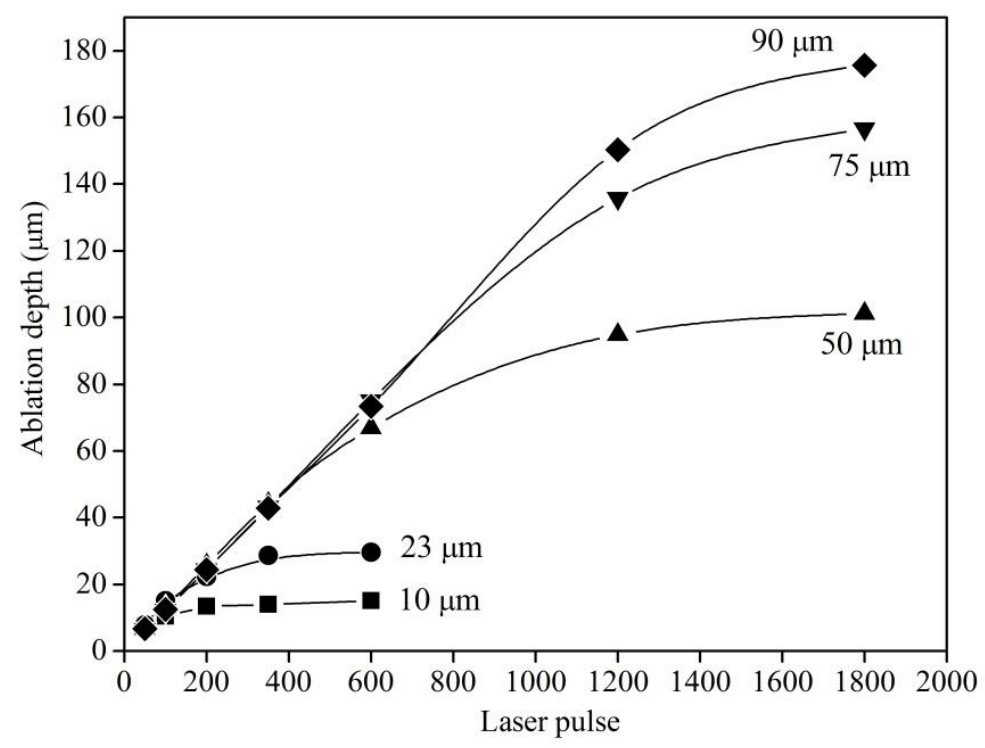

Fig. 3 The relationship of ablation depth and laser pulses at different laser spot size. Laser ablation conditions are energy density $3.0 \mathrm{~J} / \mathrm{cm}^{2}$ and laser frequency $10 \mathrm{~Hz}$. 
Figure 3 illustrates that when ablation depth is smaller than 1.5 times of spot size (ablation depth: spot size < 1.0: 1.5), the ablation depth is linearly increased with the laser pulse. The increment of ablation depth gradually decreases with laser pulse when the ablation depth is larger than 1.5 times of spot size, which demonstrates the declining of ablation rates. The ablation depth is kept constant as the ablation depth around twice as much spot size, and that illustrates that the ablation rate is zero. The decrease of ablation rates (when ablation depth is 1.5 times larger than spot size) might be ascribed to the laser dynamic defocusing (Bi et al., 2000). The laser is focused on the sample surface in this study. With the growth of ablation depth, the laser may be defocused. The defocused laser could lead to a less photon absorption, thus resulting in the decrease of ablation rates. Mank and Mason (1999) reported the ratio of ablation depth and spot size could be six, while our results illustrate that the maximum ablation depth is twice as much spot size at the experiment conditions (RESOlution M-50 laser system and the energy density $3.0 \mathrm{~J} / \mathrm{cm}^{2}$ ). The different laser systems could interpret the inconsistency. The optical path and the focusing device may highly affect the maximum ablation depth (Mank and Mason, 1999; Müller et al., 2009). Considering the results of this study, a specific optimization for the ablation time with a given spot size is a prerequisite for the application of LA-ICP-MS at high spatial resolution (spot size $<15 \mu \mathrm{m}$ ).

\subsubsection{Ablation rates influenced by energy density and laser frequency}

Energy density and laser frequency are the critical parameters for LA-ICP-MS, especially for the application in depth profile analysis (Steely et al., 2014). The energy density is one of the parameters affecting the elemental fractionation (Gaboardi and Humayun, 2009; Jackson and Gunther, 2003). In this study, ablation rates affected by the energy density and laser frequency were investigated based on five substrates (including synthetic glass NIST610, geological glass BCR-2G, apatite, calcite, and amphibole). The results are shown in Fig.4. 

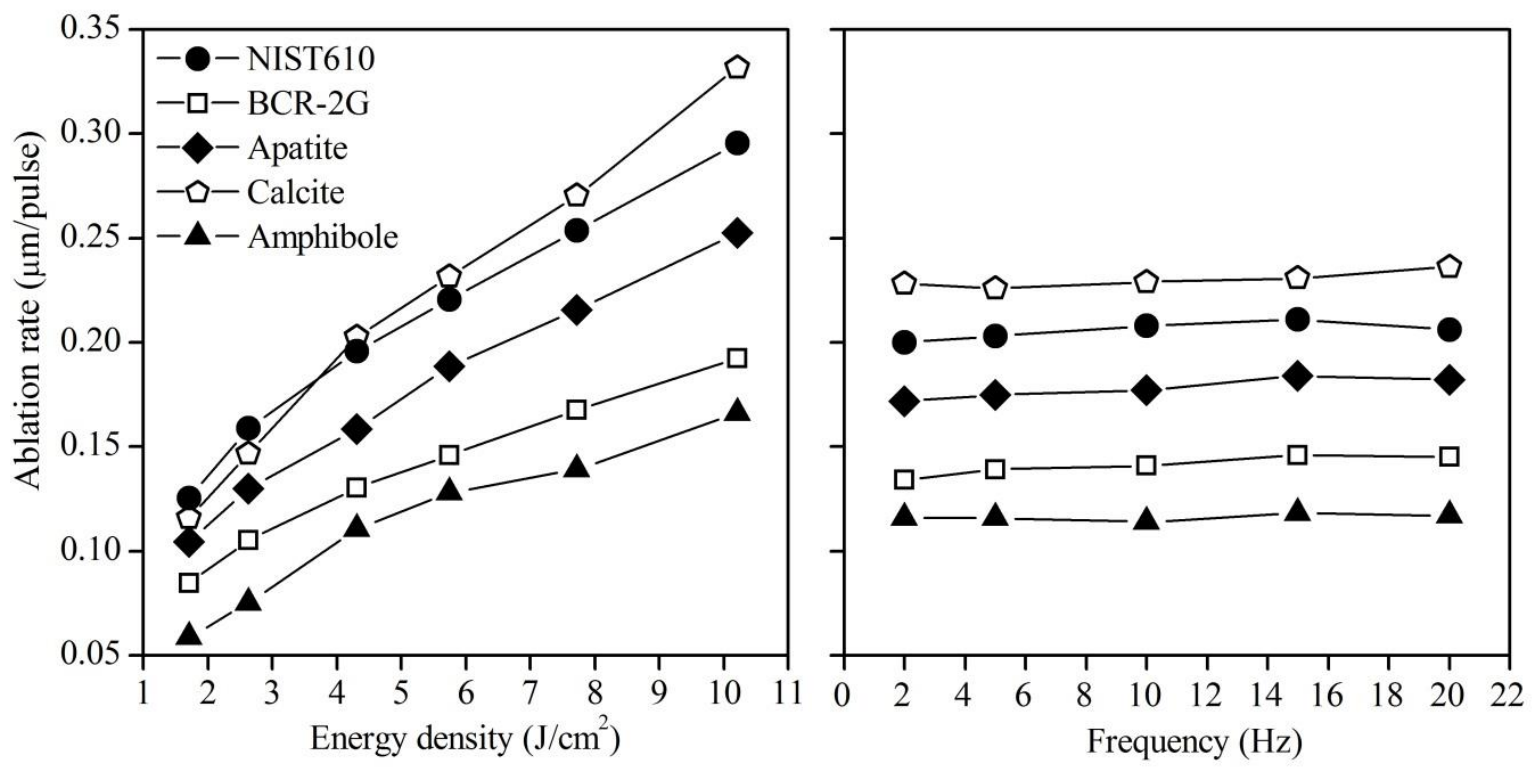

Fig. 4 Variations of ablation rates with changing energy density and laser frequency. For the energy density experiment, the laser parameters are set as laser frequency $(5 \mathrm{~Hz})$, spot size (75 $\mu \mathrm{m})$, and ablation time (20s). For the laser frequency experiment, the laser parameters are energy density $\left(4.2 \mathrm{~J} \mathrm{~cm}^{-2}\right)$, spot size $(75 \mu \mathrm{m})$, and laser pulses (150).

The results illustrate that the ablation rates increase with the growth of energy density. The increments of ablation rates are specific to individual substrates. The ablation rates of NIST610, Calcite, and Amphibole grow faster when the energy density is smaller than $4.0 \mathrm{~J}$ $\mathrm{cm}^{-2}$ and increased slower when the energy density is larger than $4.0 \mathrm{~J} \mathrm{~cm}^{-2}$. However, ablation rates of BCR-2G and Apatite are linearly increased with energy density. Mao et al. (Mao and Russo, 1996) illustrated that with the growth of energy density, plasma shielding effect occurred in laser pits, which could result in less photon absorption. For NIST610, Calcite, and Amphibole, the plasma shielding effect may occur at an energy density of 4.0 $\mathrm{J} / \mathrm{cm}^{2}$. Thus the growth of ablation rates turns to be less. The plasma shielding effects may be affected by the sample matrix, and that could interpret that the increase of ablation rates of BCR-2G and Apatite is nearly constant. Russo et al. (Russo et al., 2004) reported that the mechanism of ablation process changed with the increase of energy density, thus affecting the ablation rates. Laser frequency could also affect the plasma generated during the ablation, 
and modify the ablation rates. While the data (Fig.4) illustrate that the ablation rates are constant at the laser frequency in the range of $2-20 \mathrm{~Hz}$, which demonstrates that the laser frequency does not influence the ablation rates.

\subsection{Ablation rates of target materials}

Ablation rates are influenced by the optical and physicochemical properties of sample substrates, which include photon absorption efficiency, hardness, and density. In this study, a total of 43 different substrates including synthetic/geological glasses, common minerals, and powder pellets were investigated. The results are shown in Fig.5.

Figure 5 reveals that ablation rates of the investigated substrates are in a wide range from $0.055 \mu \mathrm{m} /$ pulse to $3.65 \mu \mathrm{m} /$ pulse. The discussions are spread out in three groups including synthetic/geological glass, common mineral, and powder pellet. It should be emphasized that due to the different laser setups (optical path and focus device) as well as the chemical compositions of glasses and minerals, ablation rates reported in this study may be inconsistent with other laboratories. 


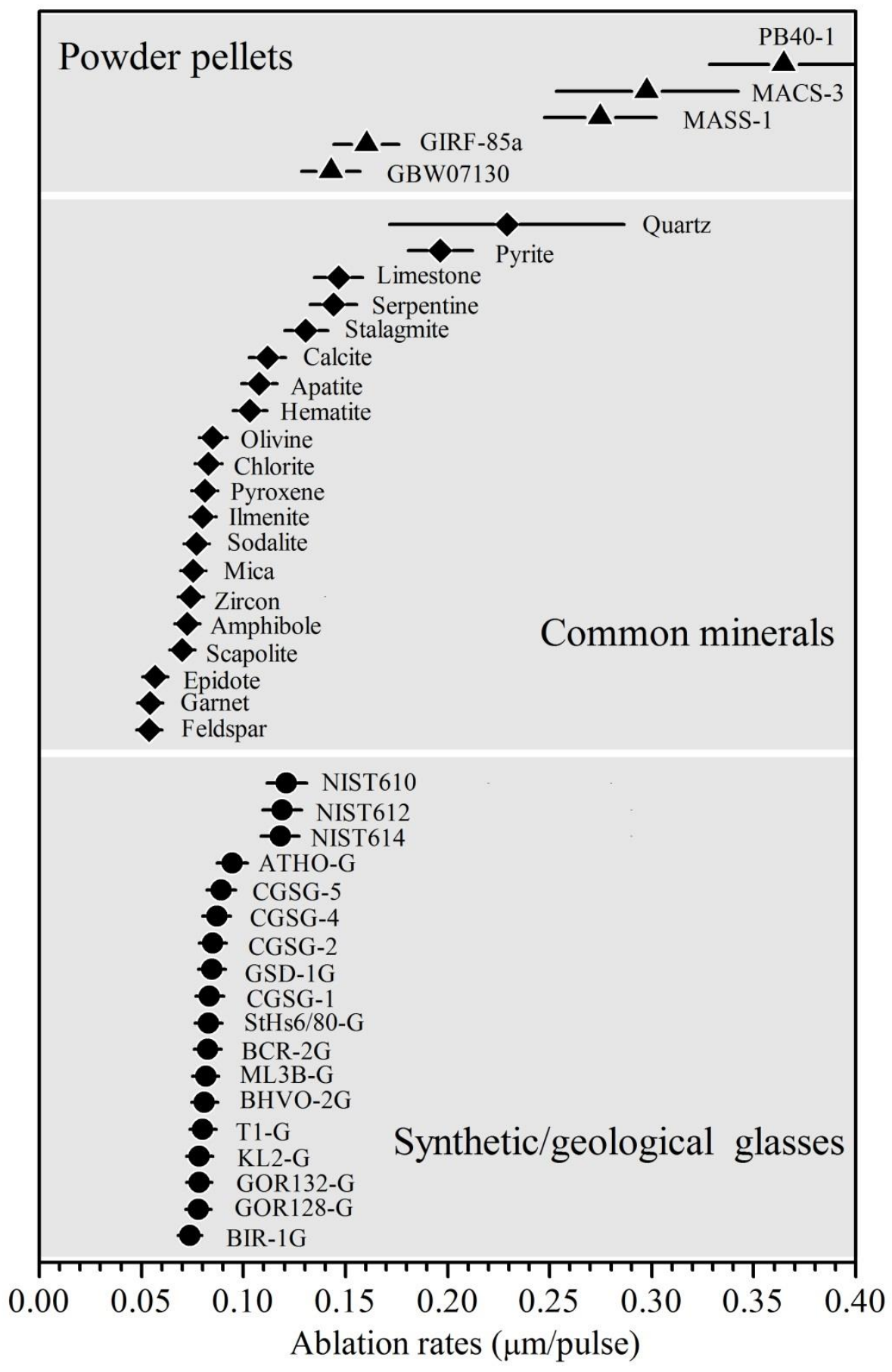

Fig. 5 Ablation rates of 43 individual substrates. The data are collected at the laser energy density $2.0 \mathrm{~J} \mathrm{~cm}^{-2}$ (expect quartz, $5.0 \mathrm{~J} \mathrm{~cm}^{-2}$ ). The error bars are derived from three analyses as one standard deviation. 


\subsubsection{Ablation rates of synthetic/geological glasses}

Figure 5 illustrates that different glasses have their specific ablation rates. The ablation rates of NIST glasses are similar and are apparently greater than geological glasses. Slight different ablation rates are observed among geological glasses. The similar ablation rates of NIST glasses (from dark blue NIST610 to light blue NIST612 to transparent NIST610) indicate that the photon absorption efficiency of $193 \mathrm{~nm}$ ArF laser is not affected by the glass transparency. This observation is consistent with that reported by Horn et al. (2001). GSD-1G is also synthetic glass. However, the ablation rate is lower compared to NIST glasses, which demonstrates that the glass preparation method does not influence the ablation rate. Ablation rates may be controlled by the major components of glasses. The results illustrate that ablation rates of geological glasses are positively correlated with the $\mathrm{SiO}_{2}$ content. Glasses containing higher $\mathrm{SiO}_{2}$ content (like ATHO-G) have larger ablation rates. Other properties including quenching temperature, quenching time and density may also affect the ablation rates. Hu et al. (2011) reported the ablation rate of NIST610 is 1.5 times higher than GSE-1G. The factors influencing ablation rates need further investigations. In summary, ablation rates of NIST glasses are obviously greater than geological glasses. Thus there may be matrix effects between those two type glasses.

\subsubsection{Ablation rates of common minerals}

Figure 5 shows that different minerals have different ablation rates. Feldspar has the smallest ablation rate, and quartz has the highest ablation rate. The ablation rates of carbonates and sulfides are greater than silicate minerals. The factors affecting the ablation rates include the photon absorption efficiency, hardness, and density, etc. Minerals with higher density (like Garnet) and hardness (like Zircon) have relatively small ablation rates. The significant ablation rate of quartz may be ascribed to the splashing ablation effect. Currently, little data have been reported for the photon absorption efficiency of $193 \mathrm{~nm}$ laser for different substrates. Jeffries et al. (Jeffries et al.,, 1998) demonstrated that quartz has less photon 
absorption of $266 \mathrm{~nm}$ laser. Although the photon absorption of $266 \mathrm{~nm}$ laser and $193 \mathrm{~nm}$ laser may be different (Horn et al., 2001), the small ablation rate of Feldspar may be related to the low absorption efficiency. Meanwhile, the relatively low laser energy density (used in this study) may also be a factor leading the small ablation rate for Feldspar. Since the ablation rates are specified to individual minerals, the ablation mass amount during each analysis may be different. Thus an internal standard must be used when non-matrix-matched calibration is applied.

\subsubsection{Ablation rates of powder pellets}

Compared to glasses and minerals, the ablation rates of powder pellets are relatively large, which may be related to the less adhesion of powder pellets. Ablation rates of different powder pellets are also inconsistent. The powder pellets are formed based on the adhesion force between powder particles. More mechanical ablation is expected in the ablation process. Compared to MACS-3 (worse ablation behavior), the ablation rates of GBW07130 (160t pressure) and G1RF-85a (nano size particles) are relatively small. Figure 2 shows that the ablation behaviors could be improved by increasing tableting pressure or decreasing particle grain size. The less controllable ablation behavior may lead to the significant ablation rates. However, this interpretation could not explain the significant ablation rate of PB40-1. PB40-1 is the nanoparticle powder pellet, and the ablation behavior is well controlled. The relative large ablation rates may be ascribed to the high water content ( $8 \mathrm{wt} \%$ ), which may enhance the photon absorption. Due to the optical and physicochemical properties of powder pellets, the ablation rates of powder pellets are larger than glasses and minerals. Thereby severe matrix effects may exist between powder pellets and glass reference materials. 


\subsubsection{Conclusions}

In this study, the ablation behaviors of $193 \mathrm{~nm} \mathrm{ArF} \mathrm{laser} \mathrm{for} \mathrm{synthetic/geological} \mathrm{glasses,}$ common minerals and powder pellets were investigated. The ablation rates influenced by laser parameters including spot size, energy density, and laser frequency were studied. The topographic images of laser craters show that the ablation behavior of quartz is worse than other minerals. The micro-fluid inclusions in quartz may cause this uncontrolled ablation, which leads to the overheating effect in laser pits. The ablations of powder pellets are less controlled compared to that of glasses while the ablation behavior could be improved by either increasing the tableting pressure or decreasing the particle grain size. The ablation rates gradually decrease when the ablation depth is larger than 1.5 times of spot size. The ablation rates increase with the growth of laser energy density, while they are not affected by the laser frequency $(2-10 \mathrm{~Hz})$. The matrix effect, at least in ablation process, between NIST glasses and geological glasses was proved. The ablation rates of powder pellets are larger than glasses and minerals, carbonates and sulfides are greater than silicate minerals. We presented the ablation rates of 43 different substrates, which may provide a reference for other laboratories.

\section{Acknowledgments}

We thank Dr. Andreas Kronz, Dr. Burkhard Schmidt, Dr. Istvan Dunkl and Smruti Sourav Rout from Göttingen University, Dr. Chao Zhang from Hannover University, Prof. Dr. Yanping Wang, Prof. Dr. Xiuchun Zhan, Dr. Chunxue Xu from National Research Center of Geoanalysis, Haiyang Liu from University of Science and Technology of China, Huan Tian from China Unversity of Geosciences (Wuhan), and Lingqi Zeng from Chengdu University of Technology for the providing samples. 


\section{References}

Audétat A, Garbe-Schönberg D, Kronz A, Pettke T, Rusk B, Donovan JJ, Lowers HA (2015) Characterisation of a Natural Quartz Crystal as a Reference Material for Microanalytical Determination of Ti, Al, Li, Fe, Mn, Ga and Ge. Geostand. Geoanal. Res. 39(2): 171-184

Bi M, Ruiz AM, Gornushkin I, Smith BW, Winefordner JD (2000) Profiling of patterned metal layers by laser ablation inductively coupled plasma mass spectrometry (LA-ICP-MS). Appl. Surf. Sci. 158(3-4): 197-204

Borisov OV, Mao X, Russo RE (2000) Effects of crater development on fractionation and signal intensity during laser ablation inductively coupled plasma mass spectrometry. Spectrochimica Acta Part B: Atomic Spectroscopy. 55(11): 1693-1704

Chew DM, Donelick RA, Donelick MB, Kamber BS, Stock MJ (2014) Apatite Chlorine Concentration Measurements by LA-ICP-MS. Geostand. Geoanal. Res. 38(1): 23-35

Cruz-Uribe AM, Mertz-Kraus R, Zack T, Feineman MD, Woods G, Jacob DE (2016) A New LA-ICP-MS Method for Ti in Quartz: Implications and Application to High Pressure RutileQuartz Veins from the Czech Erzgebirge. Geostand. Geoanal. Res. 41(1): 29-40

Flem B, Larsen RB, Grimstvedt A, Mansfeld J (2002) In situ analysis of trace elements in quartz by using laser ablation inductively coupled plasma mass spectrometry. Chem. Geol. 182(2-4): $237-247$

Günther D, Heinrich CA (1999) Comparison of the ablation behaviour of $266 \mathrm{~nm} \mathrm{Nd:} \mathrm{YAG}$ and $193 \mathrm{~nm}$ ArF excimer lasers for LA-ICP-MS analysis. J. Anal. At. Spectrom. 14(9): $1369-1374$ 
Gaboardi M, Humayun M (2009) Elemental fractionation during LA-ICP-MS analysis of silicate glasses: implications for matrix-independent standardization. J. Anal. At. Spectrom. 24(9): 1188-1197

Garbe-Schönberg D, Müller S (2014) Nano-particulate pressed powder tablets for LA-ICP-MS. J. Anal. At. Spectrom. 29 (6): 990-1000

He Z, Huang F, Yu H, Xiao Y, Wang F, Li Q, Xia Y, Zhang X (2016) A Flux-Free Fusion Technique for Rapid Determination of Major and Trace Elements in Silicate Rocks by LA-ICP-MS. Geostand. Geoanal. Res. 40(1): 5-21

Horn I, Guillong M, Günther D (2001) Wavelength dependant ablation rates for metals and silicate glasses using homogenized laser beam profiles - implications for LA-ICP-MS. Appl. Surf. Sci. 182(1-2): 91-102

Horstwood MS, Košler J, Gehrels G, Jackson SE, McLean NM, Paton C, Pearson NJ, Sircombe K, Sylvester P, Vermeesch P (2016) Community-Derived Standards for LA-ICPMS U-(Th-) Pb Geochronology-Uncertainty Propagation, Age Interpretation and Data Reporting. Geostand. Geoanal. Res. 40(3): 311-332

Hu ZC, Liu YS, Chen L, Zhou LA, Li M, Zong KQ, Zhu LY, Gao S (2011) Contrasting matrix induced elemental fractionation in NIST SRM and rock glasses during laser ablation ICP-MS analysis at high spatial resolution. J. Anal. At. Spectrom. 26(2): 425-430

J. G. Mank A, R. D. Mason P (1999) A critical assessment of laser ablation ICP-MS as an analytical tool for depth analysis in silica-based glass samples. J. Anal. At. Spectrom. 14(8): $1143-1153$ 
Jackson SE (2008) Calibration strategies for elemental analysis by LA-ICP-MS. Laser Ablation ICP-MS in the Earth Sciences: Current Practices and Outstanding Issues (P. Sylvester, ed.). Mineralogical Association of Canada. Short Course Series. 40: 169-188

Jackson SE, Gunther D (2003) The nature and sources of laser induced isotopic fractionation in laser ablation-multicollector-inductively coupled plasma-mass spectrometry. J. Anal. At. Spectrom. 18(3): 205-212

Jeffries TE, Jackson SE, Longerich HP (1998) Application of a frequency quintupled Nd: YAG source $(\lambda=213 \mathrm{~nm})$ for laser ablation inductively coupled plasma mass spectrometric analysis of minerals. J. Anal. At. Spectrom. 13(9): 935-940

Jochum KP, Stoll B, Weis U, Jacob DE, Mertz-Kraus R, Andreae MO (2014) Non-Matrix-Matched Calibration for the Multi-Element Analysis of Geological and Environmental Samples Using $200 \mathrm{~nm}$ Femtosecond LA-ICP-MS: A Comparison with Nanosecond Lasers. Geostand. Geoanal. Res. 38(3): 265-292

Jochum KP, Wilson SA, Becker H, Garbe-Schönberg D, Groschopf N, Kadlag Y, Macholdt DS, Mertz-Kraus R, Otter LM, Stoll B, Stracke A, Weis U, Haug GH, Andreae MO (2016) FeMnOx-1: A new microanalytical reference material for the investigation of $\mathrm{Mn}-\mathrm{Fe}$ rich geological samples. Chem. Geol. 432: 34-40

Klemme S, Prowatke S, Münker C, Magee CW, Lahaye Y, Zack T, Kasemann SA, Cabato EJA, Kaeser B (2008) Synthesis and Preliminary Characterisation of New Silicate, Phosphate and Titanite Reference Glasses. Geostand. Geoanal. Res. 32(1): 39-54

Kuhn BK, Birbaum K, Luo Y, Günther D (2010) Fundamental studies on the ablation behaviour of $\mathrm{Pb} / \mathrm{U}$ in NIST 610 and zircon 91500 using laser ablation inductively coupled plasma mass spectrometry with respect to geochronology. J. Anal. At. Spectrom. 25(1): $21-27$ 
Li CY, Zhang RQ, Ding X, Ling MX, Fan WM, Sun WD (2016) Dating cassiterite using laser ablation ICP-MS. Ore Geology Reviews. 72: 313-322

Li X, Liu X, Liu Y, Su L, Sun W, Huang H, Yi K (2015) Accuracy of LA-ICPMS zircon $\mathrm{U}-\mathrm{Pb}$ age determination: An inter-laboratory comparison. Science China Earth Sciences. 58(10): 1722-1730

Li Z, Hu Z, Günther D, Zong K, Liu Y, Luo T, Zhang W, Gao S, Hu S (2016) Ablation Characteristic of Ilmenite using UV Nanosecond and Femtosecond Lasers: Implications for Non-Matrix-Matched Quantification. Geostand. Geoanal. Res. 40(4): 477-491

Liu YS, Hu ZC, Gao S, Gunther D, Xu J, Gao CG, Chen HH (2008) In situ analysis of major and trace elements of anhydrous minerals by LA-ICP-MS without applying an internal standard. Chem. Geol. 257(1-2): 34-43

Liu YS, Hu ZC, Li M, Gao S (2013) Applications of LA-ICP-MS in the elemental analyses of geological samples. Chinese Science Bulletin. 58(32): 3863-3878

Müller W, Shelley M, Miller P, Broude S (2009) Initial performance metrics of a new custom-designed ArF excimer LA-ICPMS system coupled to a two-volume laser-ablation cell. J. Anal. At. Spectrom. 24(2): 209-214

Mao X, Russo RE (1996) Invited paper Observation of plasma shielding by measuring transmitted and reflected laser pulse temporal profiles. Applied Physics A: Materials Science \& Processing. 64(1): 1-6

Paton C, Woodhead JD, Hellstrom JC, Hergt JM, Greig A, Maas R (2010) Improved laser ablation U-Pb zircon geochronology through robust downhole fractionation correction. Geochemistry, Geophysics, Geosystems. 11(3): 1-36 
Peters D, Pettke T (2017) Evaluation of Major to Ultra Trace Element Bulk Rock Chemical Analysis of Nanoparticulate Pressed Powder Pellets by LA-ICP-MS. Geostand. Geoanal. Res. 41(1): 5-28

Raimondo T, Payne J, Wade B, Lanari P, Clark C, Hand M (2017) Trace element mapping by LA-ICP-MS: assessing geochemical mobility in garnet. Contributions to Mineralogy and Petrology. 172(4): DOI 10.1007/s00410-00017-01339-z

Russo RE, Mao X, Gonzalez JJ, Zorba V, Yoo J (2013) Laser Ablation in Analytical Chemistry. Analytical Chemistry. 85(13): 6162-6177

Russo RE, Mao XL, Liu C, Gonzalez J (2004) Laser assisted plasma spectrochemistry: laser ablation. J. Anal. At. Spectrom. 19(9): 1084-1089

Stead CV, Tomlinson EL, Kamber BS, Babechuk MG, McKenna CA (2017) Rare Earth Element Determination in Olivine by Laser Ablation-Quadrupole-ICP-MS: An Analytical Strategy and Applications. Geostand. Geoanal. Res. 41(2): 197-212

Steely AN, Hourigan JK, Juel E (2014) Discrete multi-pulse laser ablation depth profiling with a single-collector ICP-MS: sub-micron $\mathrm{U}-\mathrm{Pb}$ geochronology of zircon and the effect of radiation damage on depth-dependent fractionation. Chem. Geol. 372: 92-108

Sylvester PJ (2008) Matrix effects in laser ablation ICP-MS. Laser Ablation ICP-MS in the Earth Sciences: Current Practices and Outstanding Issues (P. Sylvester, ed.). Mineralogical Association of Canada. Short Course Series. 40: 67-78

Tabersky D, Luechinger NA, Rossier M, Reusser E, Hametner K, Aeschlimann B, Frick DA, Halim SC, Thompson J, Danyushevsky L, Gunther D (2014) Development and characterization of custom-engineered and compacted nanoparticles as calibration materials for quantification using LA-ICP-MS. J. Anal. At. Spectrom. 29(6): 955-962 
Tang M, Arevalo Jr R, Goreva Y, McDonough WF (2015) Elemental fractionation during condensation of plasma plumes generated by laser ablation: a ToF-SIMS study of condensate blankets. J. Anal. At. Spectrom. 30(11): 2316-2322

Ubide T, McKenna CA, Chew DM, Kamber BS (2015) High-resolution LA-ICP-MS trace element mapping of igneous minerals: In search of magma histories. Chem. Geol. 409: $157-168$

Wu S, Wang Y, Xu C, Yuan J (2016) Elemental Fractionation Studies of $193 \mathrm{~nm}$ ArF Excimer Laser Ablation System at High Spatial Resolution Mode. Chinese Journal of Analytical Chemistry. 44(7): 1035-1041

Wu S, Wang Y, Zhan X (2015) Research progress on reference materials for in situ elemental analysis by laser ablation-inductively coupled plasma-mass spectrometry. Rock and Mineral Analysis. 34(5): 503-511

Wu S, Wang Y, Zhan X, Kronz A, Simon K, Xu C, Tian H (2016) Study on the Elemental Fractionation Effect of CGSG Reference Materials and the Related Within-Unit Homogeneity of Major and Trace Elements. Rock and Mineral Analysis. 35(6): 612-620

Yang QC, Jochum KP, Stoll B, Weis U, Kuzmin D, Wiedenbeck M, Traub H, Andreae MO (2012) BAM-S005 Type A and B: New Silicate Reference Glasses for Microanalysis. Geostand. Geoanal. Res. 36(3): 301-313

Yang Y-H, Wu F-Y, Li Y, Yang J-H, Xie L-W, Liu Y, Zhang Y-B, Huang C (2014) In situ U-Pb dating of bastnaesite by LA-ICP-MS. J. Anal. At. Spectrom. 29(6): 1017-1023

Yuan HL, Gao S, Liu XM, Li HM, Gunther D, Wu FY (2004) Accurate U-Pb age and trace element determinations of zircon by laser ablation-inductively coupled plasma-mass spectrometry. Geostand. Geoanal. Res. 28(3): 353-370 
Zack T, Stockli DF, Luvizotto GL, Barth MG, Belousova E, Wolfe MR, Hinton RW (2011) In situ U-Pb rutile dating by LA-ICP-MS: $208 \mathrm{~Pb}$ correction and prospects for geological applications. Contributions to Mineralogy and Petrology. 162(3): 515-530

Zhang C, Hu Z, Zhang W, Liu Y, Zong K, Li M, Chen H, Hu S (2016) A green and fast laser fusion technique for bulk silicate rock analysis by laser ablation ICP-MS. Analytical Chemistry. 88(20): 10088-10094 


\section{Chapter 5 Concluding remarks and Outlook}

\subsection{Concluding remarks}

This Ph.D. project gives a detailed investigation for the further improvement of LA-ICP-MS in elemental analysis. The perspective is from laser ablation process, sample preparation technique, plasma strengthening, and data reduction protocol. The major conclusions involved in this thesis are given as follows.

- A quantitative reduction strategy consisting of Ratioing, Standardization, and Normalization (RSN) is proposed to process the LA-ICP-MS transient signal of NIST, MPI-DING, USGS and CGSG glass reference materials. The RSN strategy allows the quantitative reduction without knowing the concentration of internal standard before LA-ICP-MS analysis.

- A quantification approach based on two reference materials (NIST610 and StHs6/80-G) and bulk normalization as $100 \%$ (wt) is proposed to reduce LA-ICP-MS transient signals. This approach eliminates the deficiencies encountered with the quantification strategy using single reference material, such as the extremely low content or large uncertainty of some elements.

- Two approaches including ultrafine powder pellet and flux-free fusion glass are developed for LA-ICP-MS bulk analysis of granitic rock samples. Results illustrate that both approaches are practical for LA-ICP-MS bulk analysis of granitic samples

- Signal intensity of 54 investigated elements is enhanced up to 6 folds by using guard electrode. The addition of $\mathrm{N}_{2}$ shifts the ionization zone backward to the sample cone. The GE-on- $\mathrm{N}_{2}$ with $\left(2 \mathrm{ml} \mathrm{min}^{-1}\right)$ is the best instrument conditions for routine multiple trace element analysis 
- Mass load effect was negatively correlated with corresponding oxide melting temperature, while positively correlated with elemental $1^{\text {st }}$ ionization potential. Downhole fractionation was negligible when the ratio of ablation depth versus spot size was smaller than $1: 1$.

- The glasses and most minerals have the controllable ablation behaviors, except quartz. The ablation behaviors of powder pellets could be improved by increasing the tableting pressure or decreasing the particle grain size. Ablation rates are specified to the individual sample. Ablation rate data of 43 different sample substrates are presented.

\subsection{Outlook}

\section{What will LA-ICP-MS look like in future?}

The femtosecond laser provides a laser source with ultrashort pulse duration, which allows the ablation process with an insufficient time for photon energy to dissipate into the target lattice to heat and induce melting before the explosive release of sample material. Thus the femtosecond laser may substantially eliminate the laser-induced element fractionation (Russo et al., 2002).

Simultaneous mass analyzer detection systems (MC-ICP-MS, TOF-ICP-MS) are ideal for measuring transient laser ablation signals due to the recorded ion intensities are exactly reflected the compositional variations of the target material, which may occur more abruptly for the sequence mass analyzer (quadruple (Q)-ICP-MS and single collector ICP-MS). Recently, the excellent potential for LA-TOF-ICP-MS has been demonstrated for the capability of high-speed multi-element analysis (Gundlach-Graham and Günther, 2016).

Therefore we can imagine that the widespread adoptions of the user-friendly femtosecond laser ablation system coupled to the simultaneous mass analyzer detection systems (MC-ICP-MS and TOF-ICP -MS) in the near future. Meanwhile, the ultrafast response 
ablation cell and more powerful data reduction software are also expected.

\section{What could we do with the current and new generation of LA-ICP-MS?}

With the intensive improvement of LA-ICP-MS, we could conduct lot things either in methodology development or applications for the serving Earth science. Here I listed some of them, which is already "work in progress".

\section{Methodology:}

(1) Laser ablation split stream techniques (LISS) allows concurrent elemental and isotopic analysis of the same ablation volume, which makes the interpretation of isotopic ratios or dates in light of complementary geochemical information (Kylander-Clark et al., 2013).

(2) 2D/3D trace element mapping (Raimondo et al., 2017; Ubide et al., 2015) as a tool for visualizing and quantifying internal structure of trace element concentration in igneous minerals

(3) Nanopowder pellets technique for the enhancement the LA-ICP-MS capability in bulk rock analysis (Garbe-Schönberg and Müller, 2014) and for the development of matrix-matched reference materials (Tabersky et al., 2014).

\section{Applications:}

(1) Zircon $\mathrm{Pb}-\mathrm{U}$ and Th-U dating at a young age (10-300 ka) (Guillong et al., 2016; Guillong et al., 2014)

(2) In-situ high precision isotopic ratio analysis, for example, Li isotope composition in single crystal (e.g. zircon) for understanding the diffusion derived fractionation.

(3) High precision elemental ratio determination, especially for these elements suffered from incomplete dissolution in solution ICP-MS measurements, for example, $\mathrm{Nb} / \mathrm{Ta}, \mathrm{Zr} / \mathrm{Hf}$, etc. 


\section{Reference}

Garbe-Schönberg D, Müller S (2014) Nano-particulate pressed powder tablets for LA-ICP-MS. J. Anal. At. Spectrom. 29 (6): 990-1000

Guillong M, Sliwinski JT, Schmitt A, Forni F, Bachmann O (2016) U - Th Zircon Dating by Laser Ablation Single Collector Inductively Coupled Plasma - Mass Spectrometry (LA ICP - MS). Geostand. Geoanal. Res. 40(3): 377-387

Guillong M, von Quadt A, Shuhei S, Bachmann O, Peytcheva I (2014) LA-ICP-MS Pb-U dating of young zircons from the Kos-Nisyros volcanic centre, SE Aegean arc. J. Anal. At. Spectrom. 29(6): 963-970

Gundlach-Graham A, Günther D (2016) Toward faster and higher resolution LA-ICPMS imaging: on the co-evolution of LA cell design and ICPMS instrumentation. Anal. Bioanal. Chem. 408(11): 2687-2695

Kylander-Clark AR, Hacker BR, Cottle JM (2013) Laser-ablation split-stream ICP petrochronology. Chem. Geol. 345: 99-112

Raimondo T, Payne J, Wade B, Lanari P, Clark C, Hand M (2017) Trace element mapping by LA-ICP-MS: assessing geochemical mobility in garnet. Contributions to Mineralogy and Petrology. 172(4): DOI 10.1007/s00410-00017-01339-z

Russo RE, Mao XL, Liu HC, Gonzalez J, Mao SS (2002) Laser ablation in analytical chemistry - a review. Talanta. 57(3): 425-451

Tabersky D, Luechinger NA, Rossier M, Reusser E, Hametner K, Aeschlimann B, Frick DA, Halim SC, Thompson J, Danyushevsky L, Gunther D (2014) Development and 
characterization of custom-engineered and compacted nanoparticles as calibration materials for quantification using LA-ICP-MS. J. Anal. At. Spectrom. 29(6): 955-962

Ubide T, McKenna CA, Chew DM, Kamber BS (2015) High-resolution LA-ICP-MS trace element mapping of igneous minerals: In search of magma histories. Chem. Geol. 409: 157-168 


\section{Appendix A: Curriculum vitae}

\section{Shitou Wu}

Date of birth: 28.09.1987

Place of birth: Hebei (China)

\section{Scientific education:}

07/2011 Bachelor of sciences (Analytical chemistry)

Thesis: Structure and Corrosion Resistance of $\gamma$-APS

( $\gamma$-Aminopropyltriethoxysilane) Silane Film on Low Carbon Steel

07/2013 Master of sciences (Environmental chemistry)

Thesis: The Evaluation of Rare Earth Elements Analytical Methods in Bayun Obo Rare Earth Ore Reference Material Candidates and A Preliminary Study on The Rare Earth Elements Speciation

10/2013-08/2017: Doctorate at the Geoscience Center Göttingen (Geochemistry) Thesis: Laser Ablation-Inductively Coupled Plasma-Mass Spectrometer (LA-ICP-MS) in Geosciences: Further Improvement for Elemental Analysis (supervisor: Prof Gerhard Wörner and Dr. Klaus Simon)

\section{Work experience:}

2013-2017: LA-ICP-MS laboratory assistant

\section{Field experience:}

$\begin{array}{llll}2014 & \text { Germany } & 2 \text { days } & \text { Regionale Geologie der Umgebung von Göttingen (Nordhessen) } \\ 2015 & \text { Germany } & 2 \text { days } & \text { Magmatite und Metamorphite (Harz) } \\ 2015 & \text { Germany } & 2 \text { days } & \text { Sedimentgesteine und Fazies } \\ 2016 & \text { Germany } & 1 \text { week } & \text { Vulkanfeldkurs (Laacher See) }\end{array}$




\section{Short course:}

08/2015 Application of diffusion studies to the determination of timescales in geochemistry and petrology. Bochum

11/2015 Introduction to Secondary Ion Mass Spectrometry in the Earth Sciences. Potsdam

02/2017 MATLAB Recipes for Earth Sciences. Potsdam

03/2017 Radiogenic and Non-tradional Isotope short course, Frankfurt 


\section{Appendix B: Publications}

\section{Manuscripts and Articles*}

- Shitou $\boldsymbol{W u}^{*}$ and Klaus Simon. (2017) Signal enhancement in LA-ICP-MS analysis by guard electrode and the addition of nitrogen and hydrogen into carrier gas flow: A perspective from experiment (in preparation for "Journal of Analytical Atomic Spectrometry")

- Shitou Wu*, Volker Karius, Burkhard C Schmidt, Klaus Simon and Gerhard Wörner. (2017) Comparison of ultrafine powder pellet and flux-free fusion glass for bulk analysis of granitic rock samples by Laser Ablation-Inductively Coupled Plasma-Mass Spectrometry. ("Geostandards and Geoanalytical Research", under review)

- Shitou $\boldsymbol{W u}^{*}$ and Klaus Simon. (2017) LA-ICP-MS transient signal quantification of NIST, MPI-DING, USGS, and CGSG glass reference materials by Ratioing, Standardization, and Normalization (RSN) ("Geostandards and Geoanalytical Research”, under review).

- Shitou Wu, Yaping Wang*, and Chunxue Xu. (2017) Laser ablation-inductively coupled plasma-mass spectrometer: A quantification strategy based on two reference materials and bulk normalization as 100\% (wt). Chinese Journal of Analytical Chemistry. 45(7): 965-972.

- Shitou Wu, Chunxue Xu, Klaus Simon, Yilin Xiao and Yaping Wang. (2017) Ablation behaviors of $193 \mathrm{~nm}$ ArF excimer laser system for the selected substrates. Rock and Mineral Analysis. (Accepted, in Chinese with English abstract).

- Shitou Wu, Yaping Wang*, Chunxue Xu and Jihai Yuan. (2016) Elemental fractionation studies of $193 \mathrm{~nm}$ ArF excimer laser ablation system at high spatial resolution. Chinese Journal of Analytical Chemistry. 44(7): 1035-1041. 
- Shitou Wu, Yaping Wang*, Xiuchun Zhan, Andreas Kronz, Klaus Simon, Chunxue Xu and Huan Tian. (2016) Study on the elemental fractionation effect of CGSG reference materials and the related within-unit homogeneity of major and trace elements. Rock and Mineral Analysis. 35(6):612-620 (in Chinese with English abstract)

- Shitou, Yaping Wang*, Chunxue Xu (2015) Research progress on reference materials for in situ elemental analysis by laser ablation-inductively coupled plasma-mass spectrometry. Rock and Mineral Analysis. 34(5): 503-511. (in Chinese with English abstract)

- Shitou Wu, Yaping Wang*, Dezhong Sun, Hongli Wen, Chunxue Xu and Wei Wang (2014) Determination of 15 rare earth elements in rare earth ore by inductively coupled plasma-atomic emission spectrometry: A comparison of four different pretreatment methods. Rock and Mineral Analysis. 33(1) 12-19. (in Chinese with English abstract)

\section{Conference abstracts*}

- Shitou Wu, Volker Karius and Gerhard Wörner (2017) Laser ablation ICP-MS combination with nano-powder pellets and applications to granite bulk rock analysis. EGU Conference, Vienna. (Poster)

- Shitou Wu, Klaus Simon and Gerhard Wörner (2016) Matrix effect studies of thirteen international reference materials based on 193nm ArF LA-SF-ICP-MS. European workshop on laser ablation, Ljubljana. (Poster)

- Shitou Wu, Klaus Simon, Burkhard C Schmidt and Gerhard Wörner (2015) In situ analysis of major and trace elements of hydrous silicate glasses and minerals by laser ICPMS: the effect of water. Goldschmidt Conference, Prague. (Poster) 
- Shitou $\boldsymbol{W u}$ and Klaus Simon (2015) Evaluation Study of NIST612 Transient Data obtained in line scan and discrete spot modes by LA-SF-ICP-MS. European Winter Conference on Plasma Spectrochemistry. Münster. (Poster)

- Shitou Wu, Klaus Simon and Gerhard Wörner (2014) Rapid and Precise Determination of Major and Trace Elements in Milk in Pressed Powder Pellet Targets by Laser Ablation ICP-MS: Cs as Internal Standard and Matrix Matched Calibration. European workshop on laser ablation, London. (Poster) 


\section{Appendix C: Supplementary materials}

Supplementary material is provided in digital form. The attached DVD contains:

Appendix C_Chapter2.1.rar

Appendix C_Chapter3.1.rar 
Appendix C: Supplementary materials 